\title{
Microstructural and chemical characterization of medium burnup fast reactor MOX using electron microscopy
}

\section{February 2021}

Fabiola Cappia, Jason Harp

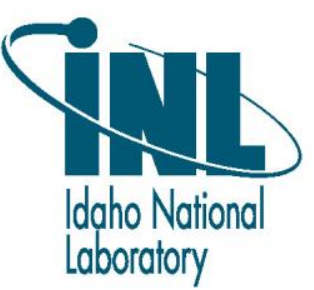




\section{DISCLAIMER}

Neither the U.S. Government nor any agency thereof, nor any of their employees, makes any warranty, expressed or implied, or assumes any

legal liability or responsibility for the accuracy, completeness, or usefulness, of any information, apparatus, product, or process disclosed, or represents that its use would not infringe privately owned rights.

References herein to any specific commercial product, process, or service by trade name, trade mark, manufacturer, or otherwise, does not necessarily constitute or imply its endorsement, recommendation, or favoring by the U.S. Government or any agency thereof. The views and opinions of authors expressed herein do not necessarily state or reflect those of the U.S. Government or any agency thereof. Being provided this document, directly or indirectly, shall not be construed to constitute a governmental export license or authorization. 


\section{Microstructural and chemical characterization of medium burnup fast reactor MOX using electron microscopy}

Fabiola Cappia, Jason Harp

Idaho National Laboratory

Characterization and Advanced PIE Division

Idaho Falls, Idaho 83415

http://www.inl.gov

Prepared for the

U.S. Department of Energy

Office of Nuclear Energy

Under DOE Idaho Operations Office

Contract DE-AC07-05ID14517 
Page intentionally left blank 


\section{ACKNOWLEDGEMENTS}

The authors would like to thank A. Winston and J.F. Jue for collecting the SEM data. We are also indebted to F. Teng and D. Murray who performed the TEM sample preparation and EBSD data collection, and to B. Miller, B. Koombaiah and L. He who collected the TEM data. K. Wright is acknowledged for performing the EPMA measurements. We would also like to thank C. Knight, the EML and IMCL operation and radioprotection staff for technical and administrative support to this project. 
Page intentionally left blank 


\section{CONTENTS}

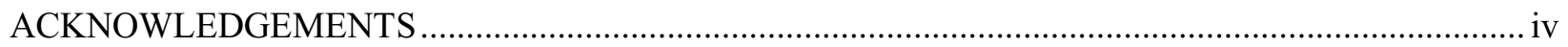

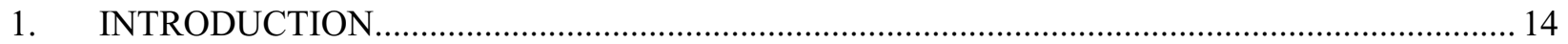

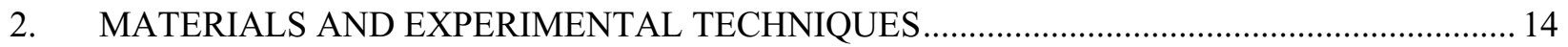

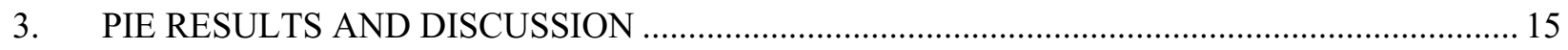

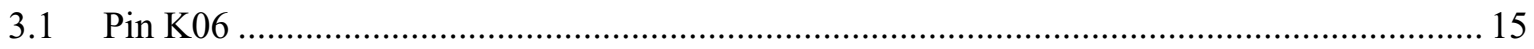

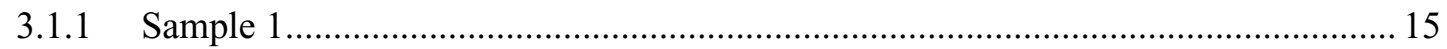

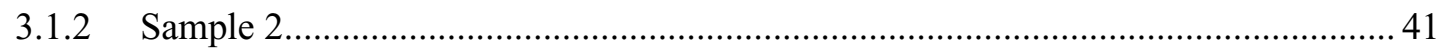

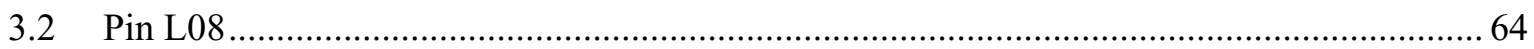

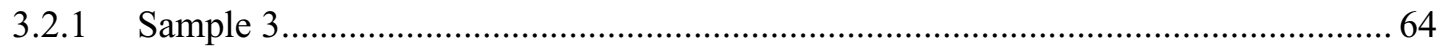

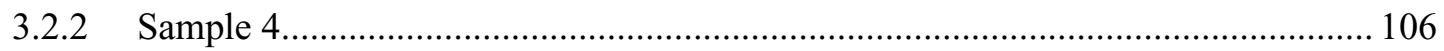

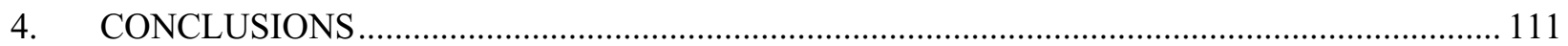

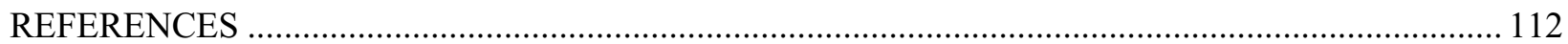

\section{FIGURES}

Figure 1. (a) Overview of sample 1. (b) Low magnification SEM image. .............................................. 16

Figure 2. Five metal precipitates approximate composition as a function of the pellet radius.................. 17

Figure 3. Large inclusion observed between the equiaxed and columnar grain region........................... 18

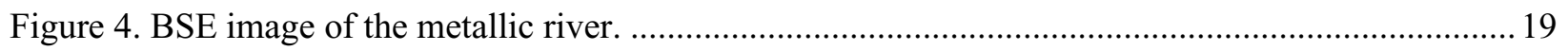

Figure 5. Bright-field TEM image of the structure at the interface between the metallic river and

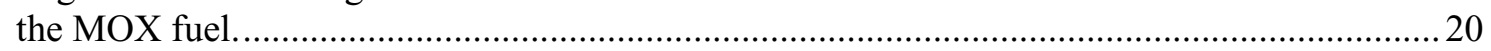

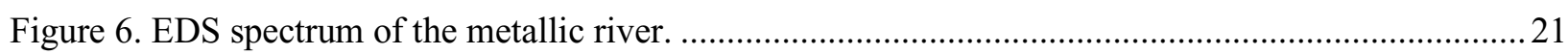

Figure 7. EDS maps (in at \%) sowing the presence of fission gas bubbles in the Pd matrix.................... 22

Figure 8. EDS spectrum of the fission product deposit shown in Figure 4............................................. 23

Figure 9. (a) High magnification of the outermost part of the fuel pellet at the end of the metallic river. (b) Qualitative map of Cs signal. (c) Comparison of the spectra from the boxes in a showing the enrichment of the alkali metals with respect to the matrix

Figure 10. (a) BSE of another peripheral location adjacent to the metallic river. (b) Cs qualitative map. (c) Overlay of the EDS signal from Ag (red) and the WDS signal of Pd (blue).

The purple color is the result of the overlap.

Figure 11. Inverse Pole Figure (IPF) overlaid to the image quality (IQ) map of the area at the interface between the fission product rich zone and the fuel matrix.

Figure 12. SEM image showing a "cauliflower-type" structure at the outermost fuel periphery.

Figure 13. Inverse Pole Figure (IPF) overlaid to the image quality (IQ) map of the area outside the "cauliflower" shown in the previous figure.

Figure 14. BSE image of the fuel to cladding region from area 2. 
Figure 15. SE image of the fuel to cladding region from area 3.

Figure 16. (a) BSE image of the fuel to cladding area from area 4. (b)-(e) EDS maps of fuel major component and fission product. The rectangle in (a) indicates where the TEM lamella was extracted.

Figure 17. Overview of intergranular corrosion in the HT-9 cladding. .

Figure 18. Other location in the HT-9 cladding with initial stages of corrosion. Cs and Te are associated with the corroded area. 30

Figure 19. Low magnification bright field images of the lamellae. .33

Figure 20. High magnification images corresponding to (a) region 1 and (b) region 2.......................... 35

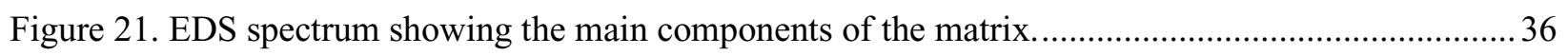

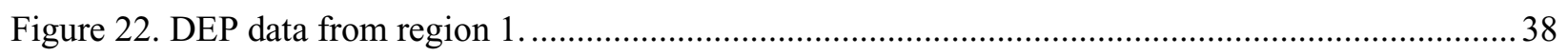

Figure 23. (a) Representative diffraction rings pattern from the nanocrystalline area of the lamella. (b) Isolated large grain present in the lamella.

Figure 24. (a) Dark Field STEM image of the nanocrystalline domain. (b) EDS map showing Cs enrichment in some of the grains (map shows at $\%$ ). .39

Figure 25. Atomic map of Te corresponding to the lamella area shown in Figure 19b...... .39

Figure 26. Atomic maps of the lamella from area 4.

Figure 27. Spectra corresponding to area 1 and 2 in Figure 26a. The different enrichment in $U$ and $\mathrm{Pu}$ can be appreciated by the relative height of the characteristic main peak.

Figure 28. Overview of sample 2

Figure 29. (a) BSE image of the grains close to the central hole. (b) High magnification of one of the grains showing the grain surface decorated with gas bubbles and metallic precipitates.

Figure 30. Accumulation of grey phases along a large crack surface.

Figure 31. (a) Region showing unconventional grain morphology within the equiaxed grain area.

(b) Spectra corresponding to the marked regions in (a)

Figure 32. IPF and IQ map outside the restructured pool from Figure 31a..... .45

Figure 33. (a)-(b) Examples of hole surface showing grain subdivision.

Figure 34. Missing fuel piece from area 2 showing onset of grain subdivision on the surface of pre-existing grains.

Figure 35. Onset of intergranular corrosion at the inner surface of the cladding surface.

Figure 36. BSE image showing the Pd liner on the inner cladding surface.

Figure 37. (a) BSE image of FCCI. (b)-(h) Chemical mapping of fission products and major cladding components. No $\mathrm{U}$ and $\mathrm{Pu}$ were present in the corrosion island.

Figure 38. (a) BSE image of FCCI. (b)-(f) Chemical mapping of fuel, fission products and major cladding components. Maps in (c) and (e) correspond to WDS signal, whereas the others are EDS maps. 
Figure 39. (a) BSE image of FCCI. (b)-(h) Chemical mapping of fuel, fission products and major cladding components. Maps in (e) and (g) correspond to WDS signal, whereas the others are EDS maps....

Figure 40. (a) Region with increased FCCI thickness, showing existence of four regions with different compositions showed in (b).

Figure 41. Overview of the lamella extracted between zone 2 and 3 in Figure 40a. The red circle indicates the location of the SAED patterns in Figure 43.

Figure 42. (a) HAADF of the interface between the HT-9 and the Cr-rich region. (b), (c) Qualitative EDS maps showing precipitation of $\mathrm{Pd}$ along grain boundaries and the $\mathrm{Cr}$ rich interface. (d) Normalized element concentration across the two phases.

Figure 43. (a), (b) (a), (b) SAED patterns from the Cr-rich phase fitted using the tetragonal structure of $\sigma-\mathrm{FeCr}$. The location of the SAED patterns is shown in Figure 40.

Figure 44. HAADF image of the (a) upper part of the lamella, (c) lower part of the lamella. (b), (d) EDS qualitative maps of the most prominent fission products in the two parts, respectively. The inset shows the nanocrystalline structure of the Te and I-rich region............56

Figure 45. EDS spectrum of the nanocrystalline precipitate in the red circle in Figure 44a.

Figure 46. Comparison of the sample local conditions with the schematic of the primary environmental domains for the cladding interaction mechanisms.

Figure 47. (a) High magnification of the fuel extreme periphery from Figure 36. (b) EDS spectra corresponding to the areas marked by the red rectangles in (a).

Figure 48. Overview of the lamella from Figure 47.

Figure 49. (a) HAADF image of the fission product precipitates in between the Cr-Fe areas. (b)-

(f) Atomic maps of the major components. (g) Line scan from (a).

Figure 50. (a) HAADF from a second location containing the fission products. (b)-(c) Atomic maps of major components. (d) SAED pattern corresponding to the Cs-rich area.

Figure 51. Optical microscopy overview of sample 3.

Figure 52. Micrographs showing fission products inclusions at different radial locations.

Figure 53. Spectra comparison between the FMP and the Mo and Cs- bearing precipitate at the pellet periphery from Figure 52c. .66

Figure 54. Additional compositional measurements of the FMP measured with EPMA.

Figure 55, BSE image of the large metallic inclusions from area 1 in Figure 51

Figure 57. (a) BSE overview and (b)-(h) chemical maps from EPMA over the area showing extensive fission product precipitation at the fuel periphery.

Figure 58. HAADF image of the lamella, showing the various phases. The points indicate the location of the EDS spectra shown in the next figures.

Figure 59. (a) EDS spectrum 1 from the metallic phase region. (b) Pseudo-ternary phase diagram of $\mathrm{Mo}(\mathrm{Pd}, \mathrm{Rh})(\mathrm{Ru}, \mathrm{Tc})$, modified from Ref. [6]. The red point represents the composition of the phase measured from the EDS spectrum in (a). (c) SAED pattern indexed according to the hexagonal phase predicted in the phase diagram. 74

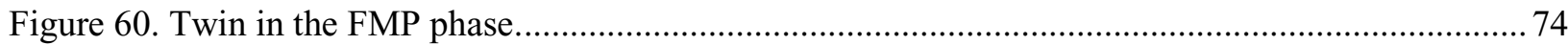

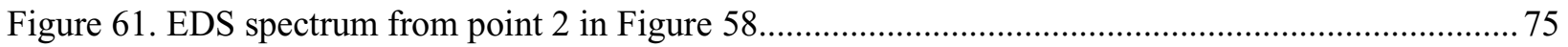


Figure 62. (a) Large grey phase inclusion at the interface between the MOX matrix and the FMP.

(b) corresponding EDS spectrum

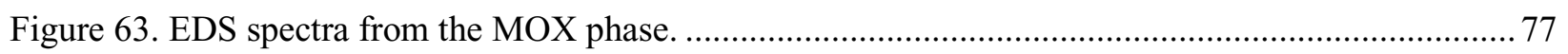

Figure 64. Images of the extended defects in the MOX phase. (a) dislocation loops, (b)

dislocation lines pinned around the grey phase at the interface with the metallic phase............ 78

Figure 65. FCCI region. (a) BSE image, (b)-(e) most significant fission products, (f) U signal................ 80

Figure 66. FCCI region. (a) BSE image, (b)-(d) most significant fission products................................. 81

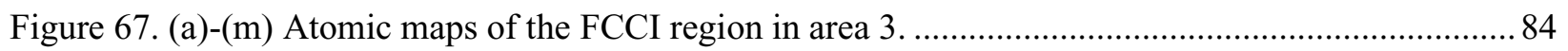

Figure 68. (a) BSE image of the FCCI from area 3 in Figure 51. (b)-(m) Atomic maps obtained with the EPMA.

Figure 69. Higher magnification image of a thick portion of the FCCI in area 3.................................. 88

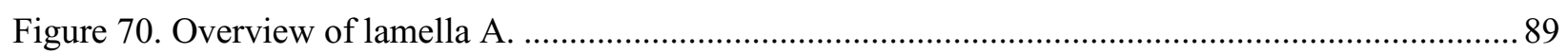

Figure 71. Enlarged view of the upper part of the lamella, highlighting the nano crystallites (white agglomerates) embedded in the epoxy matrix (mid-grey matrix).

Figure 72. Distribution of the lamella's main components. (a) HAADF image, (b) EDS maps showing the at $\%$ of $\mathrm{Cs}, \mathrm{Ba}$ and $\mathrm{Te}$. (c) Te and $\mathrm{O}$.

Figure 73. Typical morphology and diffraction pattern collected from the Te-rich phase.

Figure 74. (a) Te-rich particle composed of two crystalline domains. (b) SAED pattern indexed by the pure Te structure. (c) SAED pattern indexed with the CsTe4 structure.

Figure 75. (a) Ba-rich region probed for crystallographic information. (b) Corresponding ring pattern identified with a Cs2Ba2O3-type of structure.

Figure 76. Overview of lamella B. (a) Side close to lamella A. (b) side capturing the interface with the cladding.

Figure 77. (a) HAADF image of the chemical mapping of the side of the lamella closes to the previous. (b) O qualitative map.

Figure 78. (a) Area closest to the cladding. (b) line scan of the major components. 101

Figure 79. (a) Radial quantitative profile of (a) $\mathrm{U}$ and $\mathrm{Pu}$, showing actinide thermomigration. (b) Am and $\mathrm{Np}$ 103

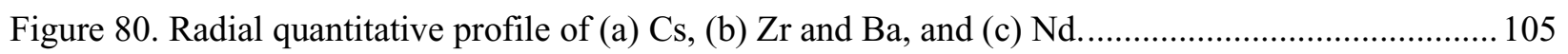

Figure 81. Radial burnup profile derived from $\mathrm{Nd}$ measurements. ....................................................... 105

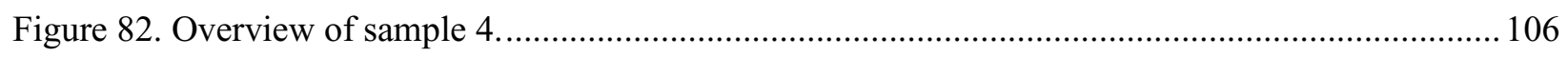

Figure 83. Fresh fracture image of the columnar grains close to the central hole. ................................ 107

Figure 84. Grey phases observed between the columnar and equiaxed grain region. ............................ 107

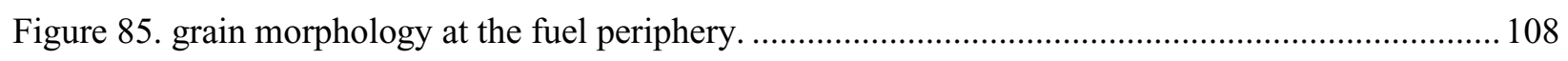

Figure 86. Other location where grain subdivision was observed. On the right side of the image, it can be seen that part of the fuel has remained attached to the FCCI.

Figure 87 . Region where a thick part of the fuel remained attached to the cladding. 


\section{TABLES}

Table 1: Summary of the main characteristics of the samples under analysis........................................ 14

Table 2. Composition of the precipitate circled in Figure 44a..............................................................5

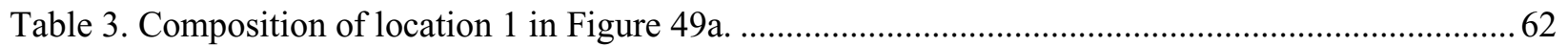

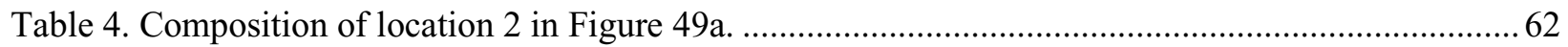

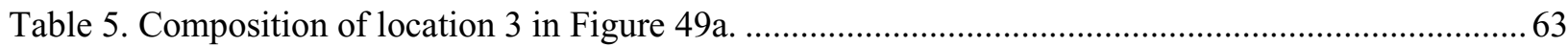

Table 6. Chemical composition of the fission product precipitates at different radial locations. ............... 66

Table 7. Uranium and plutonium quantification in the different grains of the MOX phase..................... 77

Table 8. Main components in the various phases in Figure 72 ....................................................... 92

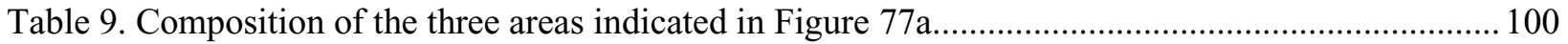

Table 10. Semi-quantitative composition of the grey phases measured by EDS.................................. 108 
Page intentionally left blank 


\section{ACRONYMS}

BSE Backscattered electron

DEP Diffraction Electron Precession

DOE Department of Energy

EBSD Electron backscatter diffraction

EDS Energy dispersive spectroscopy

EML Electron Microscopy Laboratory

EPMA Electron Probe Micro Analyzer

FCCI Fuel-cladding chemical interaction

FIB Focused-Ion Beam

FMP Five metal precipitates

GOS Grain Orientation Spread

HAADF High-Angle Annular Dark Field

HBS high burnup structure

IMCL Irradiated Materials Characterization Laboratory

INL Idaho National Laboratory

IPF Inverse Pole Figure

IQ Image quality

LMFBR Liquid Metal Fast Breeder Reactor

LWR Light Water Reactor

MOX Mixed Oxide

PIE Post-Irradiation Examination

PWR Pressurized Water Reactor

SAED Selected Area Electron Diffraction

SE Secondary Electron

SEM scanning electron microscopy

SFR Sodium Fast Reactor

STEM Scanning Transmission Electron Microscopy

TEM Transmission Electron Microscopy

WDS Wavelength dispersive spectroscopy 
Page intentionally left blank 


\section{Microstructural and chemical characterization of medium burnup fast reactor MOX using electron microscopy}

\section{INTRODUCTION}

A thorough post-irradiation examination campaign of medium burnup Sodium Fast Reactor (SFR) mixed oxide (MOX) fuel has been undertaken over the past two years to better understand the performance at intermediate burnup of this annular fuel design. Non-destructive examinations as well as destructive examinations of two pins have been recently published by the authors [1], providing data regarding the performance at macro-scale. The analyses of selected samples have continued to provide more details regarding the overall microstructure and, particularly, the fuel-cladding interaction.

\section{MATERIALS AND EXPERIMENTAL TECHNIQUES}

Fuel microstructural characterization via scanning electron microscopy/transmission electron microscopy (SEM/TEM) has been recently published as part of other projects $[2,3]$. These investigations were focused on the chemistry of fission products inside the fuel and defect analysis. This work was aimed primarily at the examination of the fuel-cladding chemical interaction (FCCI) region, but data regarding the chemical composition of fission product precipitates have also been evaluated, particularly for large agglomerations of secondary phases when encountered.

Two samples from each pin cut for destructive examinations were selected to undergo SEM and TEM examinations. The characteristics of each sample are summarized in Table 1. In addition, for the sample with highest burnup, SEM analyses were complemented by Electron Probe Micro Analyzer (EPMA) to study the radial migration of actinides and provide a more accurate analysis of the chemical composition of the secondary phases.

Table 1: Summary of the main characteristics of the samples under analysis.

\begin{tabular}{ccccc}
\hline Pin ID & Sample ID & $\begin{array}{c}\text { Relative axial } \\
\text { location (/) }\end{array}$ & Local burnup (\% FIMA) & $\begin{array}{c}\text { Expected cladding } \\
\left.\text { temperature range ( }{ }^{\circ} \mathbf{C}\right)\end{array}$ \\
\hline K06 & 1 & 0.35 & 5.15 & $409-427$ \\
& 2 & 0.75 & 4.36 & $488-508$ \\
L08 & 3 & 0.35 & 6.32 & $491-513$ \\
& 4 & 0.75 & 5.00 & $586-610$ \\
\hline
\end{tabular}

Both Secondary Electron (SE) and Backscattered Electron (BSE) micrographs were collected using a JEOL JSM-7000F field emission gun SEM equipped with wavelength dispersive spectroscopy (WDS) and energy dispersive spectroscopy (EDS) Oxford INCA software. EBSD analyses were performed using the EDAX TEAMS software, while the data have been analyzed using the OIM v.8 software from EDAX. TEM lamellae were extracted from the samples using a FEI Ga Quanta 3D FEG dual-beam FIB system. They were created by coarse trenching $10 \mu \mathrm{m}$ × $2 \mu \mathrm{m}$ x $10 \mu \mathrm{m}$ samples. Sample thinning to TEM transparency was performed in steps following different combinations of voltage and current, specifically: $30 \mathrm{kV} / 3 \mathrm{nA}-30 \mathrm{kV} / 1 \mathrm{nA}-30 \mathrm{kV} / 0.5 \mathrm{nA}$ till final cleaning with $8 \mathrm{kV} / 77 \mathrm{pA}-5 \mathrm{kV} / 48 \mathrm{pA}$. Both a FEI Titan Themis $200 \mathrm{X}$-FEG TEM equipped with a Super-X EDS system and a FEI Talos were used for semiquantitative chemical analysis of the lamellae. Selected Area Electron Diffraction (SAED) data were collected to perform crystallographic analyses. The indexing of the SAED patterns was performed using the Crystallographic Toolbox CrysTBoX [4]. An algorithm using the built-in Matlab 2019b ${ }^{\circledR}$ nonlinear least-squares data-fitting solver was developed to obtain the set of lattice constants from the experimentally measured d-spacing and plane indexes calculated with CrysTBoX. Electron probe 
microanalysis was performed using a Cameca SX100R electron probe microanalyzer, which is specifically designed for the analysis of highly radioactive specimens.

Various column conditions were employed depending on the phase type and element concentration. In all cases, the accelerating voltage was $25 \mathrm{kV}$. For quantitative traverse, the beam size was just focused. For FCCI analyses, the beam was defocused to $5 \mu \mathrm{m}$. For quantitative radial traverses, the current used was $200 \mathrm{nA}$, whereas, for precipitates and cladding constituents, the current varied from $20 \mathrm{nA}$ to $200 \mathrm{nA}$ depending on the number of counts generated. X-ray maps were collected with a current of $50 \mathrm{nA}$.

\section{PIE RESULTS AND DISCUSSION}

\subsection{Pin K06}

\subsubsection{Sample 1}

\subsubsection{Fuel microstructure and fission products}

The overview of the first sample taken with the optical microscope is given in Figure 1a. Low-magnification images have been acquired using the SEM (Figure 1b) and confirm the presence of an equiaxed grain region and a columnar grain region. Manual measurements of the extension of the columnar grain area have been made. Based on the average measurement made on SEM images, the columnar grain region extends through $0.66 \pm 0.01(/)$ of the fuel radius, in relative units. The value is slightly below the one calculated from texture analysis with the optical microscopy, where a value of 0.71 for the columnar grain extension was obtained. As the resolution of the optical microscope was limited and the samples were not etched, some discrepancy between the two methods is reasonable.

(a)

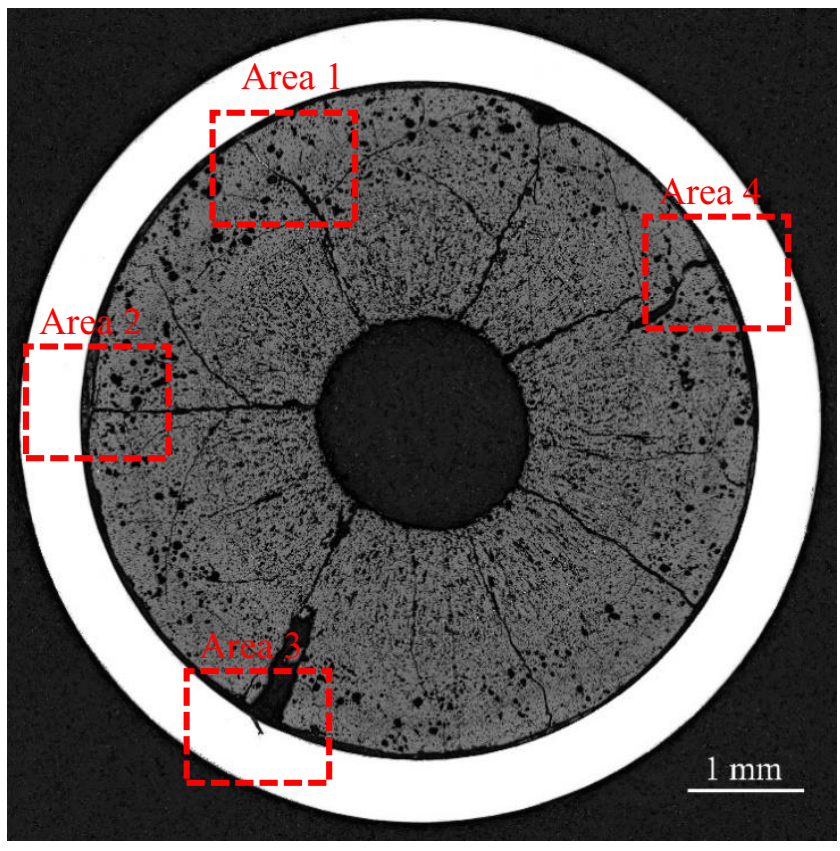




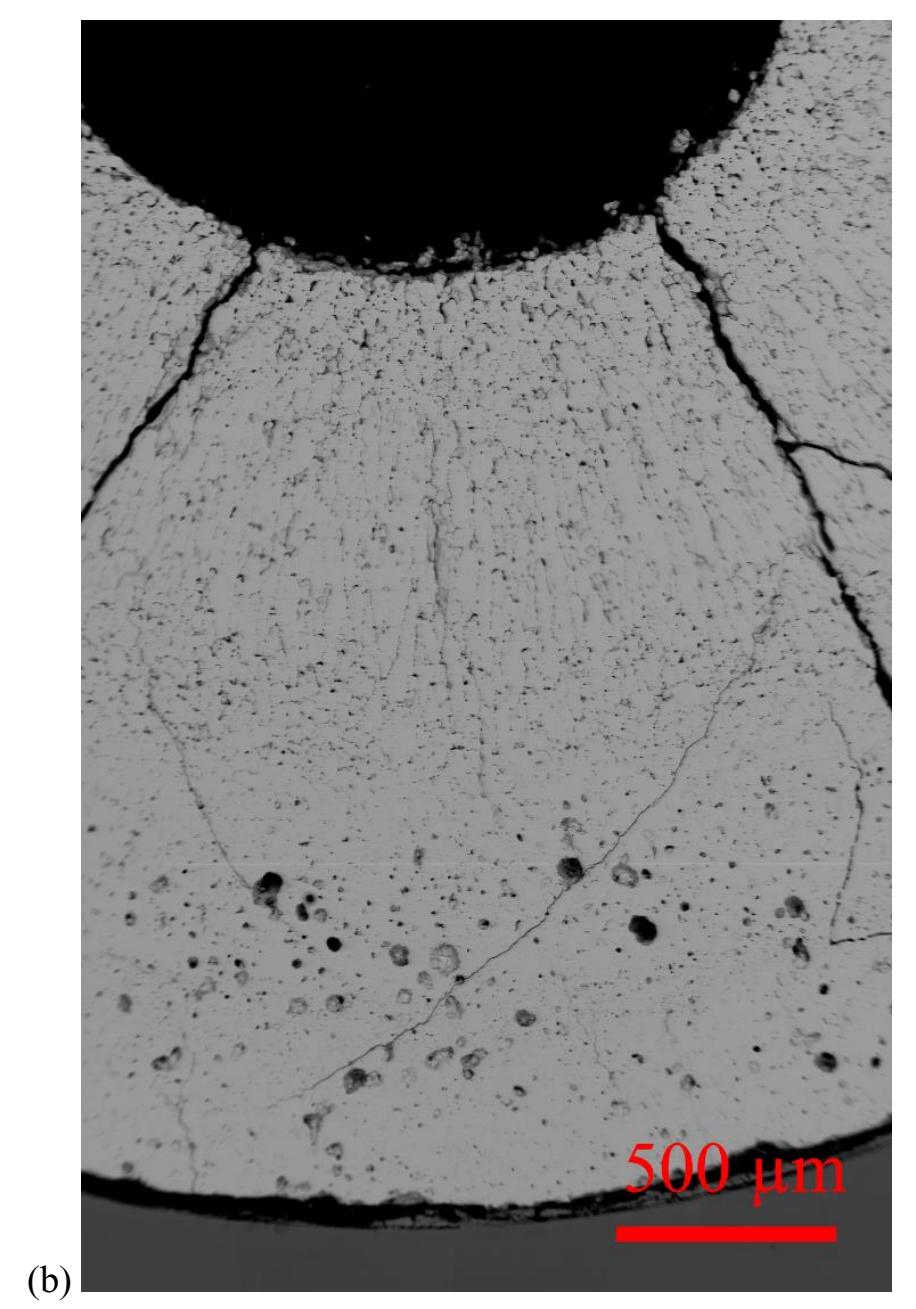

Figure 1. (a) Overview of sample 1. (b) Low magnification SEM image.

The presence of fission product precipitates was already clear from the optical microscopy analyses previously performed on these samples. Semi-quantitative EDS analyses have been done at different radial locations to map the evolution of the metallic precipitates. The radial measurements are shown in Figure 2. The increase of $\mathrm{Pd}$ concentration towards the fuel periphery is a consequence of its higher volatility compared to the other products. Of particular interest is the ratio between $\mathrm{Mo}$ and $\mathrm{Ru}$, as the decrease in Mo concentration in the metallic precipitates is used to monitor the oxygen potential of the fuel $[5,6]$. It can be seen that the ratio between Mo and Ru remains approximately constant and close to one, a sign that oxidation of Mo has not occurred to significant extent in the majority of the pellet radius. Precipitates in the outermost part of the pellet, i.e., for $r / r_{0}>0.8$ were too small to be seen and measured via SEM/EDS and were analyzed using TEM instead. 


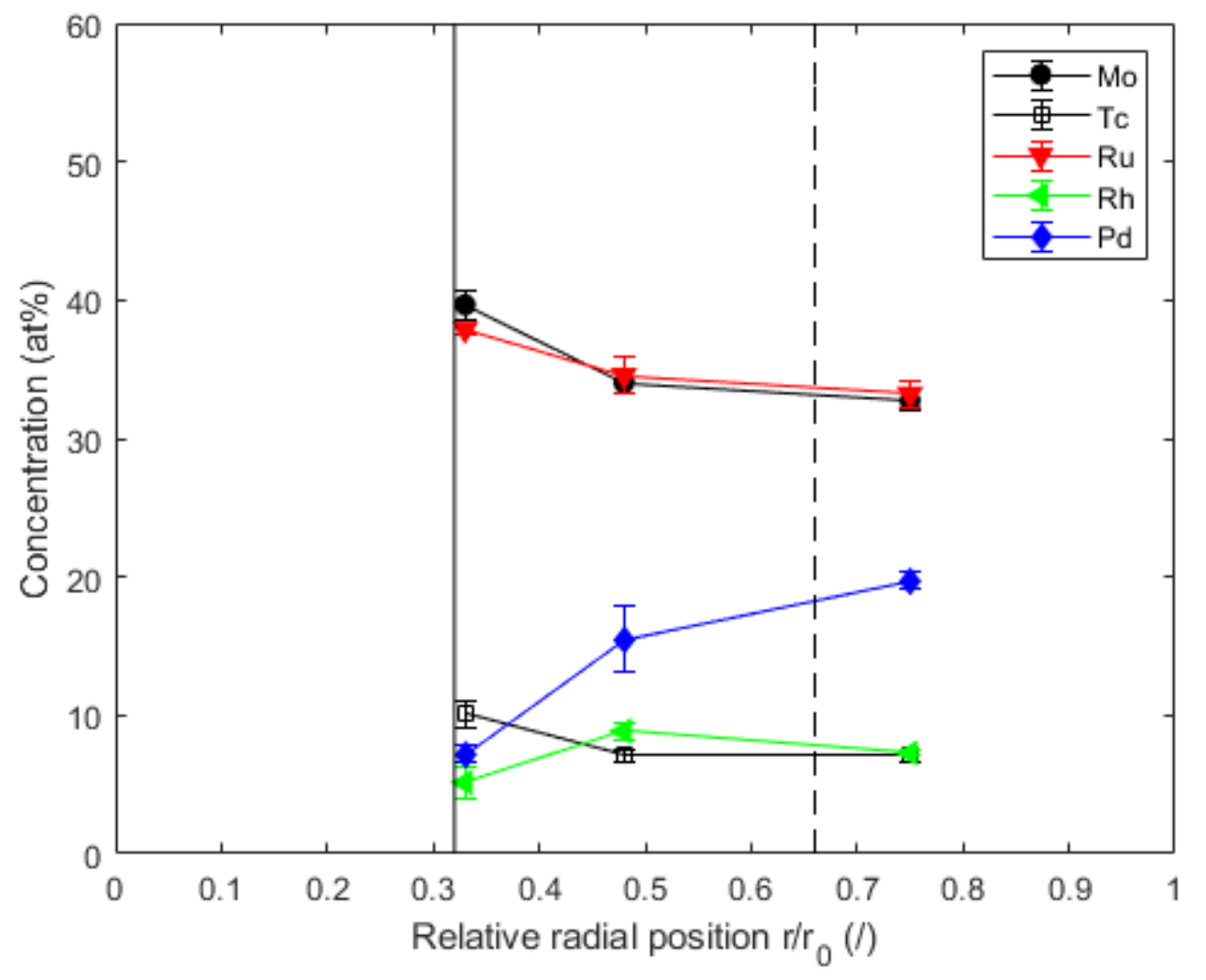

Figure 2. Five metal precipitates approximate composition as a function of the pellet radius. The black solid line represents the central hole, while the dashed line is the extension of the columnar grain as measured manually from SEM images.

Close to the border between the columnar grain region and the equiaxed one, a precipitate with both a metallic phase and an oxide phase was observed (Figure 3).

Co-existence of grey phases and metallic precipitates in large inclusions towards the periphery of the fuel has been already reported by Sato et al. [7]. A possible explanation is a multiple stage condensation of the insoluble fission products on the surface of a large pore. It is likely that the grey phases precipitated at an early stage, followed by later precipitation of the metallic fission products. 


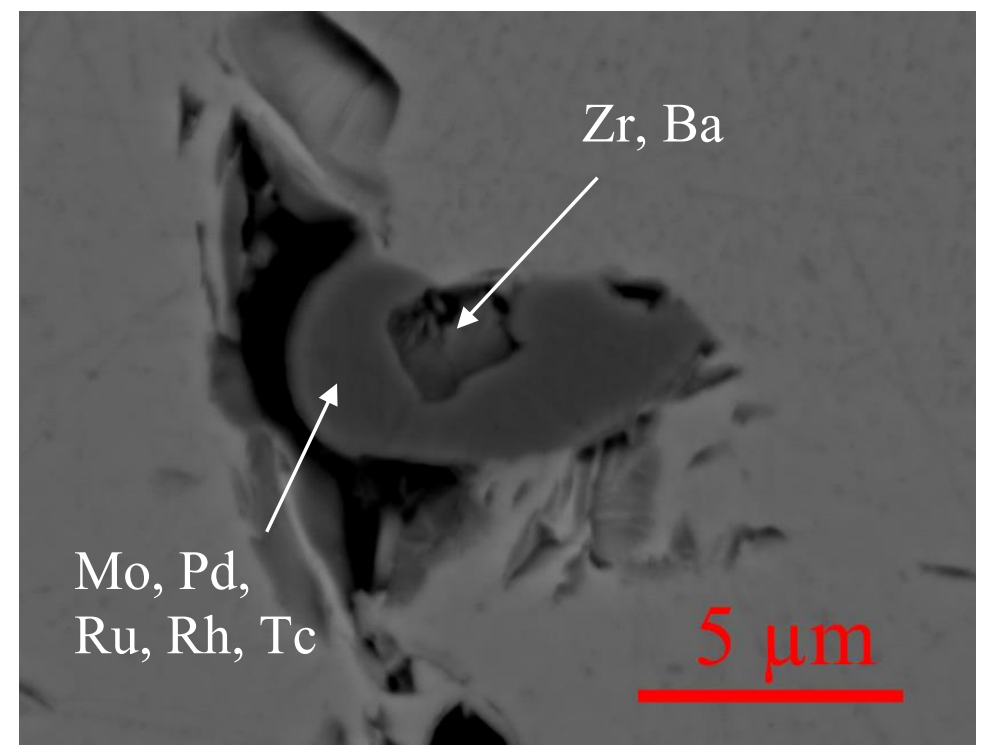

Figure 3. Large inclusion observed between the equiaxed and columnar grain region. The inclusion is formed by a core of oxide grey phase and a metallic shell.

In the equiaxed grain region indicated by the square Area 1 in Figure 1a, a "metallic river" was observed, which is shown in Figure 4. Metallic rivers were associated with transport of cladding components, particularly Fe, into the fuel [8]. Qualitative EDS spectra of the river indicated that, in this case, the main component is Pd. More in-depth analyses of the river have been performed with TEM, which are shown in Figure 5 through Figure 7. In Figure 5a, an overview of the interface between the fuel and the metallic river is presented. On one side, the metallic phase exhibits large grains with presence of twinning. In the lower part of the lamella, the fuel shows a crystalline structure with sub-micrometric grains close to the interface. The diffraction patterns for the two phases are shown in Figure $5 \mathrm{~b}$ and $\mathrm{c}$. Chemical analysis of the metallic phase by TEM confirmed the SEM results, showing that the phase is almost entirely composed of Pd (see Figure 6), with minor content of Mo. Traces of U and Pu are also detected, but they could be due to the interference of signal from the bulk of the lamella. The diffraction pattern in Figure $5 \mathrm{~b}$ is in excellent agreement with the fcc structure of pure Pd (space group Fm $\overline{3} \mathrm{~m}$, $\mathrm{n} 225$ ). The derived lattice parameter is $3.86 \AA \pm 0.01 \AA$ from this diffraction pattern. A slightly larger value of $3.88 \AA \pm 0.01 \AA$ was obtained from a second data set. Both data sets are comparable with literature values $[9,10]$. Twin grains are present, as indicated in Figure $5 \mathrm{~b}$. The full description of the twinning of the Pd structure is beyond the scope of these analyses, butformation of twins, which are generally a consequence of deformation or annealing growth, is not totally surprising. The metallic river has likely formed over the in-reactor life, and the area experienced steep temperature gradients which, in turn, translate in stress gradients. Under such circumstances, twinning of an fcc structure could have been caused.

Even at low magnification, bubbles can be seen in the Pd phase in Figure 5. A high magnification image of the bubbles as well as the atomic concentrations of Xe and Cs are shown in Figure $7 \mathrm{a}, \mathrm{b}$ and $\mathrm{c}$, respectively. Xe has been injected into the metallic phase via fissioning processes and have formed bubbles within the metal. The precipitation of Xe indicates insolubility of the gas in the Pd, in line with atomistic calculations of solution and accommodation energies of this product in $\varepsilon$ phases [11]. It is interesting to note the precipitation of Cs on the surface of the bubbles in Figure 7c. Cs is also predicted to be insoluble in epsilon phases [11], Cs could both be a decay product of Xe-133, but signature of Ba within the bubbles was also measured, indicating that part of the Cs could be Cs-137 or Cs-134. To the author's knowledge, this is the first time the co-existence of gaseous and volatile fission products is directly reported in the metallic precipitates. 


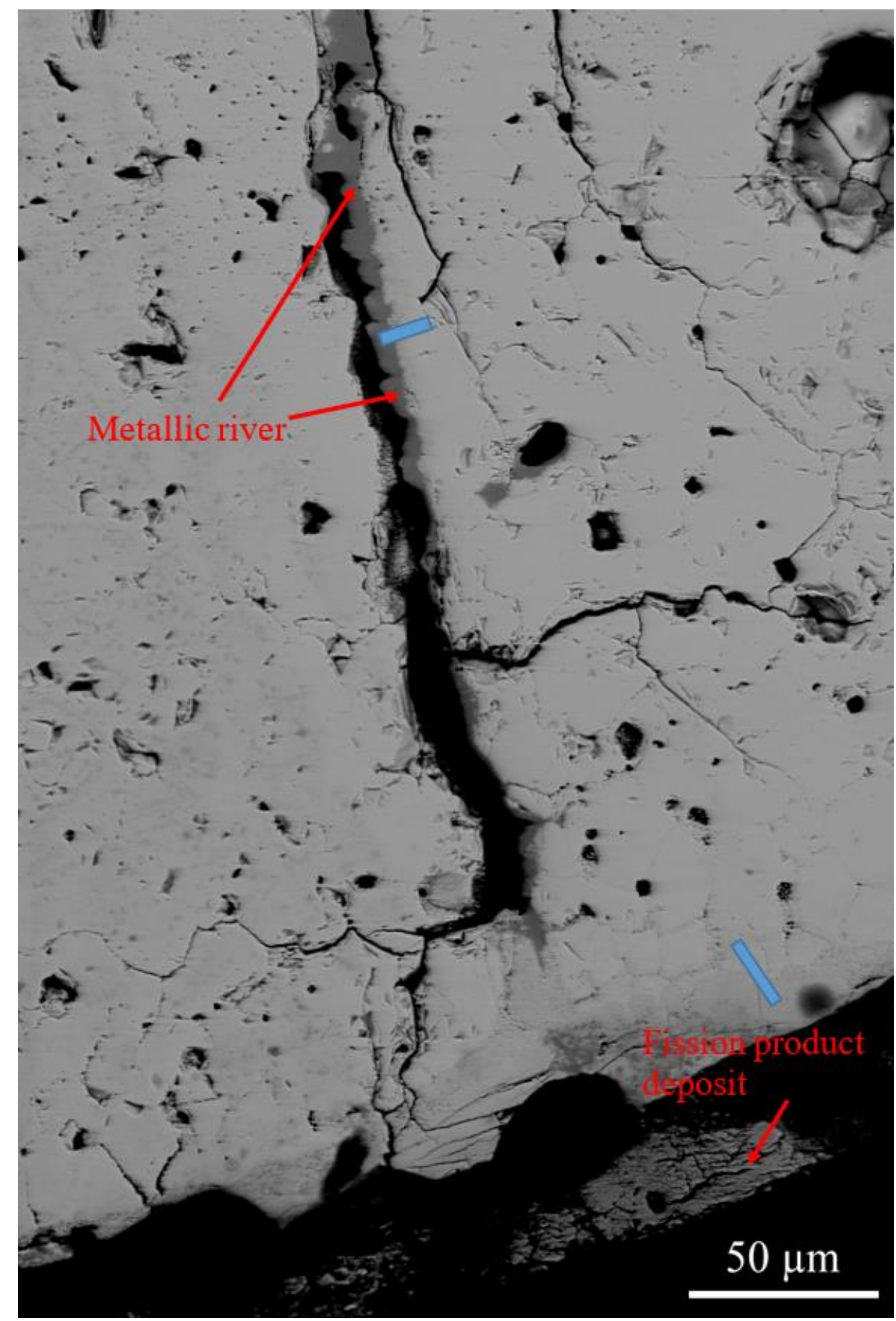

Figure 4. BSE image of the metallic river. The rectangles indicate the location of the TEM lamellae described in the next sections. 


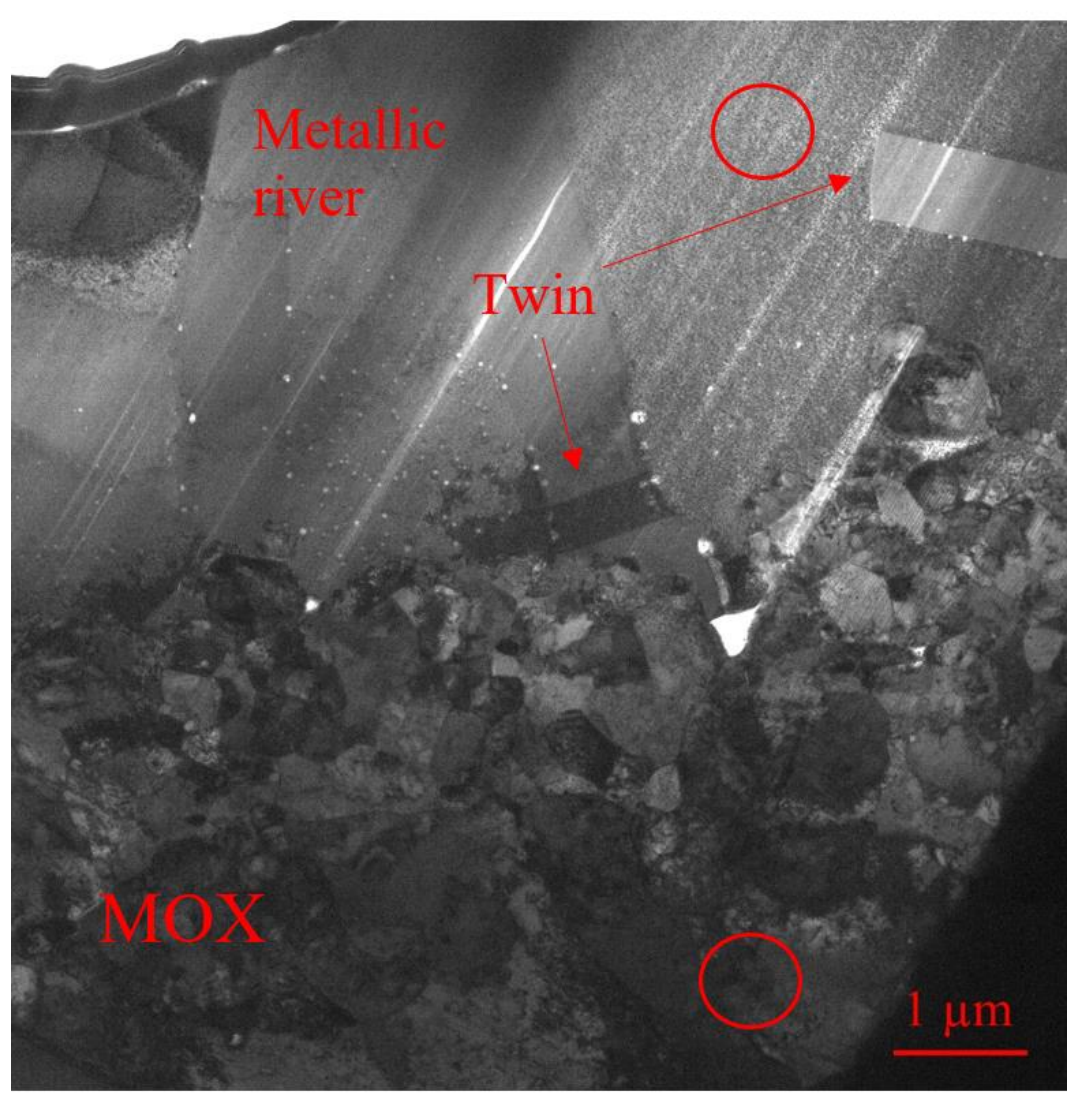

(a)

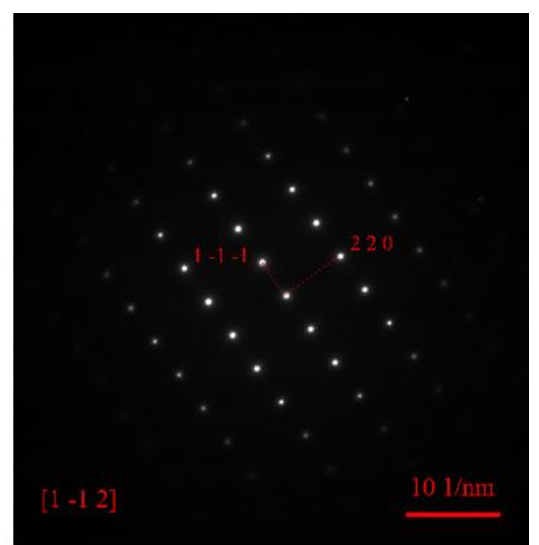

(b)

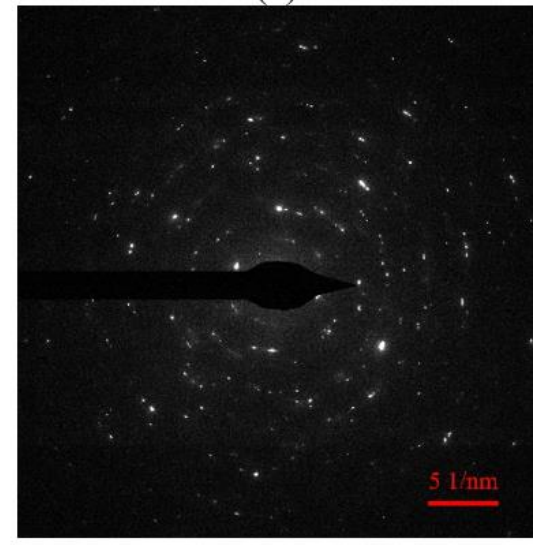

(c)

Figure 5. Bright-field TEM image of the structure at the interface between the metallic river and the MOX fuel. The red circles represent the location of the diffraction patterns shown in the insets on the right. 


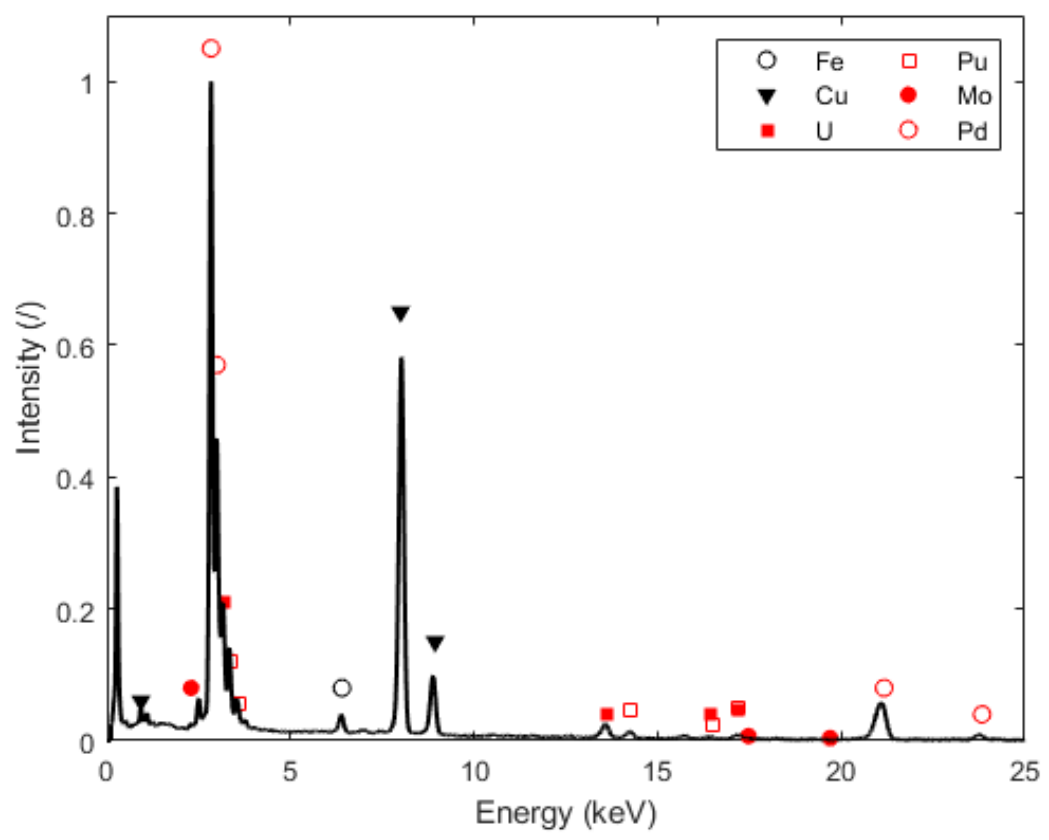

Figure 6. EDS spectrum of the metallic river shown in Figure 4. 
(a)
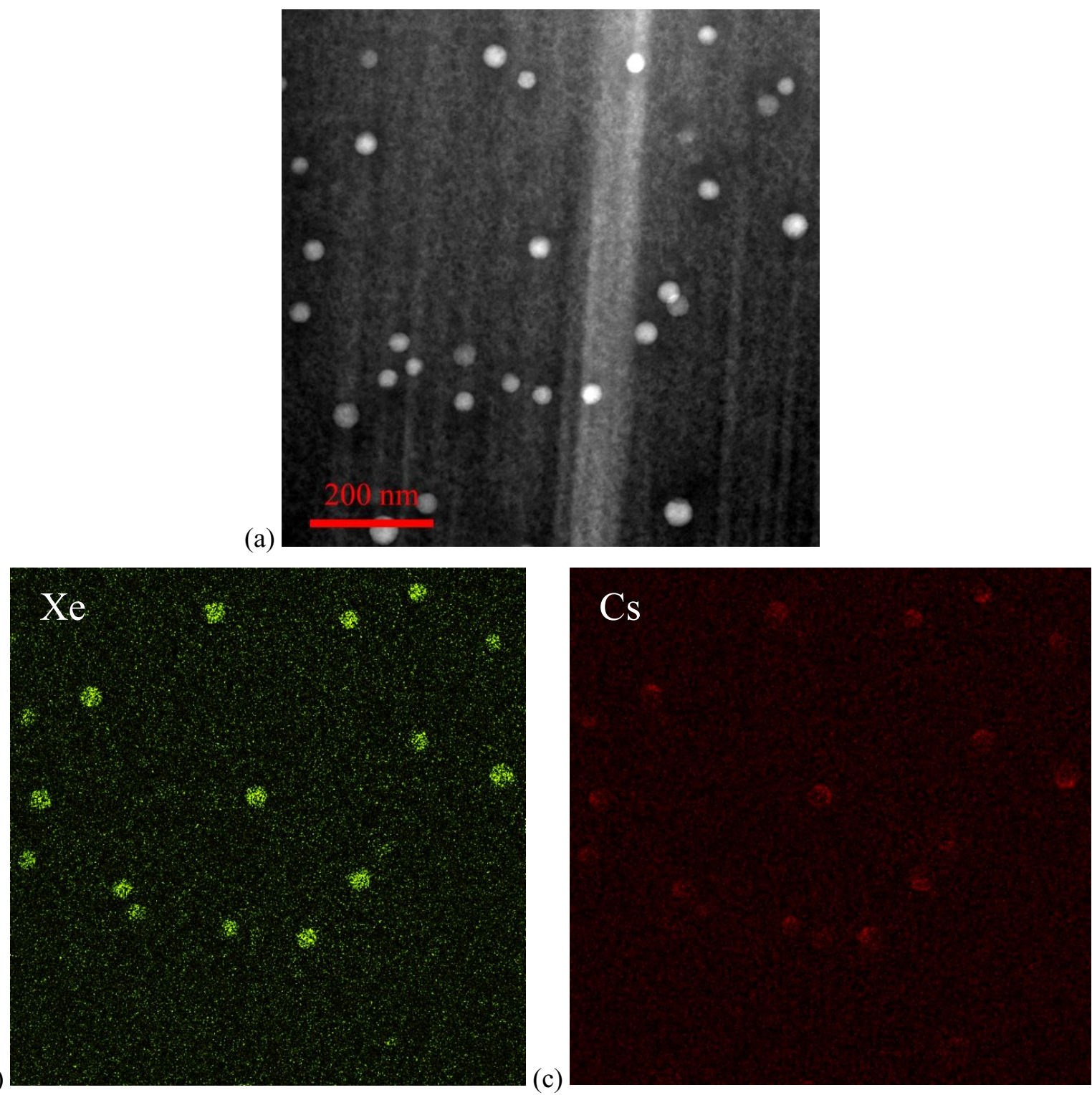

Figure 7. EDS maps (in at \%) showing the presence of fission gas bubbles in the Pd matrix. Cs accumulates on the surface of the bubbles.

At the pellet periphery initial stages of fission product precipitation in the gap had occurred. The corresponding EDS spectrum is shown in Figure 8. Qualitatively, the deposit is composed mainly by fuel $(\mathrm{U}, \mathrm{Pu}) \mathrm{O}_{2}$, with presence of some Pd, whose X-ray L edges partially overlap the U, Pu M edges. In the energy range $3.78 \mathrm{keV}$ to $4.9 \mathrm{keV}$, the L edges of $\mathrm{Cs}$, Ba and Te could be confirmed, but low energy EDS cannot clearly distinguish each single contribution. The peaks at 2.14 and $9.7 \mathrm{keV}$ are a result of the gold coating, while the minor Fe peak observed is probably from the SEM chamber, as Fe peaks intensity was the same in all the spectra. Concentration of alkali metals occurred at the outermost periphery of the fuel, as highlighted both in Figure $9 \mathrm{~b}$ and $9 \mathrm{c}$. Similar results were obtained in another peripheral location next to the river, shown in Figure 10a and 10b. In addition to the Cs-enrichment, large metallic precipitates were seen, mainly composed of Pd and Ag. WDS was used to detect $\mathrm{Pd}$ and distinguish it from $\mathrm{Ag}$ and it is shown in Figure 10c. Ag accumulated only at the outermost part of the pellet, consistent with its higher volatility compared to $\mathrm{Pd}$. 
In both Figure 9, Figure 10a and Figure 10b, a different morphology of the grain structure can be qualitatively determined from the SEM images. The grain subdivision occurring where the fission products have accumulated becomes obvious from the EBSD map shown in Figure 11. The structure is slightly different from the typical High Burnup Structure (HBS), as the micrometric pores are not present, rather, the layer is quite compact. The region has been further analyzed by TEM, and the results are shown in the next section focused on the fuel periphery and cladding region.

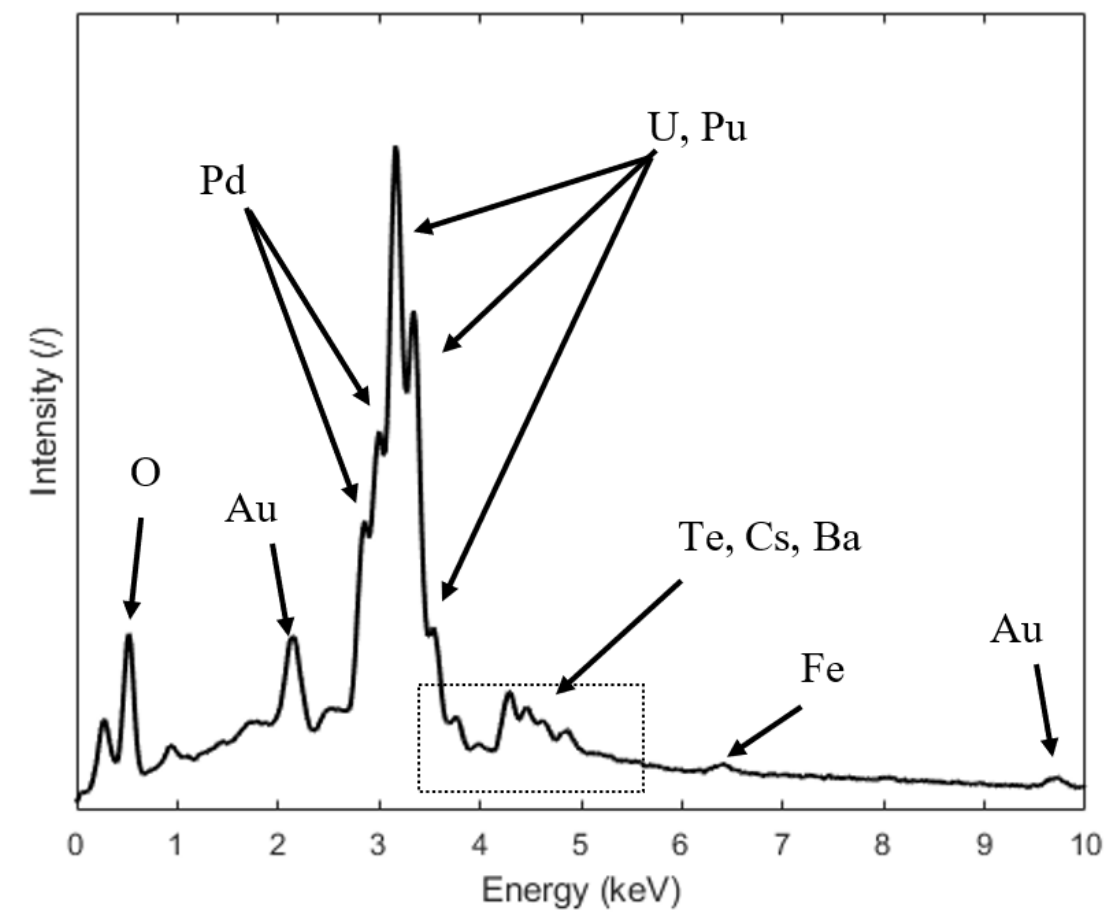

Figure 8. EDS spectrum of the fission product deposit shown in Figure 4. 


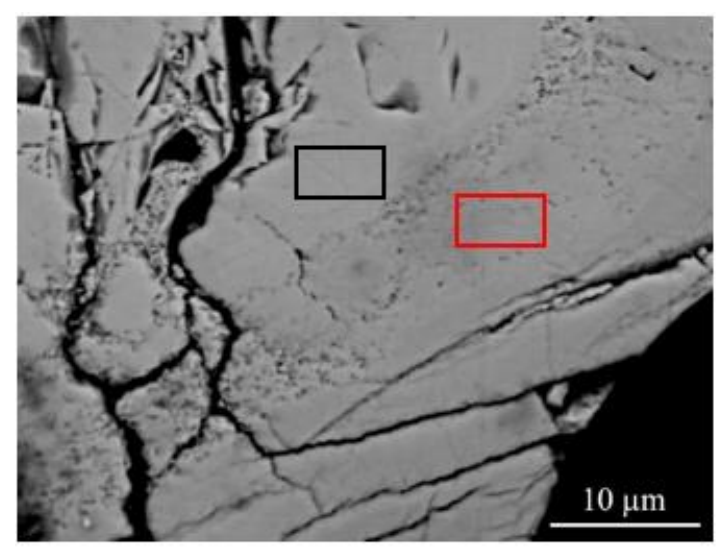

(a)

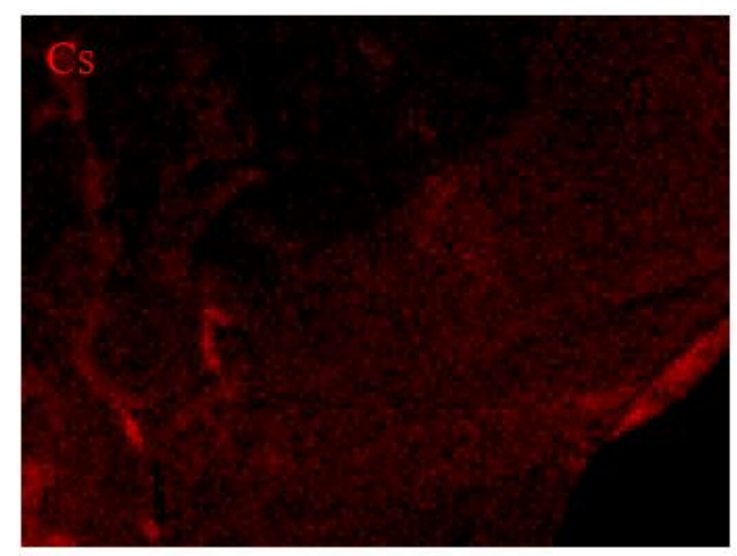

(b)

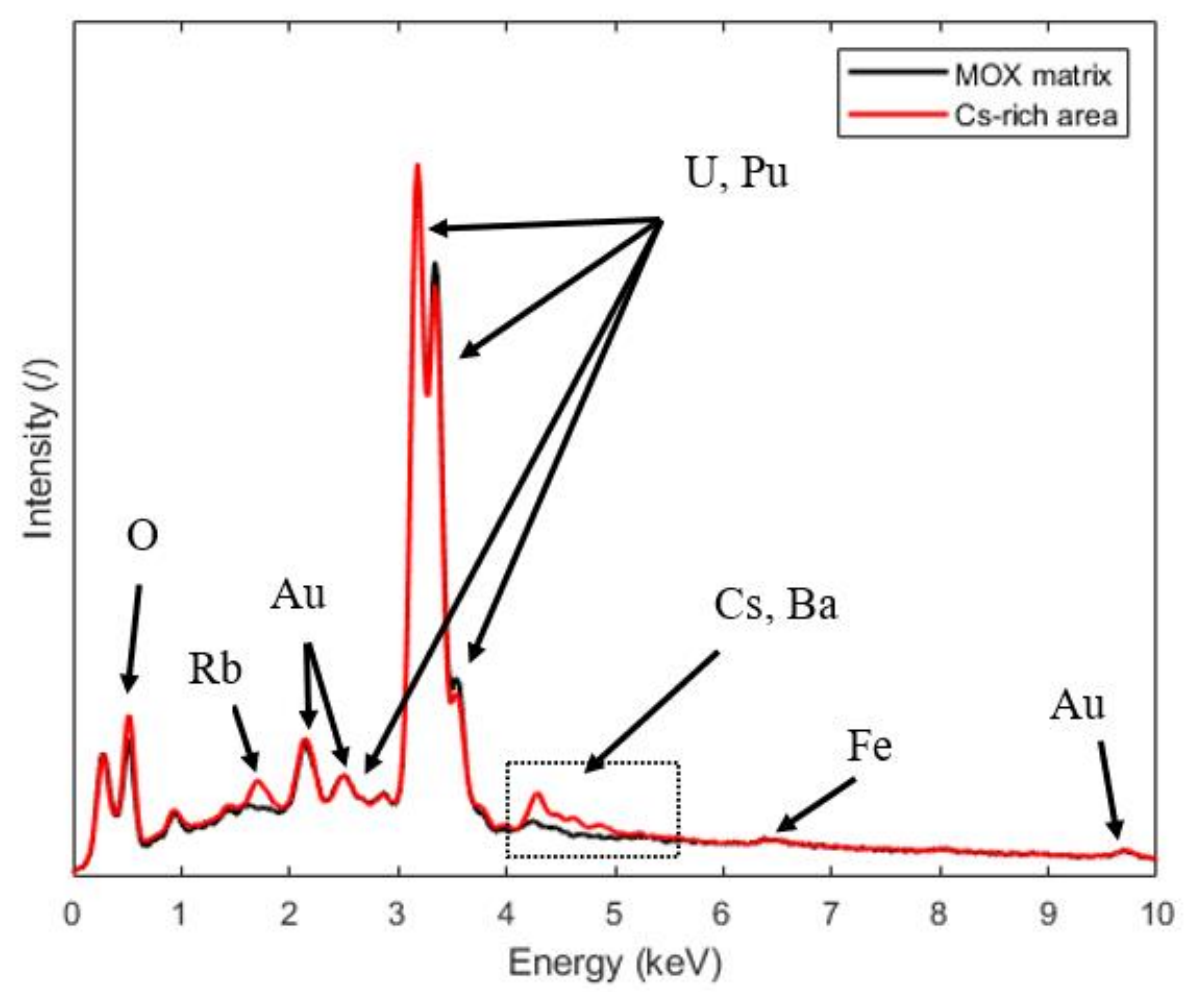

(c)

Figure 9. (a) High magnification (2500x) iimage of the outermost part of the fuel pellet at the end of the metallic river. (b) Qualitative map of Cs signal. (c) Comparison of the spectra from the boxes in (a) showing the enrichment of the alkali metals with respect to the matrix. 


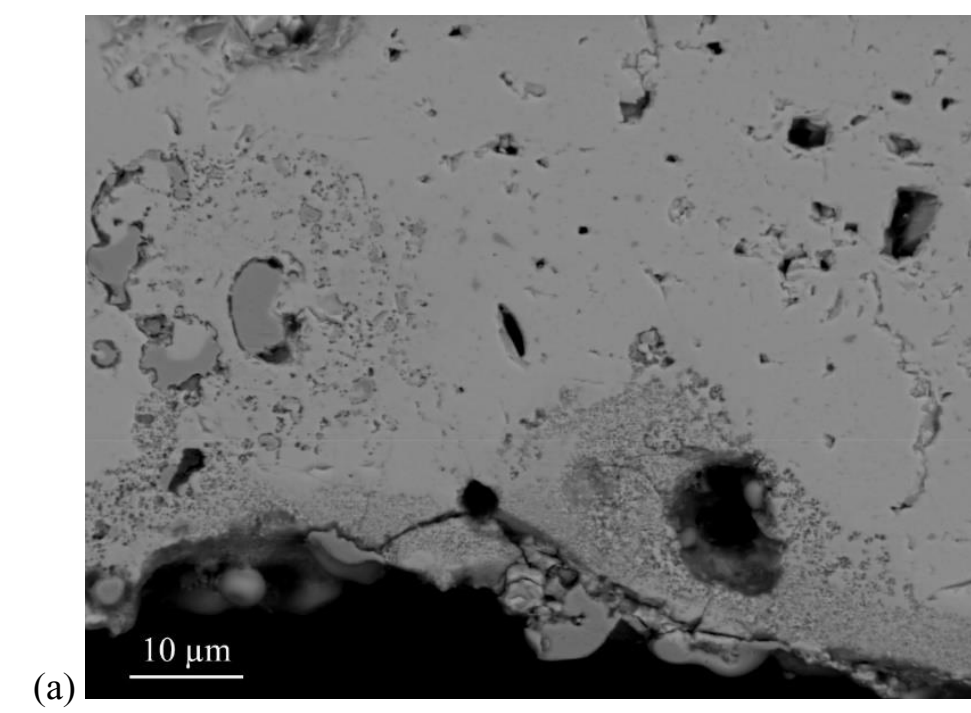

(b)
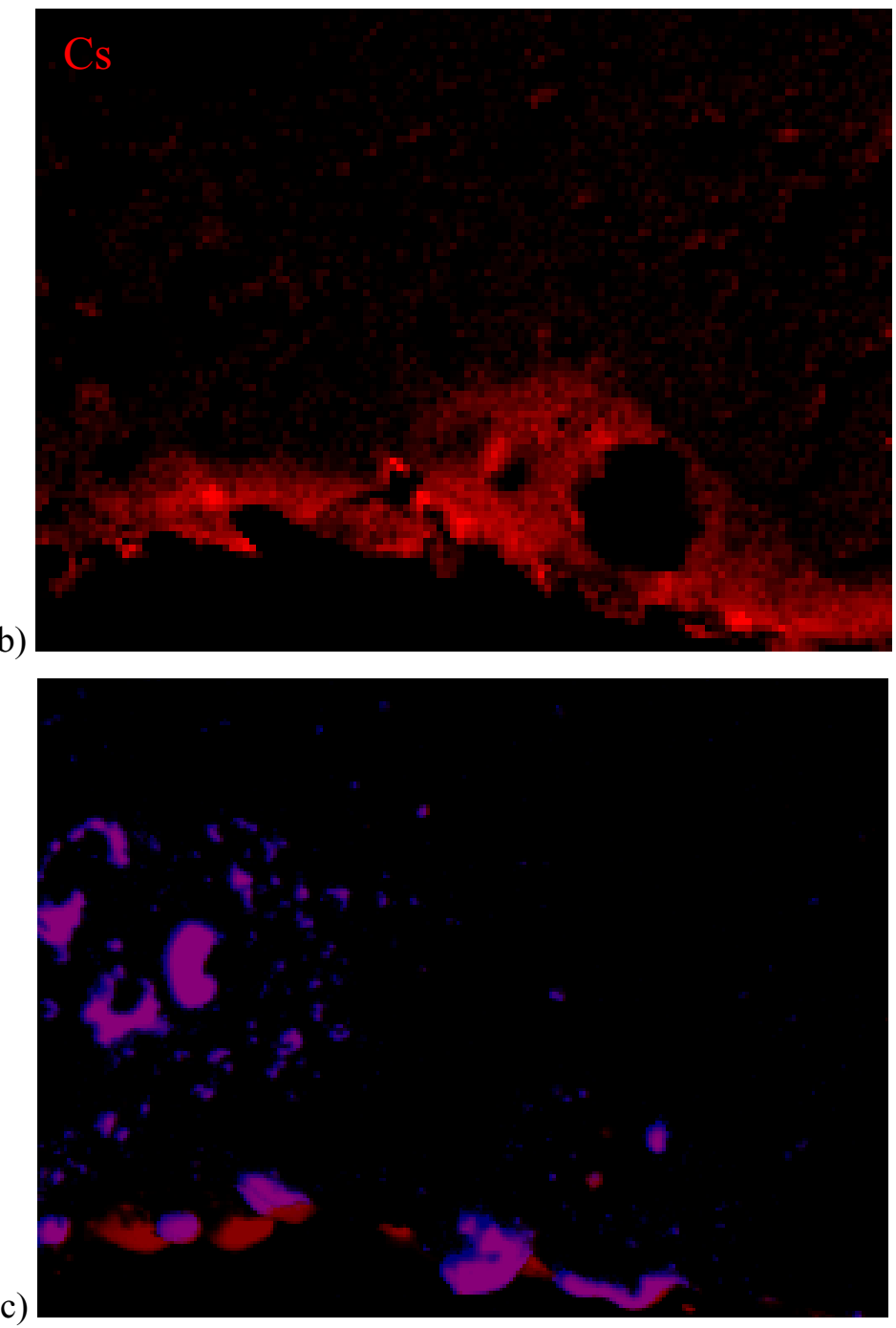

Figure 10. (a) BSE of another peripheral location adjacent to the metallic river. (b) Cs elemental qualitative map. (c) Overlay of the EDS signal from Ag (red) and the WDS signal of Pd (blue). The purple color is the result of the overlap. 

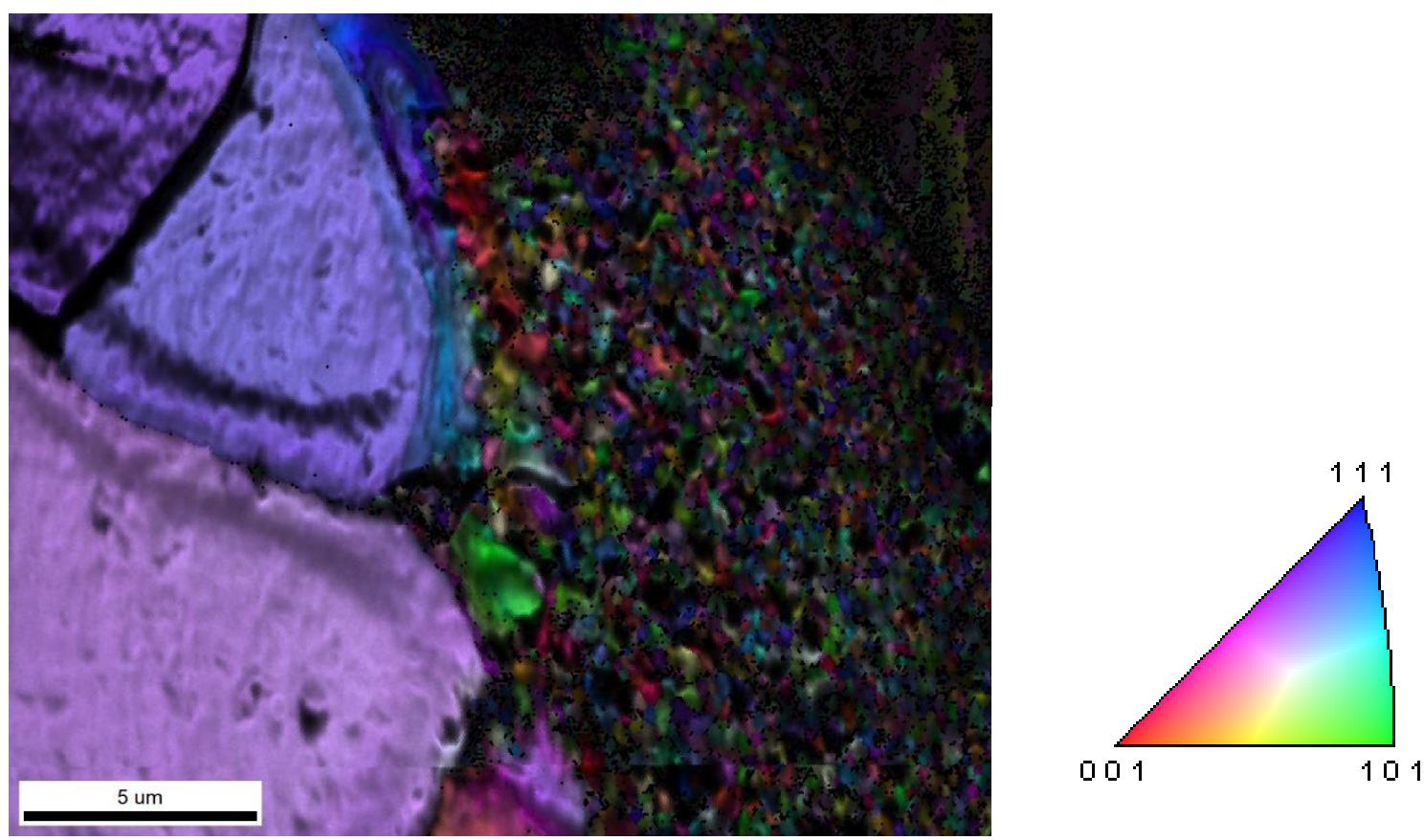

Figure 11. Inverse Pole Figure (IPF) overlaid to the image quality (IQ) map of the area at the interface between the fission product rich zone and the fuel matrix.

An additional location at the fuel periphery showed exposed grain surfaces exhibiting grooves that resemble the grain subdivision, similar to the subdivided grains of the HBS (Figure 12). EBSD mapping has been taken just outside this area (Figure 13), but no grain subdivision could be observed past the exposed fresh fracture in Figure 12. This proves that the phenomenon is restricted to that area. Some Cs enrichment was measured in the recessed zone, which suggests that a phenomenon similar to what is shown in Figure 11 has taken place. 


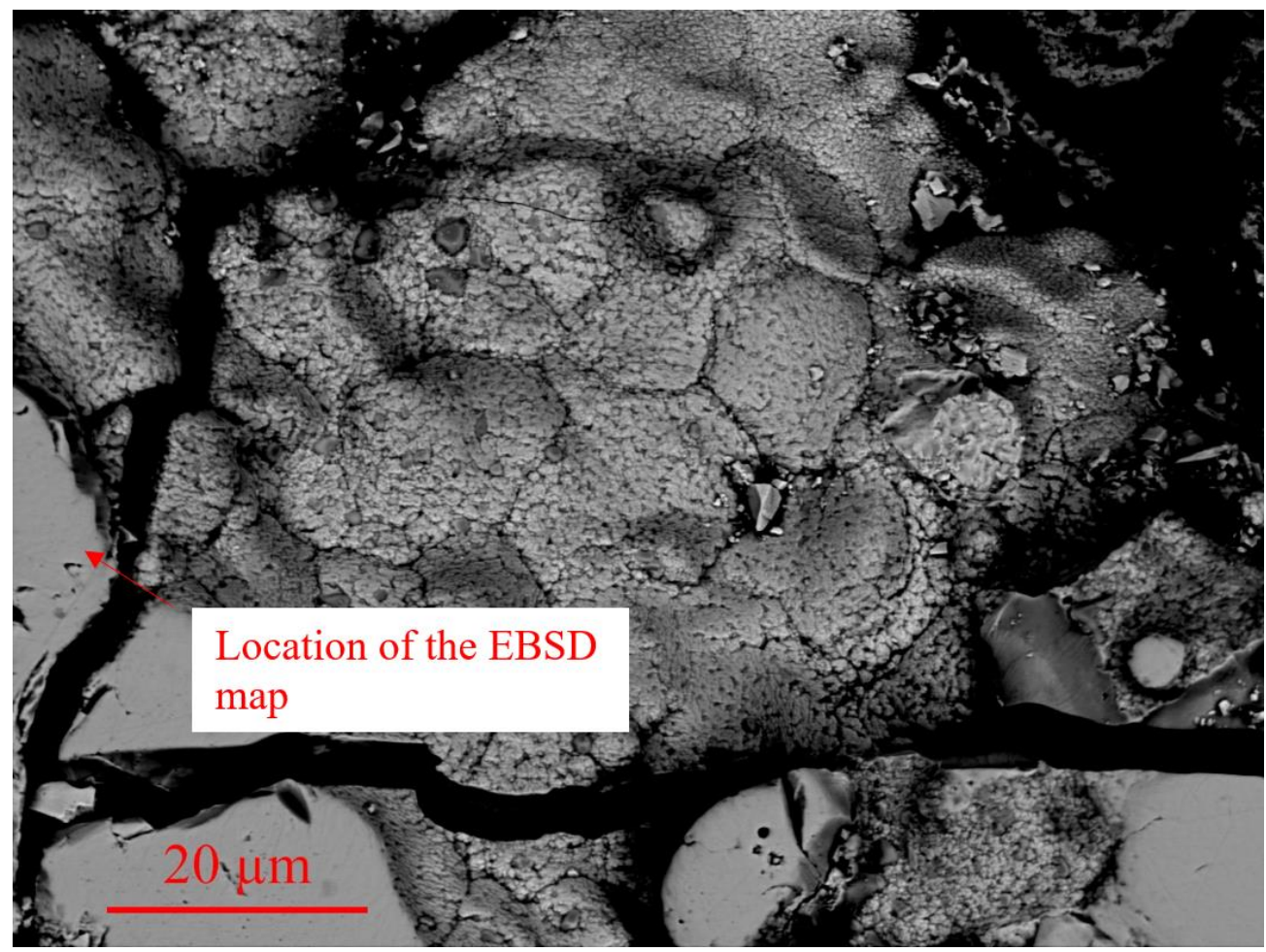

Figure 12. SEM image showing a "cauliflower-type" structure at the outermost fuel periphery.
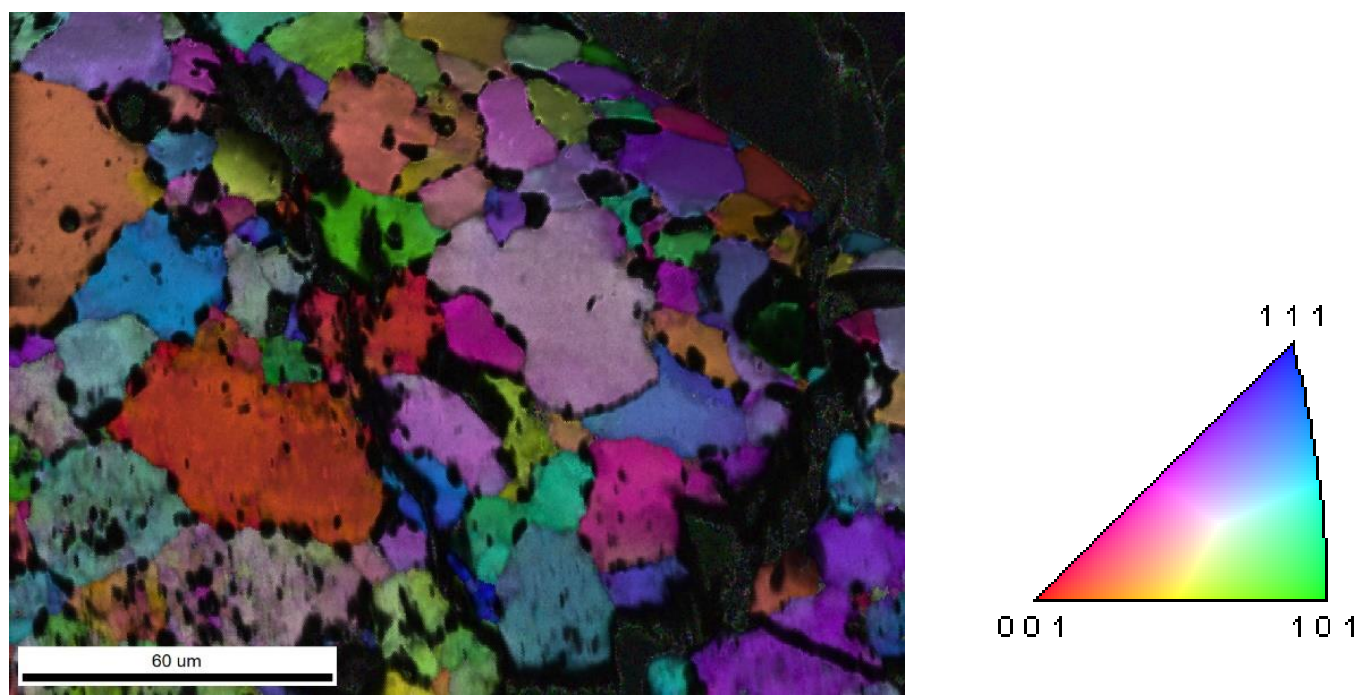

Figure 13. Inverse Pole Figure (IPF) overlaid to the image quality (IQ) map of the area outside the "cauliflower" shown in the previous figure.

\subsubsection{Fuel-cladding chemical interaction}

The entire periphery of the annular fuel was examined. The common observation of the various inspections was the presence of fractured fuel pieces partially attached to the cladding, containing different amounts of fission products. Examples are reported in Figure 14, Figure 15, and Figure 16a from boxes 2, 3 and 4 in Figure 1a, respectively. The BSE image in Figure 14 shows a slightly darker fuel color along the grain boundaries and on the outermost part of the pellet. As the BSE contrast is sensitive to the atomic number of the material, it means that lighter phases are present. Figure 15 shows a fracture 
surface. The irregularity of the surface and its depth did not allow the gathering of EDS data, butit is apparent, from the surface morphology, that grain subdivision has occurred. The most interesting deposit was formed in area 4, showing a considerable amount of Cs (Figure 16c) and spots rich in Te close to the cladding (Figure 16d). Pd appears mostly deposited on the internal crack surface, and only spots in the interaction layer show some Pd enrichment, visible as dots in Figure 16e. In two locations, intergranular corrosion of the HT-9 has initiated (Figure 17 and Figure 18a). Cs and Te are associated with the corroded area, confirming the historical results regarding the influence of Te and Cs on the cladding corrosion process [12-15].

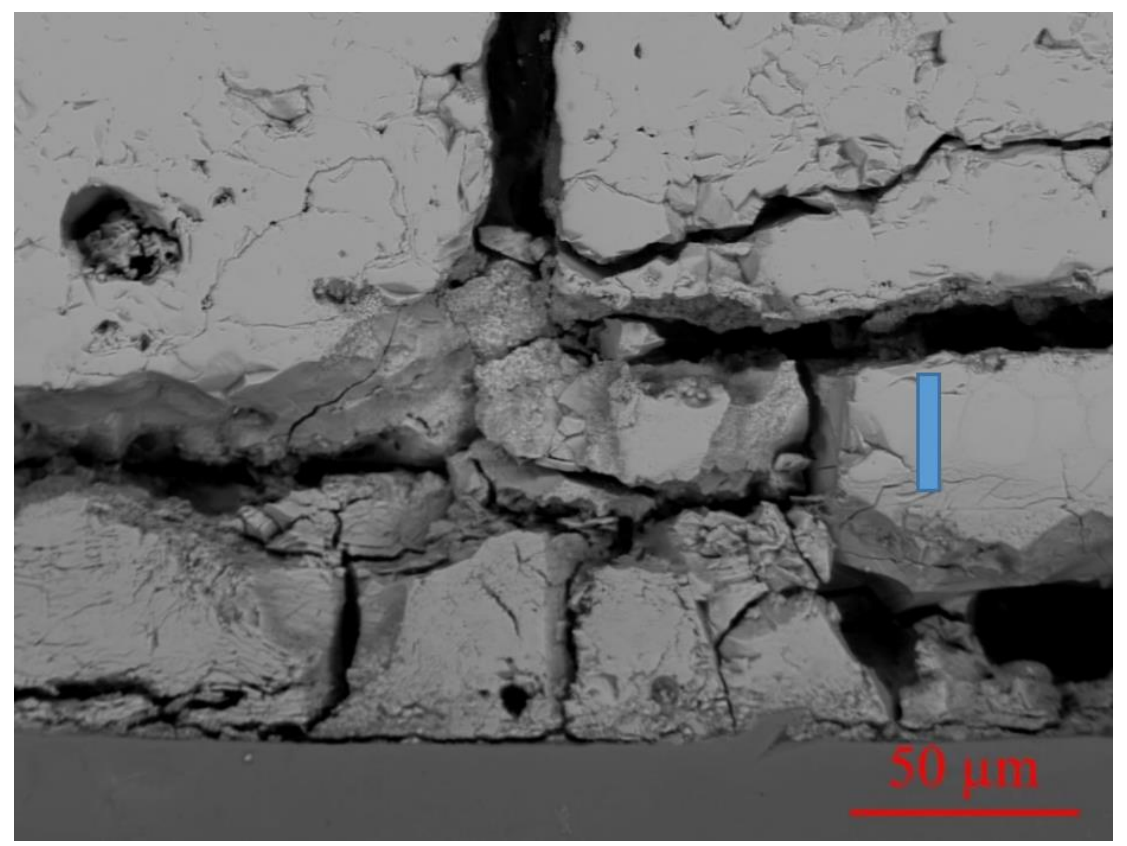

Figure 14. BSE image of the fuel to cladding region from area 2 (from Figure 1a). The rectangle indicates where the TEM lamella was extracted.

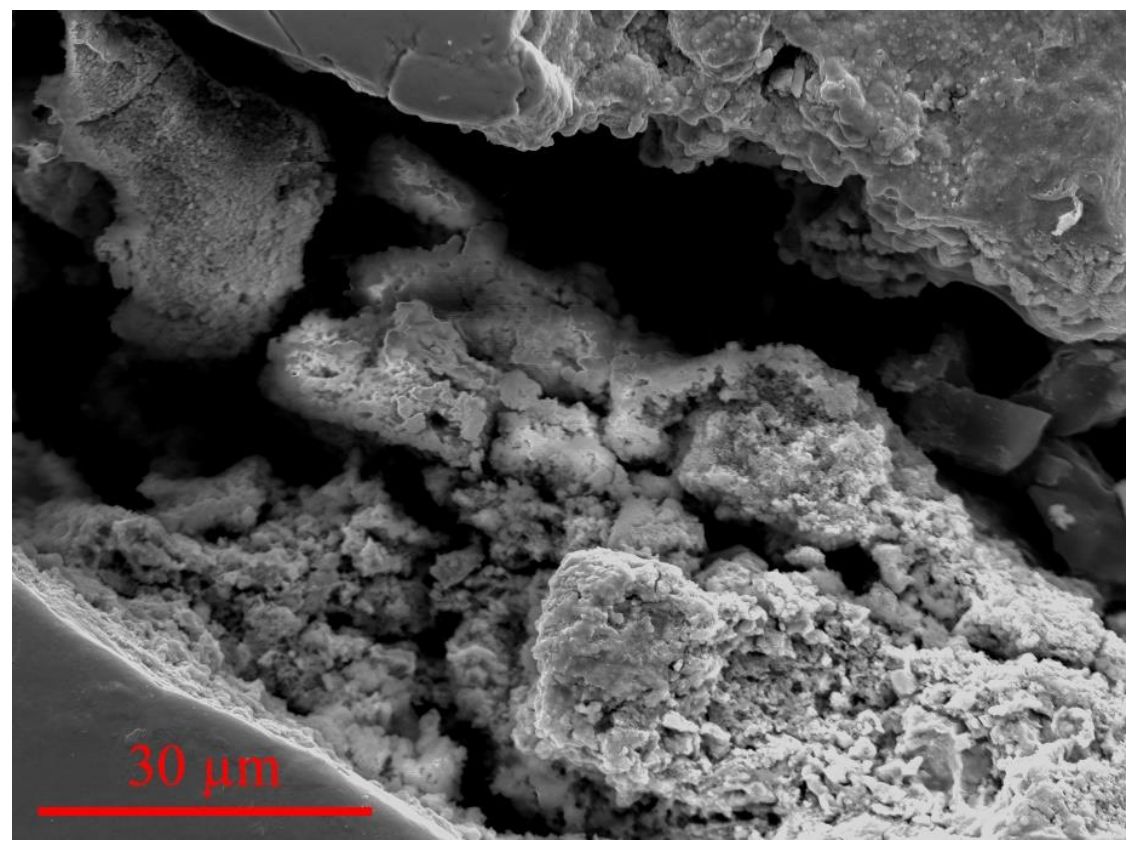

Figure 15. SE image of the fuel to cladding region from area 3 (from Figure 1a). 


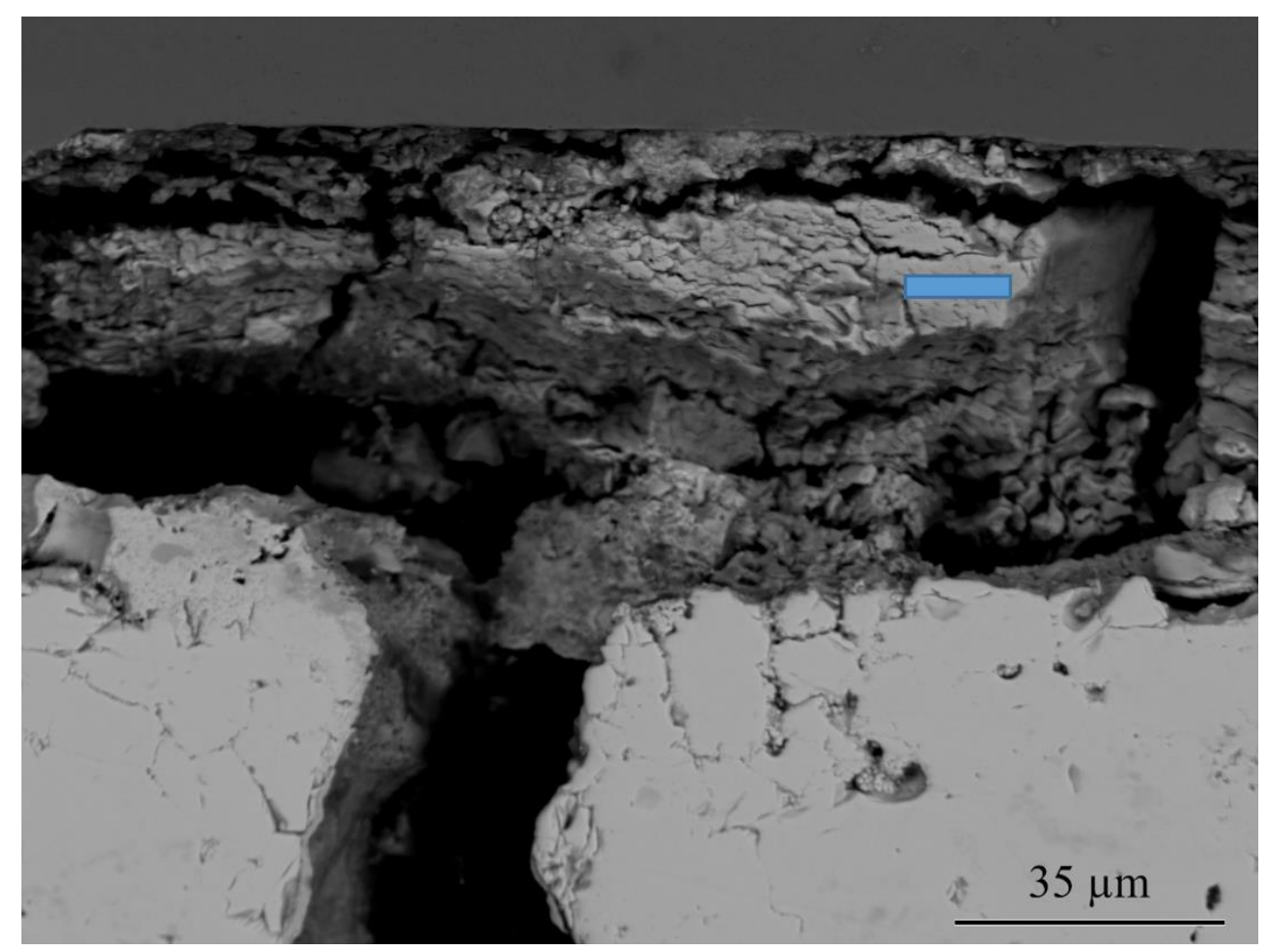

(a)
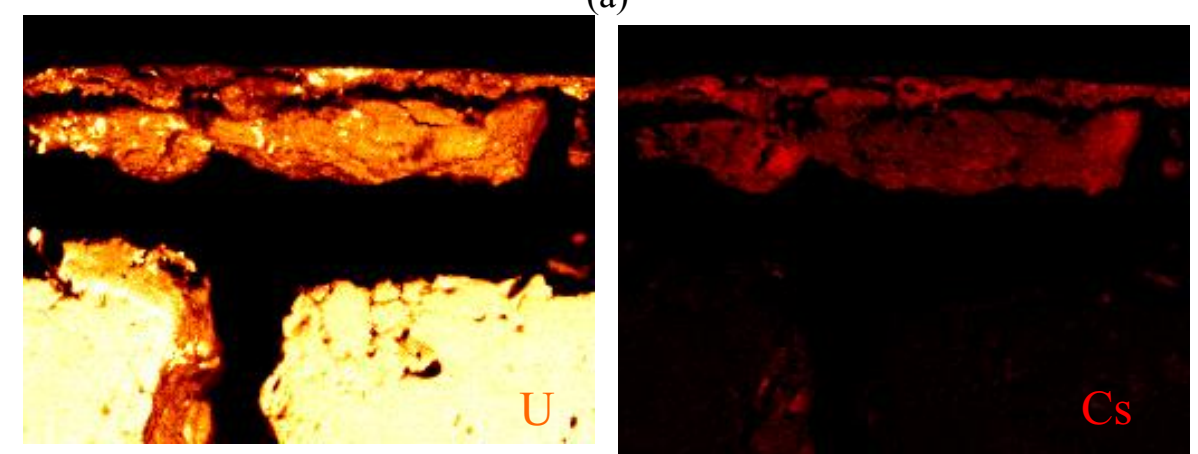

(b)

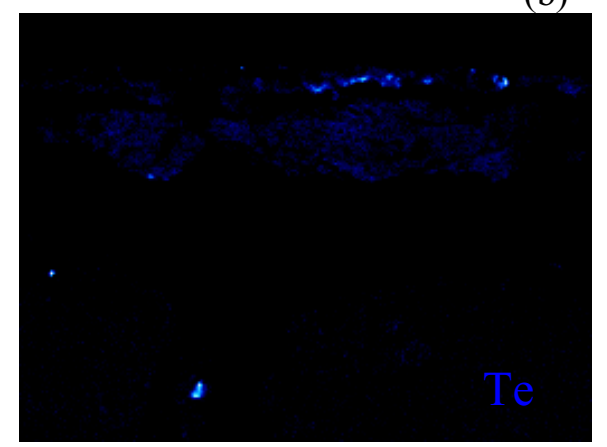

(c)

(d)

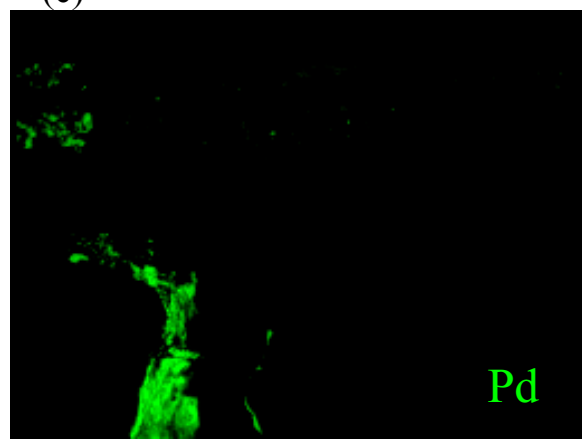

(e)

Figure 16. (a) BSE image of the fuel to cladding area from area 4 in Figure 1a. (b)-(e) EDS maps of fuel major component and fission product. The rectangle in (a) indicates where the TEM lamella was extracted. 


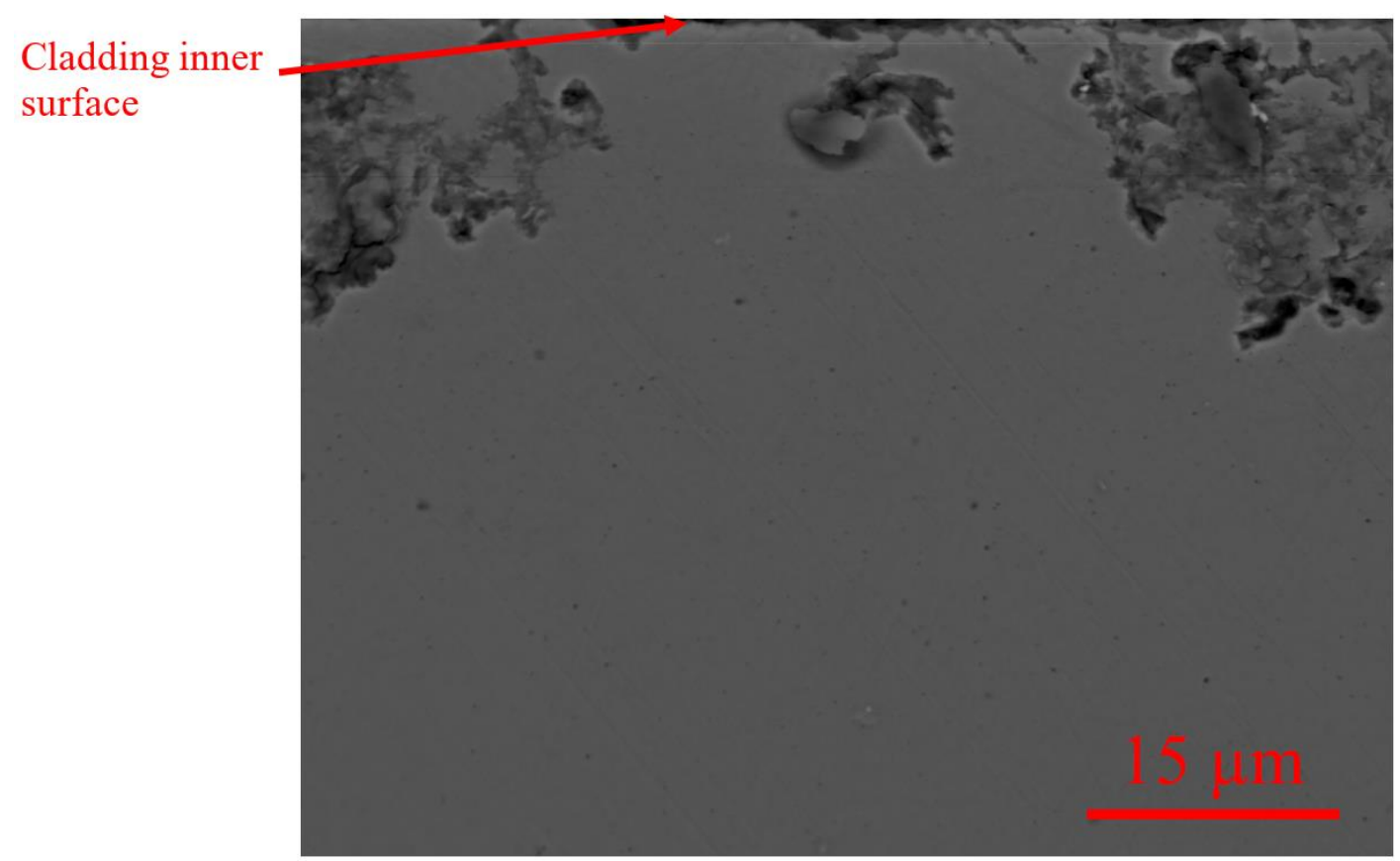

Figure 17. Overview of intergranular corrosion in the HT-9 cladding.

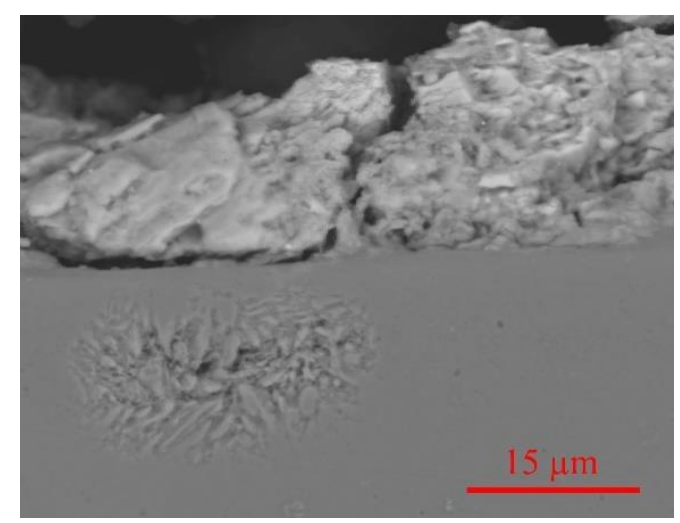

(a)

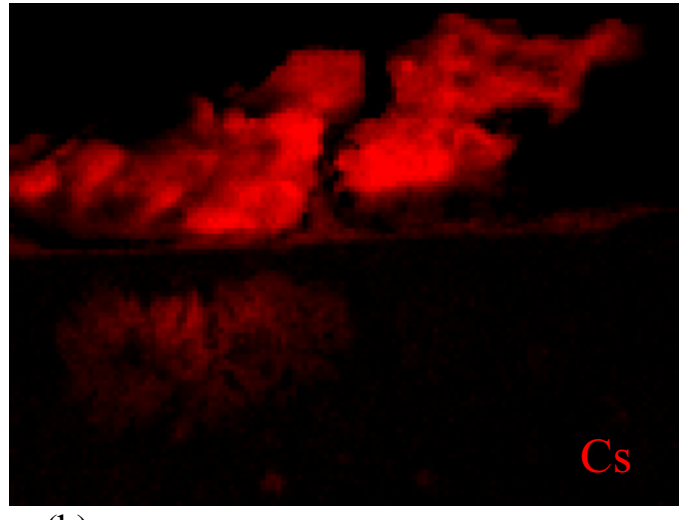

(b)

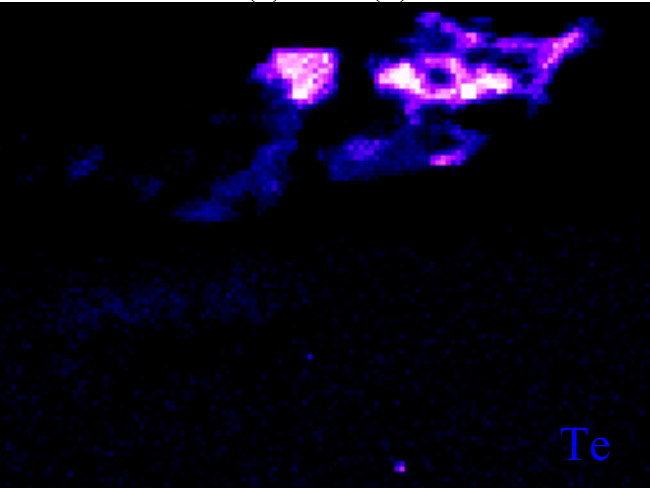

(c)

Figure 18. Other location in the HT-9 cladding with initial stages of corrosion. Cs and Te are associated with the corroded area. 
TEM analyses focused on the fuel side only. TEM lamellae were extracted at the locations of the boxes in Figure 14 and Figure 16a. The SEM/EDS maps showed previously (e.g., Figure 9b and Figure 16b-e) indicate that the majority of the corrosive fission products is still retained in the outermost portion of the fuel matrix, hence, it is necessary to provide details regarding the fuel chemical composition and structure in these regions. Low magnification overviews of the lamellae are reported in Figure 19. The three lamellae were extracted from locations with distance of $\approx 70 \mu \mathrm{m}, \approx 55 \mu \mathrm{m}$ and $\approx 18$ $\mu \mathrm{m}$ from the cladding inner surface, respectively. 


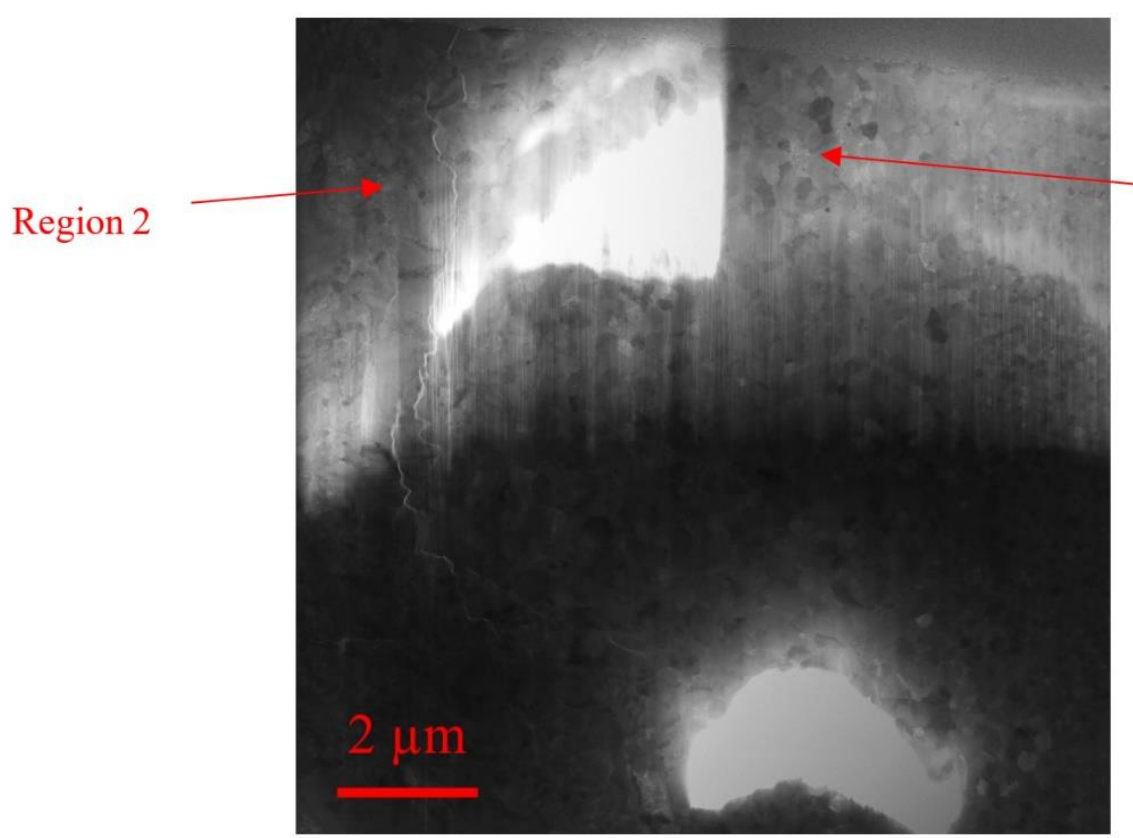

Region 1

(a)

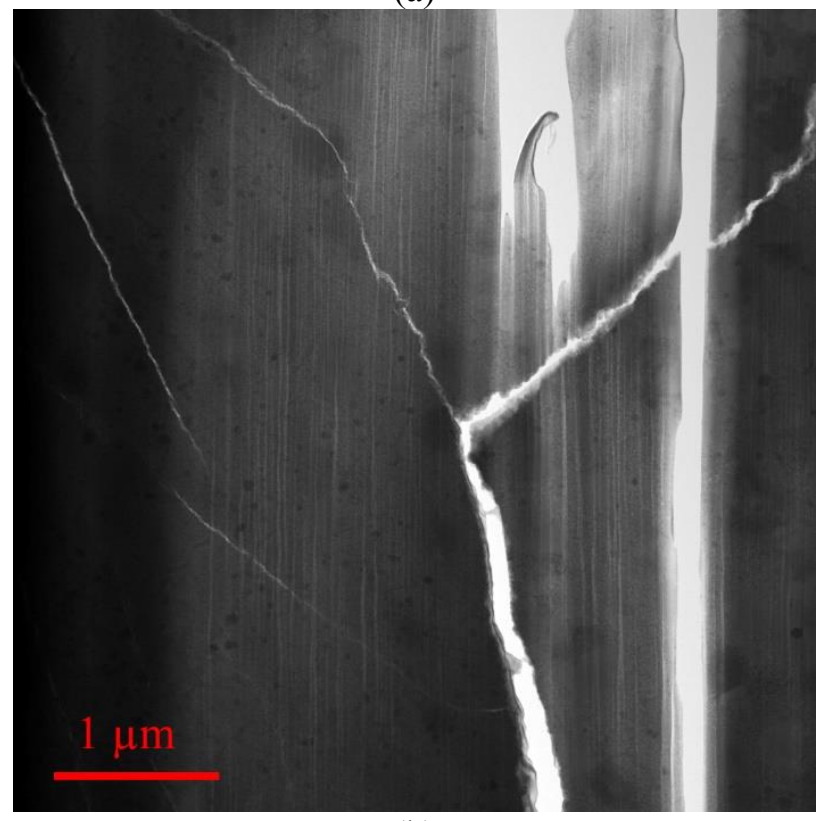

(b) 


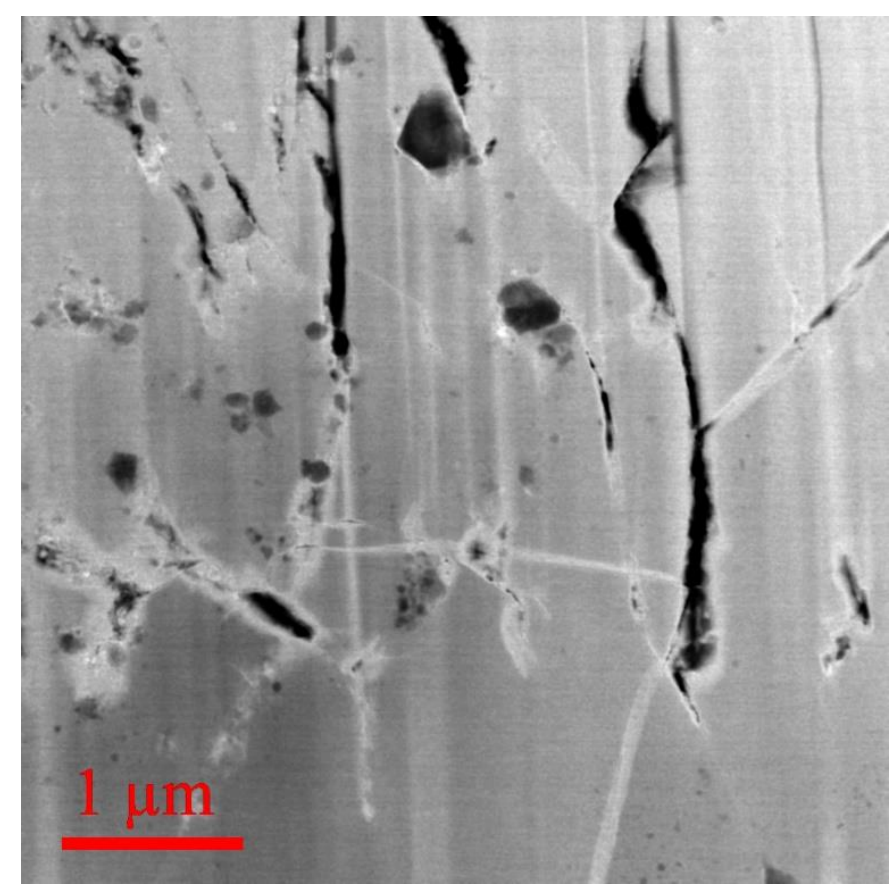

(c)

Figure 19. Low magnification bright field images of the lamellae. (a) Lamella from the fuel periphery close to the metallic river. (b) Lamella from FCCI area 2 in Figure 1a. (c) Lamella from FCCI area 4 in Figure 1a.

It is obvious from the overview in Figure 19a that the majority of fuel grains have subdivided, and they are in the sub-micrometric range, which was also shown through the EBSD map in Figure 11. High magnification images (see Figure 20) in two areas show fully recrystallized grains in the center of the lamella (region 1 in Figure 19a) and more elongated grains still in the sub-micron range on one side (region 2 in Figure 19a). Intergranular micro-cracking is visible next to region 2. Semi-quantitative EDS analyses showed that the region is mainly composed of $\mathrm{U}, \mathrm{Pu}, \mathrm{O}$, with $\mathrm{Cs}$ and the daughter $\mathrm{Ba}$ accounting for approximately $2 \%$ of the atomic composition (see spectrum in Figure 21). Metal precipitates containing $\mathrm{Pd}, \mathrm{Ru}, \mathrm{Rh}$ but no Mo or Tc were scattered throughout the matrix.

Diffraction Electron Precession (DEP) data have been collected in both regions and analyzed assuming a cubic Fm $\overline{3} \mathrm{~m}$ structure of MOX [16]. In region 2 of Figure 19a, the proximity to the TEM grid attachment with possible local bending of the lamella made the data refinement uncertain, with a very low confidence index and poor image quality. Good results were obtained for the central region 1. Processed data and grain statistics are shown in Figure 22. The majority of the grain boundaries is of high angle nature (i.e., $>15^{\circ}$ as seen from Figure $22 a$ ), suggesting a recrystallization mechanism. The average grain equivalent diameter peaks around 100 and $300 \mathrm{~nm}$ (Figure 22b). In Figure 22c the Grain Orientation Spread $^{1}$ (GOS) map reveals thatthe level of strain is overall limited within the grains, supporting the theory that the grains are recrystallized. The values are similar to the measurements of grain size performed by Ray et al. [17] in high burnup structure of $\mathrm{UO}_{2}$ fuel. The burnup accumulation at this axial location is just above $50 \mathrm{GWd} / \mathrm{tHM}$, which is the lower bound reported for the formation of High Burnup Structure (HBS) in Light Water Reactor (LWR) fuels [18]. Hence, it can be concluded that the outermost periphery of the pellet has experienced a transformation similar to the HBS grain formation. However, one major difference occurred. As previously mentioned, no large micrometric pores, typical of the HBS

\footnotetext{
${ }^{1}$ For each grain in the mapped area, the GOS measured the degree of orientation change between every pixel in the grain and the grain's average orientation.
} 
[19-21], have formed. The SFR indeed have a much larger fission gas release compared to LWR fuels occurring relatively early in life [22]; hence, it can be expected that less closed porosity could have formed, having the gas been released at the initial stage of irradiation. The question remains whether the accumulation of the fission products, particularly $\mathrm{Cs}$, has caused the recrystallization process. HBS formation has already been observed on the outermost part of the SFR pellets [23]. SFR fuel does not show the typical increase in local fission density on the periphery originating the HBS formation; instead, the recrysatllization is confined to this area by the irradiation temperatures [22]. By observing how the structure transitions abruptly from the original micrometric grains to the new structure and how well this correlates to the presence of Cs, it can be concluded that, although Cs might not be the cause of the restructuring, the presence of Cs favors the structure modifications. Cesium uranate compounds are known for viscoplastic behavior at reactor temperature [24], which could have aided the microstructure reorganization. 


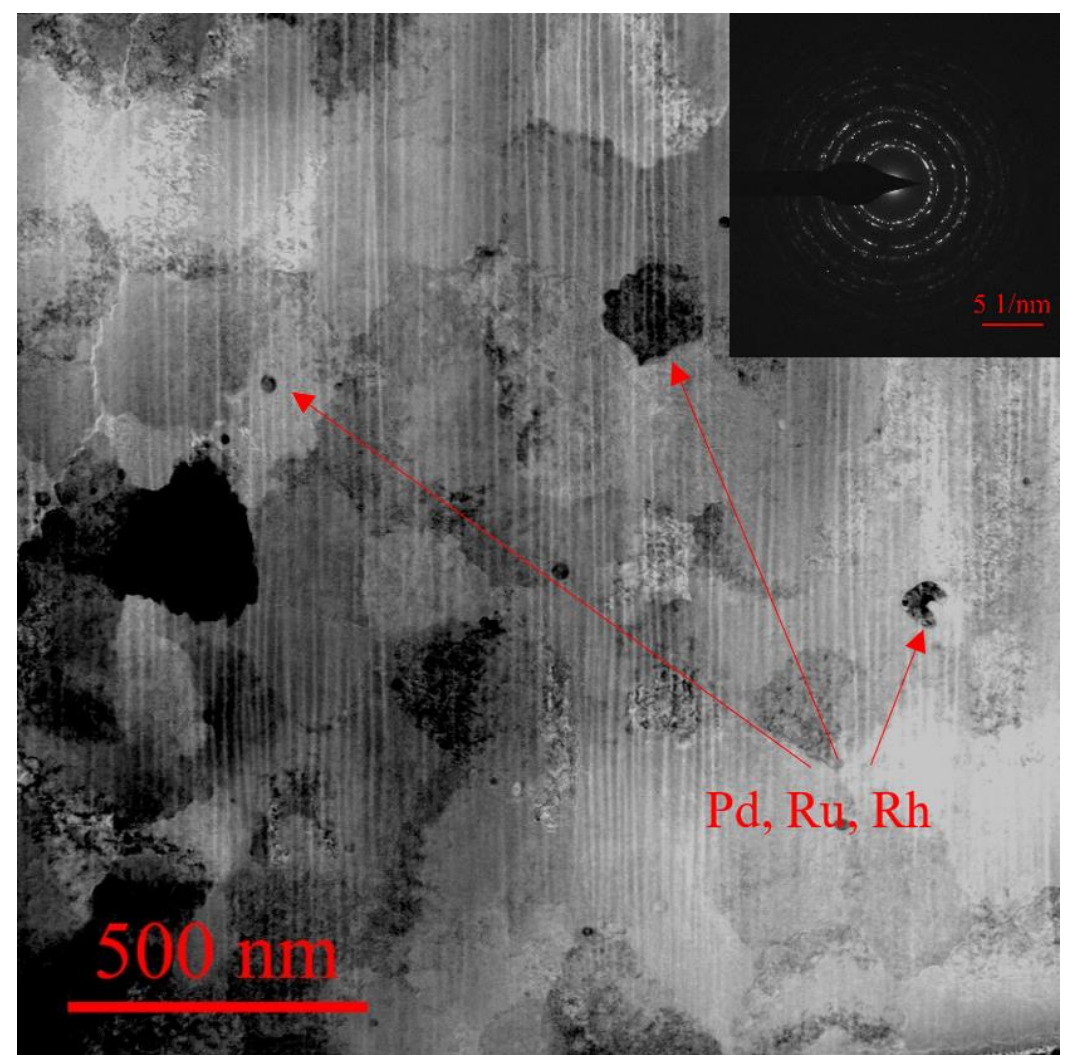

(a)

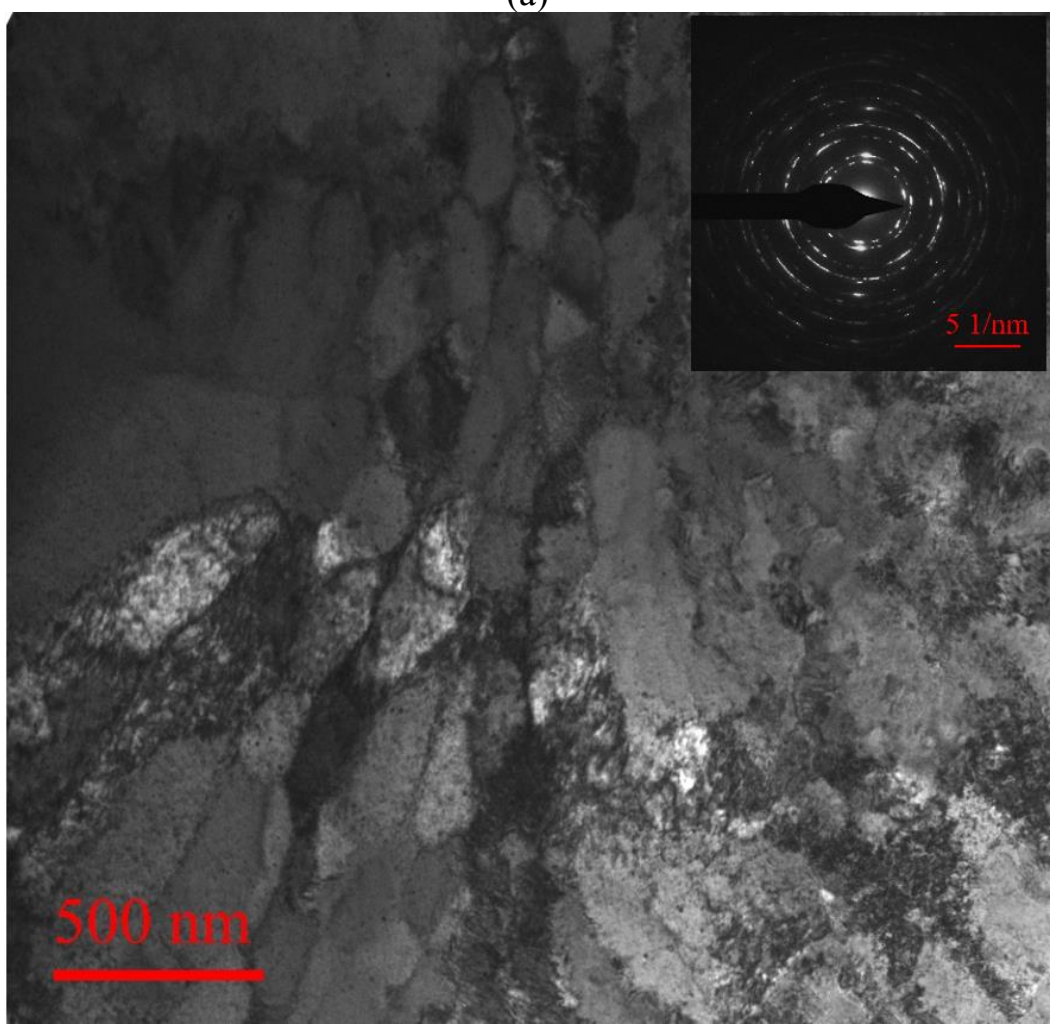

(b)

Figure 20. High magnification images corresponding to (a) region 1 and (b) region 2. 


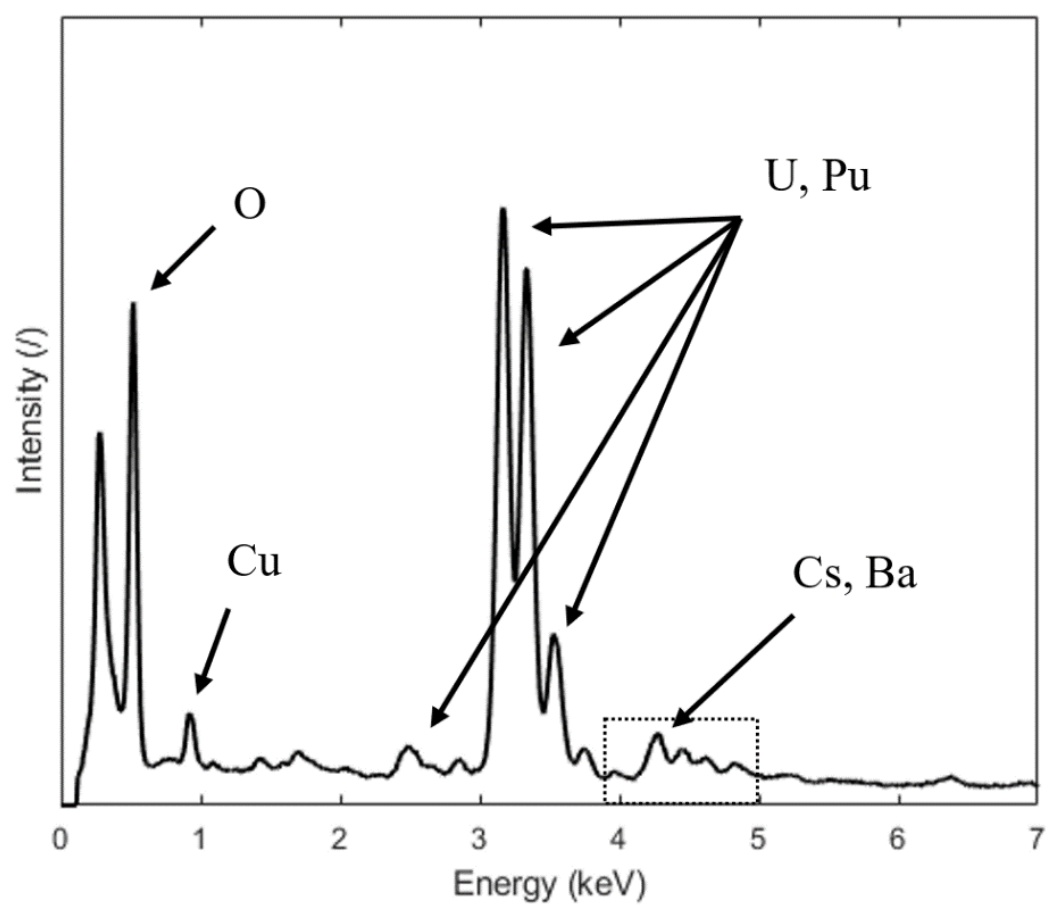

Figure 21. EDS spectrum showing the main components of the matrix. 


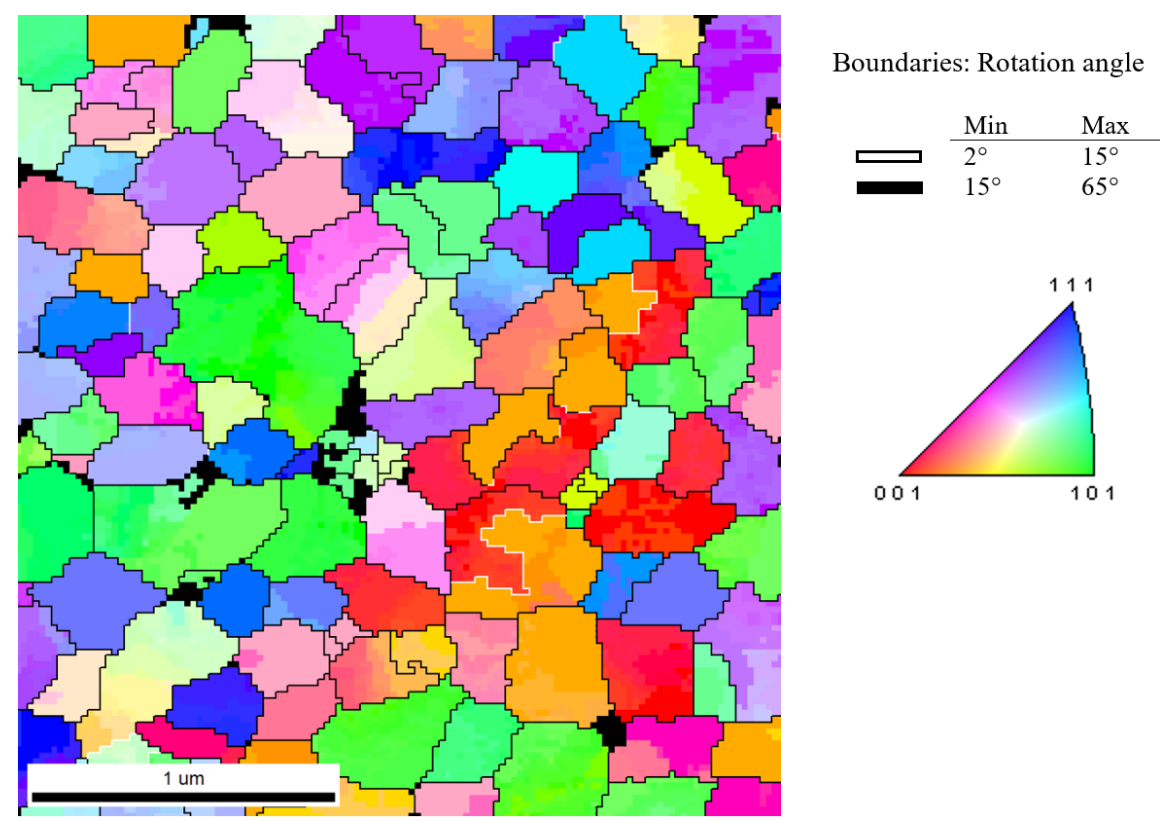

(a)

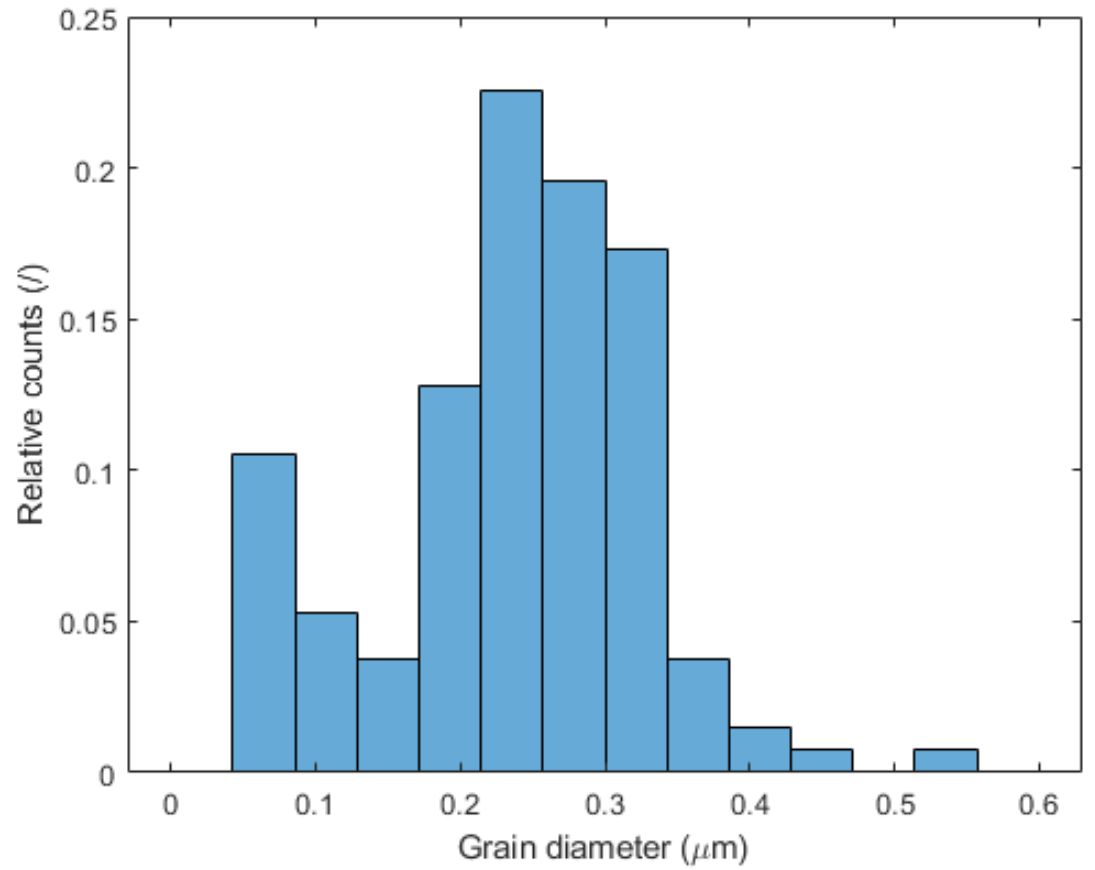

(b) 

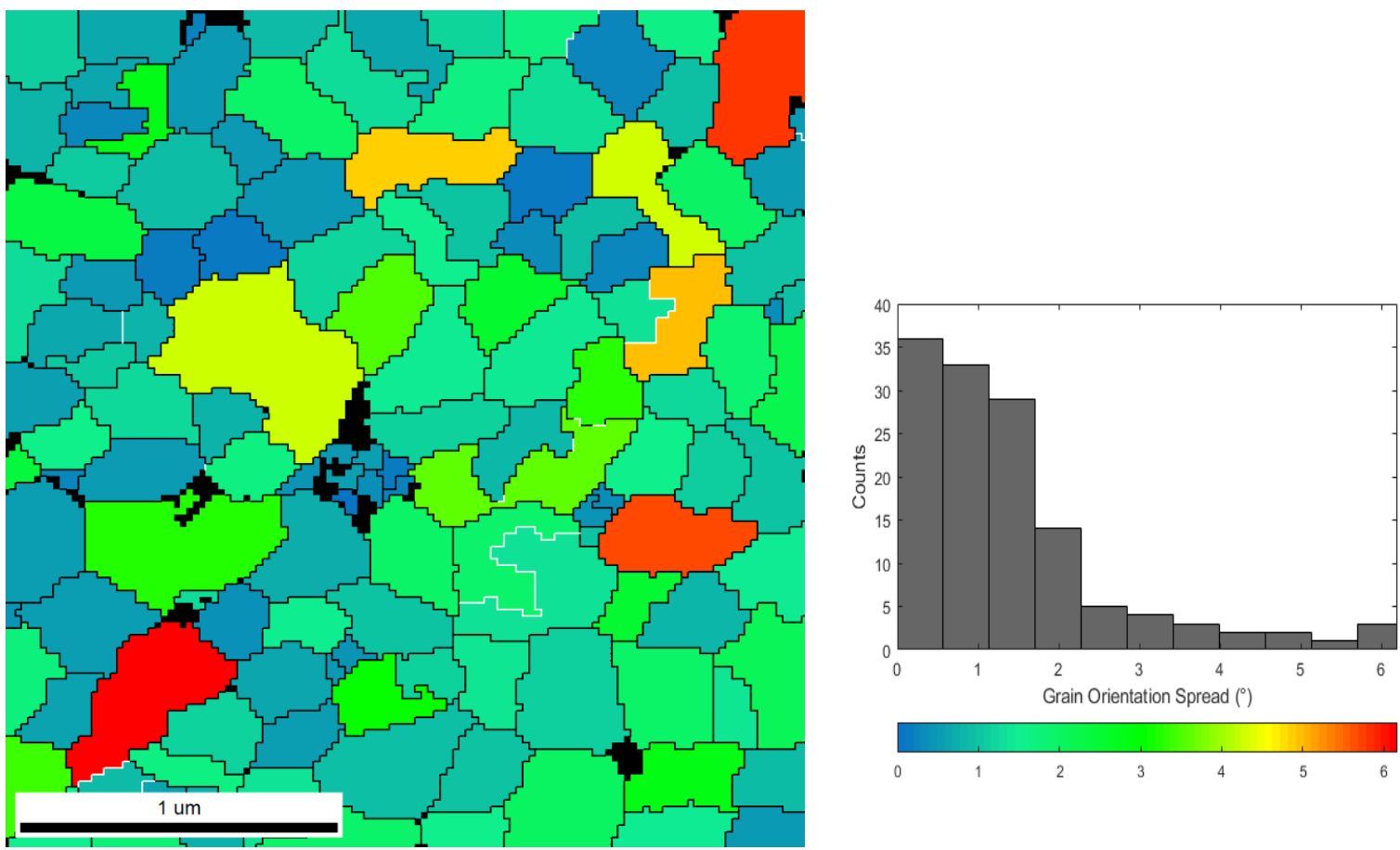

(c)

Figure 22. DEP data from region 1. (a) Inverse Pole Figure (IPF) and grain boundaries misorientation. (b) Grain diameter distribution. (c) Grain Orientation Spread (GOS) map and distribution.

Moving towards the cladding, the analysis of the second lamella in Figure 19b gave similar results to the previous lamella. The matrix is largely nanocrystalline, but isolated grains could also be mapped, whose diffraction pattern agrees with a cubic structure (Figure 23). However, some patterns could not be fitted with the MOX fluorite structure. The amount of fission products increases, in particular some grains are more rich in Cs, as can be seen from Figure $24 \mathrm{~b}$. Figure 25 shows Te deposition preferentially along the cracks. No cladding components were found associated with Te or other fission products at this distance.

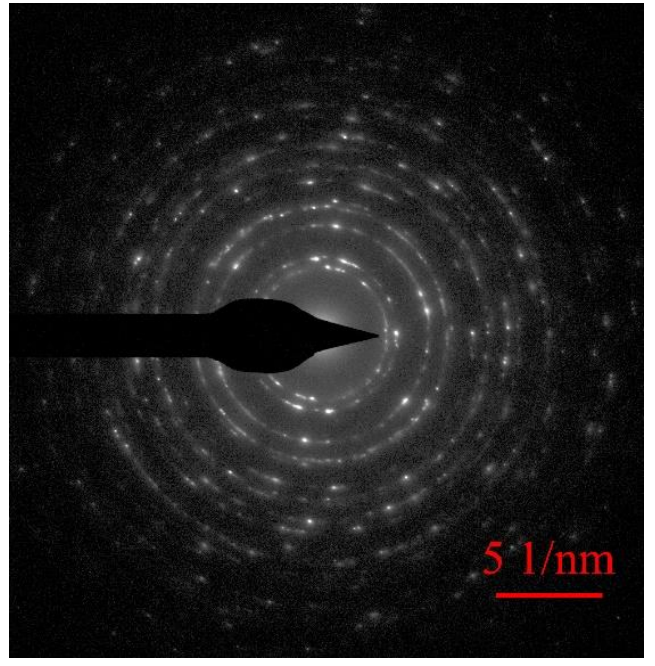

(a)

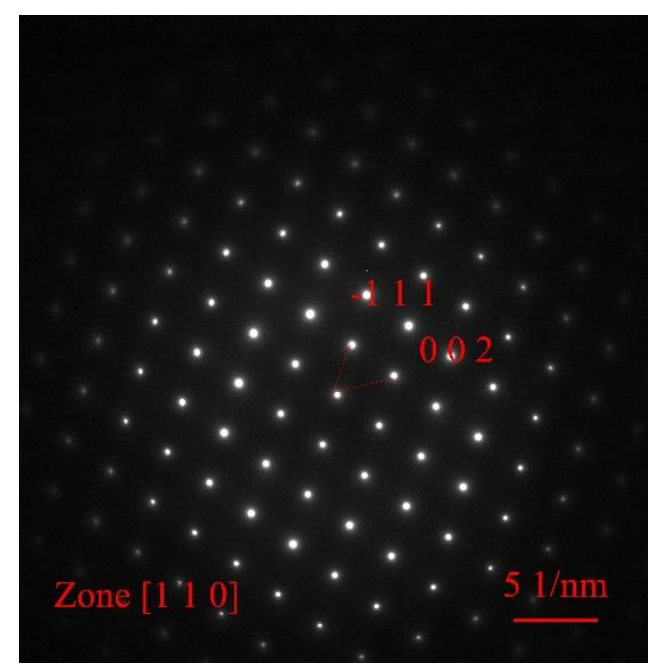

(b)

Figure 23. (a) Representative diffraction rings pattern from the nanocrystalline area of the lamella. (b) Isolated large grain present in the lamella. 


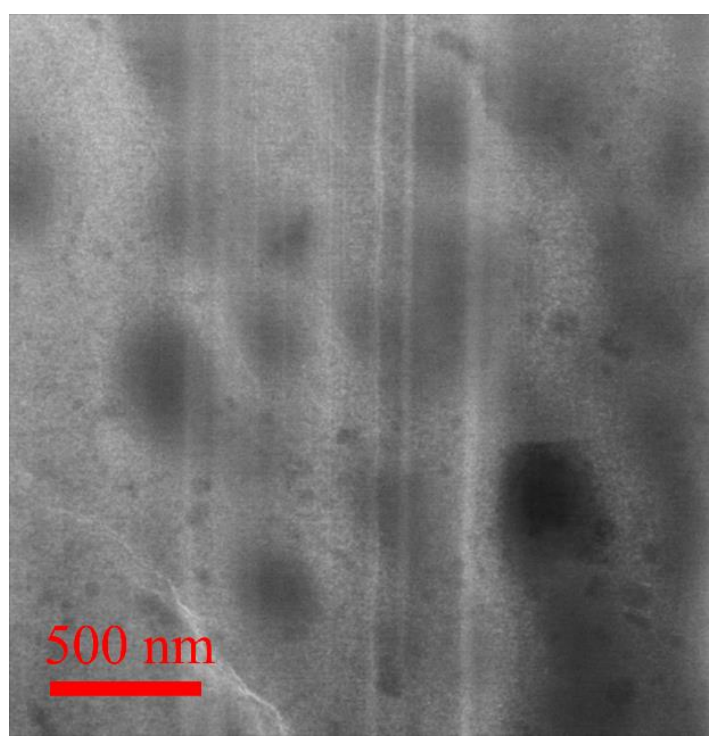

(a)

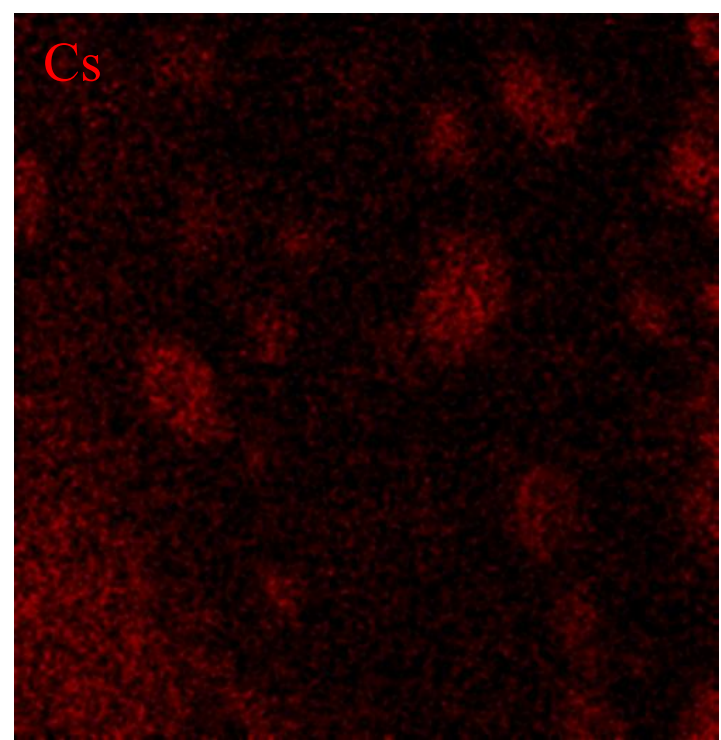

(b)

Figure 24. (a) Dark Field STEM image of the nanocrystalline domain. (b) EDS map showing Cs enrichment in some of the grains (map shows at\%).

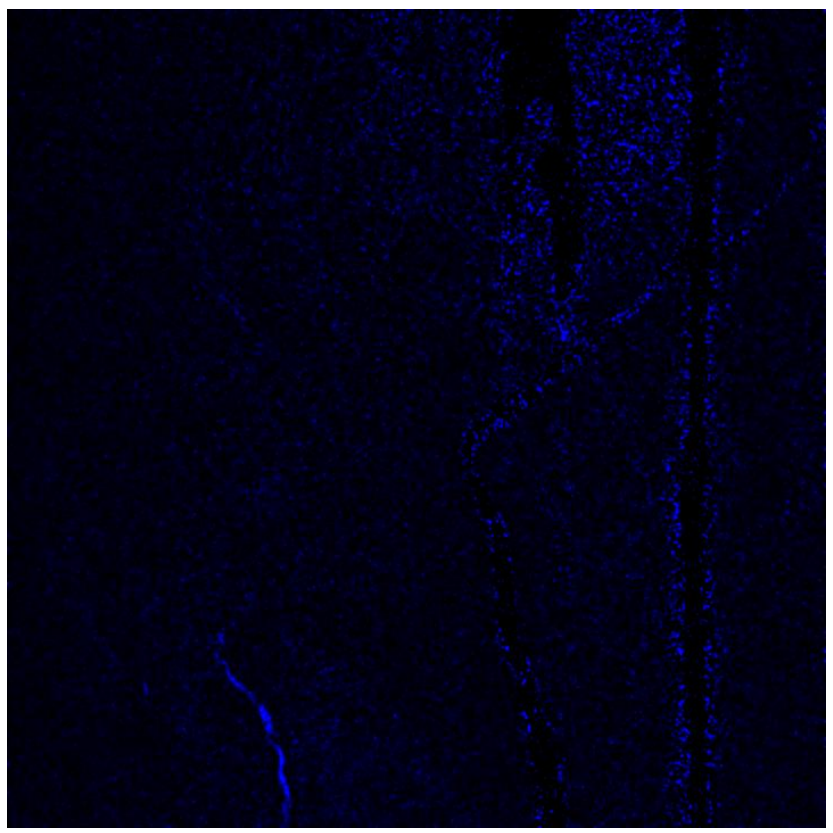

Figure 25. Atomic map of Te corresponding to the lamella area shown in Figure 19b.

The lamella extracted from area 4 showed that the chemical complexity increased further moving closer to the cladding inner surface. Only chemical data could be gathered. The atomic concentration maps of various elements are shown in the next figures. Figure 26a shows the gradient in the U-Pu concentration, which can be appreciated from the comparison of the two spectra from the indicated areas shown in Figure 27. Regarding the secondary phases, it can be seen that the precipitates are mainly composed of $\mathrm{Pd}$, which has separated from $\mathrm{Ru}$ and $\mathrm{Rh}$. No Mo was present in the metallic phases. The volatiles I, Te and Cs have condensed along the cracks, as seen in Figure 26f-h. Regarding the cladding components, the element which is present the most is $\mathrm{Cr}$, which has alloyed with $\mathrm{Ru}$ and $\mathrm{Rh}$. This behavior is quite unusual, as $\mathrm{Cr}$ is believed to be the first cladding element to oxidize in contact with the 
oxide fuel and form oxide species together with Cs $[25,26]$. Fe was detected in a few precipitates. Fe signal from the cracks is very weak, not always co-localized with either Te or I, which makes the quantitative assessment of the presence of iron tellurides or iodide challenging.

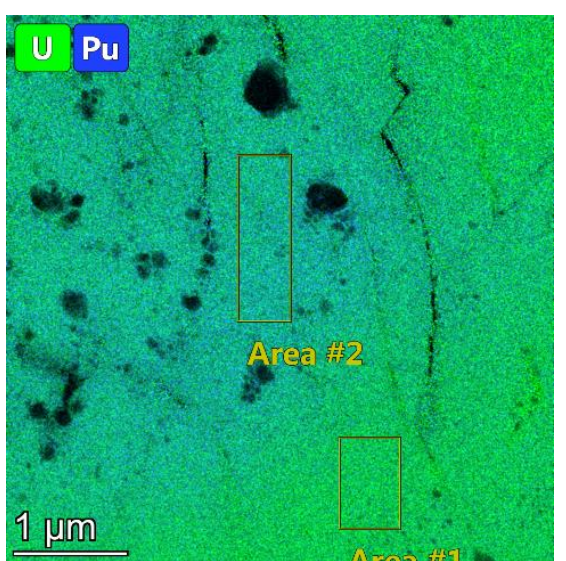

(a)

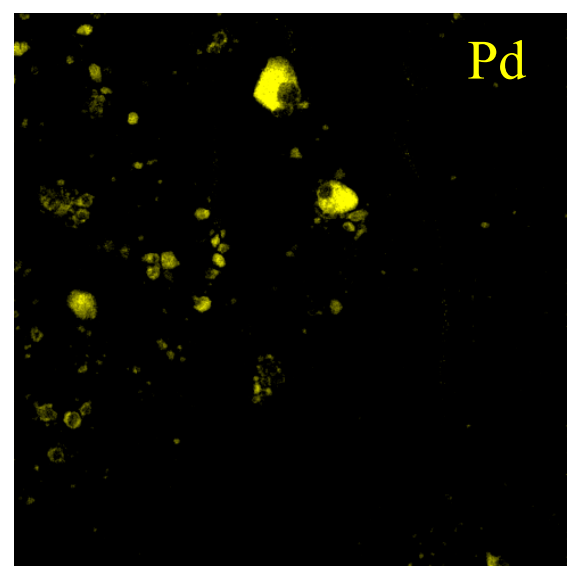

(b)

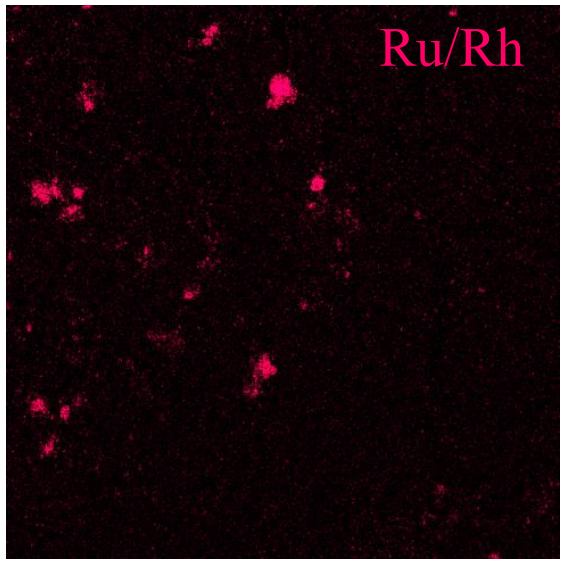

(c)

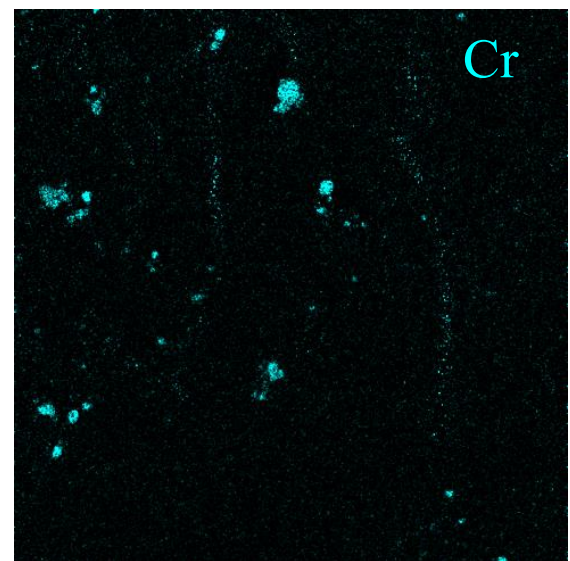

(d)

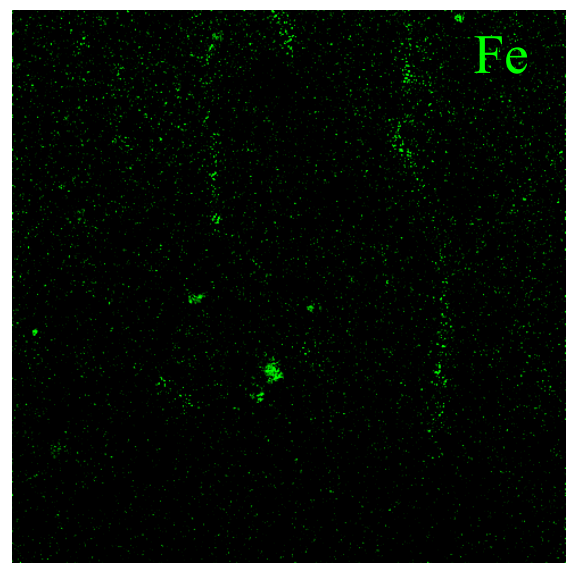

(e)

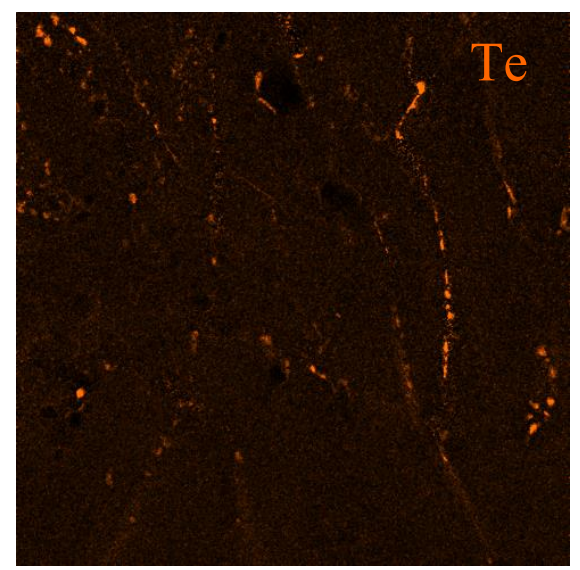

(f) 


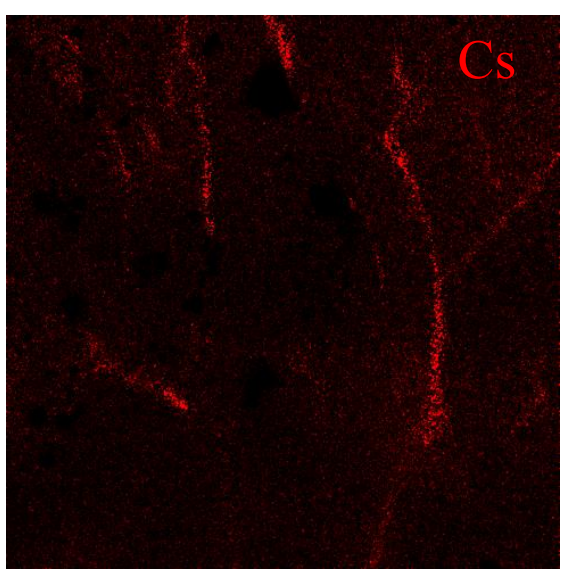

(g)

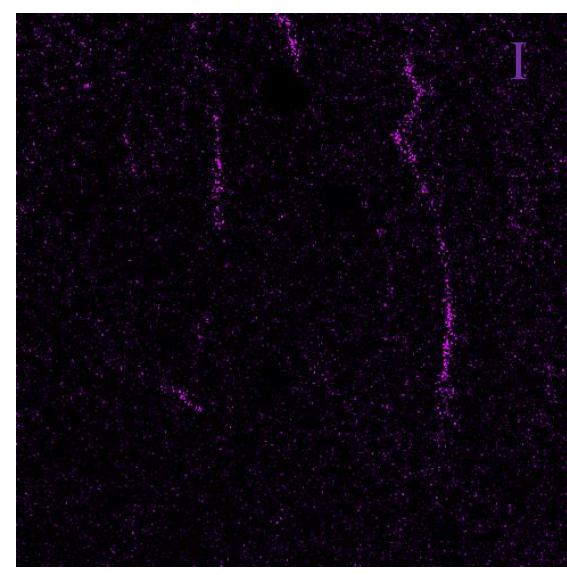

(h)

Figure 26. Atomic maps of the lamella from area 4.

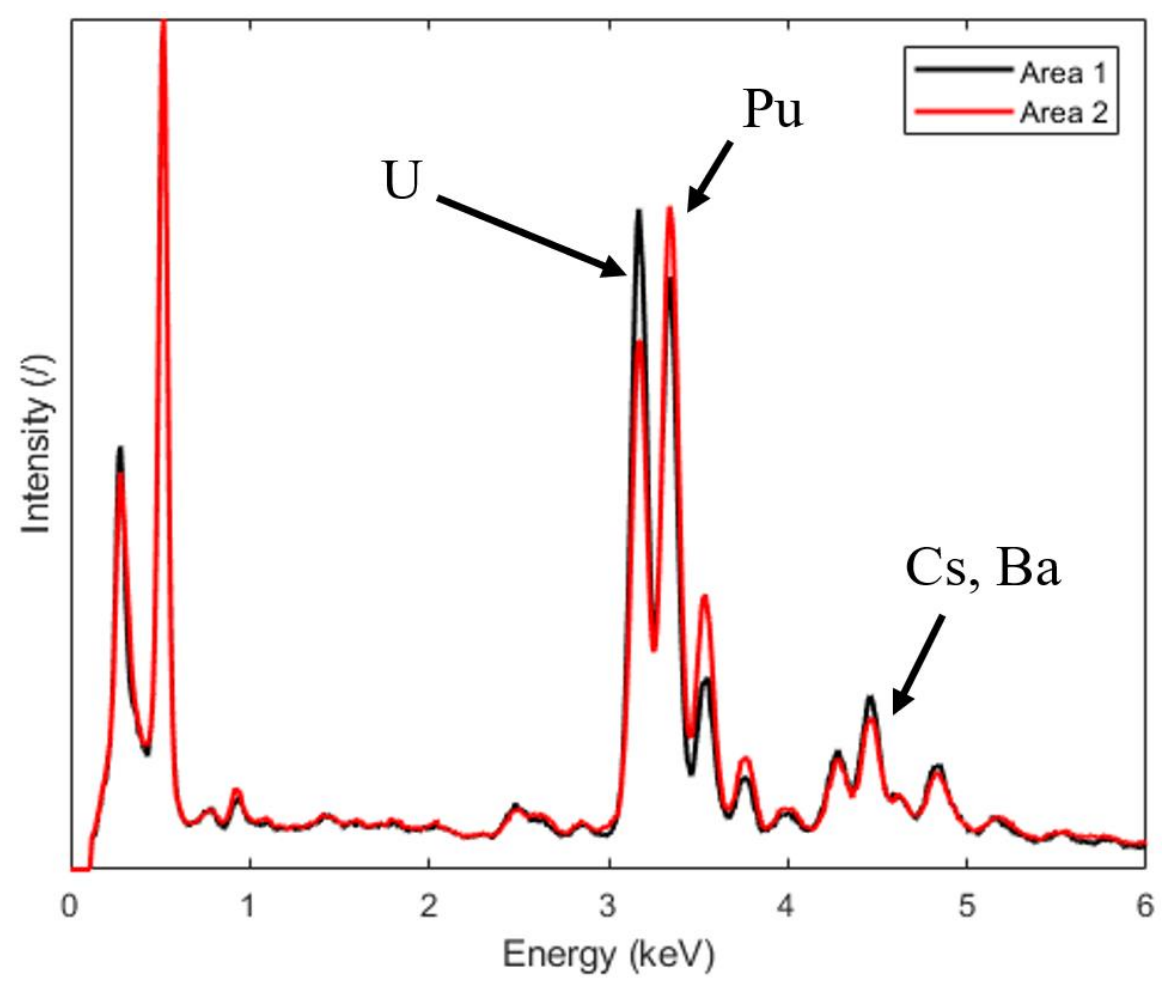

Figure 27. Spectra corresponding to area 1 and 2 in Figure 26a. The different enrichment in $\mathrm{U}$ and $\mathrm{Pu}$ can be appreciated by the relative height of the characteristic main peak.

\subsubsection{Sample 2}

\subsubsection{Fuel microstructure and fission products}

Similar investigations have been performed on the other sample from pin K06, which is shown in Figure 28. The microstructure shows similar features as in the previous sample, i.e., enlarged central hole, the columnar grain region and the equiaxed grain region. Images of the grains in the columnar grain region reveal a dense population of intergranular gas bubbles, some of which have connected forming tunnels (Figure 29b). Metallic precipitates decorate the surfaces of the bubbles. Various locations along the pellet periphery and inside the large cracks have been examined, as indicated in Figure 28 by the 
rectangular areas. Toward the crack tip in Area 1, an area covered by the grey phases was detected, radially located at the interface between the columnar grain and the equiaxed grain region. The secondary phases are shown in Figure 30. The phase contained $\mathrm{Ba}$ and its daughter $\mathrm{Ce}$. The presence of $\mathrm{Zr}$ could not be assessed, as the $\mathrm{Zr}$ edge corresponds to the Au M edge. Fuel microstructure in the equiaxed region and at the pellet's outermost areas has been analyzed and presented localized anomalies. In between area 1 and 2, a region with unconventional grain morphology was formed, as shown in Figure 31a. The region was characterized by a local enrichment in Pu content, as can be seen from the spectra in Figure $31 \mathrm{~b}$. Mixed oxide pellets were fabricated by mechanical blending of plutonia and urania powders [27]; hence, it is not surprising that occasional inhomogeneities could have occurred in the mixing. This could explain the Pu-enrichment in a localized island such as the one seen in Figure 31a. This being the case, a local increase in burnup could have occurred above the threshold of HBS formation, creating the subdivided grains seen in the island. EBSD maps have been acquired outside the island, and it can be seen that the subdivision remains local (Figure 32). The appearance of small grains has been observed in other regions, mainly inside large pores, such as those shown in Figure 33. The phenomenon remains confined in the cavities. Another area that showed onset of grain modification is visible in the fresh fracture surface in Figure 34, where the surface of some of the pre-existing grains is presenting signs of morphology change (top right of the figure). The surface corresponds to area 2 in Figure 28 where a piece of fuel broke off before the SEM analyses.

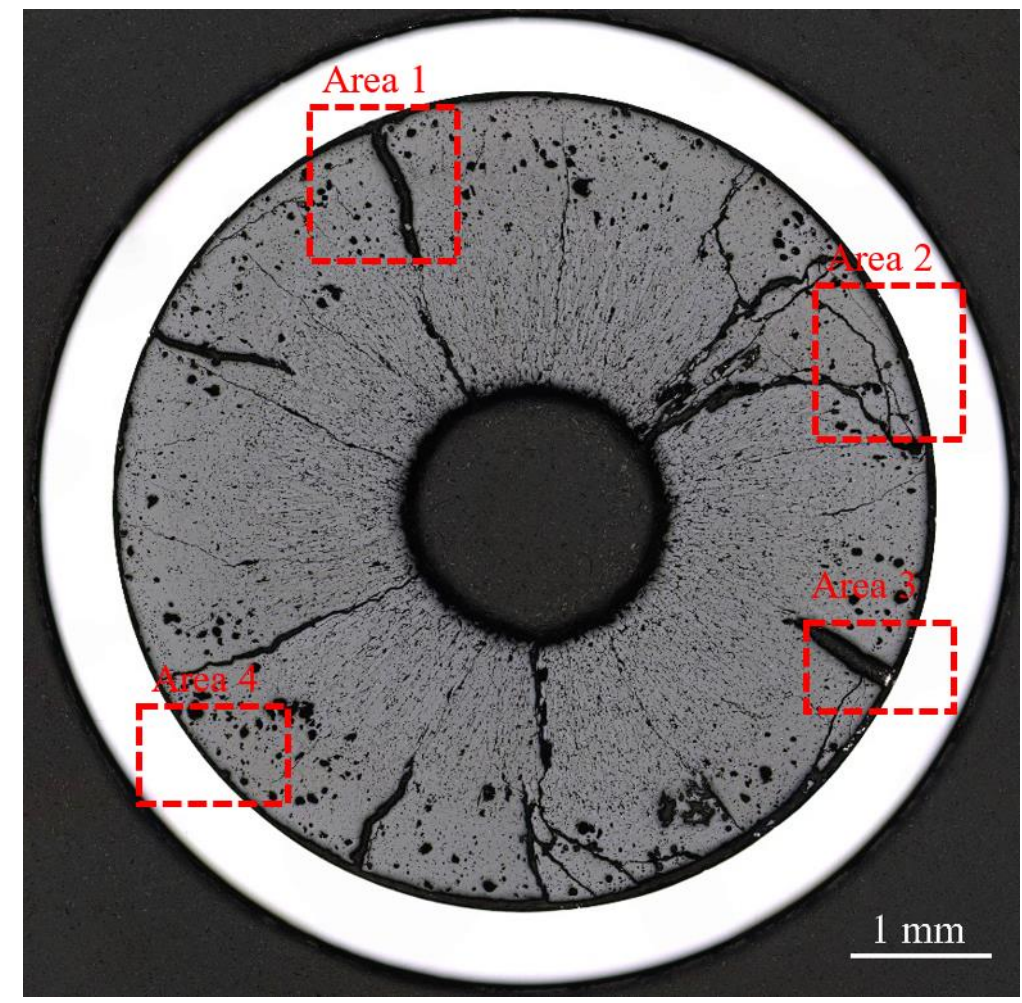

Figure 28. Overview of sample 2. 


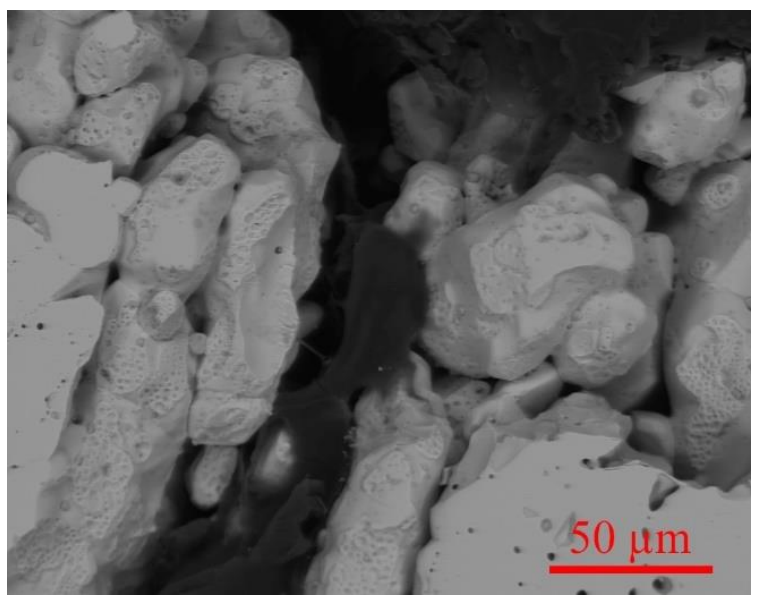

(a)

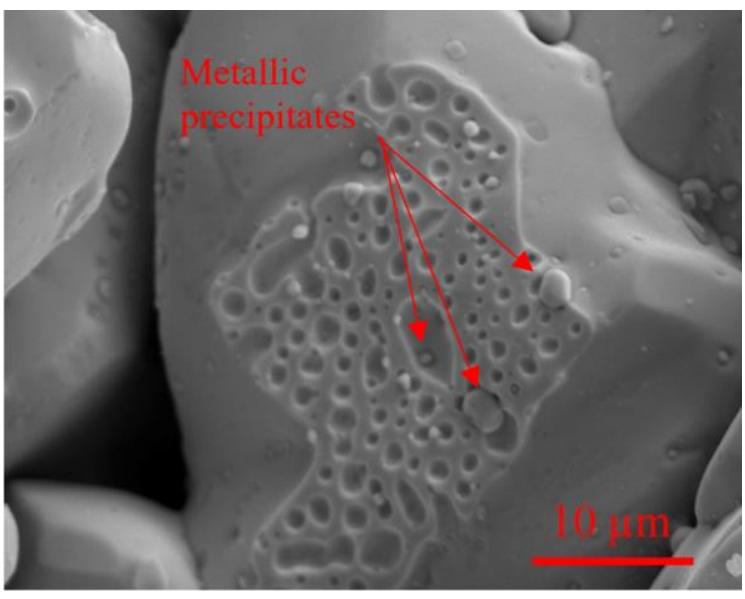

(b)

Figure 29. (a) BSE image of the grains close to the central hole. (b) High magnification of one of the grains showing the grain surface decorated with gas bubbles and metallic precipitates.

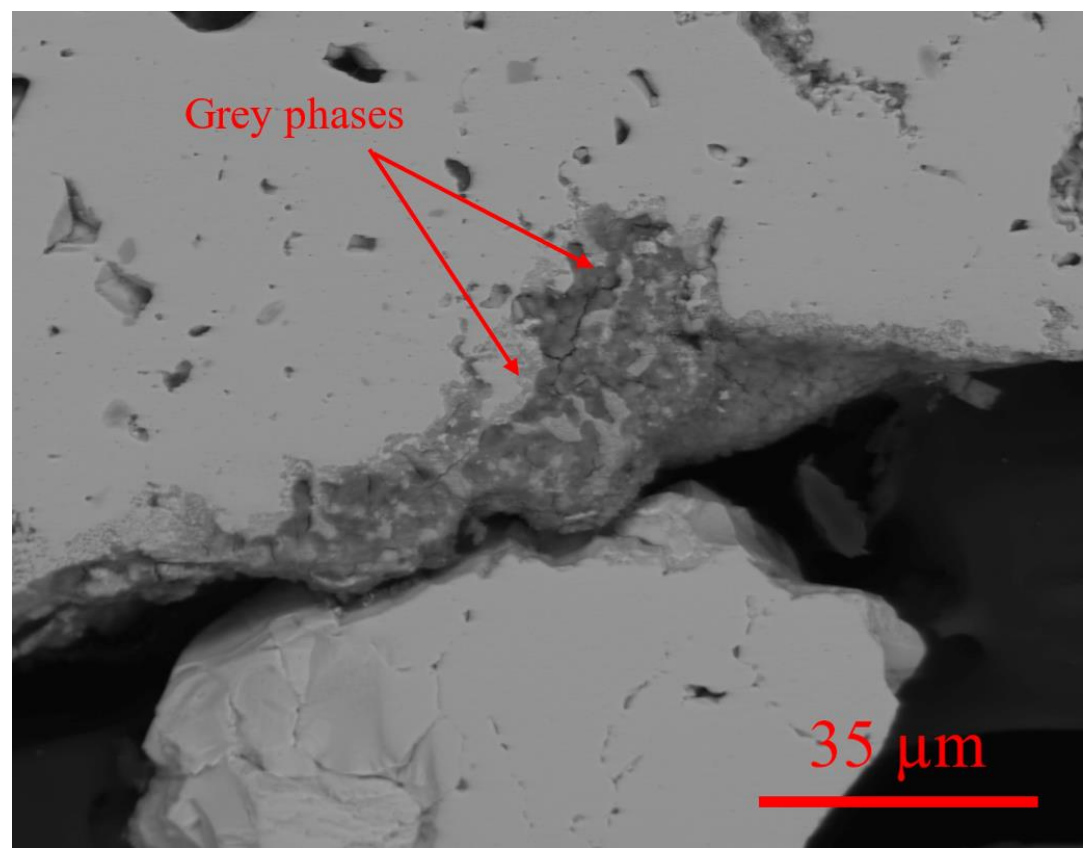

Figure 30. Accumulation of grey phases along a large crack surface. 


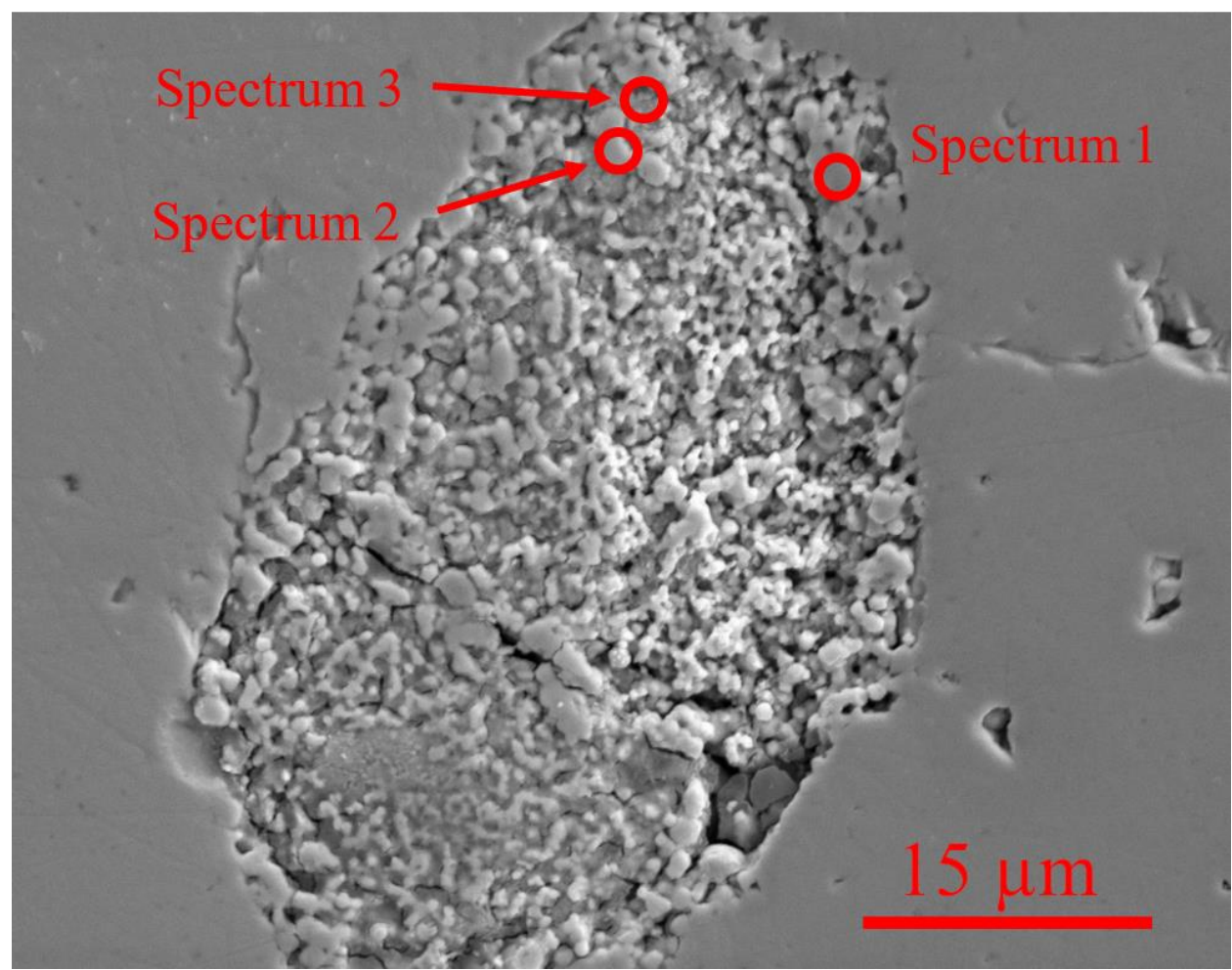

(a)

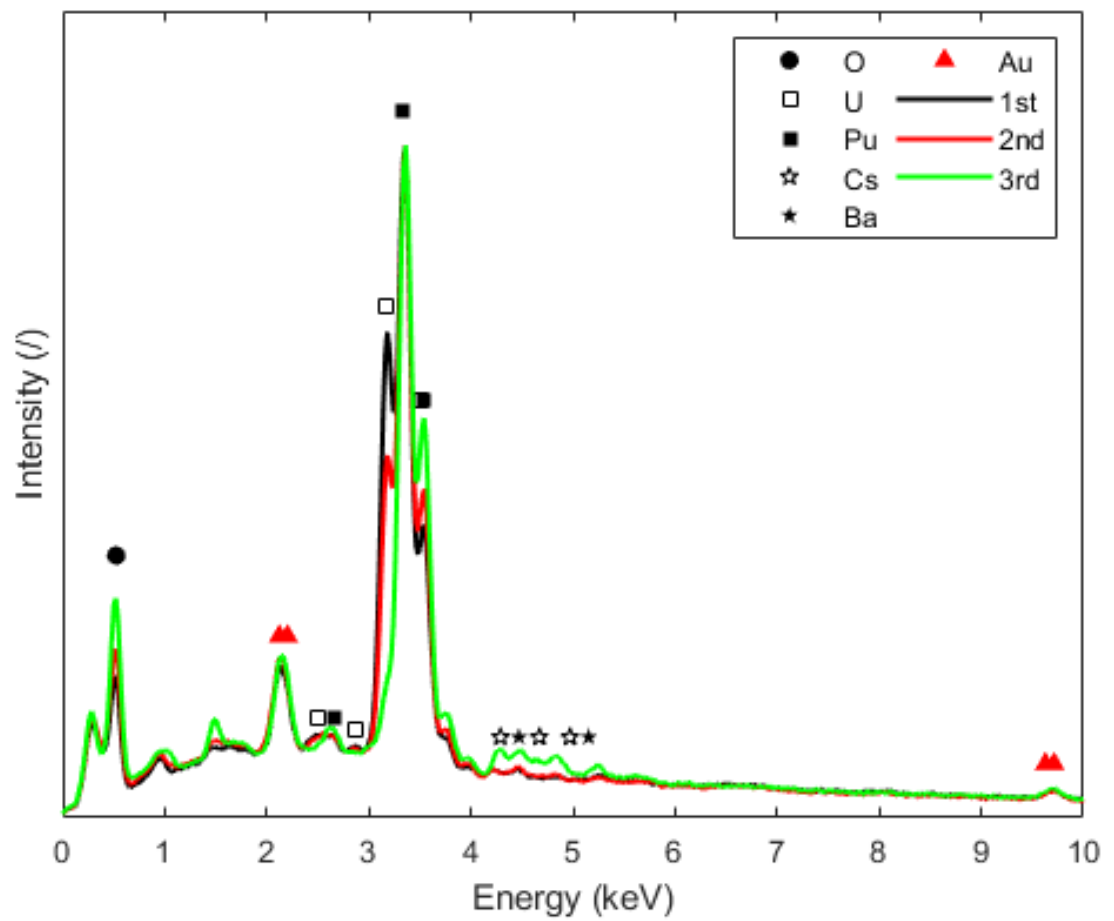

(b)

Figure 31. (a) Region showing unconventional grain morphology within the equiaxed grain area. (b) Spectra corresponding to the marked regions in (a). 

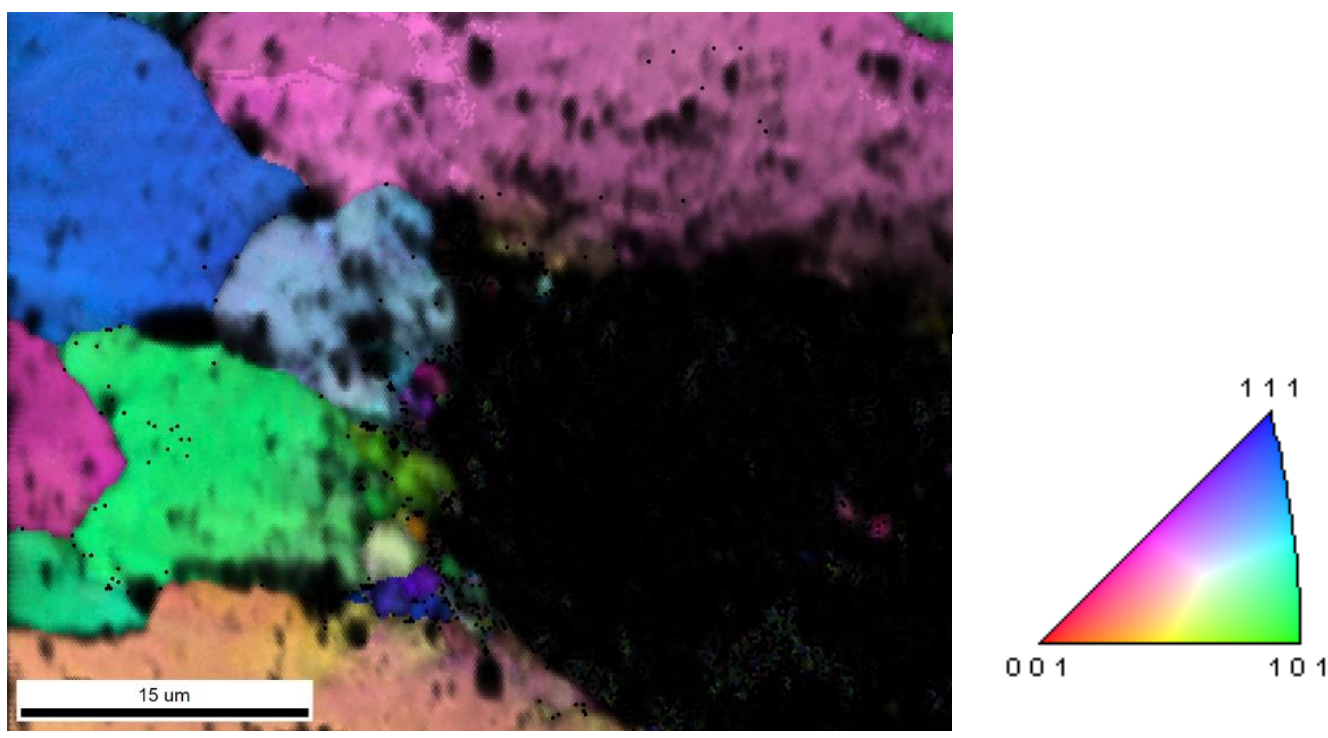

Figure 32. IPF and IQ map outside the restructured pool from Figure 31a.

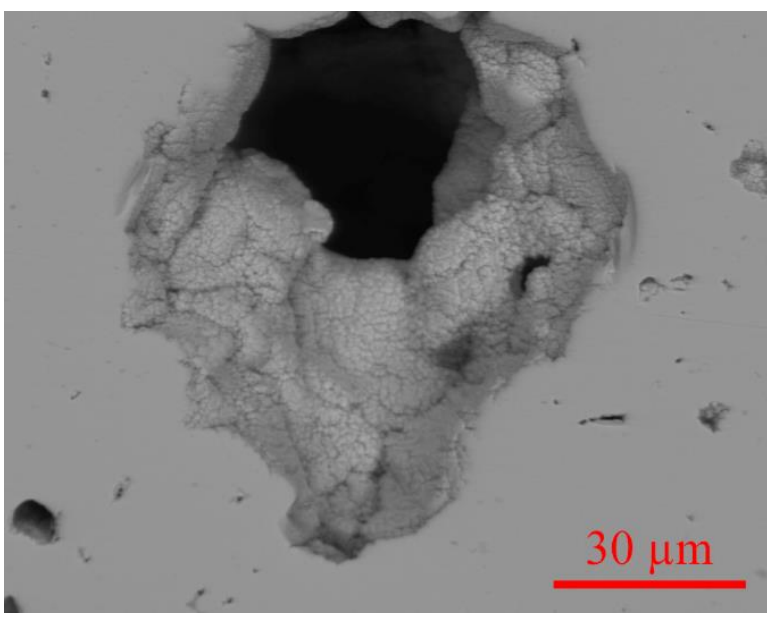

(a)

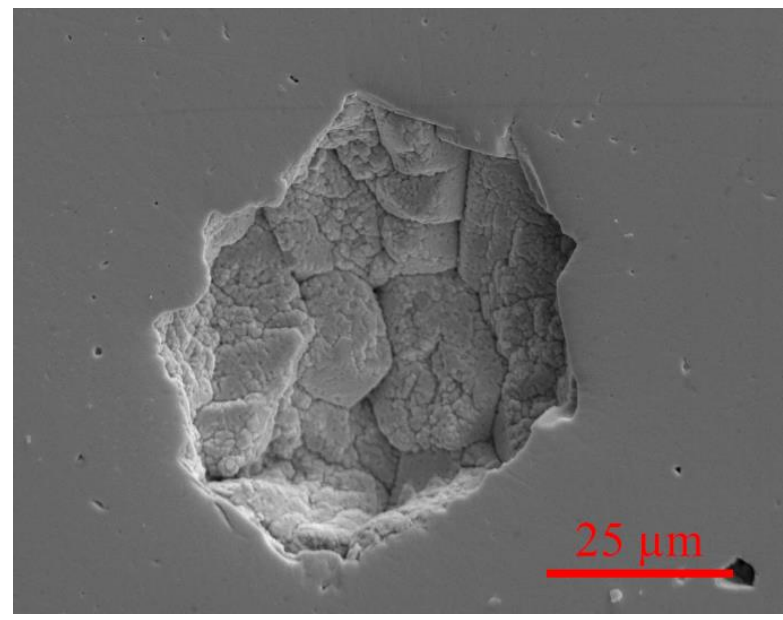

(b)

Figure 33. (a)-(b) Examples of hole surface showing grain subdivision. 


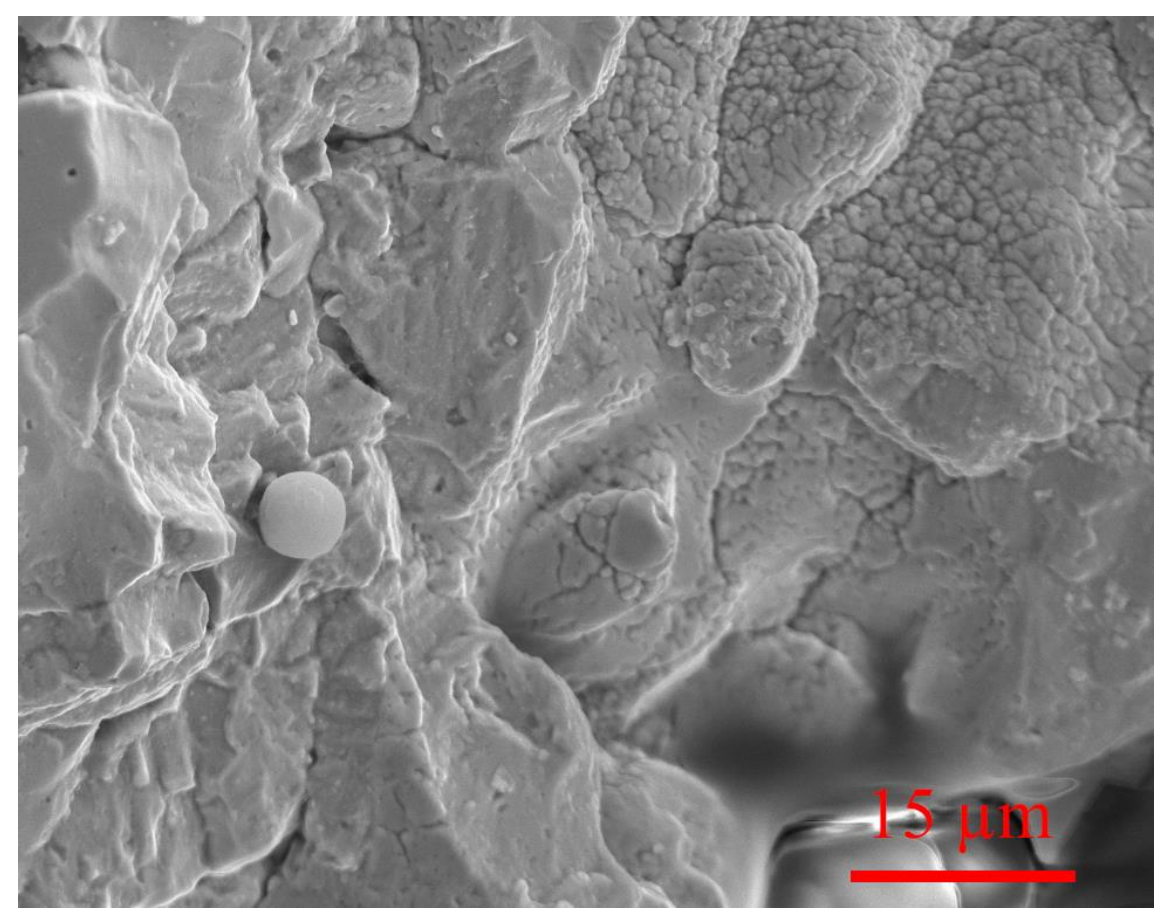

Figure 34. Missing fuel piece from area 2 showing onset of grain subdivision on the surface of preexisting grains.

\subsubsection{Fuel-cladding chemical interaction}

The precipitation of fission products and the interaction with the cladding was more extensive than in the previous sample, consistent with the higher cladding temperatures that are known to play a significant role in the extension of the FCCI. Part of the cladding showed onset of intergranular corrosion, as can be seen in Figure 35. Other azimuthal direction showed that the cladding inner surface was covered by a deposit mainly composed of Pd, which can be seen in the light color attached to the cladding in Figure 36. The "liner" thickness remains below $5 \mu \mathrm{m}$, but localized thickness increase of the attacked zone was observed in various locations, e.g., in Figure 37, Figure 38, Figure 39 and Figure 40. The composition of the corroded zones varies, being either metallic (mainly where Pd is present, occasionally $\mathrm{Ag}$ too) or an oxide (for example, see Figure 37h). In some cases, it contains fuel elements, while in others, it does not. The most prominent fission products are $\mathrm{Pd}, \mathrm{Cs}$, which are always detected. Te is often also present in different amount, while I typically occurs as in traces. Cr seems to form oxides and compounds with local presence of Cs (see Figure 37b, d and h) or Te, likely alloyed with I. Further TEM analyses reported below showed that, in the majority of the cases, $\mathrm{Cr}$ remains in metallic phase. Where the thickness of the corroded layer was beyond $10 \mu \mathrm{m}$, as in Figure 40, regions with different compositions were present, as can be seen from the spectra in Figure 40b. In this area, additional analyses have been performed by TEM, extracting a lamella as indicated by the blue rectangle in Figure 40a. 


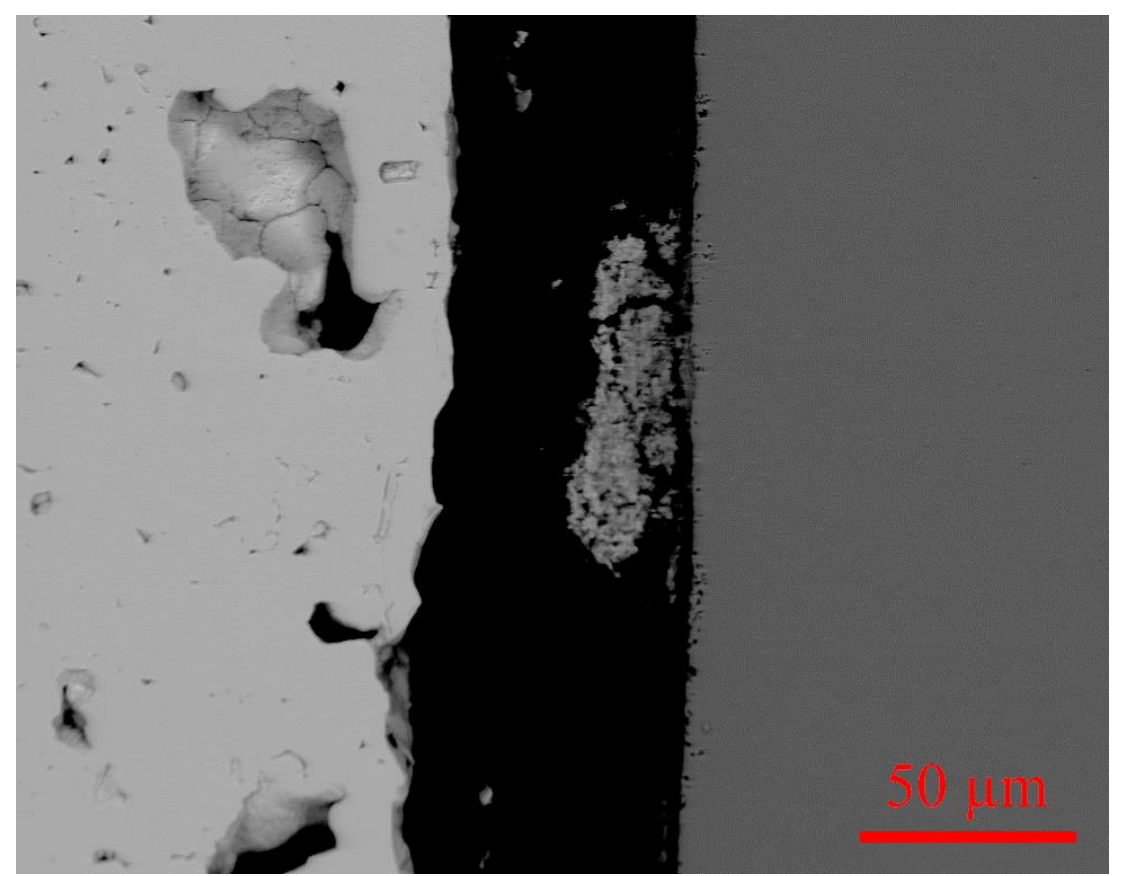

Figure 35. Onset of intergranular corrosion at the inner surface of the cladding surface.

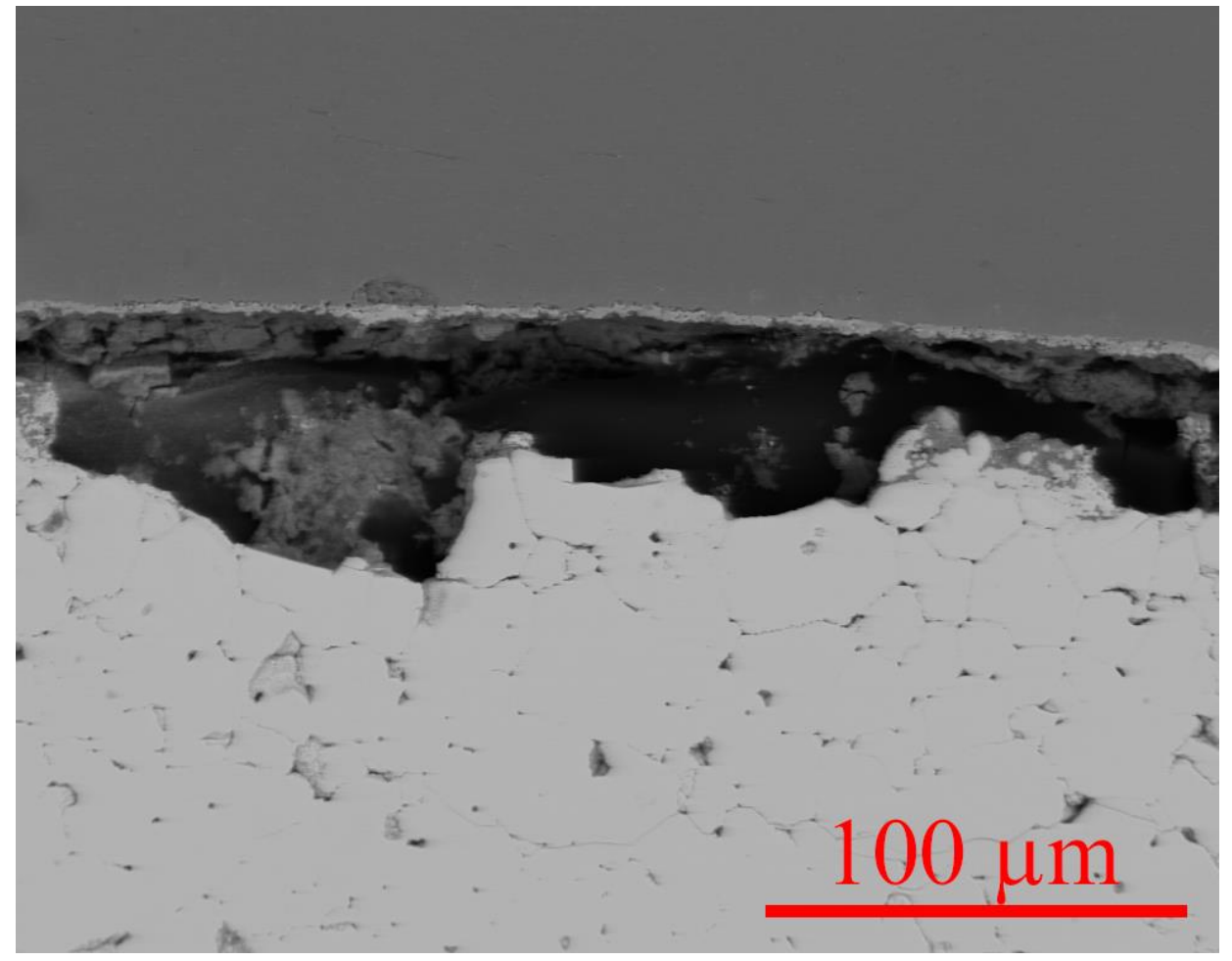

Figure 36. BSE image showing the Pd liner formation on the inner cladding surface. 


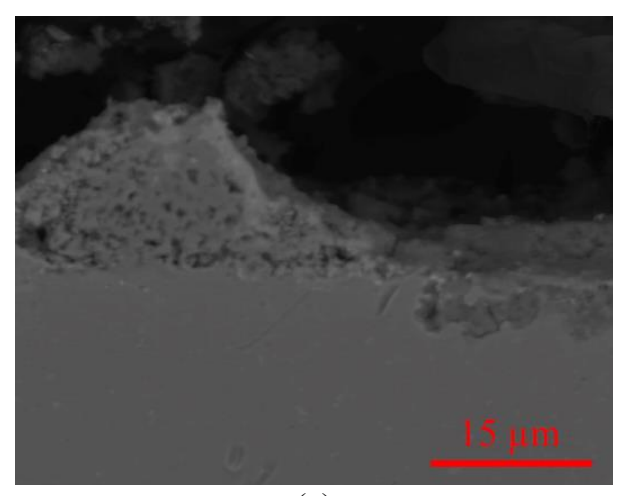

(a)

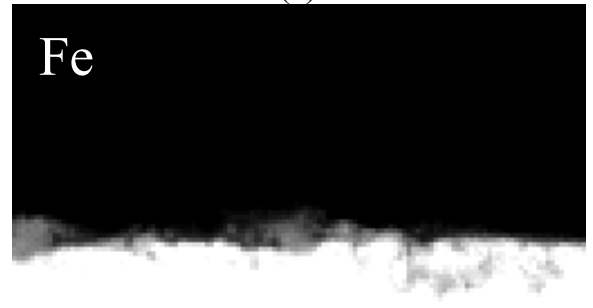

(c)

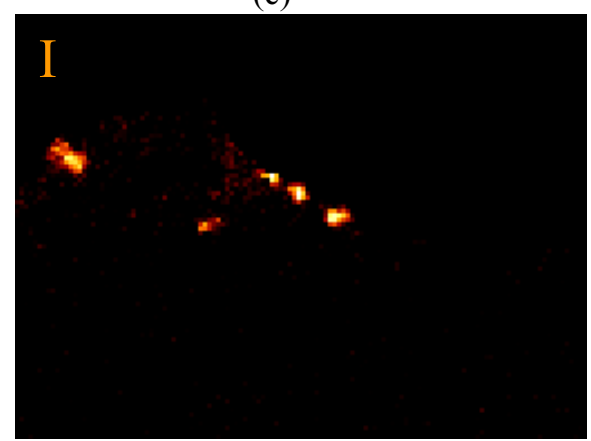

(e)

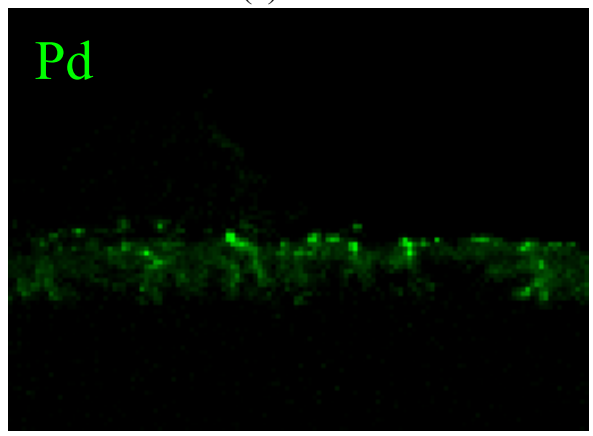

(g)

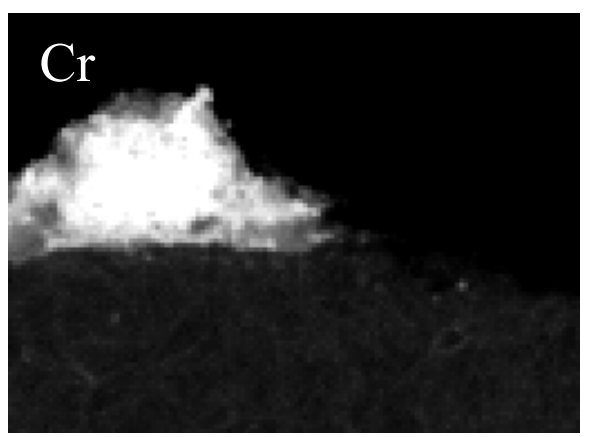

(b)

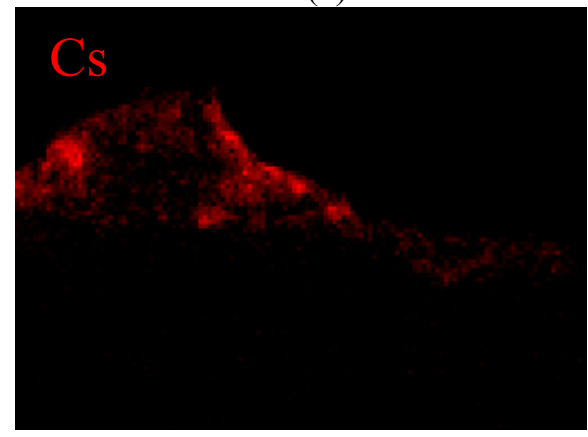

(d)

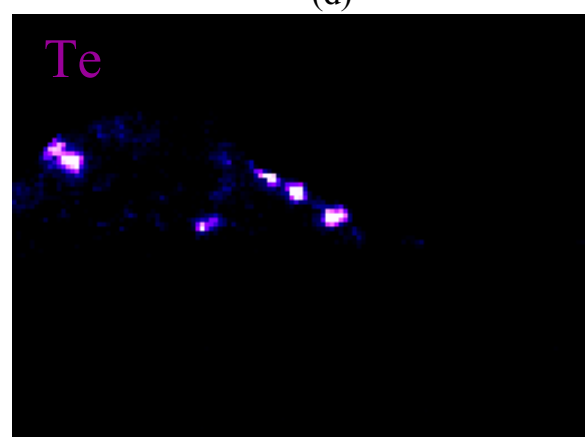

(f)

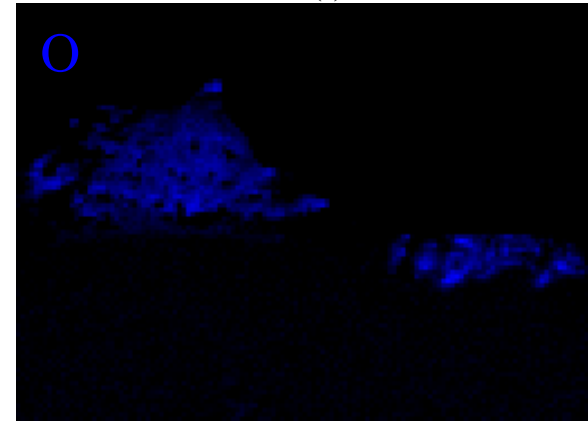

(h)

Figure 37. (a) BSE image of FCCI. (b)-(h) Chemical mapping of fission products and major cladding components. No $\mathrm{U}$ and $\mathrm{Pu}$ were present in the corrosion island. 


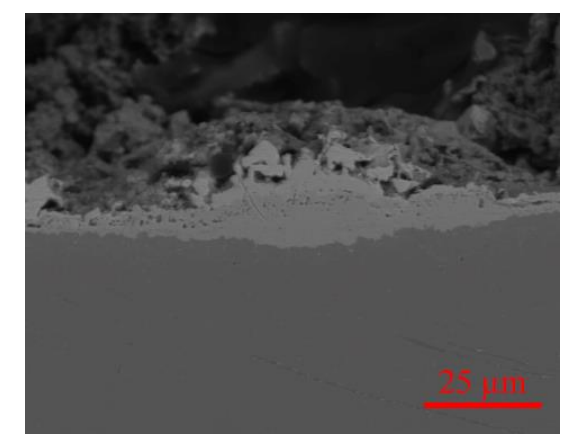

(a)

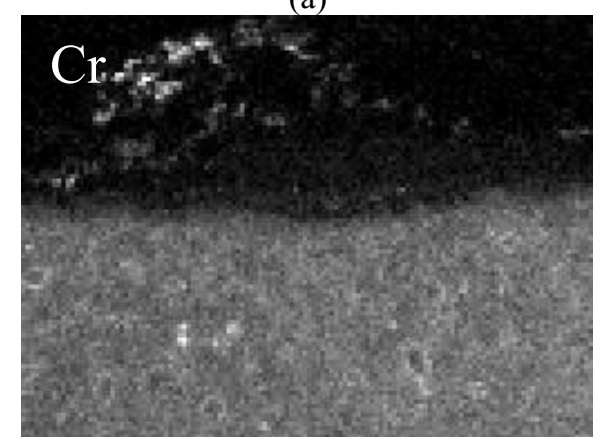

(c)

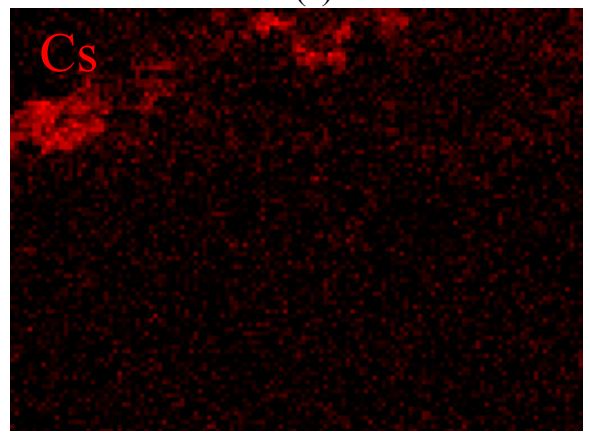

(e)

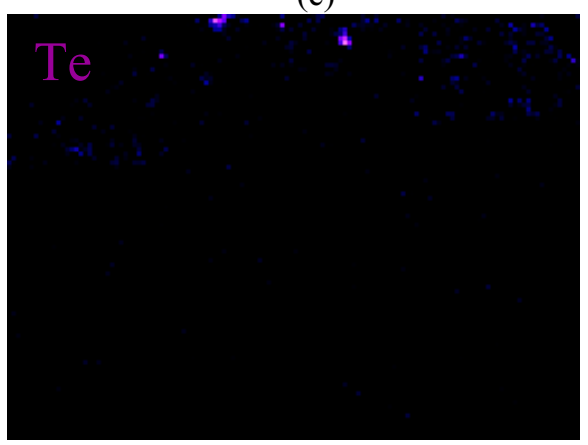

(g)

Figure 38. (a) BSE image of FCCI. (b)-(f) Chemical mapping of fuel, fission products and major cladding components. Maps in (c) and (e) correspond to WDS signal, whereas the others are EDS maps. 


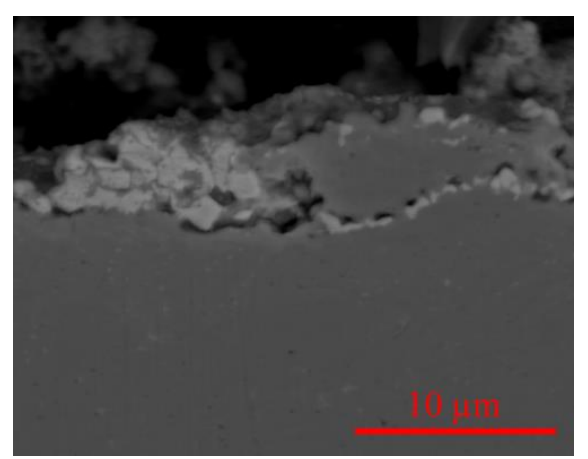

(a)

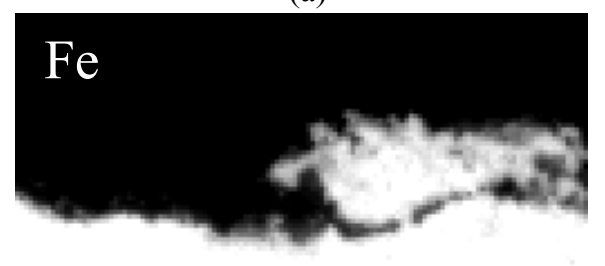

(c)

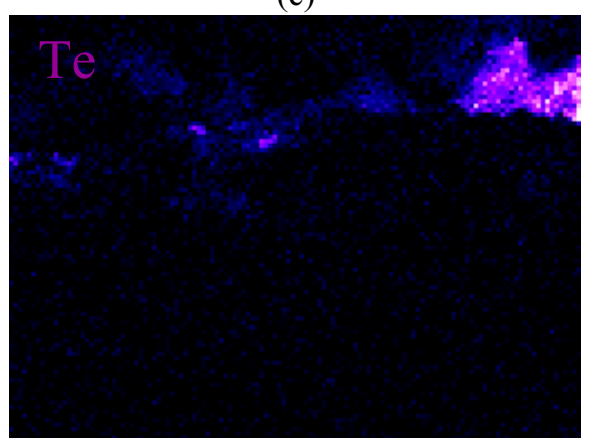

(e)

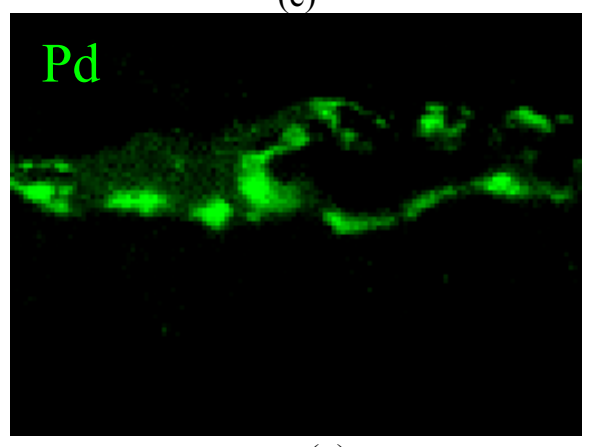

(g)

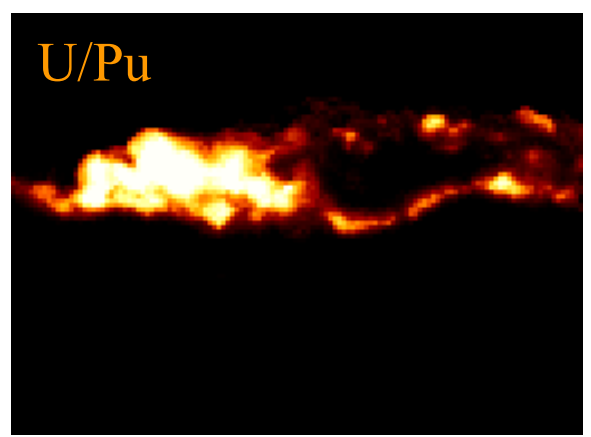

(b)

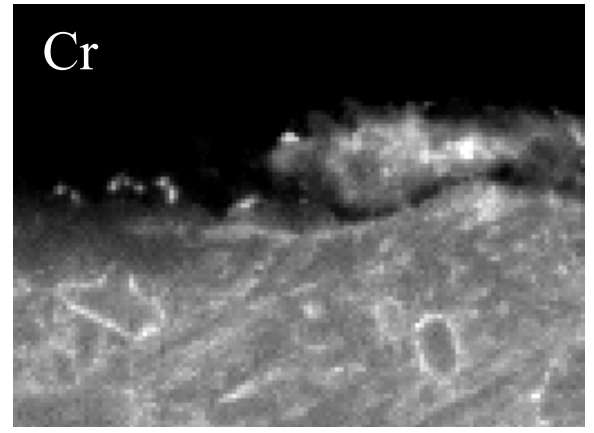

(d)

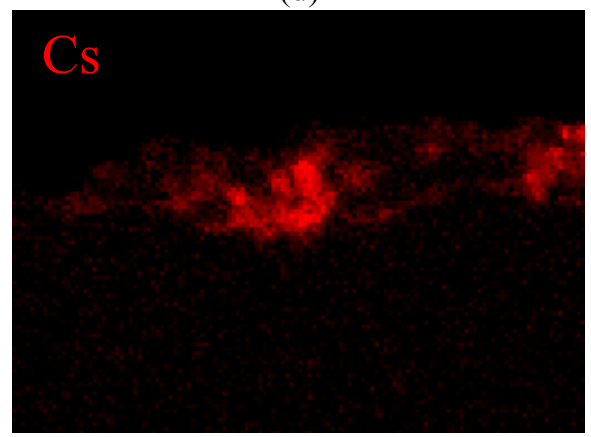

(f)

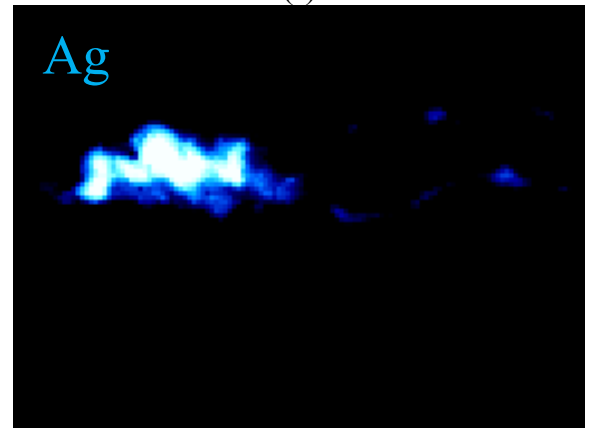

(h)

Figure 39. (a) BSE image of FCCI. (b)-(h) Chemical mapping of fuel, fission products and major cladding components. Maps in (e) and (g) correspond to WDS signal, whereas the others are EDS maps. 


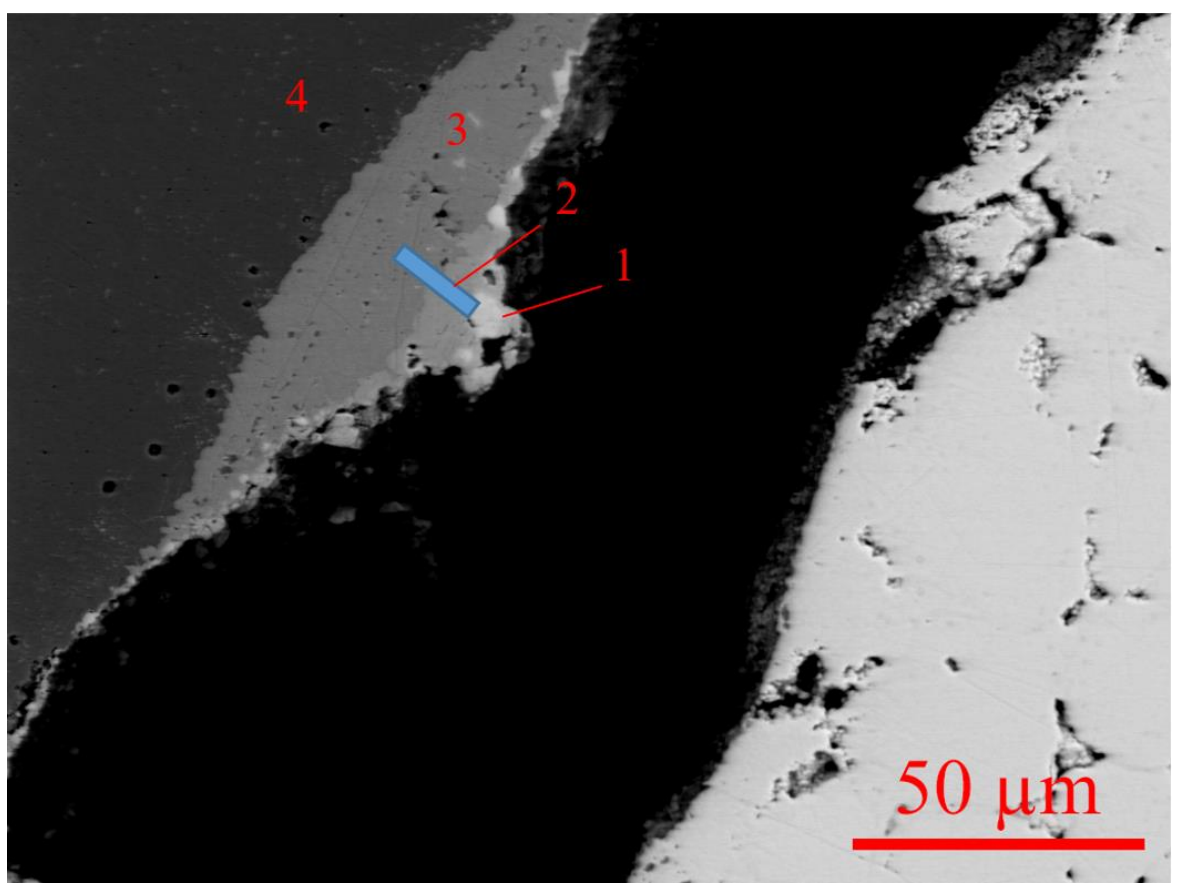

(a)

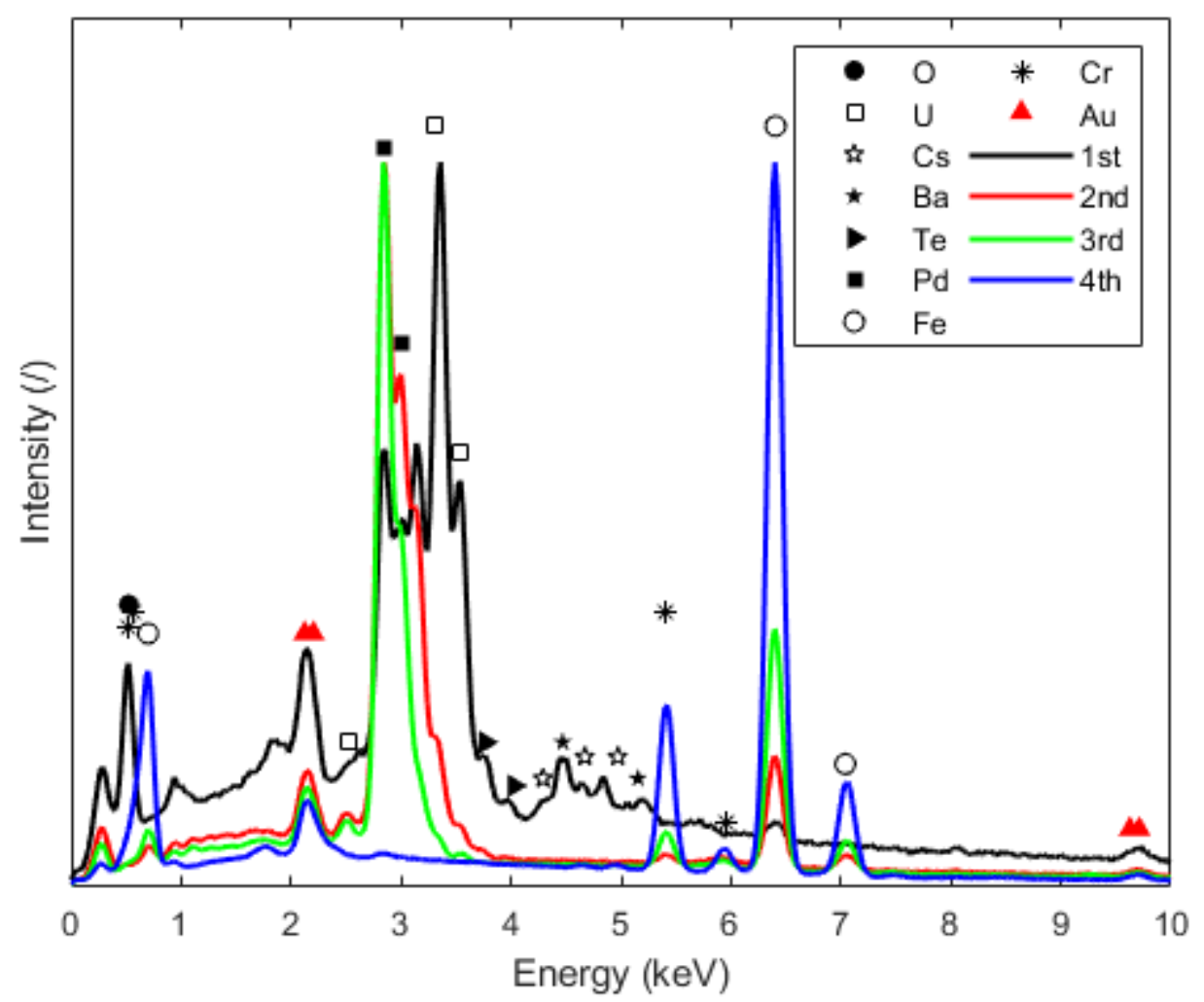

(b)

Figure 40. (a) Region with increased FCCI thickness, showing existence of four regions with different compositions showed in (b). 
The overview of the composition of the lamella is shown in Figure 41. The left side of the lamella has the typical composition and structure of HT-9, with Pd intergranular inclusions (see Figure 42b). Moving towards the right of the lamella, the composition changes, showing a marked enrichment in $\mathrm{Cr}$, as highlighted by both the map and the line scan in Figure 42c and d, respectively. In this Cr-enriched layer, the Cr-enrichment varied from point to point, going from a minimum of 40 at $\%$ to almost 80 at $\%$. In Figure 43 SAED patterns from the Cr-rich area are shown. The best fit was given by the tetragonal structure of $\sigma$-FeCr (space group $\mathrm{P} 42 / \mathrm{mnm}, \mathrm{a}=8.800 \AA, \mathrm{c}=4.544 \AA$ ) [28]. Although this phase is predicted to be stable only above $600^{\circ} \mathrm{C}$, it has already been shown that the formation of this phase was enhanced by neutron irradiation, and the temperature range extended downwards to $550^{\circ} \mathrm{C}$ in austenitic AISI 316 [29]. The observation of phases outside the predicted stable range after irradiation is not uncommon in highly irradiated materials.

Within the Cr-rich region, nanocrystalline inclusions are visible, some of which are Cs- and Ba-rich (Figure 44b), while others are enriched in Te and show presence of I (Figure 44d). Unfortunately, the sample broke before the atomic concentration in the precipitates could be measured extensively, and only a local spectrum corresponding to the area circled in Figure 44a could be obtained. The spectrum and the measured composition are reported in Figure 45 and Table 2, respectively. The nanocrystalline phase is an oxide with multiple fission products included, as well as minor quantities of $\mathrm{U}$ and $\mathrm{Pu}$ and cladding components. The major constituents, excluding oxygen, are $\mathrm{Cr}$ and $\mathrm{Cs}$ (together with its daughter $\mathrm{Ba}$ ), suggesting the formation of $\mathrm{Cs}_{\mathrm{x}} \mathrm{CrO}_{4}$. Notable content of $\mathrm{Y}$ is visible. Only Y-89 is a stable isotope, which is likely to be the decay product of $\mathrm{Kr}-89$, a gaseous product with high cumulative yield for $\mathrm{Pu}-239$ (0.0105 from JEFF-3.3 library). Hence, the presence of Y could be explained if release and trapping of $\mathrm{Kr}-89$ had occurred in the precipitates. The morphology and distribution of these inclusions seems to indicate that a molten liquid containing several fission products, but mainly $\mathrm{Cs}$ and $\mathrm{Te}$, were in contact with the cladding, which has then crystallized upon cooling. The $\mathrm{Cr}$ has diffused and accumulated towards the inner surface, but it has not yet been oxidized completely. These two observations would point towards an environmental domain of attack compatible with a Fission Product-assisted Liquid Metal Embrittlement/Fuel Adjacency Effect (FPLME/FAE), in line with the corrosion attack mode schematic proposed by Adamson and Aitken [13], as indicated in Figure 46. 


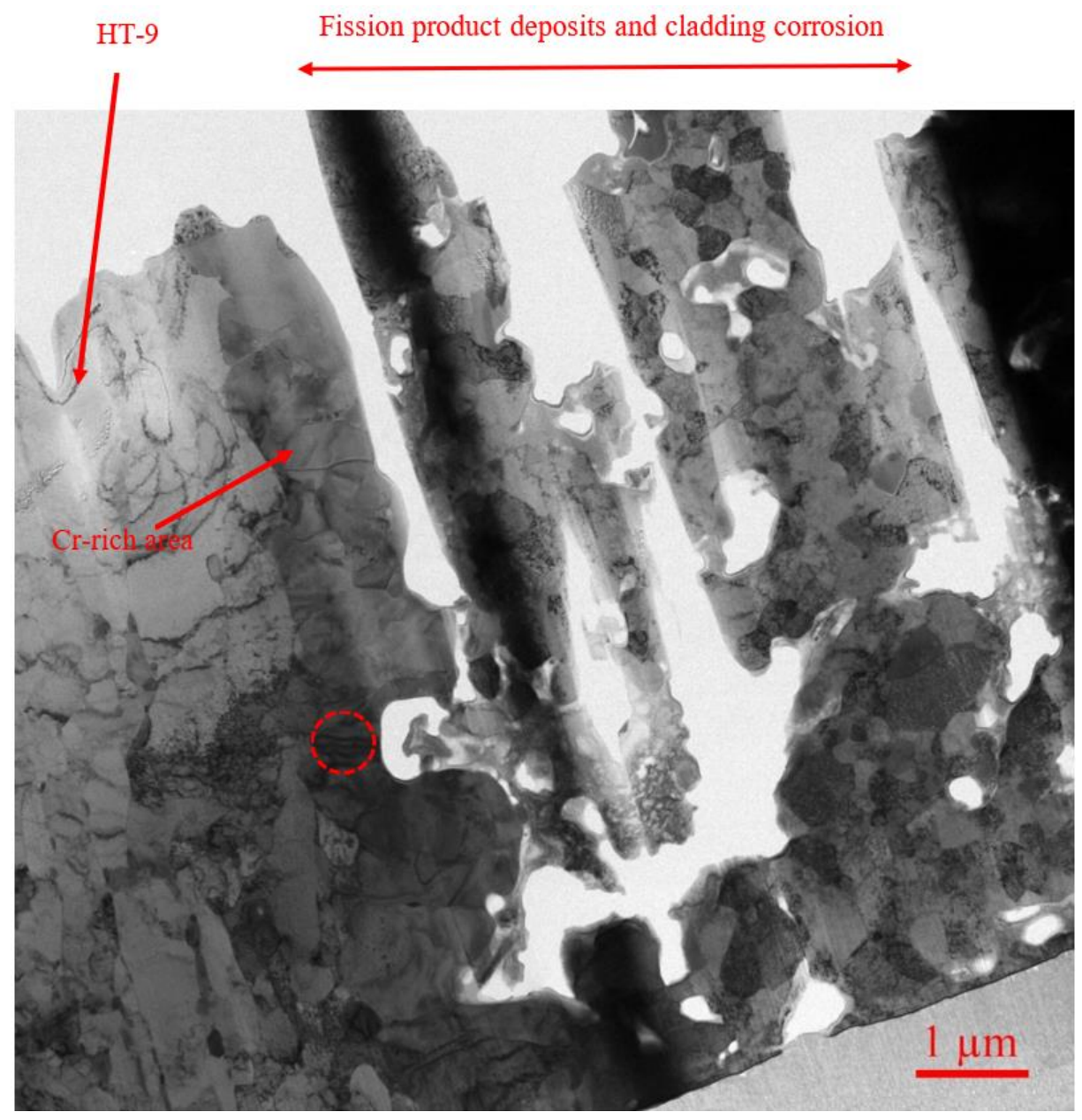

Figure 41. Overview of the lamella extracted between zone 2 and 3 in Figure 40a. The red circle indicates the location of the SAED patterns in Figure 43. 


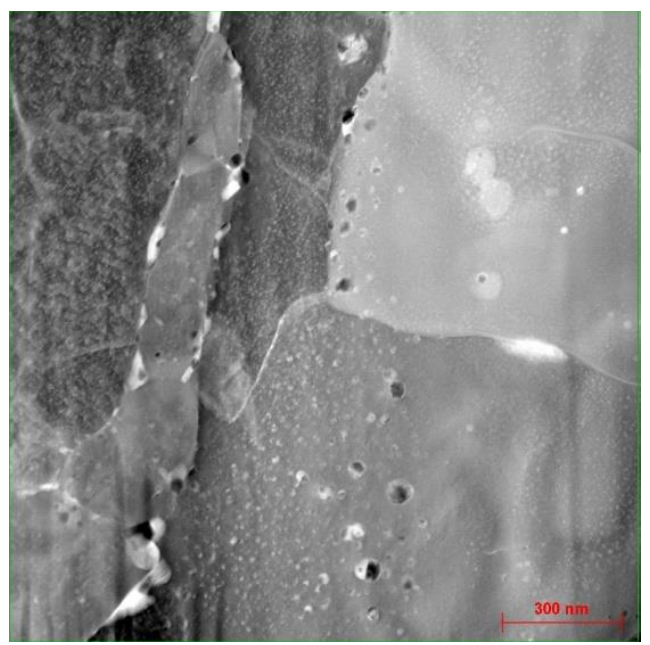

(a)

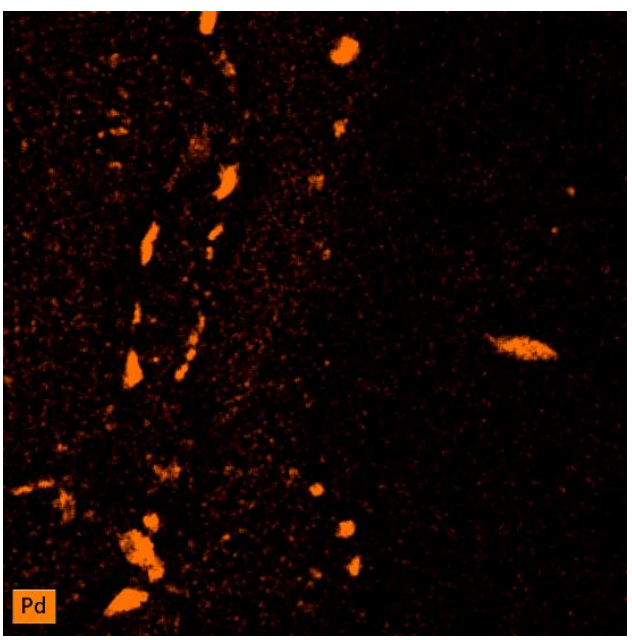

(b)

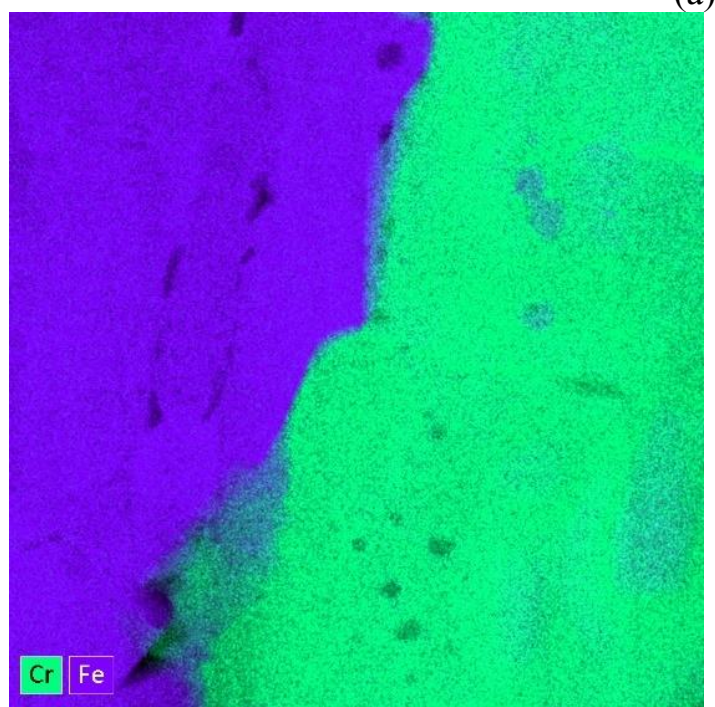

(c)

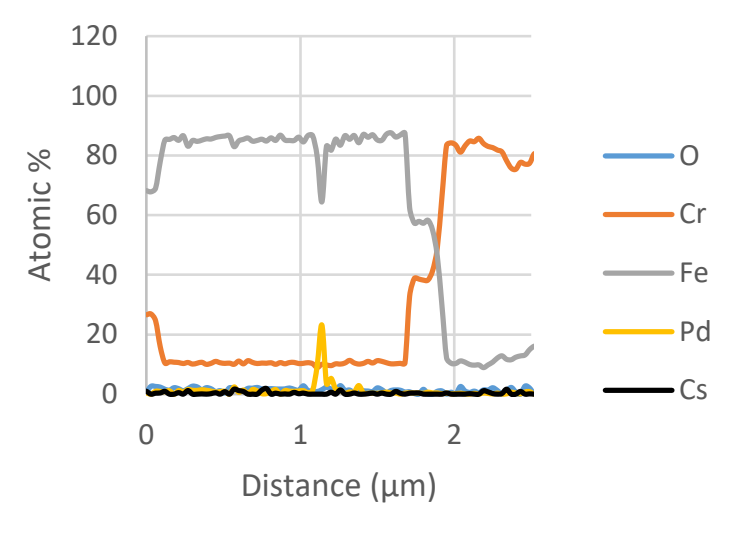

(d)

Figure 42. (a) HAADF of the interface between the HT-9 and the Cr-rich region. (b), (c) Qualitative EDS maps showing precipitation of $\mathrm{Pd}$ along grain boundaries and the $\mathrm{Cr}$-rich interface. (d) Normalized element concentration across the two phases. 


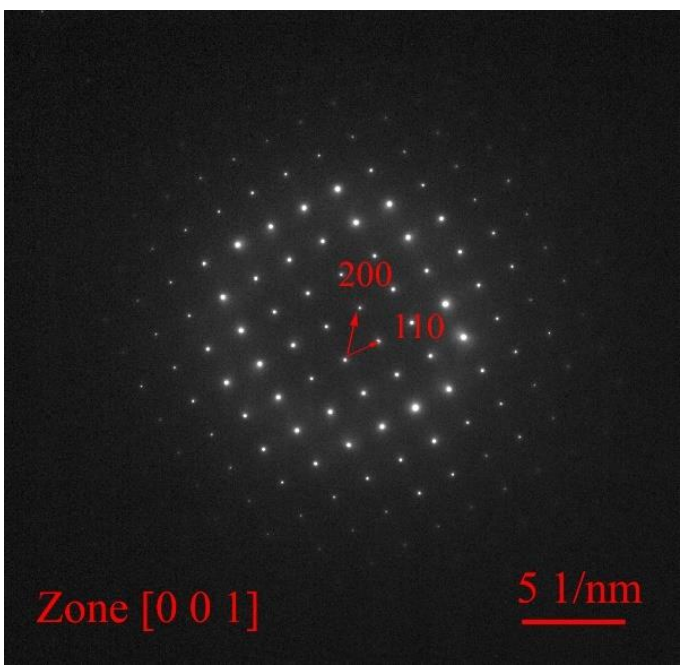

(a)

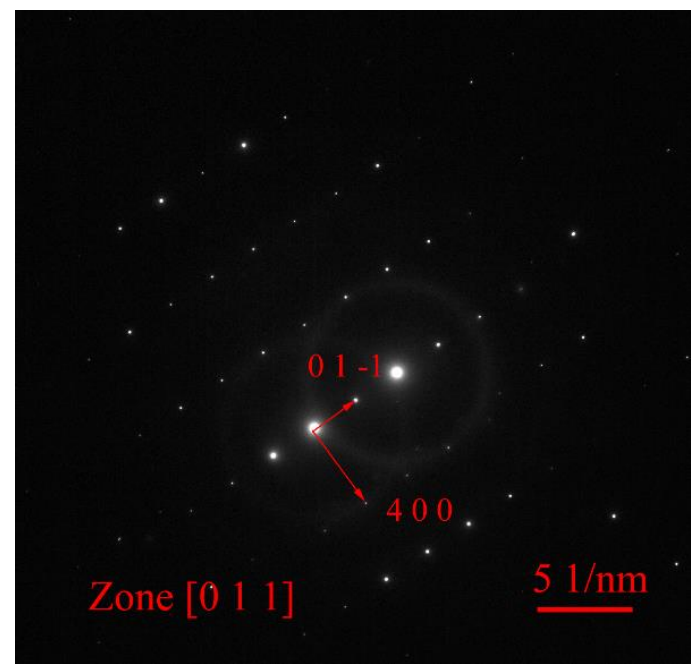

(b)

Figure 43. (a), (b) (a), (b) SAED patterns from the Cr-rich phase fitted using the tetragonal structure of $\sigma$ FeCr. The location of the SAED patterns is shown in Figure 40 

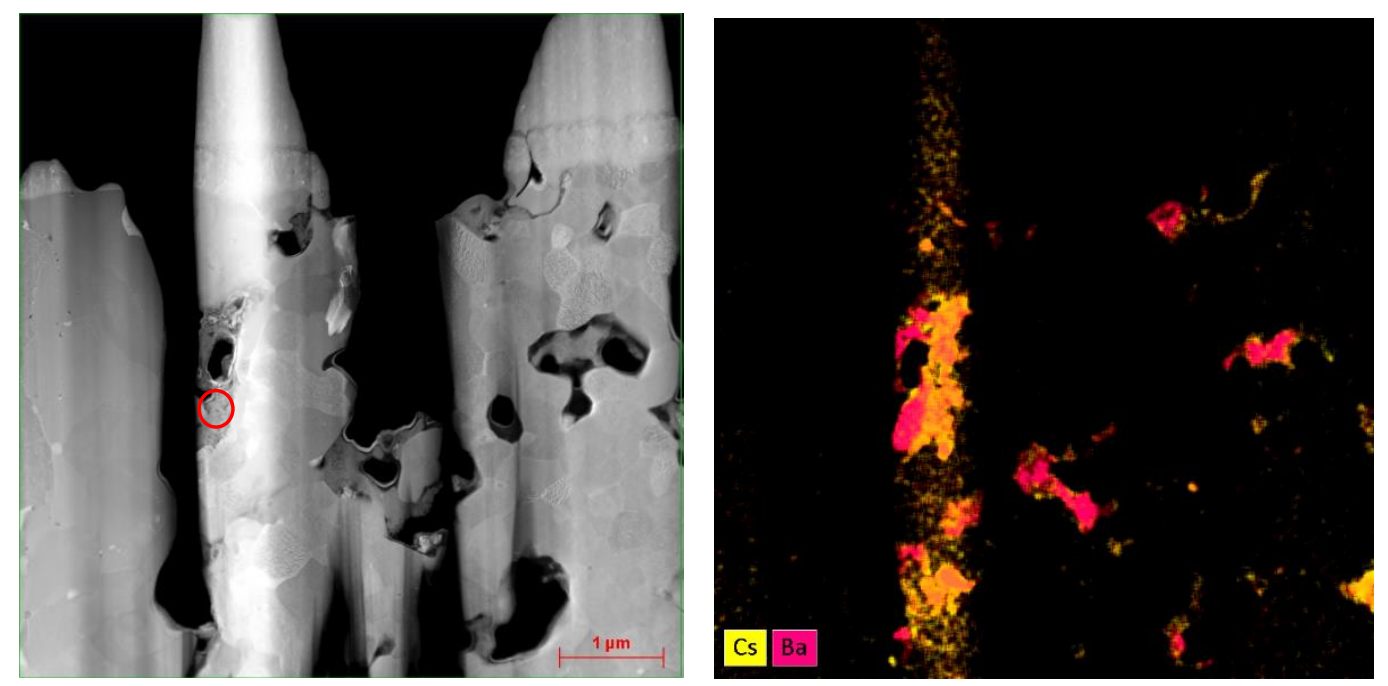

(a)
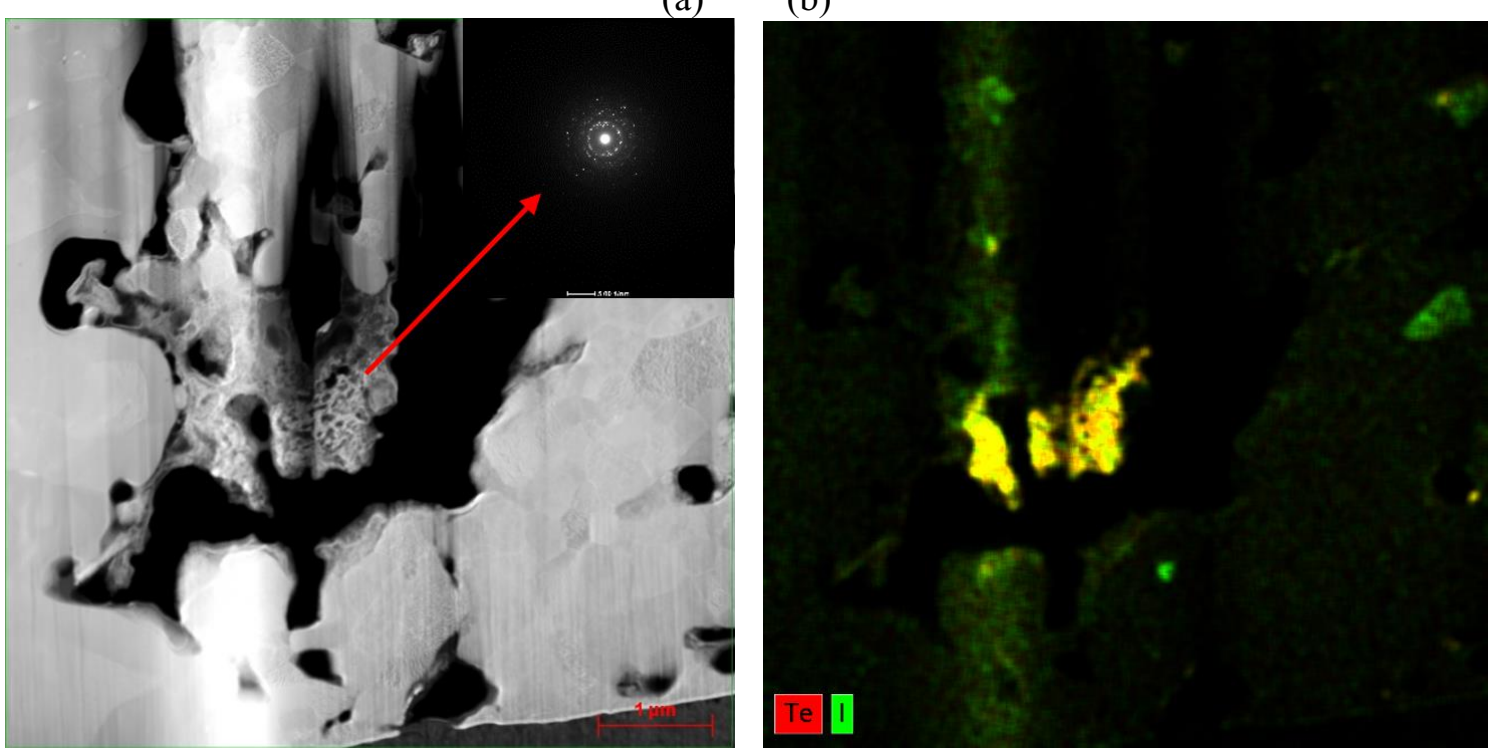

(c)

(d)

Figure 44. HAADF image of the (a) upper part of the lamella, (c) lower part of the lamella. (b), (d) EDS qualitative maps of the most prominent fission products in the two parts, respectively. The inset shows the nanocrystalline structure of the Te and I-rich region. 


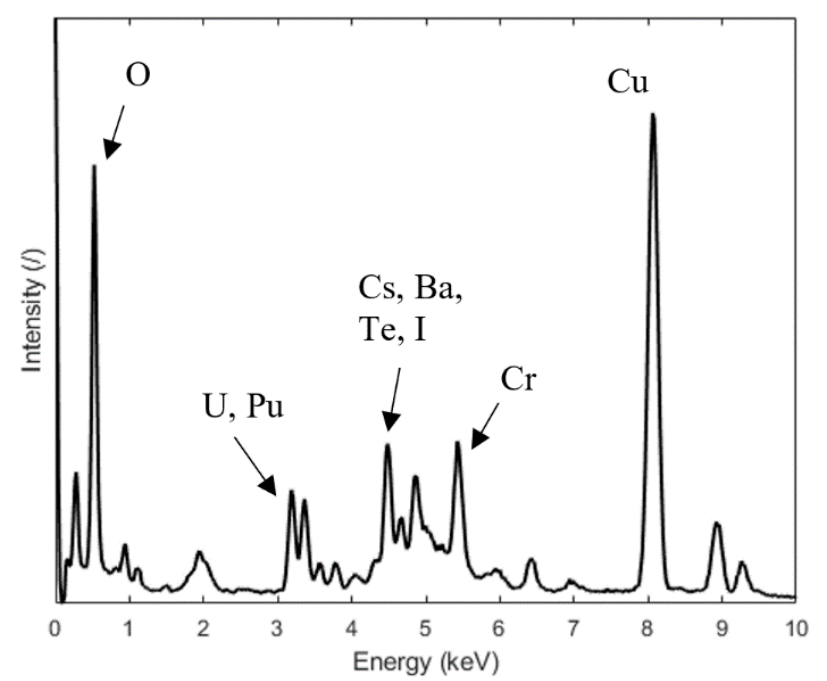

Figure 45. EDS spectrum of the nanocrystalline precipitate in the red circle in Figure 44a. The $\mathrm{Cu}$ signal comes from the TEM grid

Table 2. Composition of the precipitate circled in Figure 44a.

\begin{tabular}{|c|c|c|}
\hline Element & Atomic concentration $(\%)$ & Error $(\mathrm{at} \%)$ \\
\hline Oxygen & 35.47 & 3.52 \\
\hline Barium & 20.39 & 5.48 \\
\hline Chromium & 11.93 & 3.49 \\
\hline Yttrium & 10.07 & 3.63 \\
\hline Uranium & 9.95 & 10.16 \\
\hline Plutonium & 4.36 & 10.36 \\
\hline Cesium & 4.29 & 10.52 \\
\hline Iron & 2.70 & 5.04 \\
\hline Tellurium & 0.46 & 14.90 \\
\hline Palladium & 0.38 & 20.28 \\
\hline
\end{tabular}




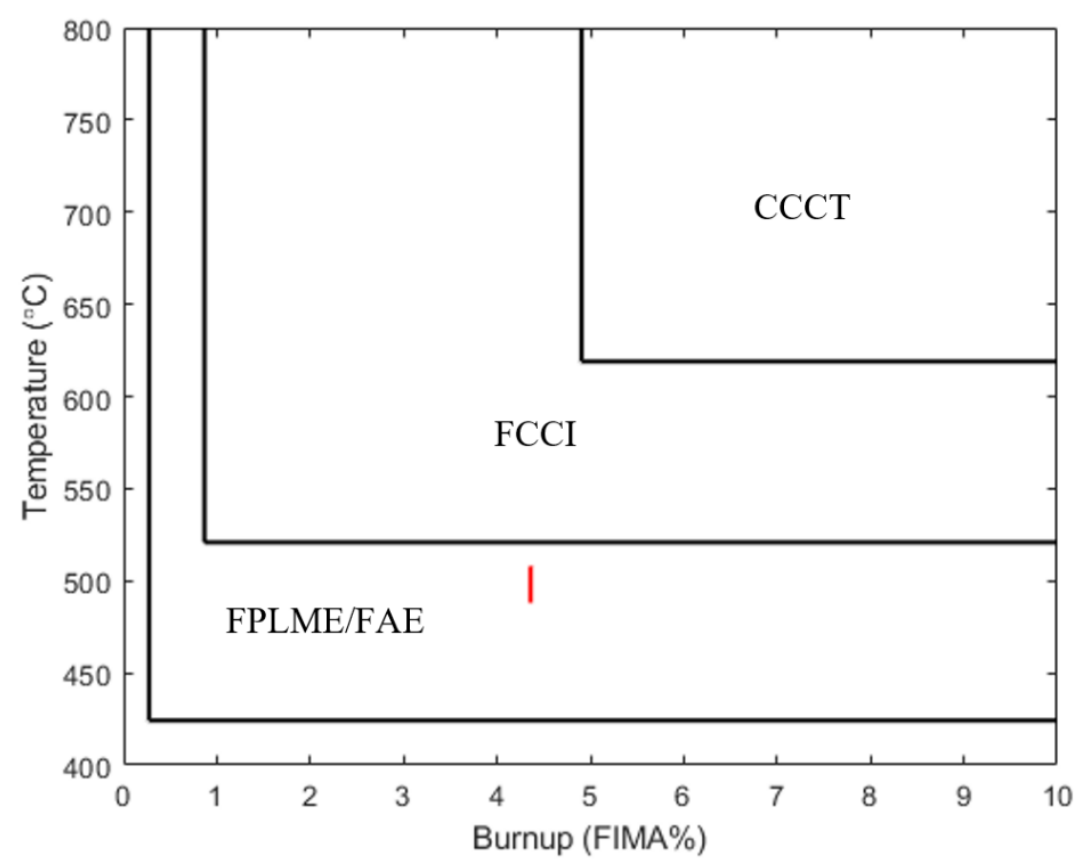

Figure 46. Comparison of the sample local conditions with the schematic of the primary environmental domains for the cladding interaction mechanisms. The red bar represents the range of expected temperature at the cladding inner surface for the current sample.

Another area of interest showing extensive interdiffusion between fuel and cladding is the one previously shown on the right side of Figure 36, reported at increased magnification below in Figure 47, together with EDS spectra at the specific locations indicated. Cr has extensively diffused into the fuel portion. Presence of $\mathrm{Cs}(+\mathrm{Ba})$ indicate that $\mathrm{Cs}_{2} \mathrm{CrO}_{4}$ could have formed, but cesium uranoplutonate (i.e., the family of compounds $\left.\mathrm{Cs}_{\mathrm{x}}(\mathrm{U}, \mathrm{Pu})_{\mathrm{y}} \mathrm{O}_{\mathrm{z}}\right)$ might also be present. A TEM lamella was extracted where the blue rectangle is drawn, and an overview is reported in Figure 48. The left side of the lamella is mainly composed of MOX, while on the right side a Cr-enriched area is present..

The area on the right of the lamella resembles the Cr-rich region at the interface with HT-9 seen in the previous lamella. Among the $\mathrm{Cr}$ and $\mathrm{Fe}$ phase, pockets of nanocrystalline material mainly composed by fission products are seen. High magnification images together with compositional information are reported in Figure 49, Table 3 to Table 5 and Figure 50. As seen previously, depending on local conditions, the composition of the fission product deposits changes locally. A zone highly enriched with Cs $(>65$ at $\%)$ was seen in Figure 50. SAED pattern collected in this area (Figure 50d) are compatible with the cubic structure of $\mathrm{Cs}$, but the final determination of the structure is uncertain from the rings. 


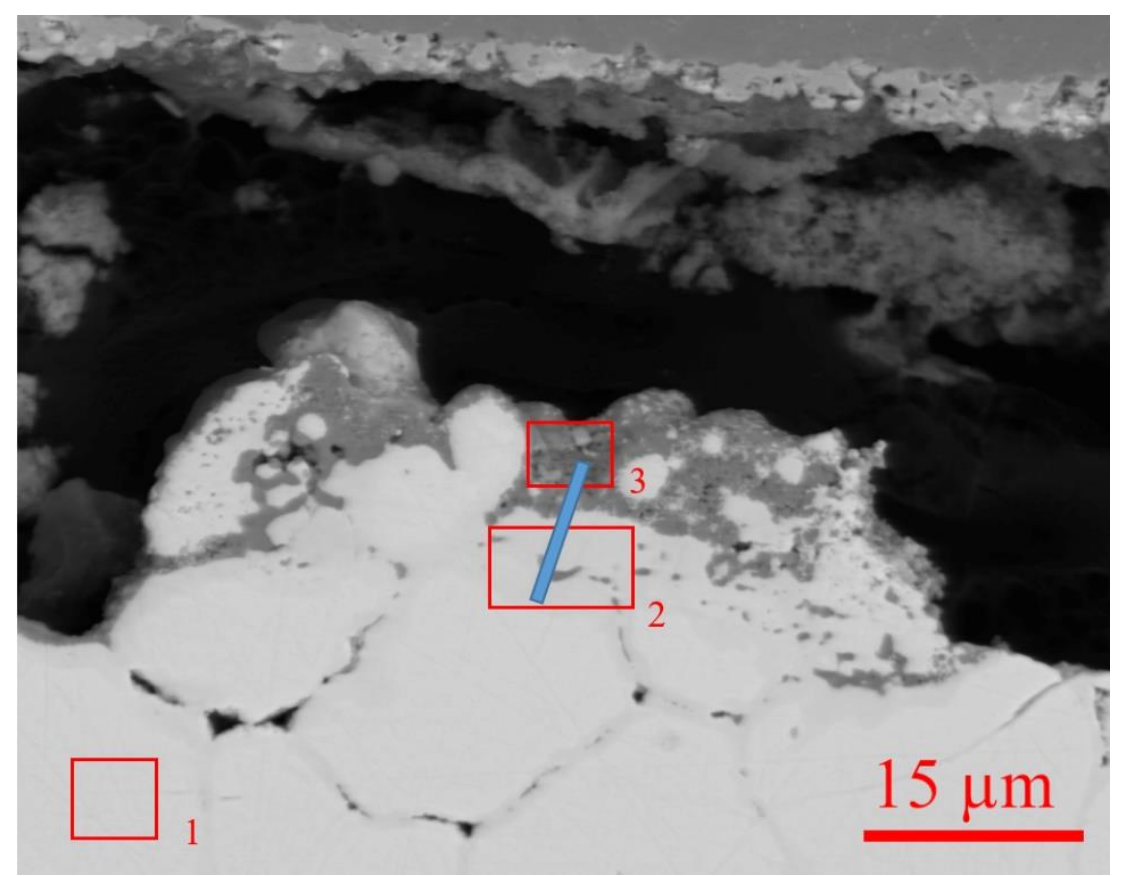

(a)

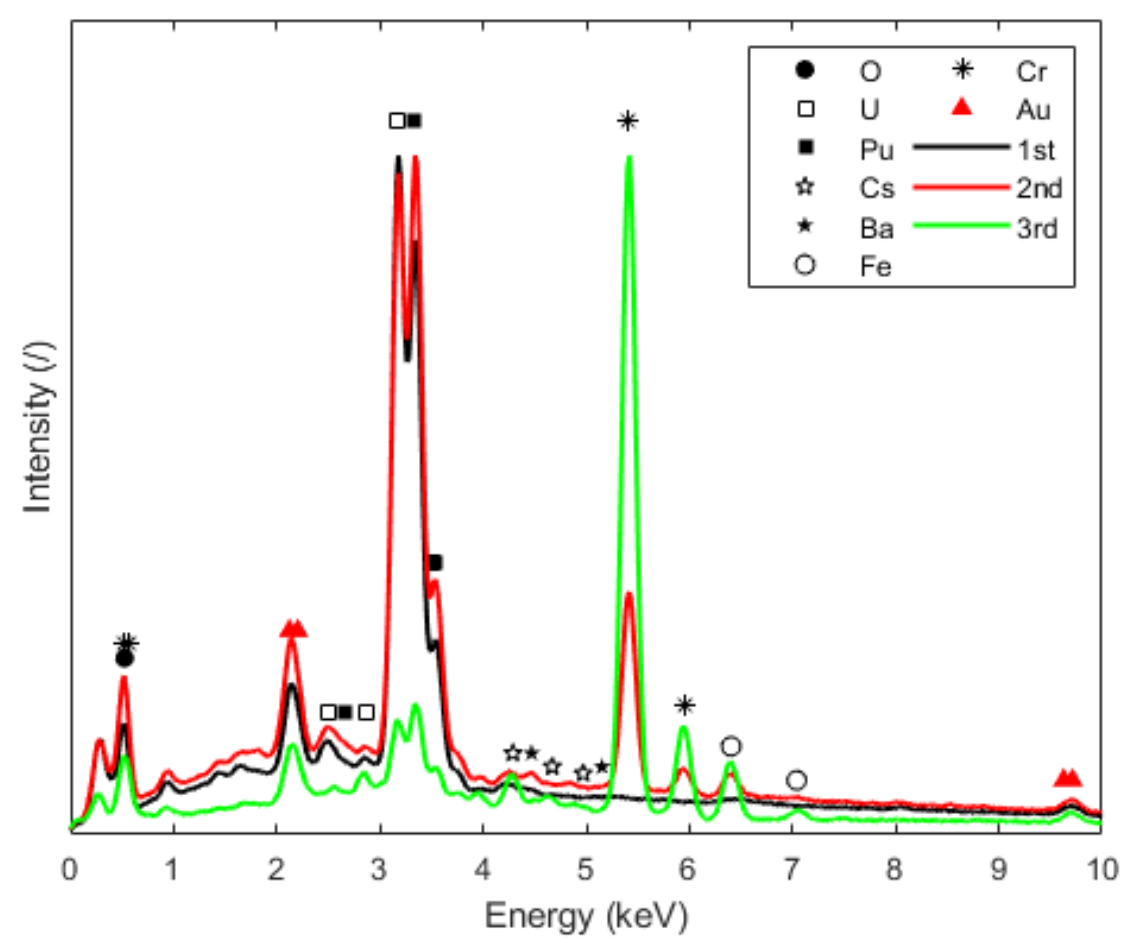

(b)

Figure 47. (a) High magnification of the fuel extreme periphery from Figure 36. (b) EDS spectra corresponding to the areas marked by the red rectangles in (a). The blue rectangle indicates the position of the TEM lamella. 


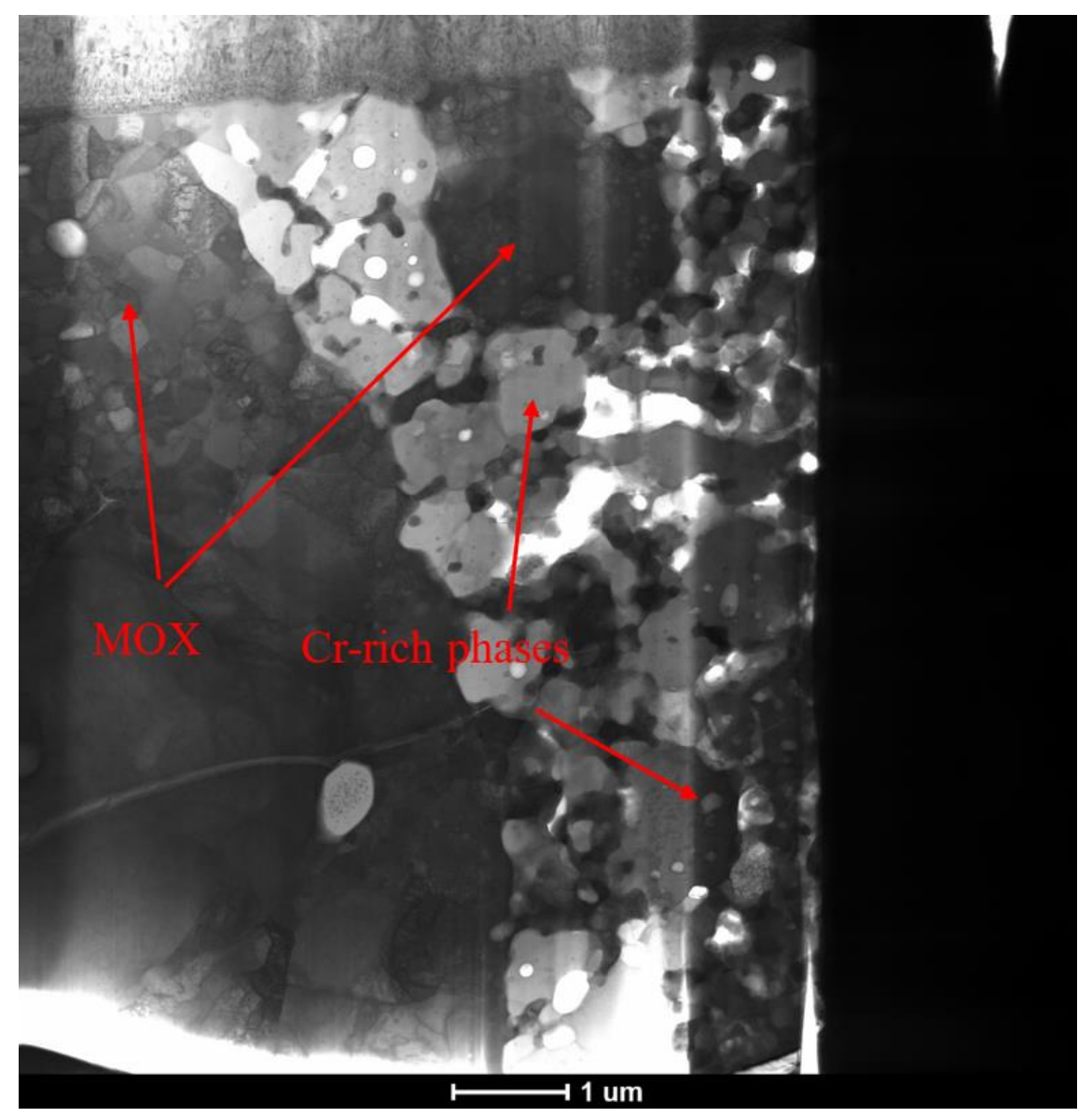

Figure 48. Overview of the lamella from Figure 47. 


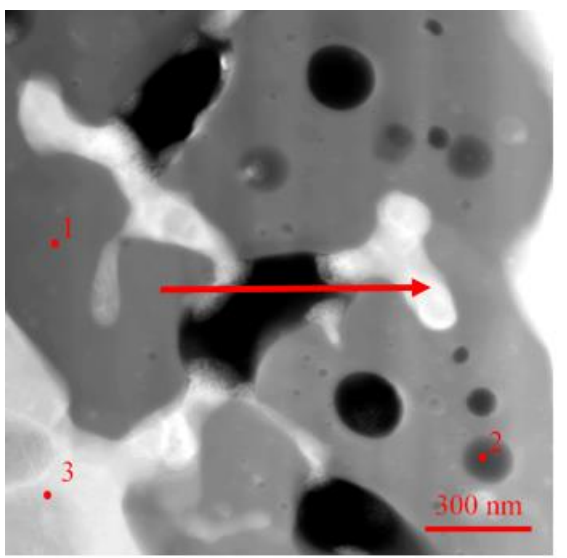

(a)

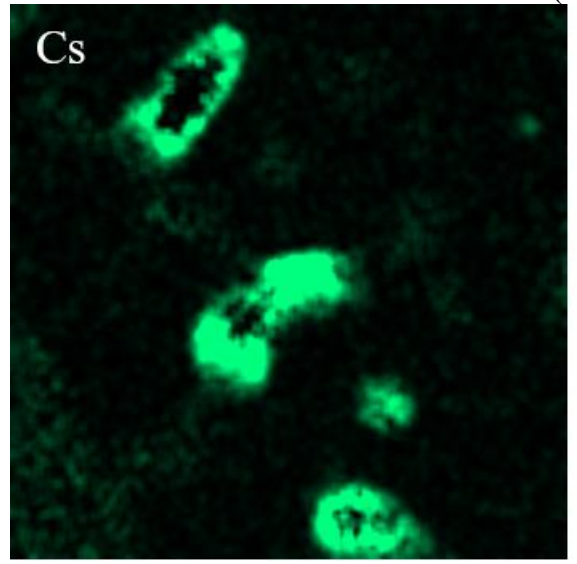

(c)

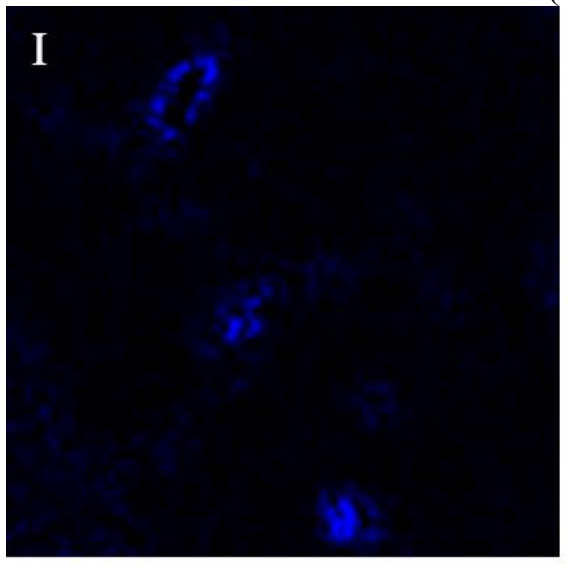

(e)

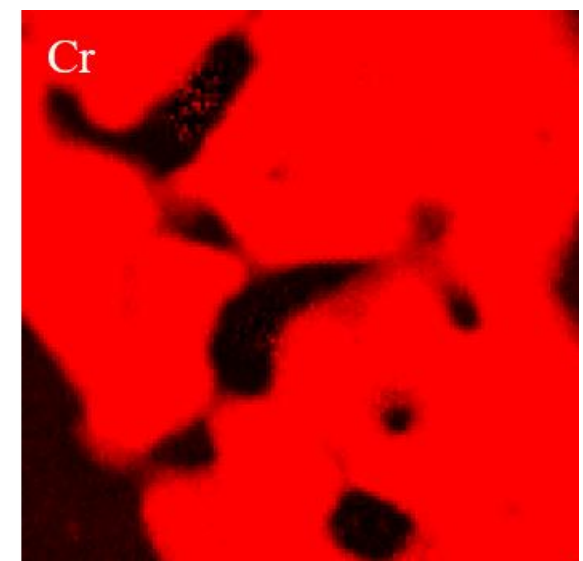

(b)

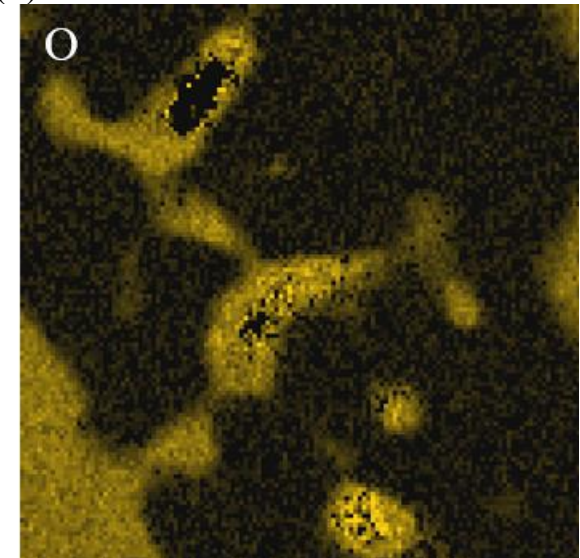

(d)

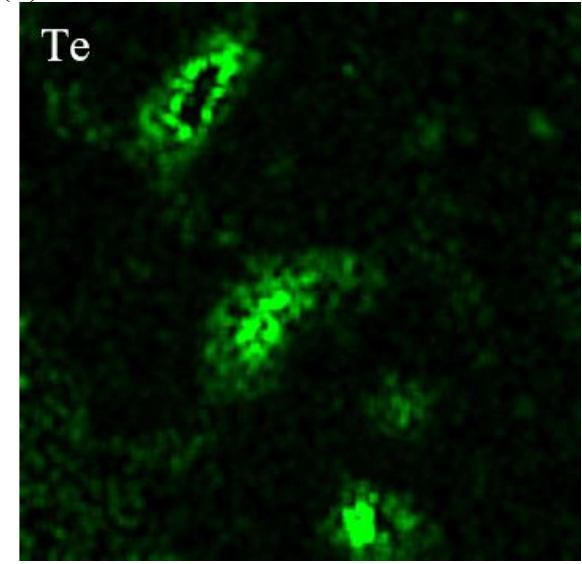

(f) 


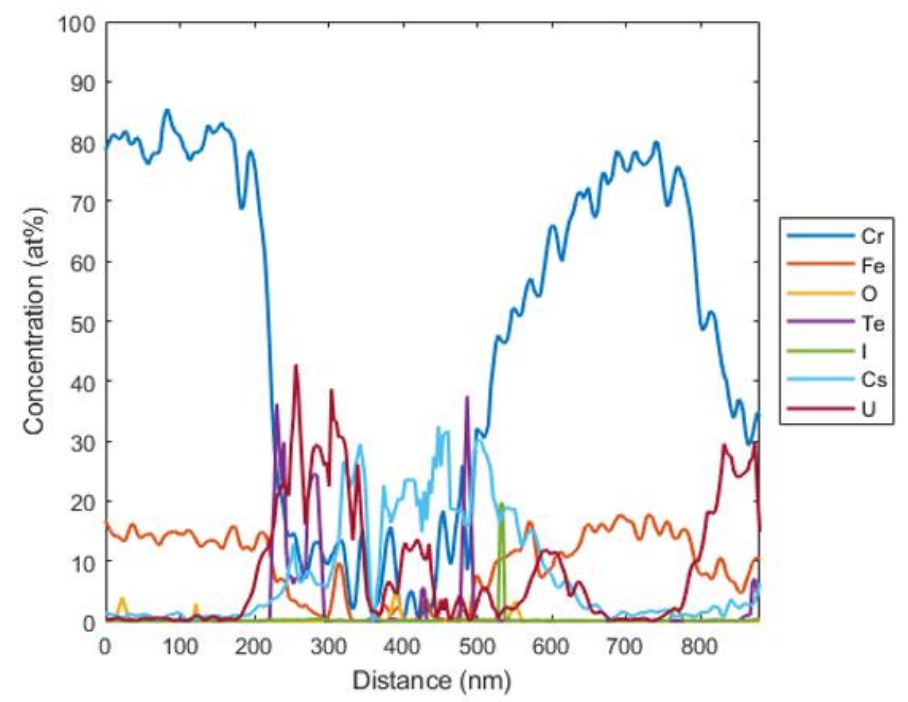

(g)

Figure 49. (a) HAADF image of the fission product precipitates in between the Cr-Fe areas. (b)-(f) Atomic maps of the major components. (g) Line scan from (a).

Table 3. Composition of location 1 in Figure 49a.

\begin{tabular}{lcc}
\hline & Concentration (at\%) & Error (at\%)* \\
\hline Chromium & 78.99 & 2.27 \\
Iron & 14.63 & 0.48 \\
Molybdenum & 2.85 & 0.21 \\
Ruthenium & 1.19 & 0.13 \\
Technetium & 0.58 & 0.09 \\
Zirconium & 0.43 & 0.07 \\
Oxygen & 0.41 & 0.03 \\
Rhodium & 0.29 & 0.07 \\
Cobalt & 0.24 & 0.04 \\
Nickel & 0.20 & 0.04 \\
Palladium & 0.11 & 0.06 \\
\hline * The error represents a 60\% confidence interval & & \\
Table 4. Composition of location 2 in Figure 49a. & \\
\hline & Concentration (at\%) \\
\hline Chromium & 49.76 & \\
Uranium & 12.42 & Error (at\%) \\
Cesium & 11.45 & 0.83 \\
Oxygen & 10.88 & 3.04 \\
Plutonium & 5.89 & 1.58 \\
Iodine & 3.93 & 0.09 \\
Tellurium & 2.85 & 1.51 \\
Barium & 2.82 & 0.68 \\
\hline
\end{tabular}


Table 5. Composition of location 3 in Figure 49a.

\begin{tabular}{lcc}
\hline & Concentration $(\mathrm{at} \%)$ & Error $(\mathrm{at} \%)$ \\
\hline Uranium & 40.88 & 5.16 \\
Plutonium & 31.31 & 4.06 \\
Oxygen & 9.45 & 0.05 \\
Chromium & 8.79 & 0.10 \\
Iron & 3.95 & 0.06 \\
Barium & 2.63 & 0.22 \\
Cesium & 1.80 & 0.15 \\
Tellurium & 1.19 & 0.11 \\
\hline
\end{tabular}
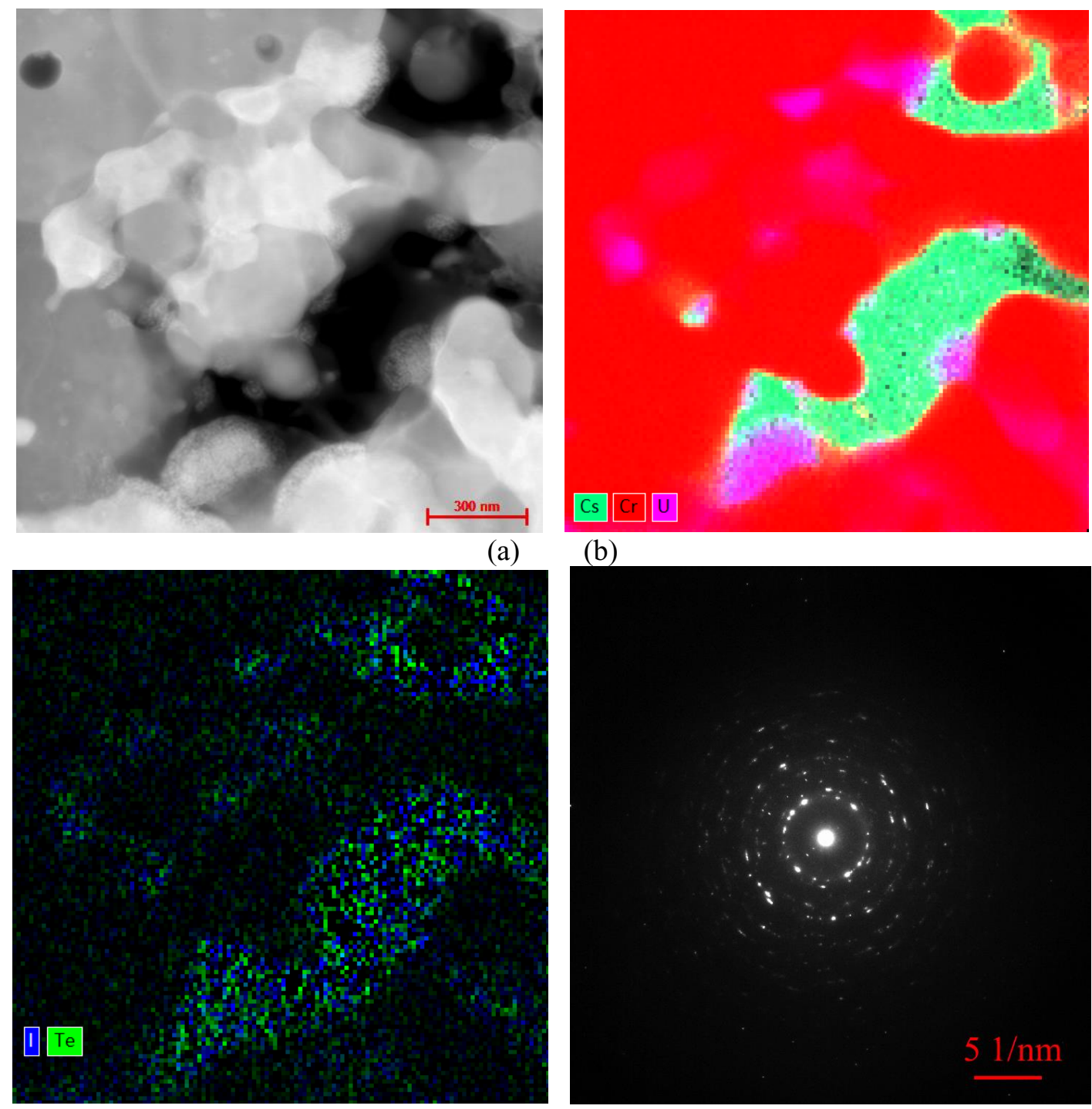

(c)

(d)

Figure 50. (a) HAADF from a second location containing the fission products. (b)-(c) Atomic maps of major components. (d) SAED pattern corresponding to the Cs-rich area. 


\subsection{Pin L08}

\subsubsection{Sample 3}

\subsubsection{Fuel microstructure and fission products}

The overview of the sample is shown in Figure 51. First, chemical composition of fission product precipitates was measured across the radius, focusing on the five metal precipitates (FMPs). Representative micrographs are shown in Figure 52a, b and c for the center, mid radius (columnar grain region) and close to the edge, respectively. As can be seen from Table 6, close to the central void, the composition was constant among the precipitates, while in the columnar region the content varied significantly, as proved by the large standard deviation. At the edge, FMP could still be found (see arrow in the figure), but the larger precipitates like the one shown in Figure 52c contained Mo, Cs and $U$ in oxide form. The different composition is documented in the spectra comparison in Figure 53.

Complementary EPMA measurements have been performed along the radius shown in the solid red box in Figure 51. As already noted from the EDS measurements, the composition is scattered along the radius. Mo concentration is decreasing in the equiaxed grain region, while enriched precipitates have been measured at the fuel periphery, sign of radial migration of the specie.

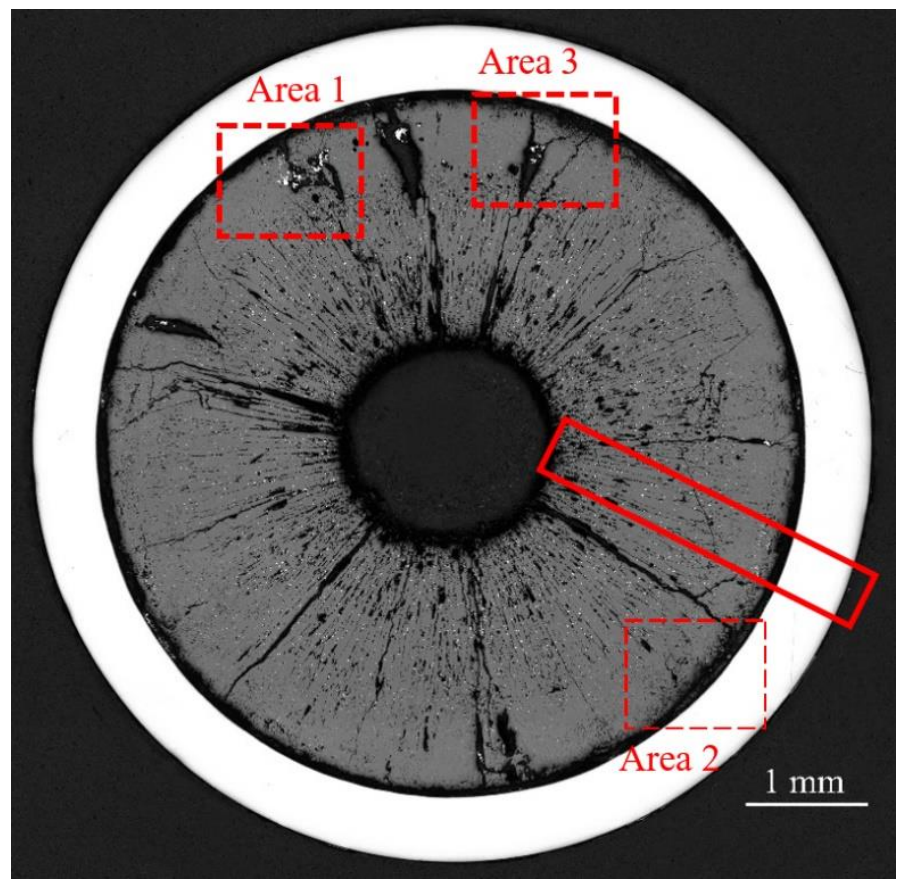

Figure 51. Optical microscopy overview of sample 3. The dashed boxes 1 and 3 indicate the areas where the SEM/TEM analyses were focused, while area 2 indicates an additional area analyzed by EPMA. The solid rectangle indicates the region where EPMA radial quantitative analyses were performed. 


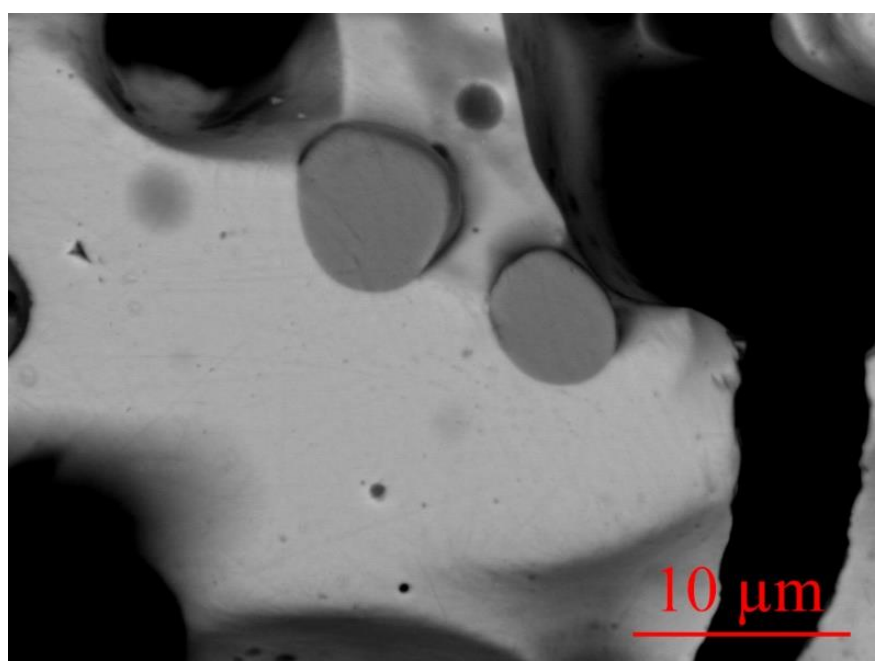

(a)

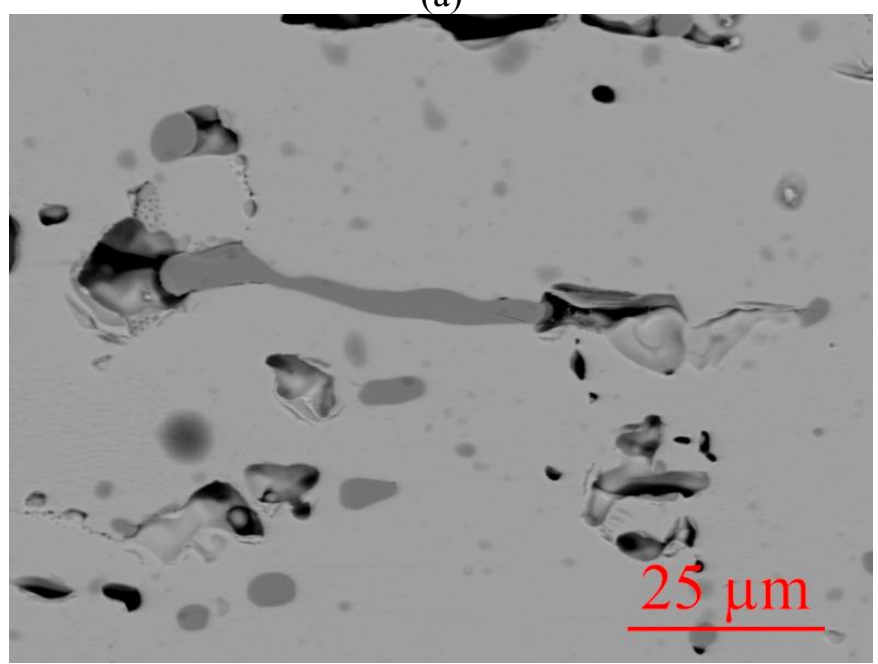

(b)

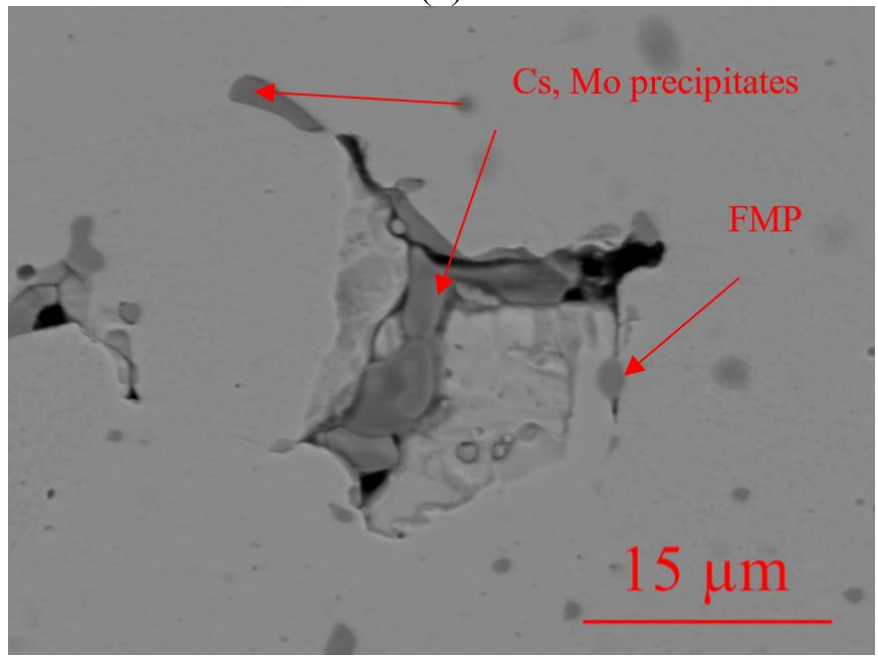

(c)

Figure 52. Micrographs showing fission products inclusions at different radial locations. (a) Close to the central void. (b) In the middle of the columnar grain area. (c) Close to the pellet edge. 
Table 6. Chemical composition of the fission product precipitates at different radial locations.

\begin{tabular}{lccc} 
Element & $\begin{array}{c}\text { Center } \\
\text { wt } \%\end{array}$ & $\begin{array}{c}\text { Mid radius } \\
\text { wt } \%\end{array}$ & $\begin{array}{c}\text { Edge } \\
\text { wt \% }\end{array}$ \\
\hline $\mathrm{Mo}$ & $31.57 \pm 0.43$ & $28.32 \pm 1.88$ & $42.13 \pm 0.42$ \\
$\mathrm{Tc}$ & $14.26 \pm 0.88$ & $2.55 \pm 1.74$ & $5.37 \pm 0.38$ \\
$\mathrm{Ru}$ & $44.96 \pm 0.56$ & $16.91 \pm 8.89$ & $29.64 \pm 0.44$ \\
$\mathrm{Rh}$ & $0.61 \pm 0.60$ & $2.59 \pm 1.63$ & $7.05 \pm 0.46$ \\
$\mathrm{Pd}$ & $8.66 \pm 0.36$ & $49.62 \pm 13.88$ & $15.8 \pm 0.37$ \\
\hline
\end{tabular}

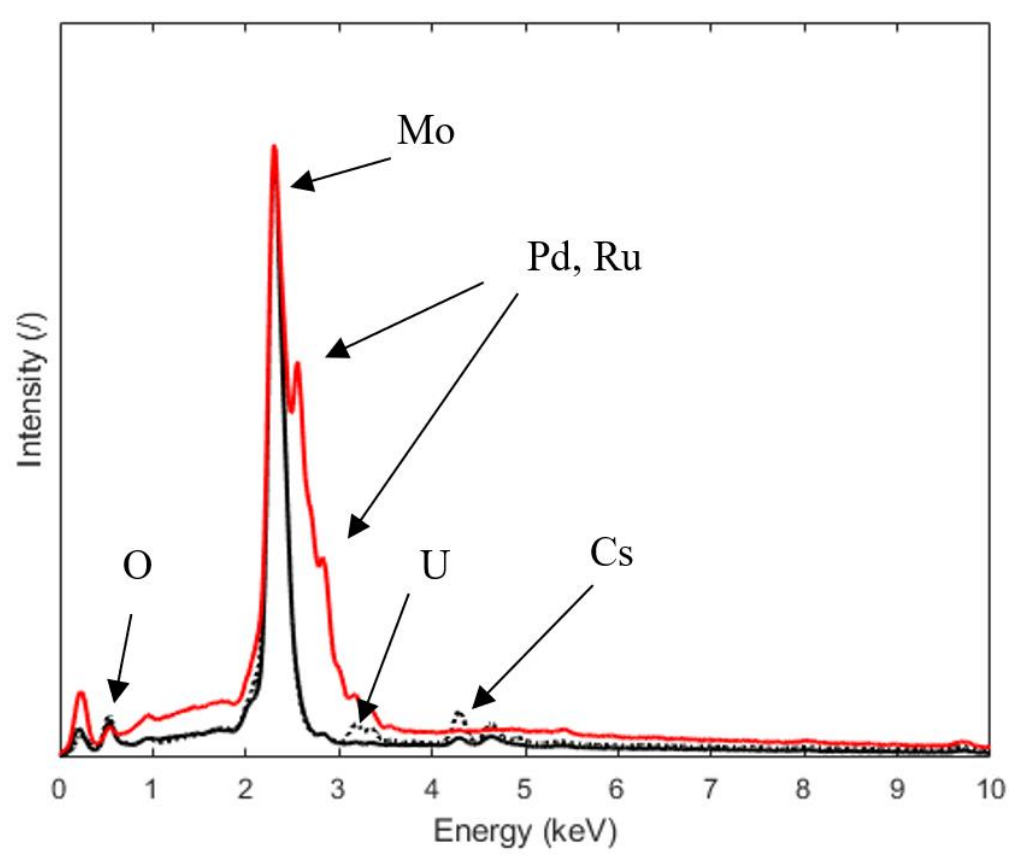

Figure 53. Spectra comparison between the FMP and the Mo and Cs- bearing precipitate at the pellet periphery from Figure 52c. 


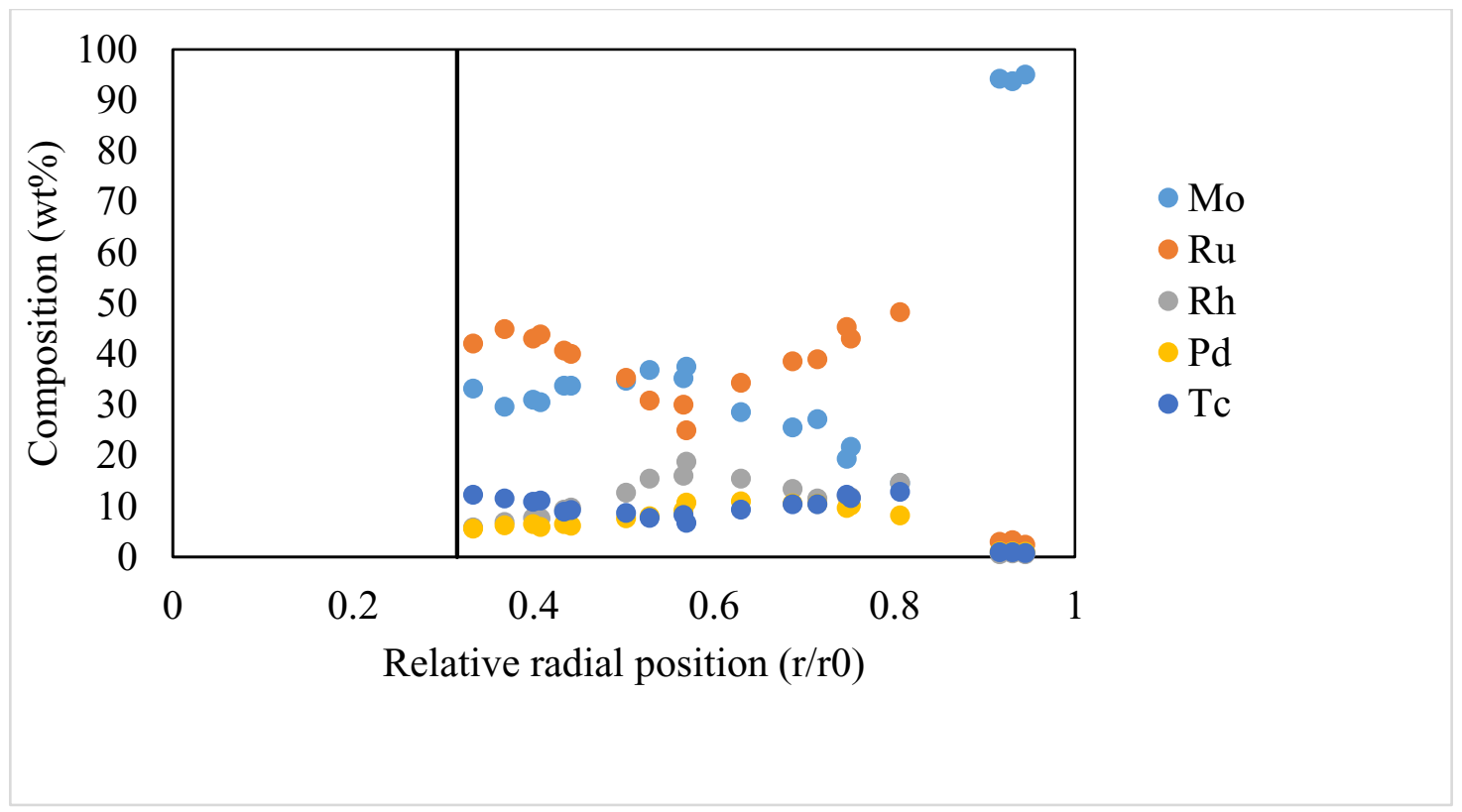

Figure 54. Additional compositional measurements of the FMP measured with EPMA.

Regions showing large agglomerations of fission products were seen already from the optical examinations (see boxes in Figure 51) and were further analyzed via SEM, EPMA and TEM. A BSE image of the area in box 1 is shown in Figure 55. Initial scoping analyses were performed via SEM/EDS. The composition showed predominantly Mo and Pd, but variations in the composition and segregation of the species was observed within the precipitates, which can be qualitatively appreciated from the maps in Figure 56. A larger area map is reported in the next figure, Figure 57. The initial observations regarding the composition variability of the metallic precipitates are further highlighted by the EPMA maps showing weight percent distributions. Minor elements like Te and $\mathrm{Zr}$ concentrated in specific precipitates or close to them were also detected. Association of Te with Pd or other metal phases has been already shown in previous work [2], and it is not surprising. Co-localization of metallic phases and grey phases (represented by $\mathrm{Zr}$ and $\mathrm{Ba}$ in Figure 57 ( $\mathrm{f}$ and $\mathrm{g}$ ) has also been observed in TEM, which is presented in the next section. The presence of Cs remains questionable, as it is distributed mainly along the cracked fuel grains, which could create spectrum distortion due to edge effects. However, the volatile nature of Cs could favor its distribution along boundaries, hence, the observed map could be partially accurate. Other species were detected by EPMA, specifically Fe, Ni and $\mathrm{Cr}$. The intensity of the maps for each of these elements was precisely coincident with the broken areas exposing the epoxy. The signal from cracked bottom surfaces is considered questionable. The sample is mounted in a stainless steel 316 holder; hence, it cannot be excluded that residual debris from grinding were trapped into the fuel cracked region. It is, therefore, the opinion of the authors, that the extensive presence of these species is an artifact from sample preparation.

A TEM lamella was extracted at the interface between one of the precipitates and the matrix (see blue box in Figure 56). The overview of the lamella (Figure 58) shows two main phases, the FMP on the top and the MOX matrix on the bottom. Within the MOX phase, a grey-phase precipitate is visible. From the EDS spectrum of the FMP in Figure 59a, we obtained the approximate composition of the precipitate and confronted with the theoretical phase diagram in Figure 59b. According to the prediction, the precipitate should have a hexagonal structure ( $\varepsilon$ phase). SAED patterns agreed well with the reported $\varepsilon$ structure in literature [7,30,31]. The calculated lattice parameters were $\mathrm{a}=2.78 \AA \pm 0.28 \AA$ and $\mathrm{c}=4.75 \AA \pm 0.34 \AA$. Twins were observed in the precipitate, as shown in Figure 60. The spectrum acquired from the grey phase (Figure 61) shows $\mathrm{Ba}, \mathrm{Zr}$ and $\mathrm{Cs}$ as main components, with presence of $\mathrm{Mo}, \mathrm{U}$ and $\mathrm{Pu}$. It was not 
possible to obtain a clear diffraction pattern from the precipitate, as it was smaller than the smallest aperture and large interference from the diffracting planes of the MOX matrix occurred. Similar composition was obtained from another grey-phase precipitate embedded at the interface between the MOX and the FMP (see Figure 62). Finally, spectra were acquired from two grains in the MOX matrix (Figure 63). A slight depletion of Pu was found in the location closer to the FMP (see Table 7), while the other location matches the expected Pu content after approximately 6\% FIMA. Both dislocation loops (Figure 64a) and dislocation lines pinned by grey-phase precipitates (Figure 64b) were observed, with increased concentration along the interface with the FMP.

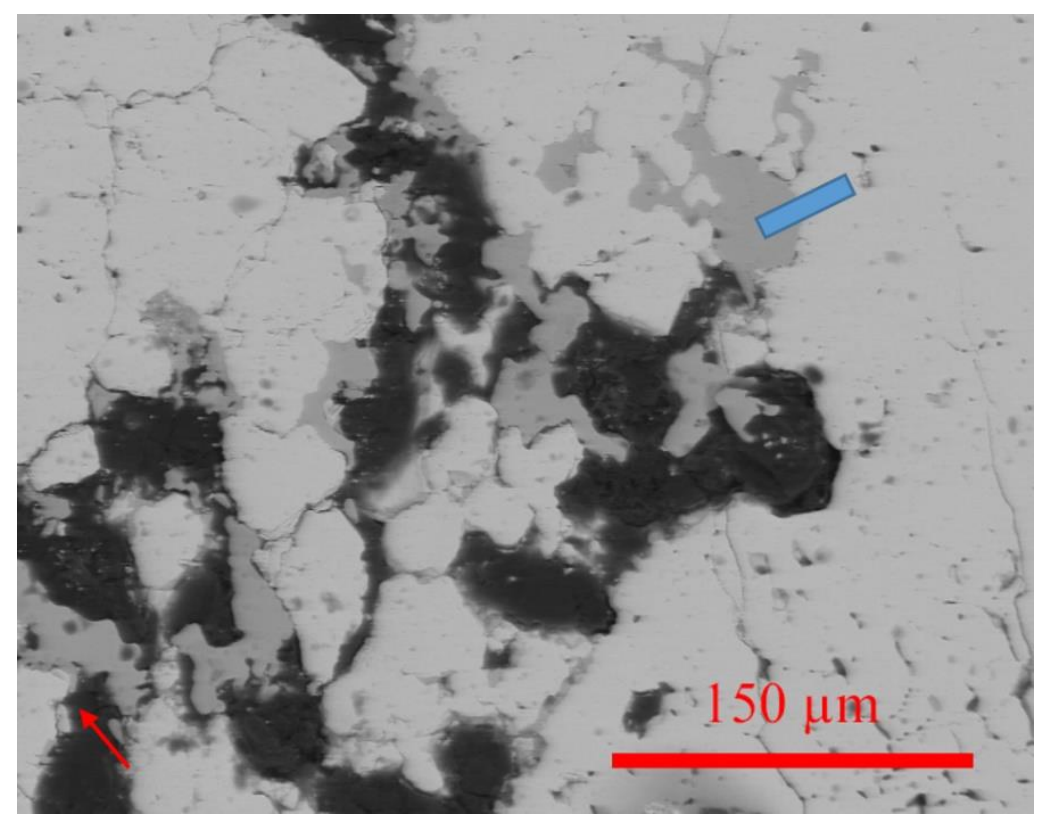

Figure 55, BSE image of the large metallic inclusions from area 1 in Figure 51. The rectangle indicates the location of the TEM lamella extracted in this region. The arrow indicates the region where species composition was observed. 


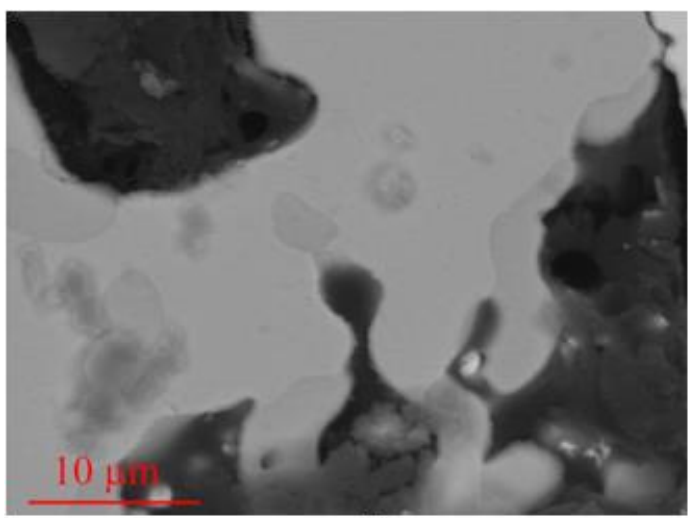

(a)

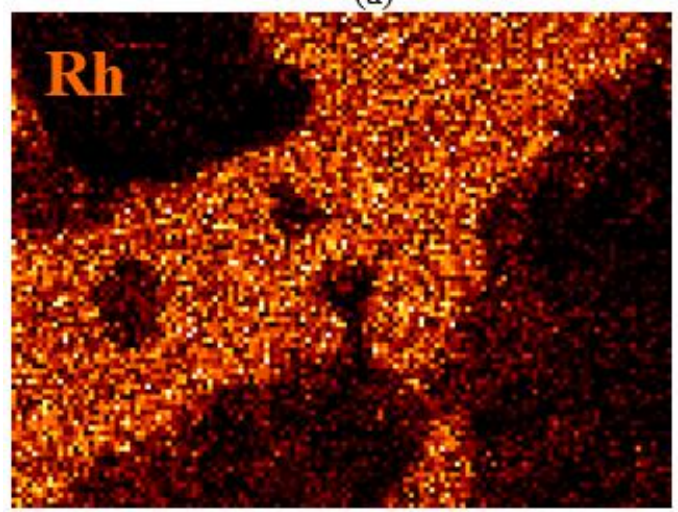

(c)

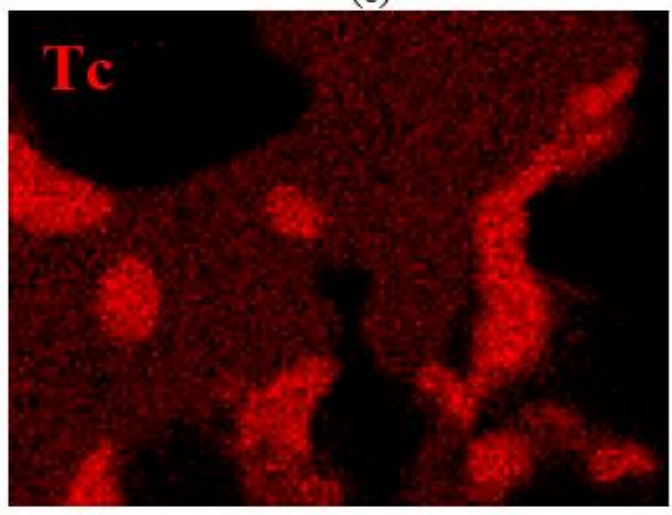

(e)

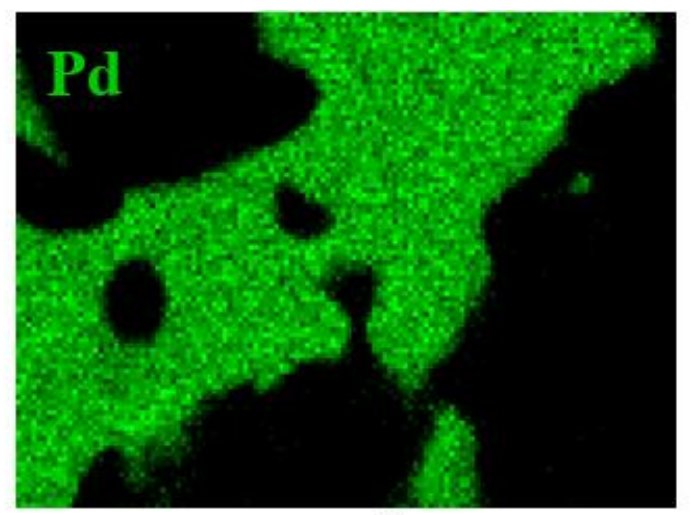

(b)

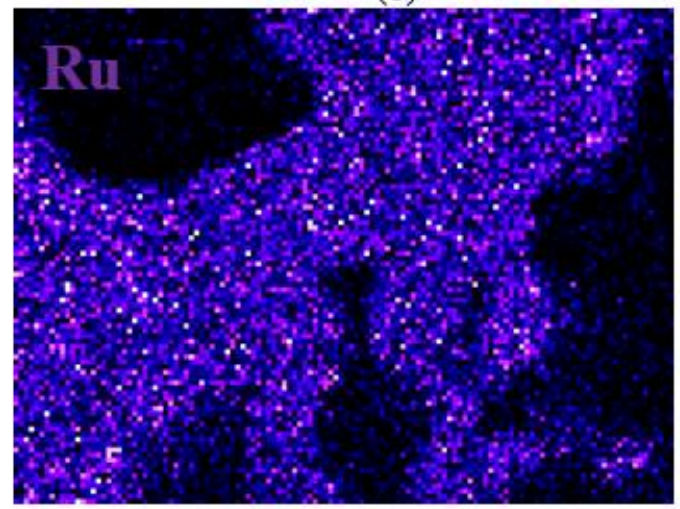

(d)

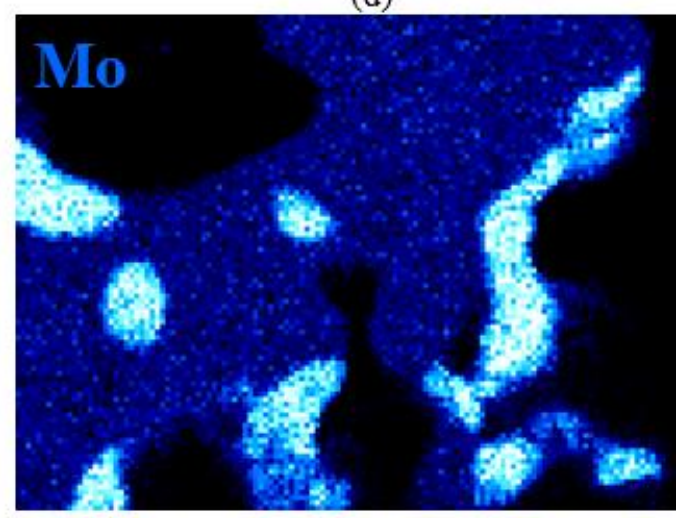

(f)

Figure 56. High magnification image of the region indicated by the arrow in Figure 55, showing metallic fission product segregation within the FMP. (a) BSE image, (b)-(e) EDS maps of the components of the FMP. 


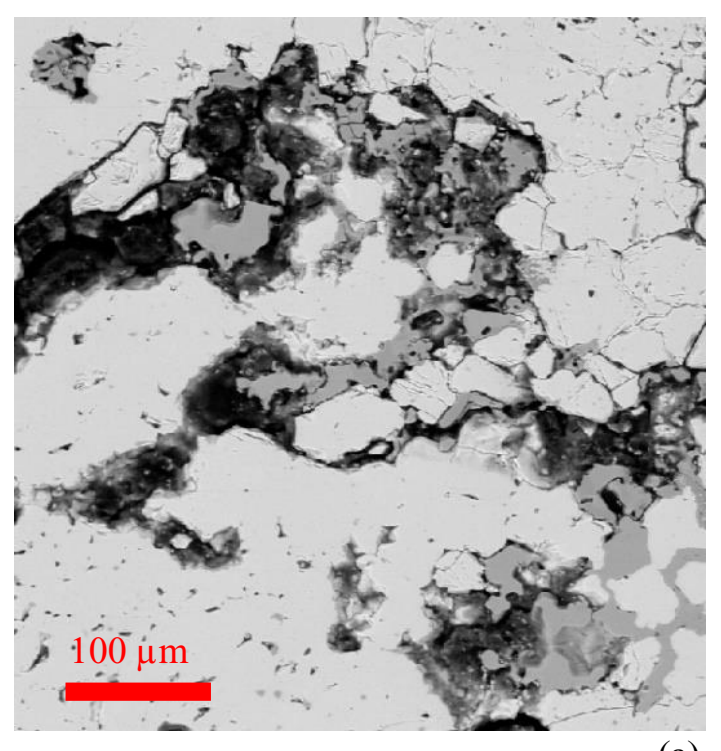

Pd Wt $\%$

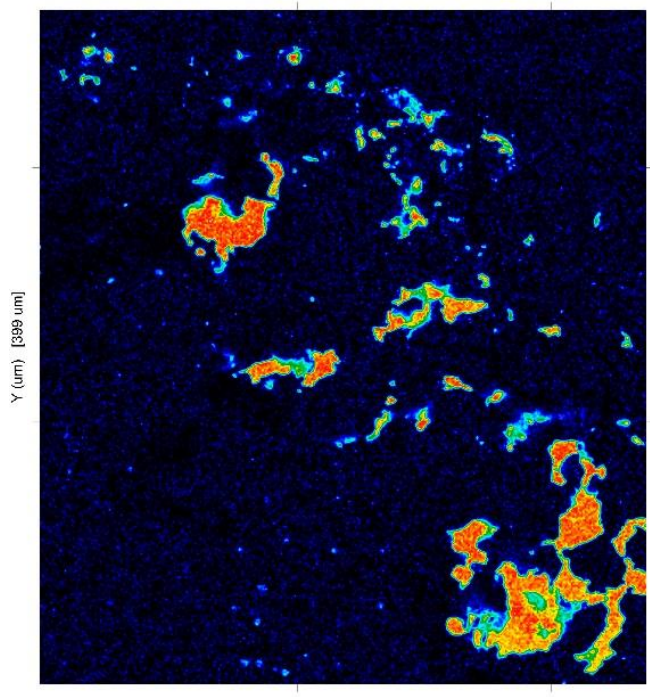

X(um) [359 um] (a)

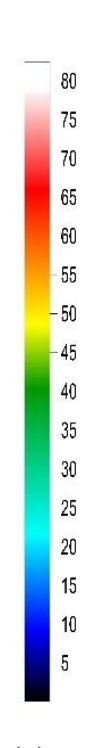

(c)
$\mathrm{Mo} \mathrm{Wt}_{\mathrm{t}} \%$

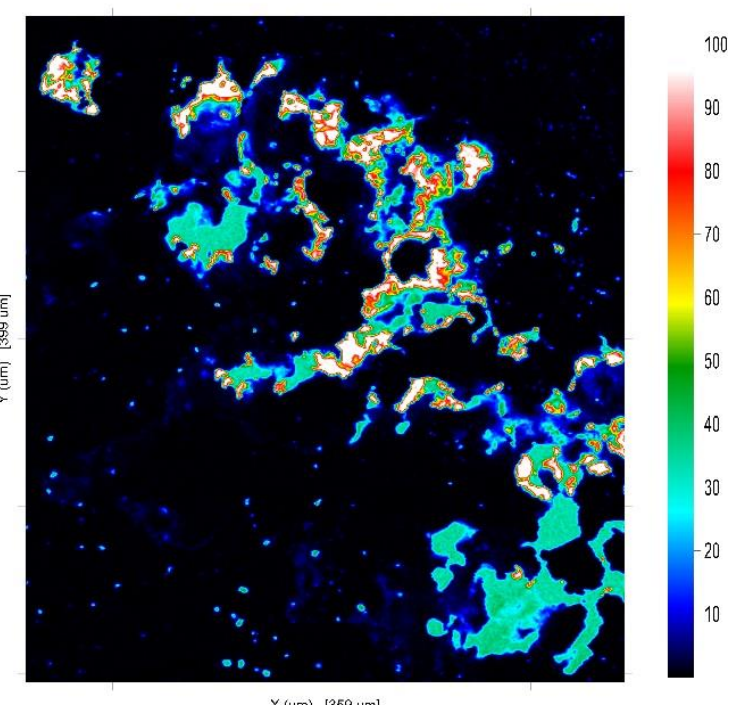

(b)

$\mathrm{Ru} \mathrm{Wt} \%$

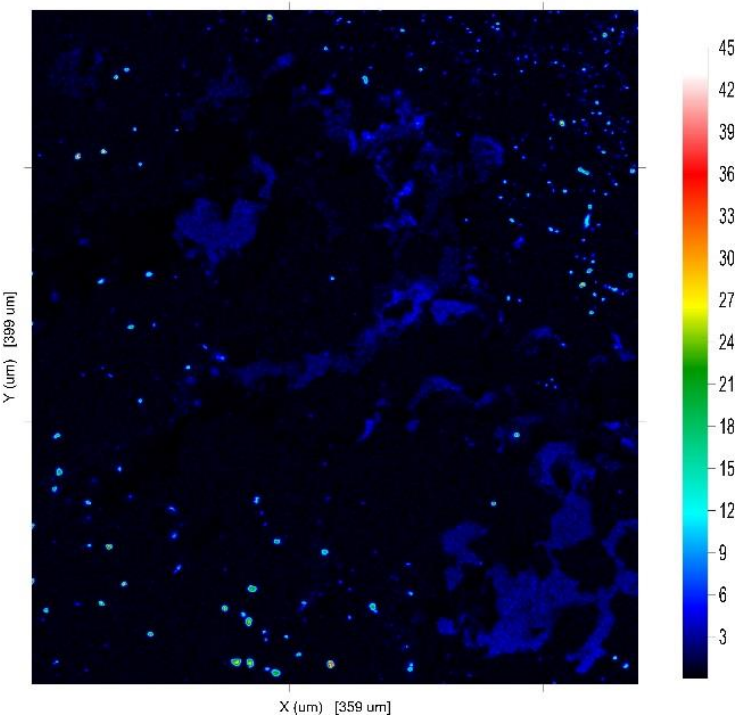

(d) 
$\mathrm{Te} \mathrm{Wt} \%$

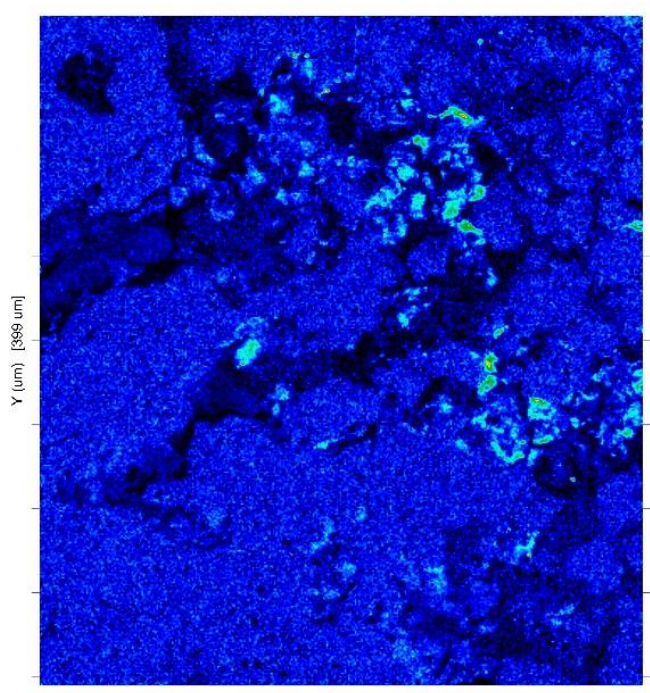

$X$ (urn) $[359$ um]

$\mathrm{Ba} \mathrm{Wt} \%$

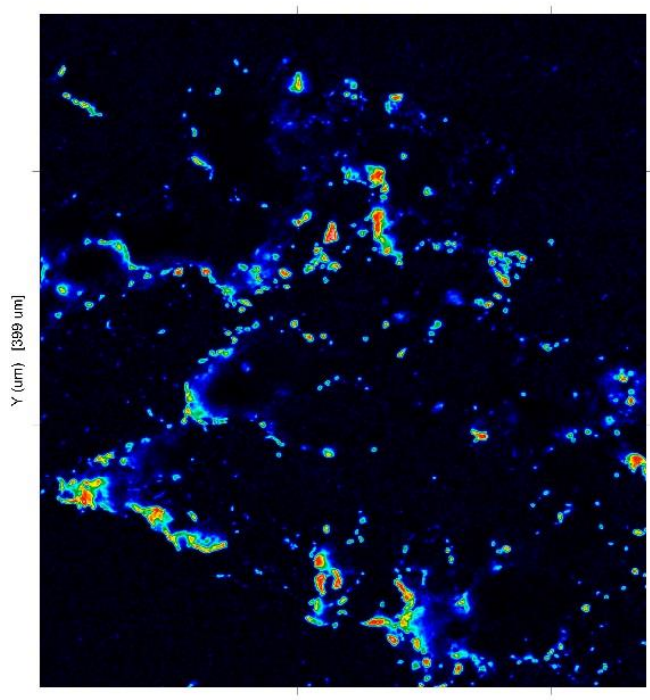

$\mathrm{X}$ (um) $[359 \mathrm{um}]$

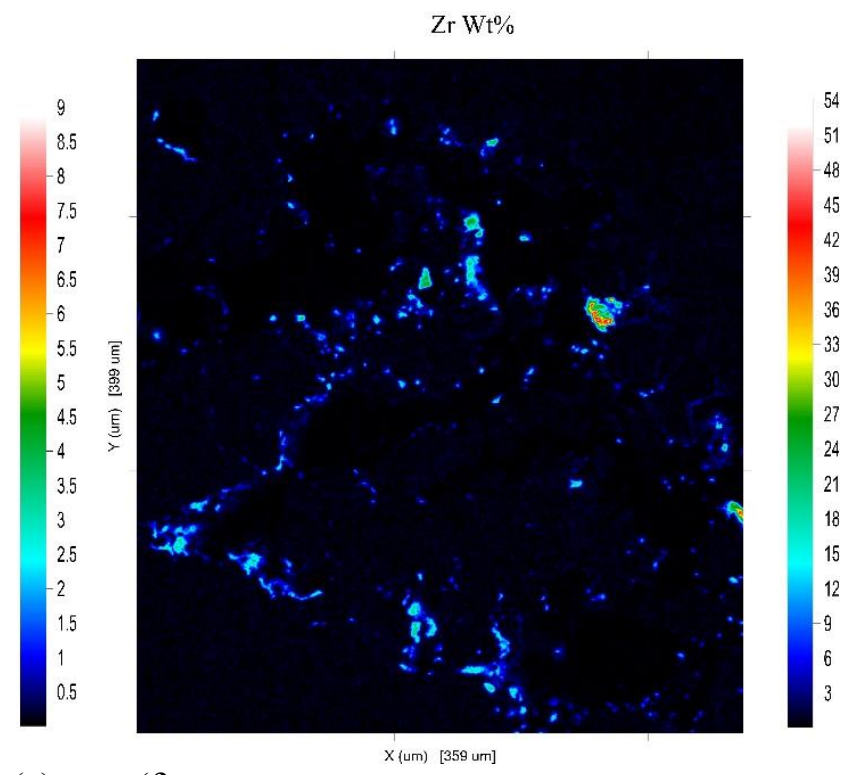

(e)

(f)

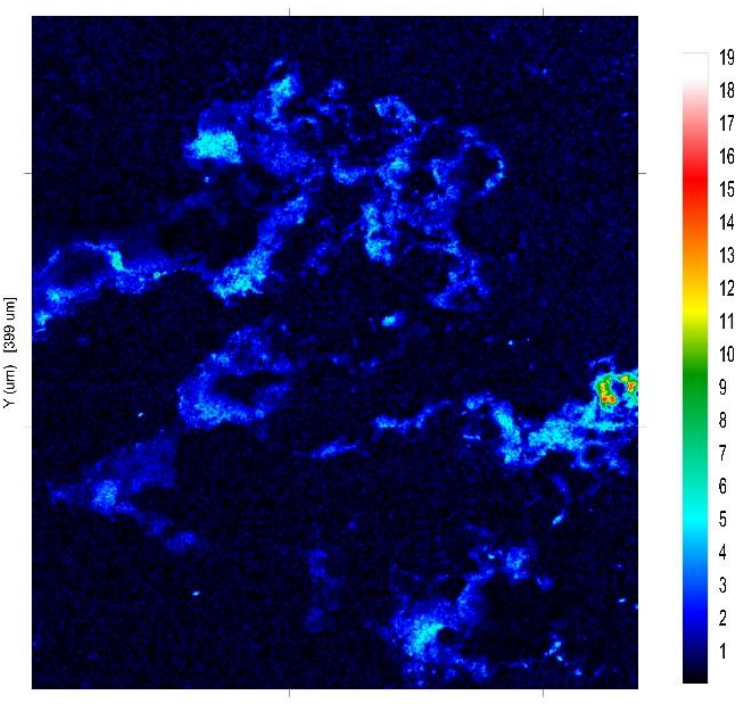

(h)

Figure 57. (a) BSE overview and (b)-(h) chemical maps from EPMA over the area showing extensive fission product precipitation at the fuel periphery. 


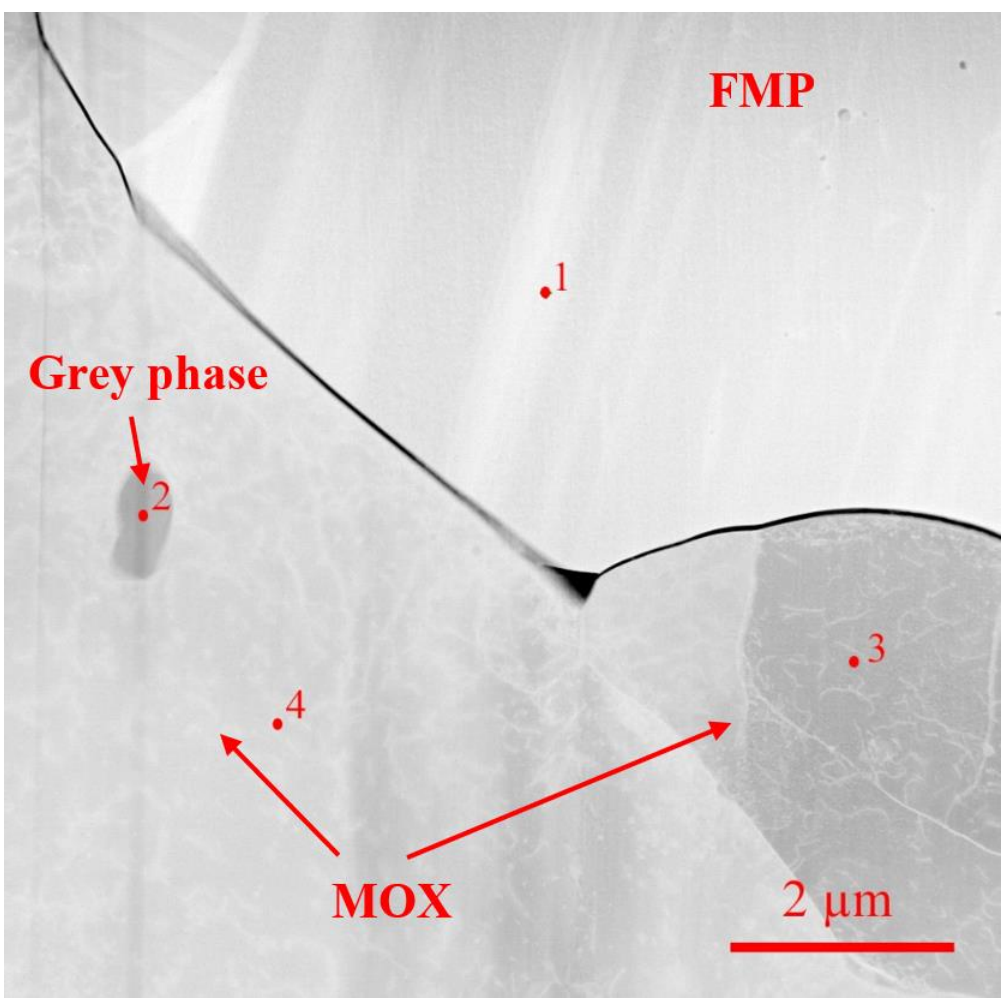

Figure 58. HAADF image of the lamella, showing the various phases. The points indicate the location of the EDS spectra shown in the next figures. 


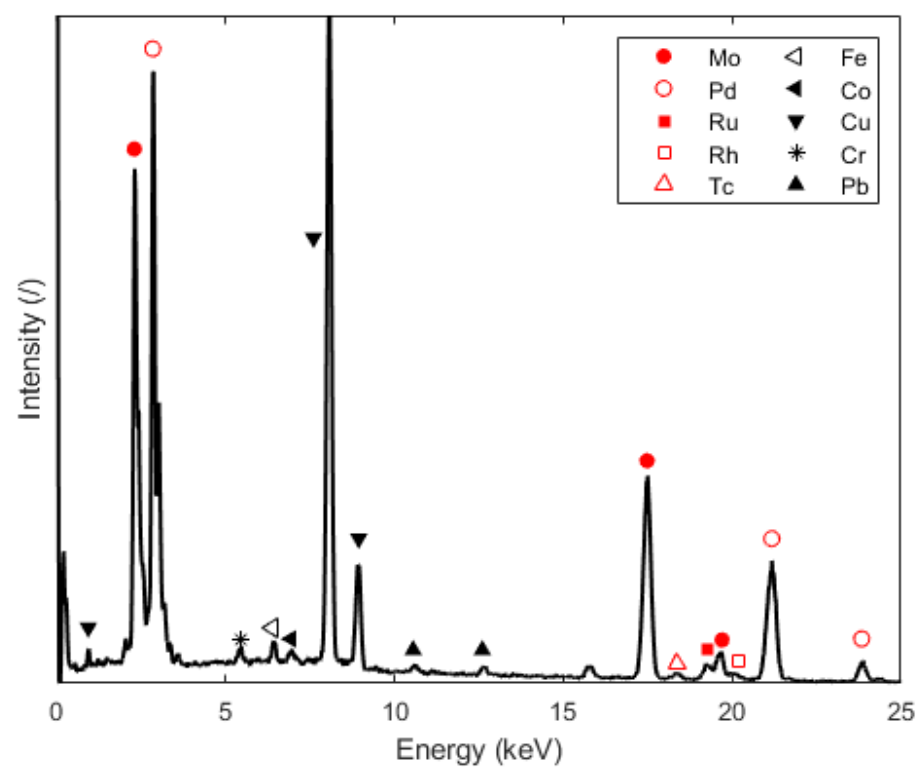

(a)

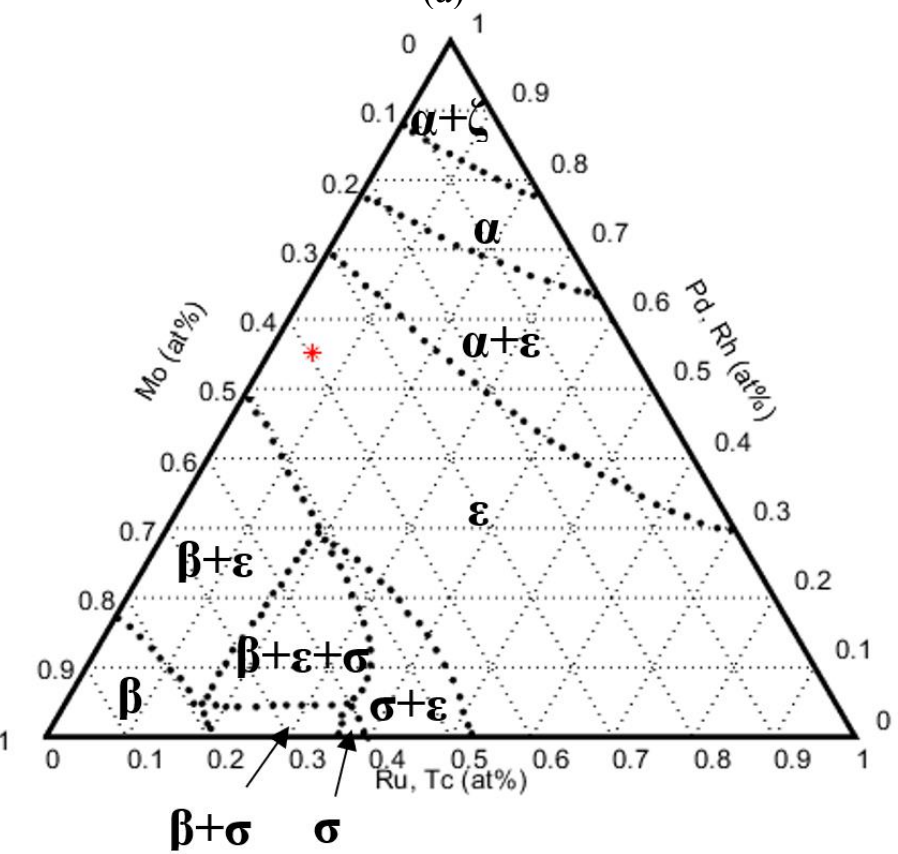

(b) 


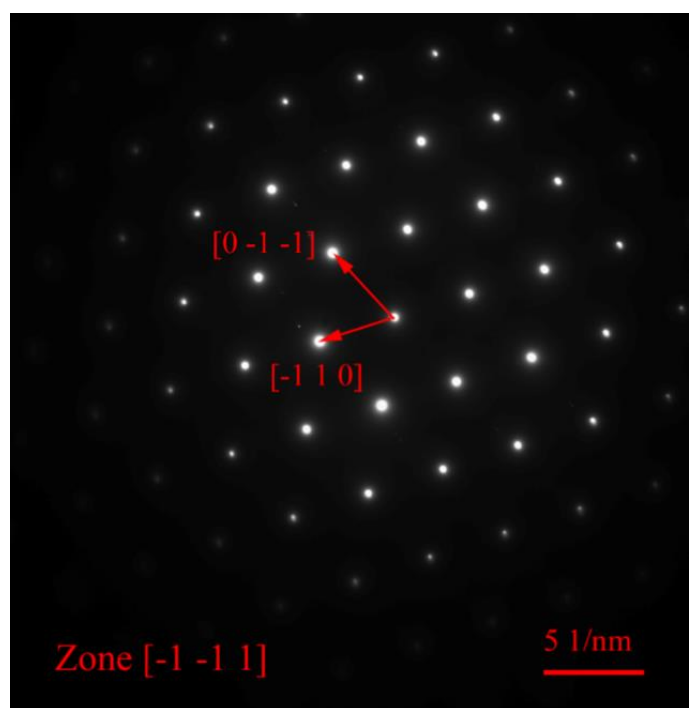

(c)

Figure 59. (a) EDS spectrum 1 from the metallic phase region. (b) Pseudo-ternary phase diagram of $\mathrm{Mo}(\mathrm{Pd}, \mathrm{Rh})(\mathrm{Ru}, \mathrm{Tc})$, modified from Ref. [7]. The red point represents the composition of the phase measured from the EDS spectrum in (a). (c) SAED pattern indexed according to the hexagonal phase predicted in the phase diagram.

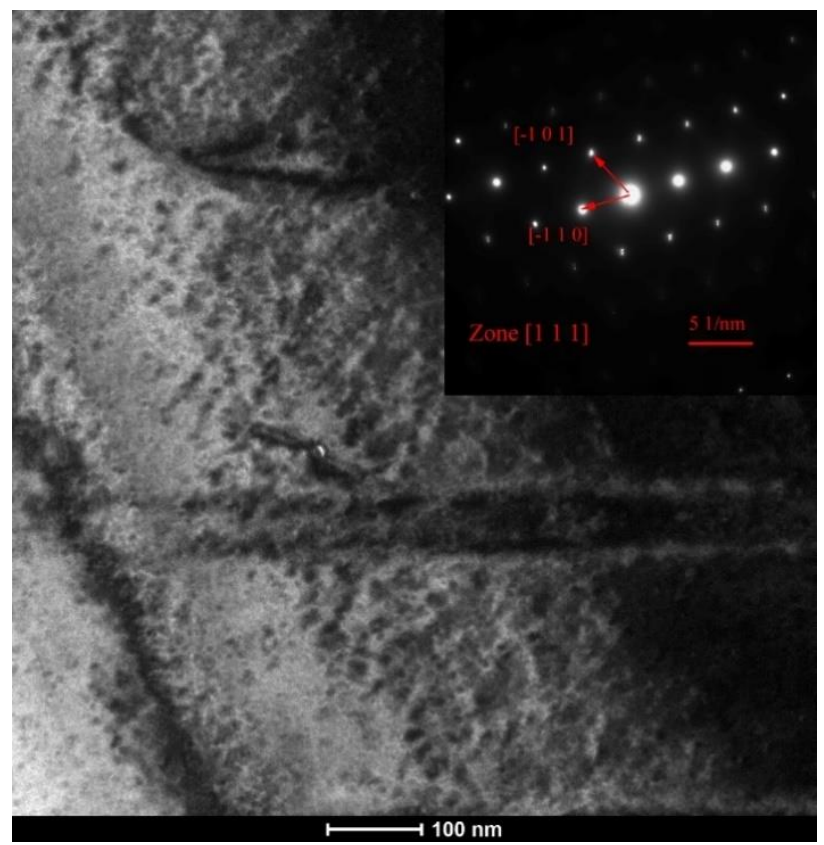

Figure 60. Twin in the FMP phase. 


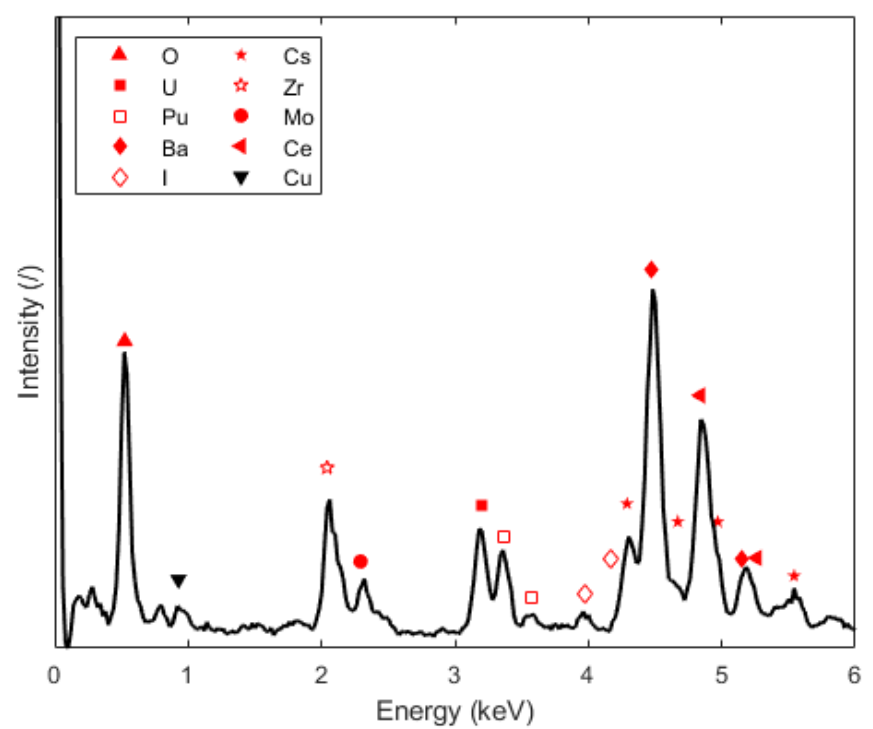

Figure 61. EDS spectrum from point 2 in Figure 58. 


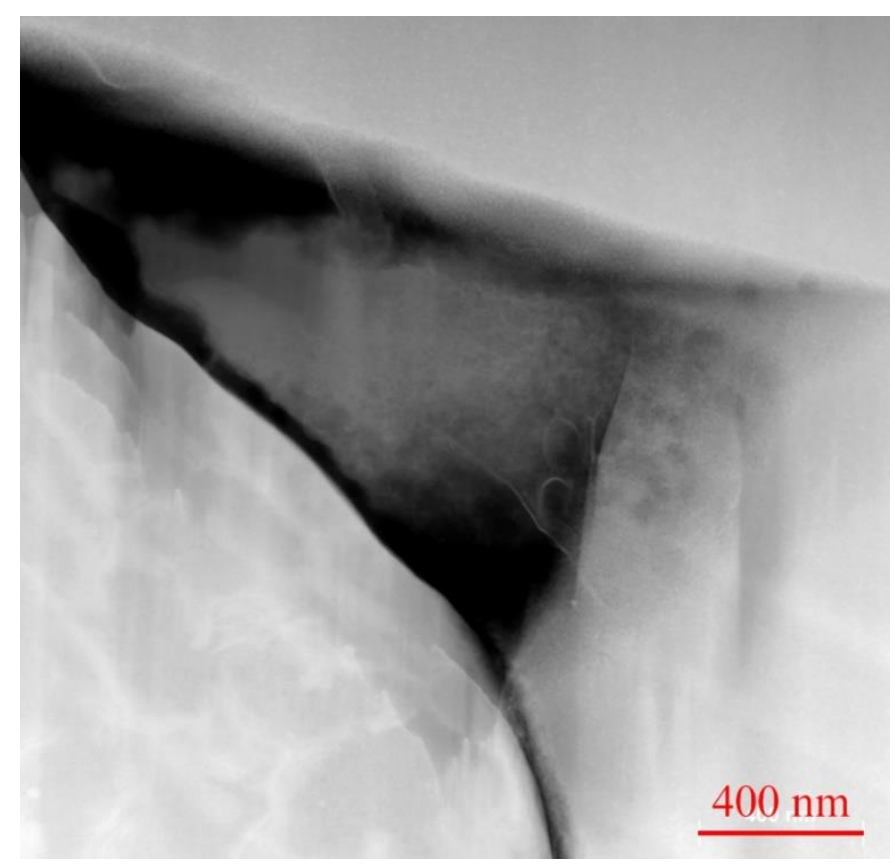

(a)

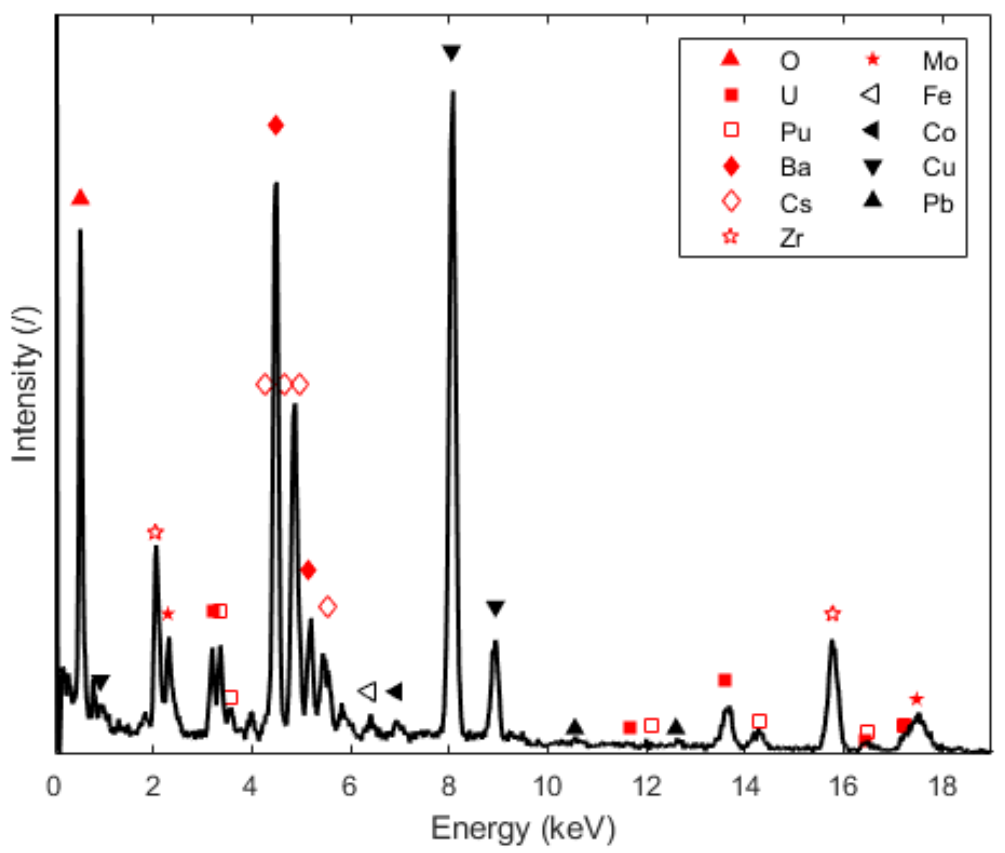

(b)

Figure 62. (a) Large grey phase inclusion at the interface between the MOX matrix and the FMP. (b) corresponding EDS spectrum. 


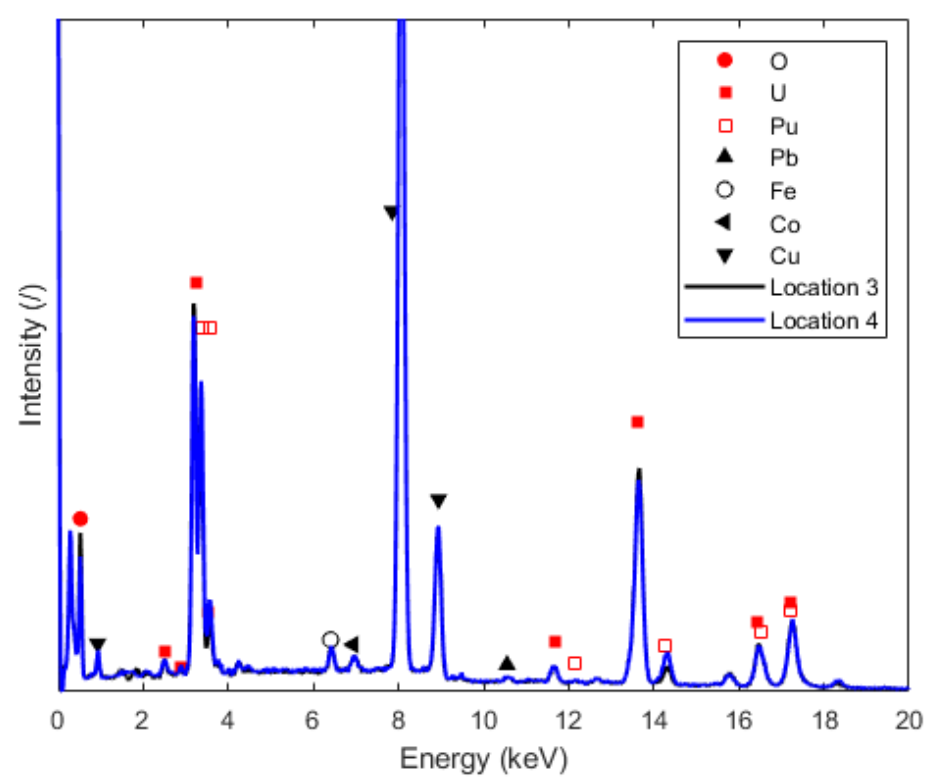

Figure 63. EDS spectra from the MOX phase.

Table 7. Uranium and plutonium quantification in the different grains of the MOX phase.

\begin{tabular}{|c|c|c|}
\hline Location & $\mathrm{U}$ (atomic \%) & $\mathrm{Pu}($ atomic $\%$ ) \\
\hline 3 & $85.24 \pm 17.02$ & $14.76 \pm 3.05$ \\
\hline 4 & $78.94 \pm 15.74$ & $21.06 \pm 4.33$ \\
\hline
\end{tabular}




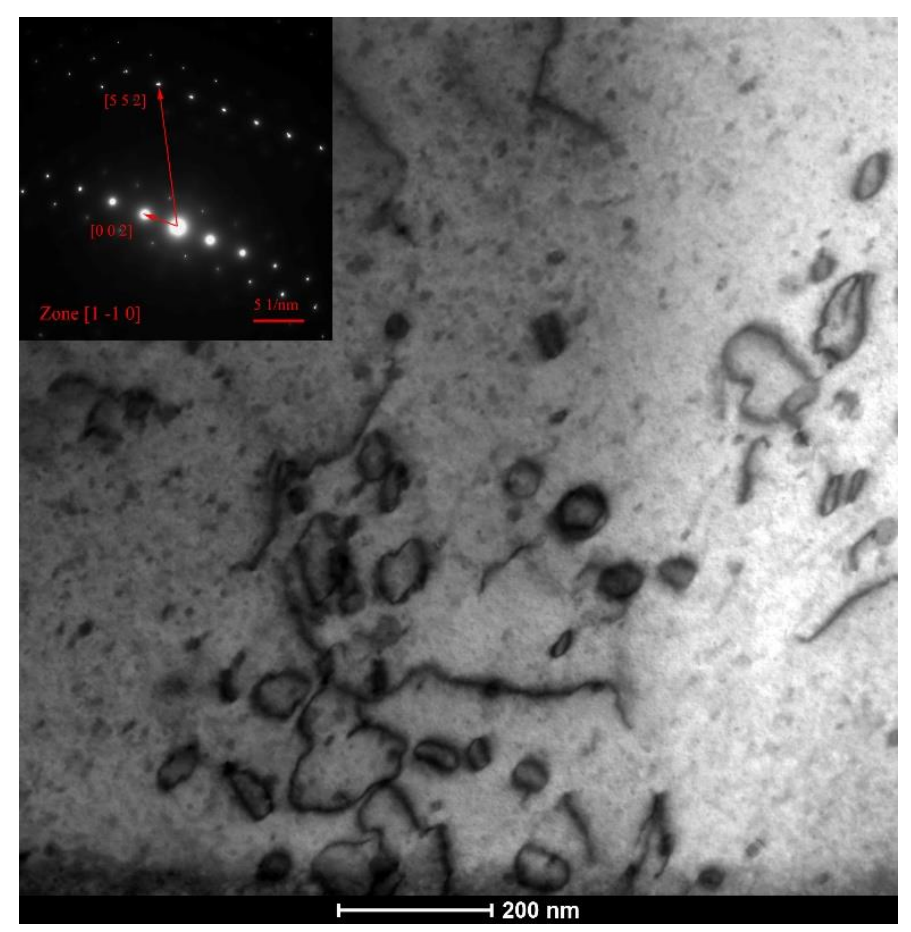

(a)

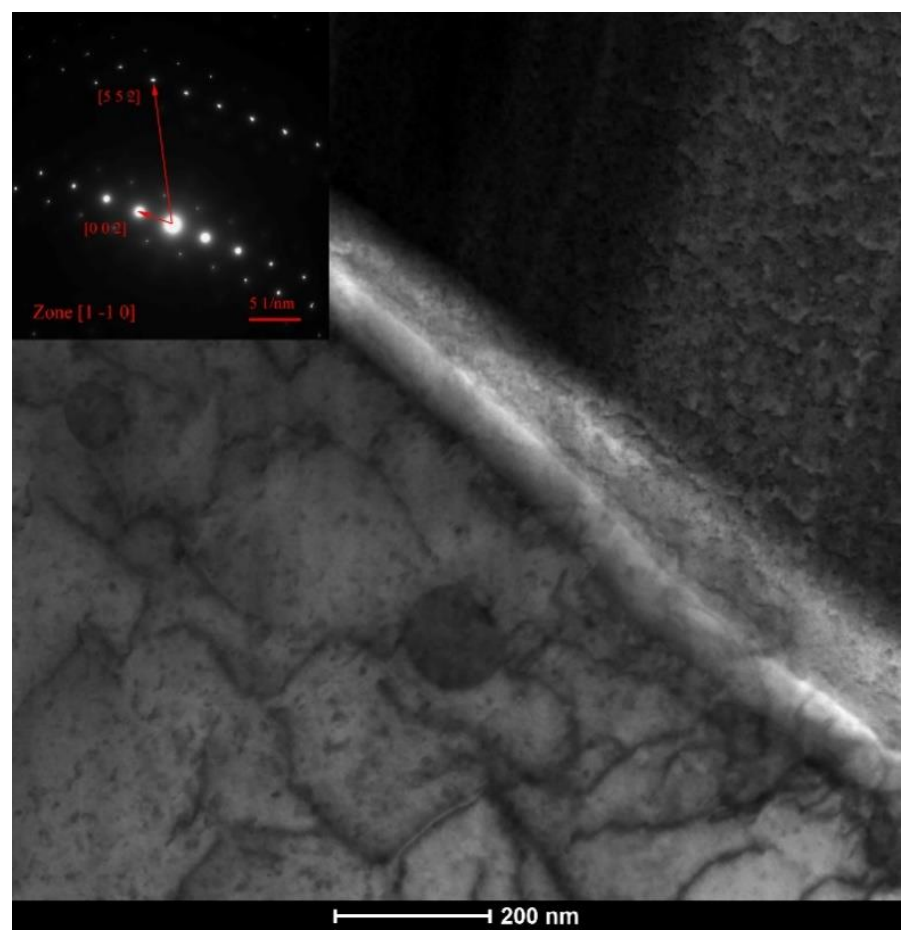

(b)

Figure 64. Images of the extended defects in the MOX phase. (a) dislocation loops, (b) dislocation lines pinned around the grey phase at the interface with the metallic phase. 


\subsubsection{Fuel-cladding chemical interaction}

The gap region of this sample was also examined to evaluate the onset of accumulation of fission products. The gap was open, but clear sign of contact between the fuel and the cladding with deposition of discontinuous layers of fission products was observed in various azimuthal locations. A large variety of locations have been investigated using EDS to gather qualitative data on the main components of the FCCI, followed by more accurate analyses using EPMA. Examples are displayed in Figure 65 through Figure 69. The morphology and composition of the FCCI was different from location to location. The migration of $\mathrm{Fe}$ and $\mathrm{Cr}$ towards the fuel varied not only from location to location, being more pronounced in area 3 (see Figure $67 \mathrm{~d}$ and e), but not in the other areas analyzed (see for example Figure 68e and f). Part of the cladding chemical species that have migrated to the fuel might be oxidized, as shown by presence of oxygen (Figure 67c and Figure 68d). Despite the local variations, common features could be observed in each location, which are shared with the previous samples. First, a Pd layer or Pd deposit could be observed at the interface with the HT-9 cladding (Figure 65b, Figure 66b, Figure 69h, Figure 68g and Figure 69b), sometimes in conjunction with Mo (Figure $65 \mathrm{e}$ ) and $\mathrm{Ru}, \mathrm{Tc}, \mathrm{Rh}$ or Ag. Iodine was measured occasionally only in traces (Figure 67o). Traces of $\mathrm{U}$ and $\mathrm{Pu}$ could be observed also attached to the cladding, as shown in Figure $65 \mathrm{f}$. $\mathrm{Cs}$, in conjunction with $\mathrm{Ba}$, and Te deposits were commonly observed (Figure $65 \mathrm{c}$ and d, Figure 66c and $\mathrm{d}$, Figure $69 \mathrm{c}$ and d). In one location in particular, the Cs and Te deposits were considerable, filling almost the entire gap (see Figure 69a).

In order to complete the analyses at different length scales and get structural information as well, the FCCI deposits shown in Figure 69 were selected to determine the evolution of the FCCI components from the fuel side to the cladding side. Two lamellae were extracted from the locations indicated in Figure 69a by the blue boxes. 


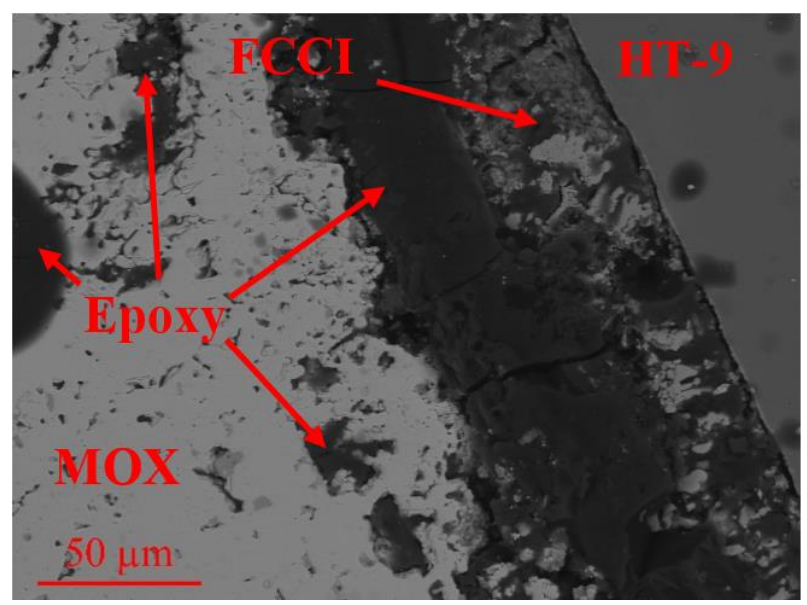

(a)

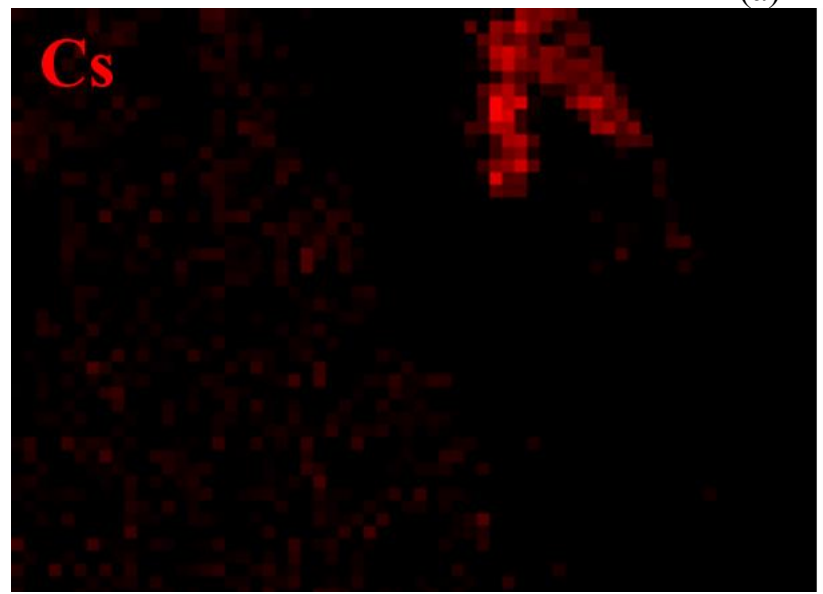

(c)

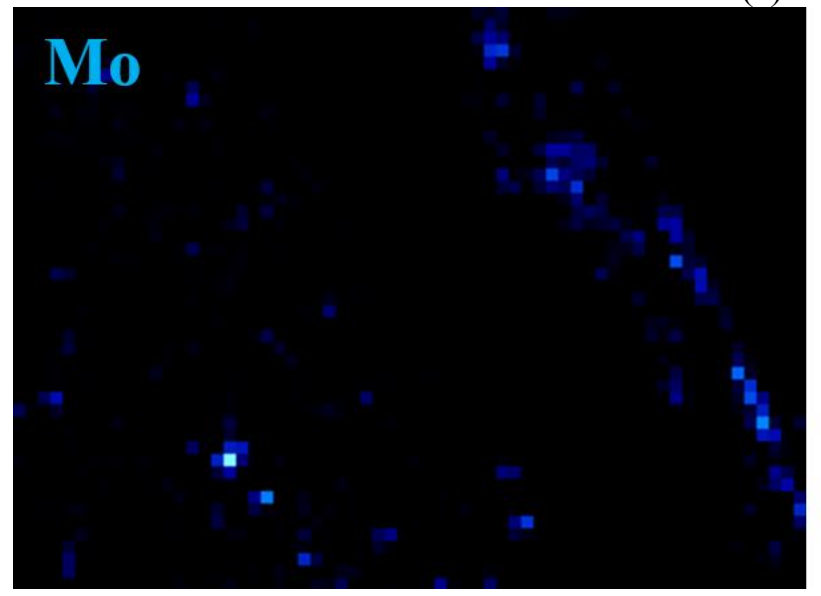

(e)

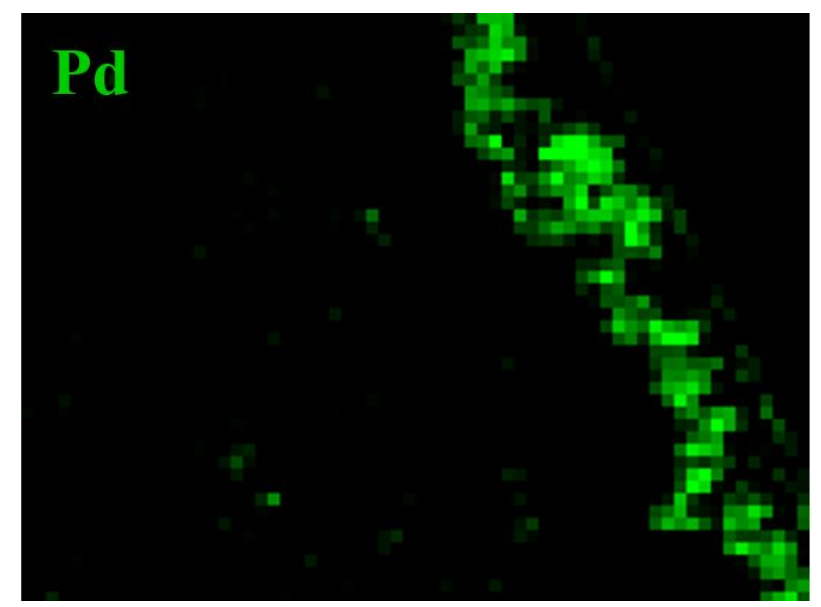

(b)
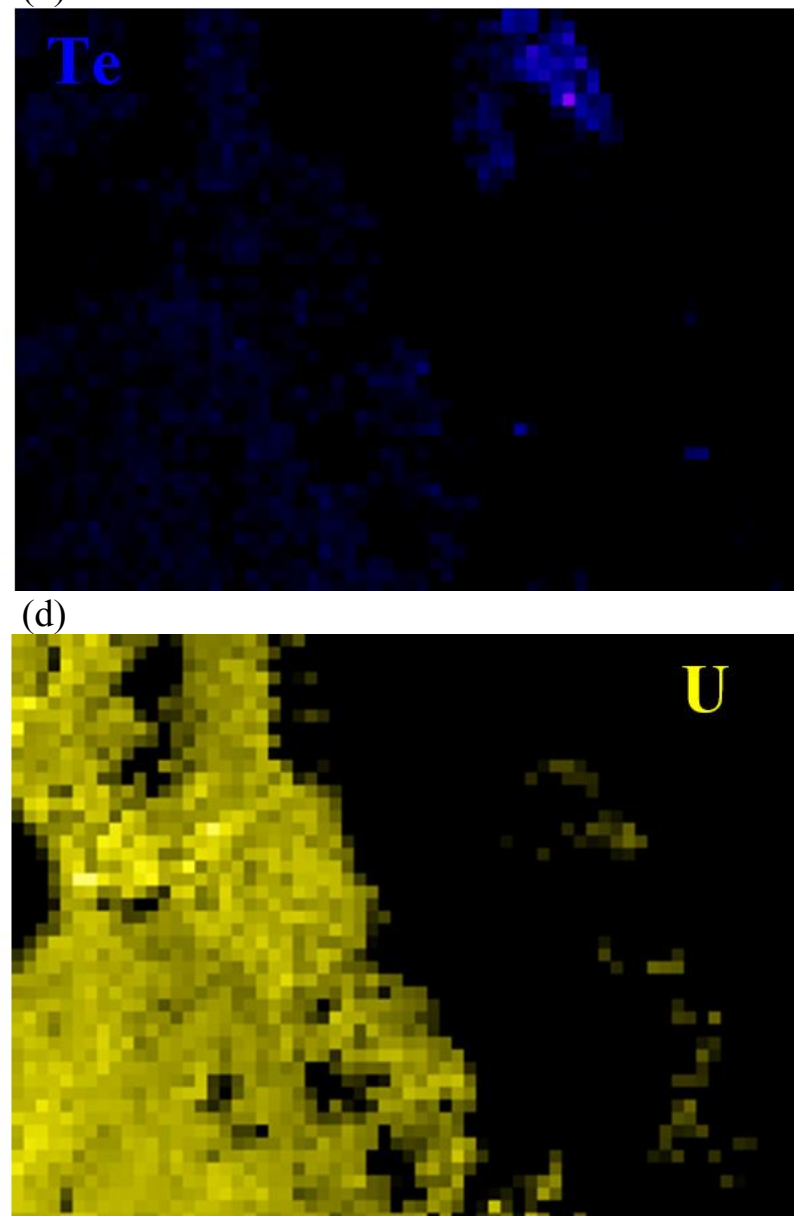

(f)

Figure 65. FCCI region. (a) BSE image, (b)-(e) most significant fission products, (f) U signal. 


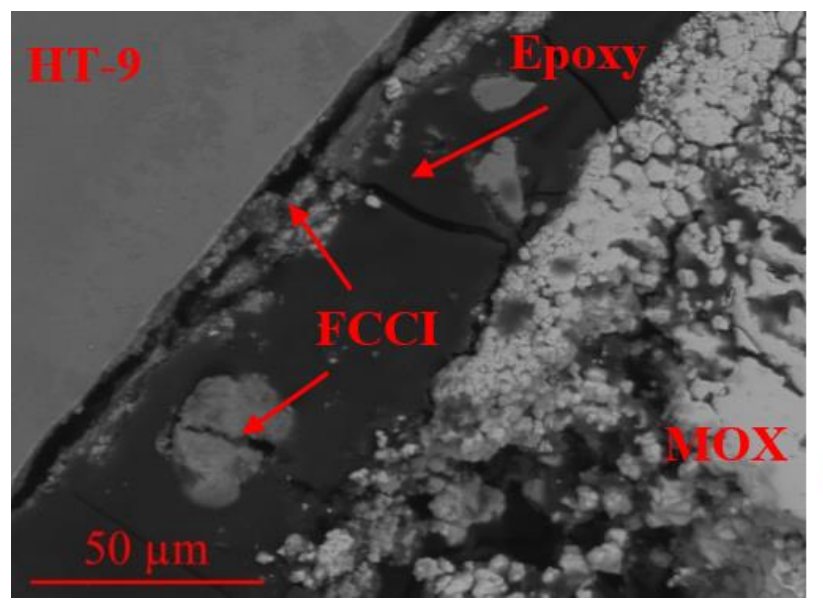

(a)

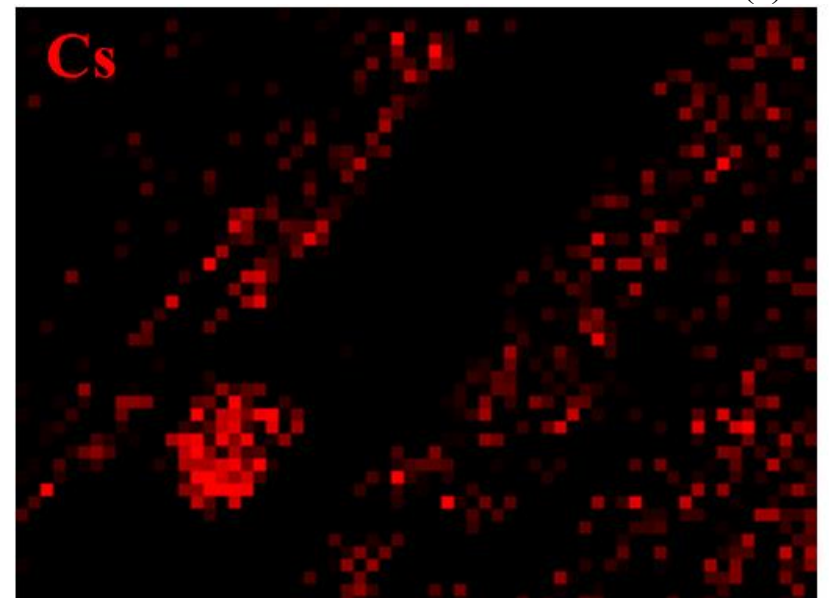

(c)

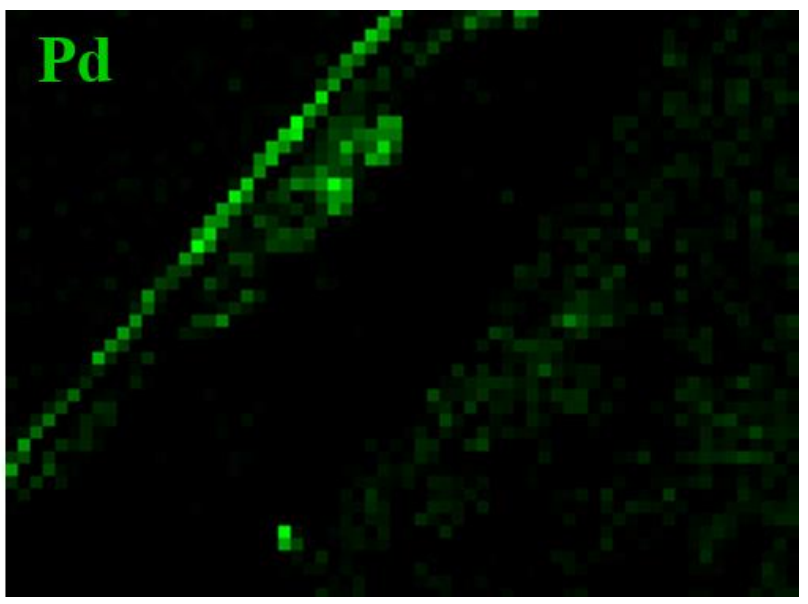

(b)

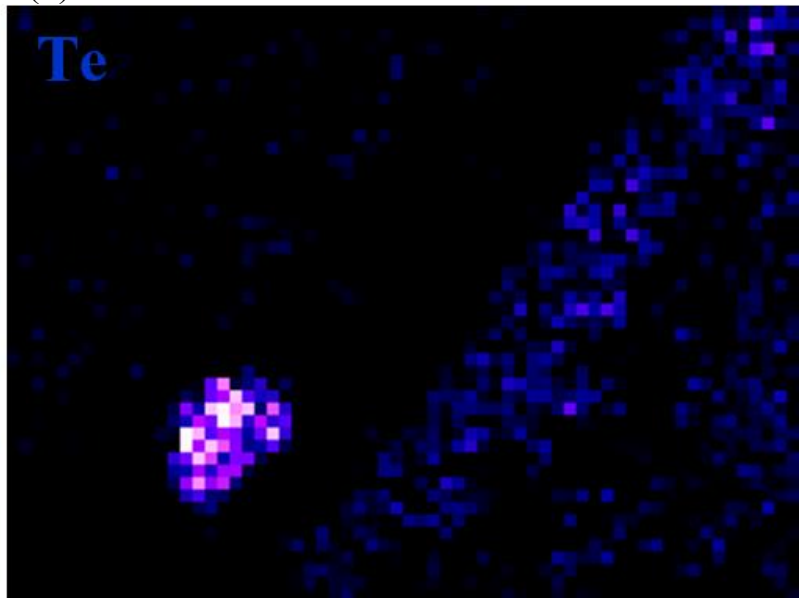

(d)

Figure 66. FCCI region. (a) BSE image, (b)-(d) most significant fission products. 
$\mathrm{U} \mathrm{At} \%$

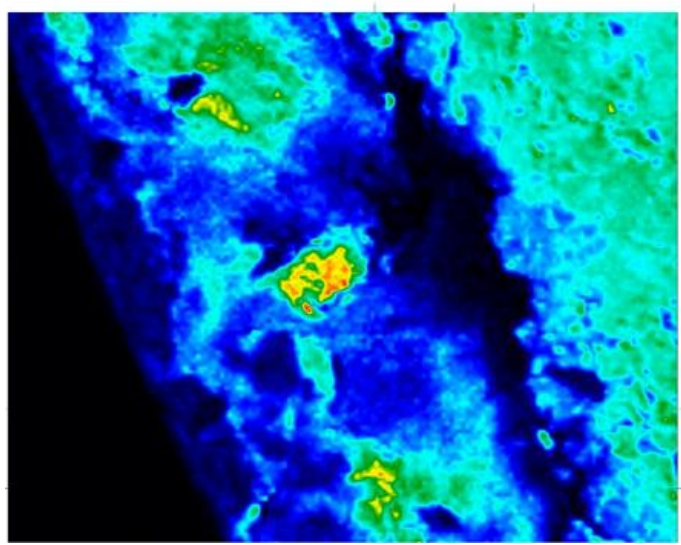

(a)

O At $\%$

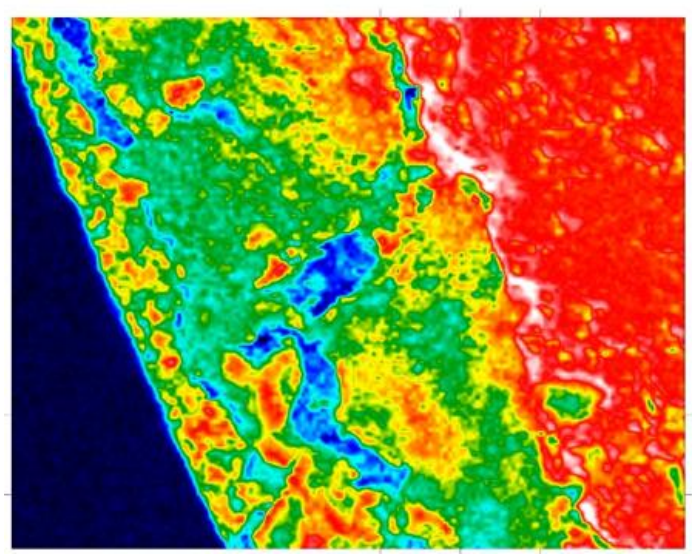

(c)

$\mathrm{Cr} A t \%$

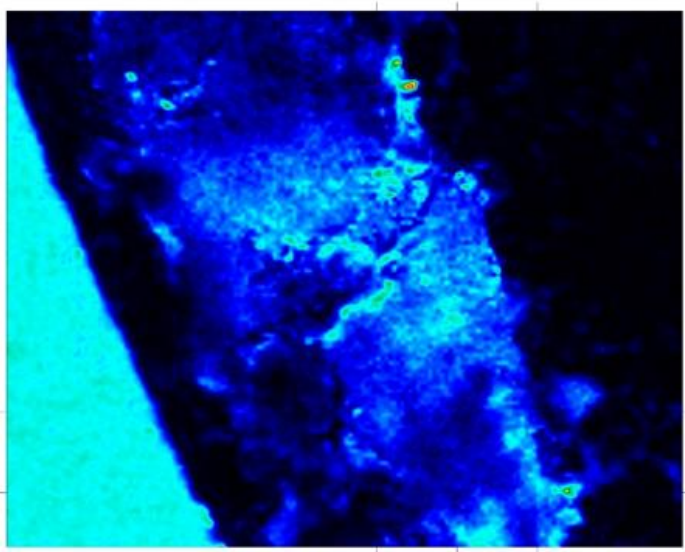

(e)
$\mathrm{Pu} \mathrm{At} \%$

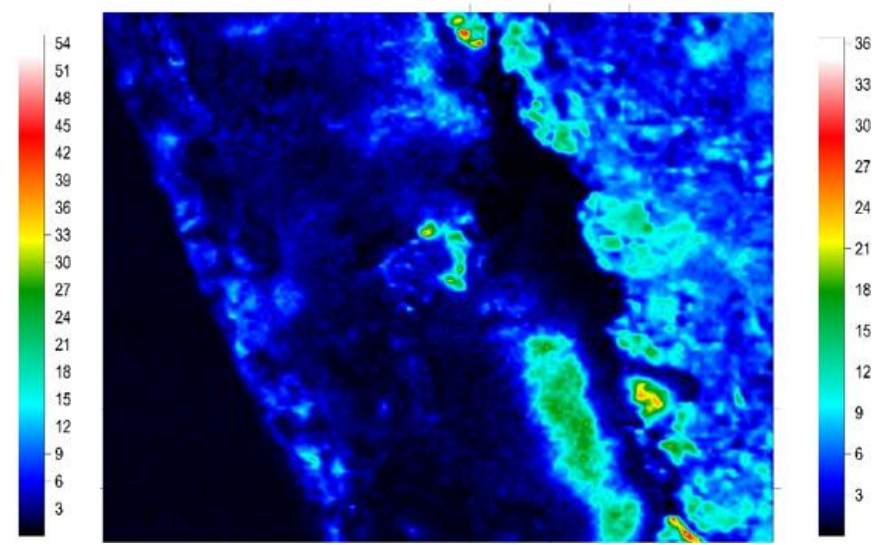

(b)

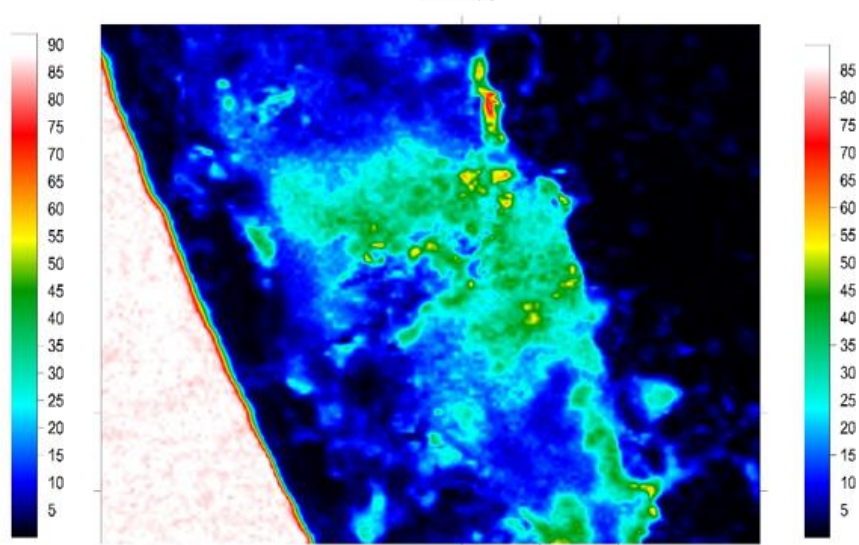

(d)

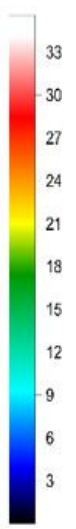

33
-30
27
24
21
18
15
12
-9
6
3

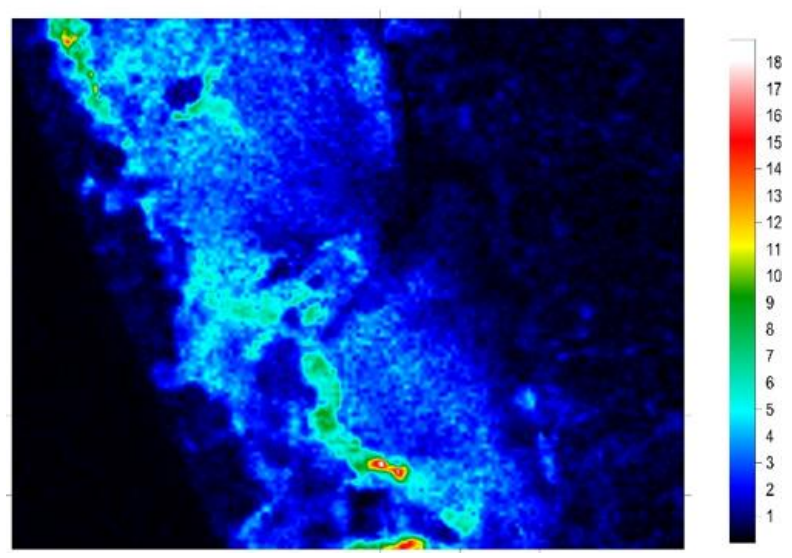

(f) 
Mo $\mathrm{At} \%$

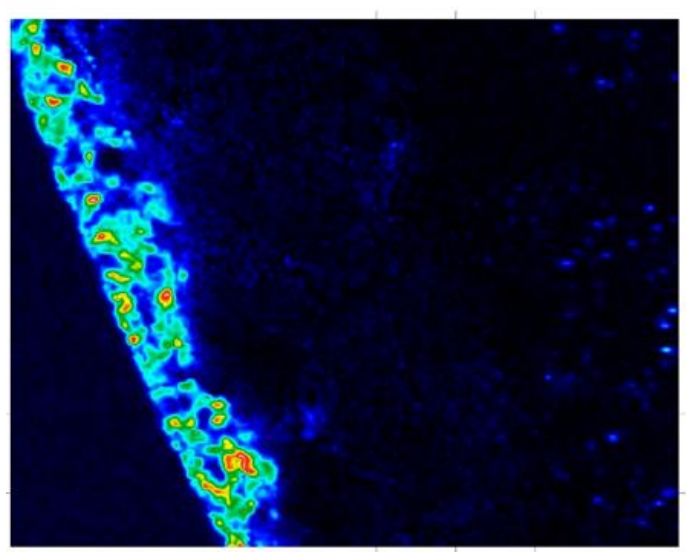

(g)

Te At $\%$

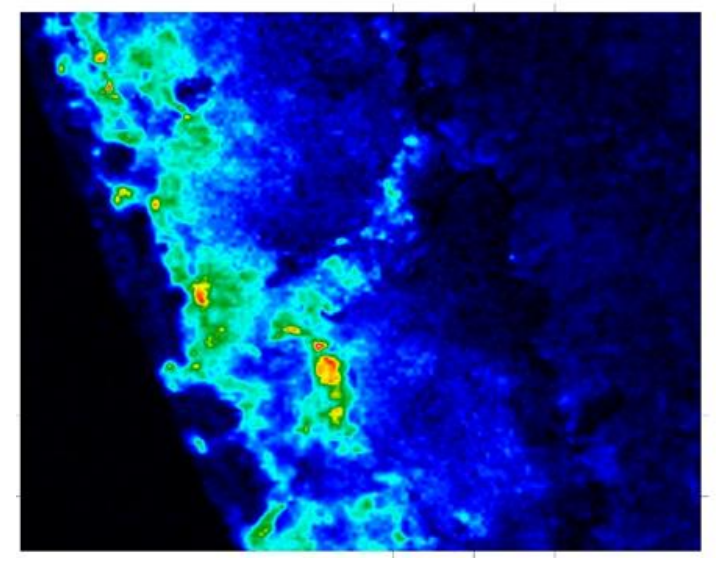

(i)

$\mathrm{Ag} \mathrm{At} \%$

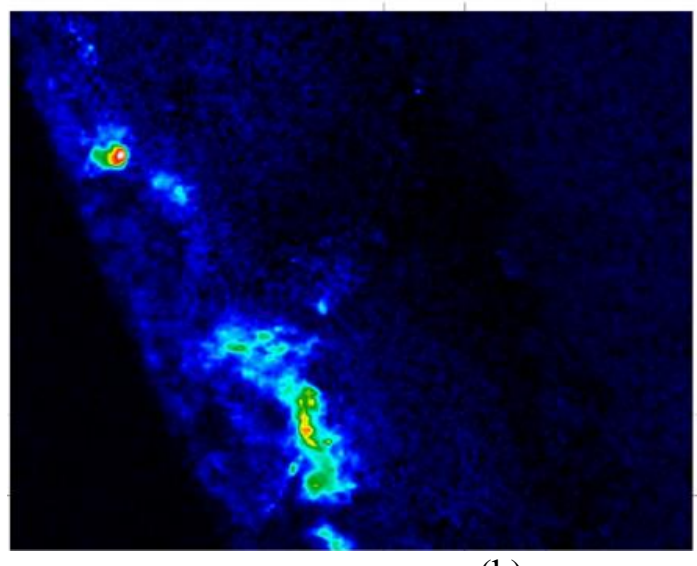

(k)
$\mathrm{Pd} \mathrm{At} \%$

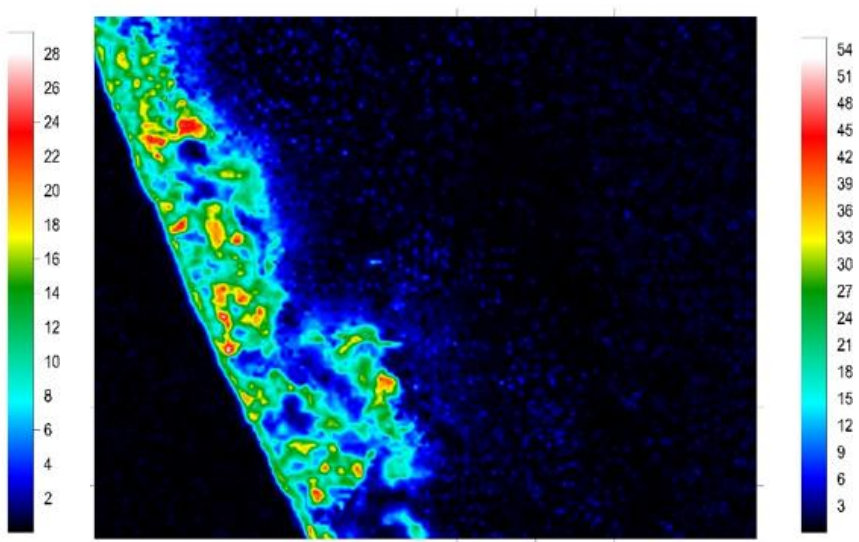

(h)

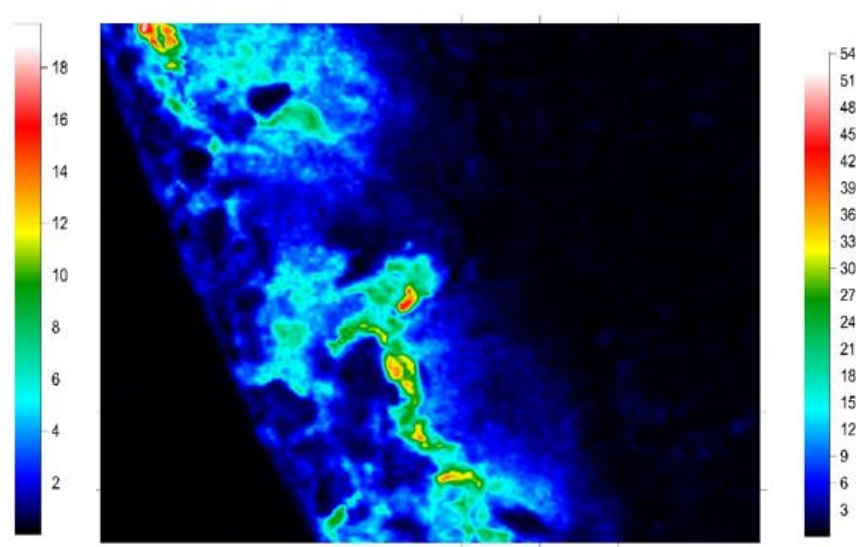

(j)

$\mathrm{Ru} A t \%$

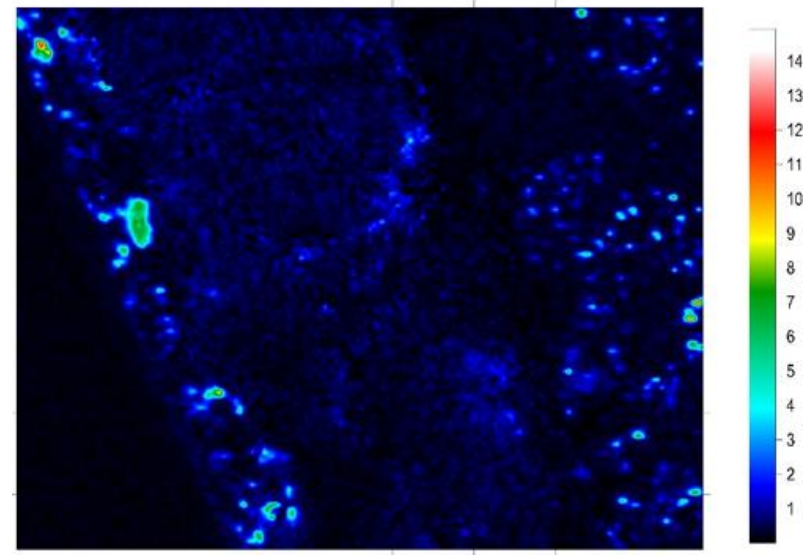

(1) 


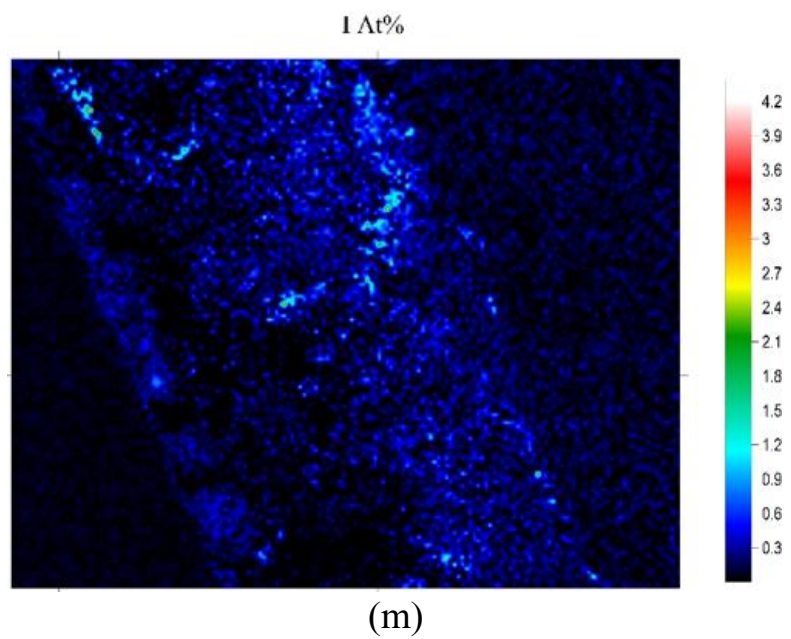

Figure 67. (a)-(m) Atomic maps of the FCCI region in area 3. 


\section{U At $\%$}

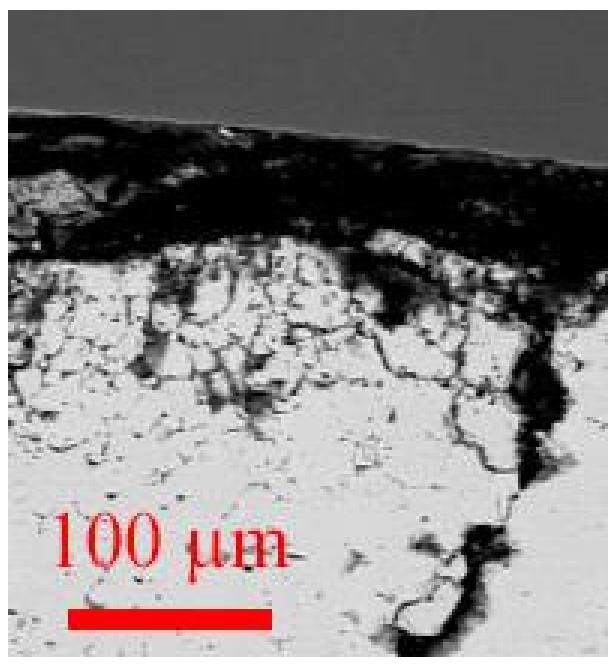

(a)

$\mathrm{Pu} \mathrm{At} \%$

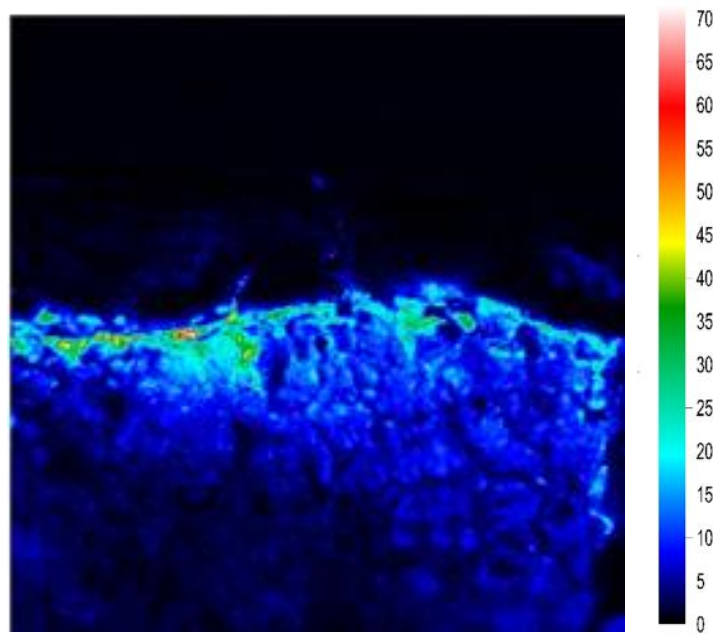

(c)

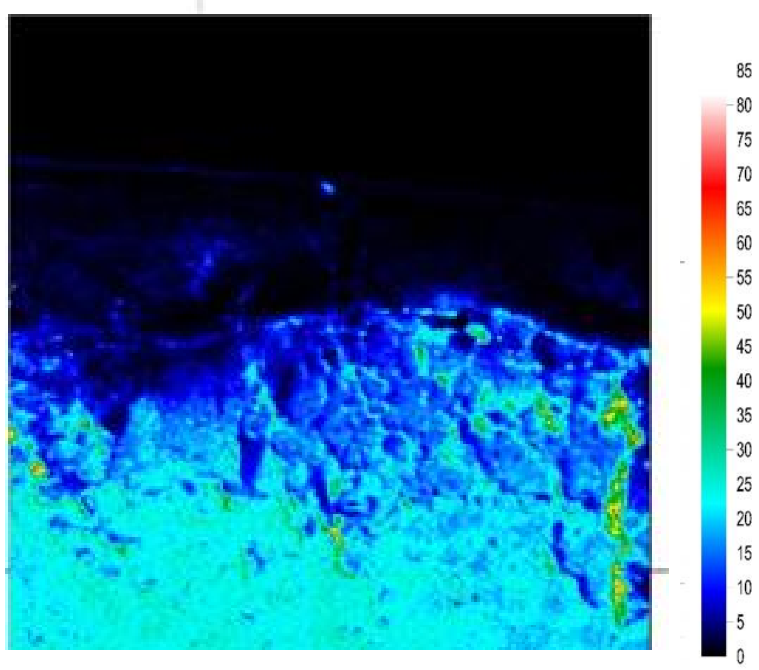

(b)

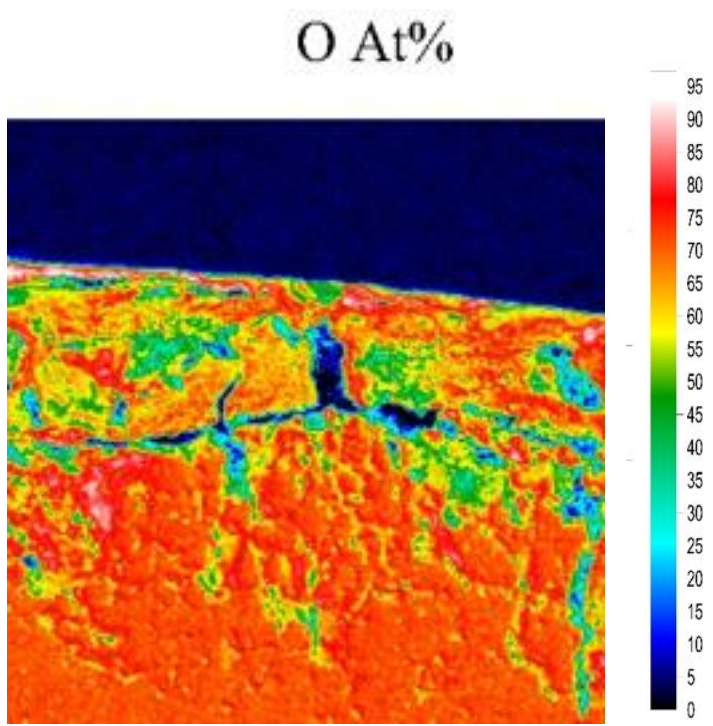

(d) 
$\mathrm{Fe} \mathrm{At} \%$

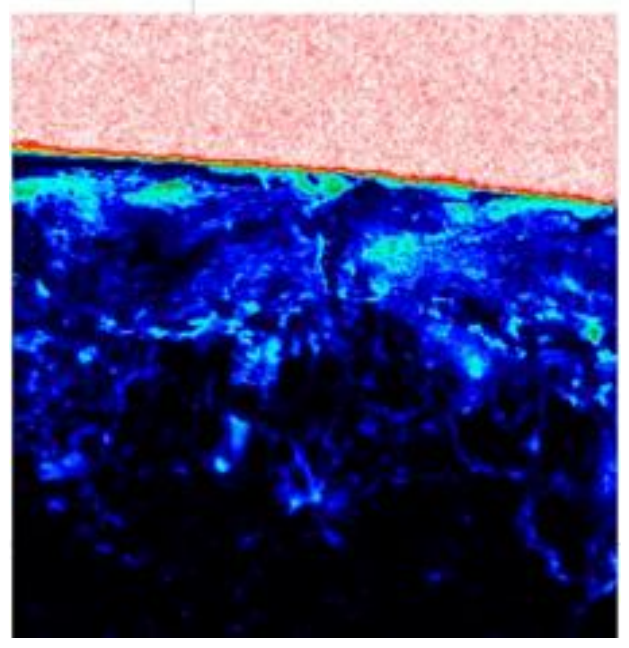

(e)

$\mathrm{Pd} \mathrm{At} \%$

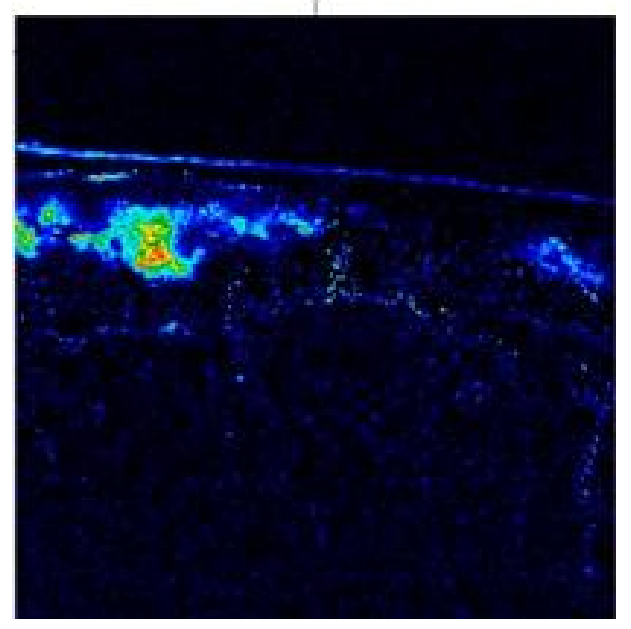

(g)
$\mathrm{Cr} \mathrm{At} \%$
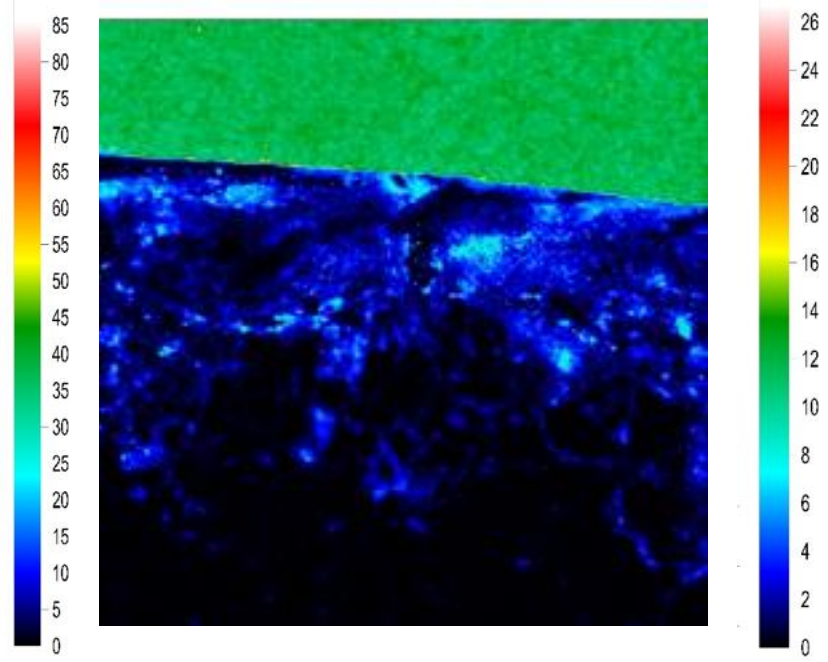

(f)

$\mathrm{Te} \mathrm{At} \%$

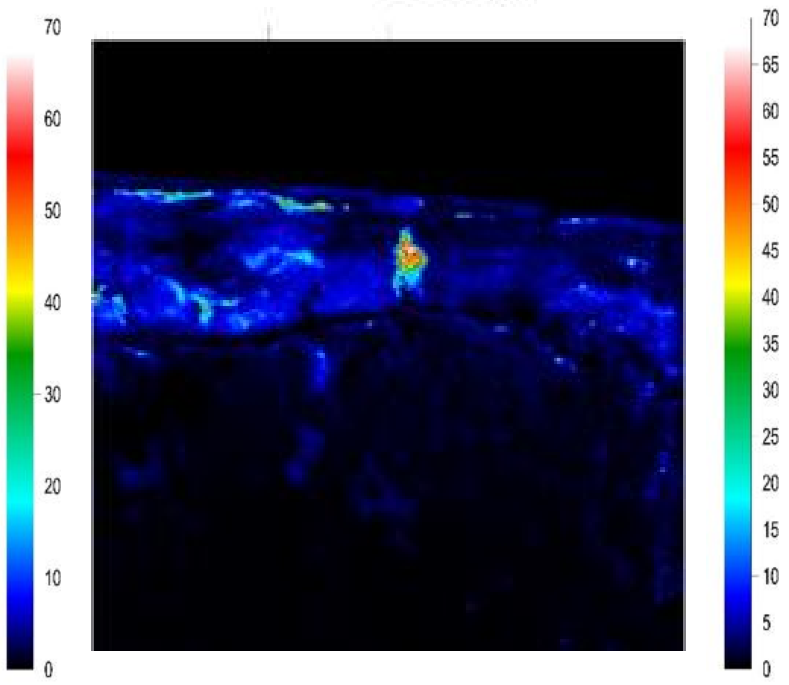

(h) 


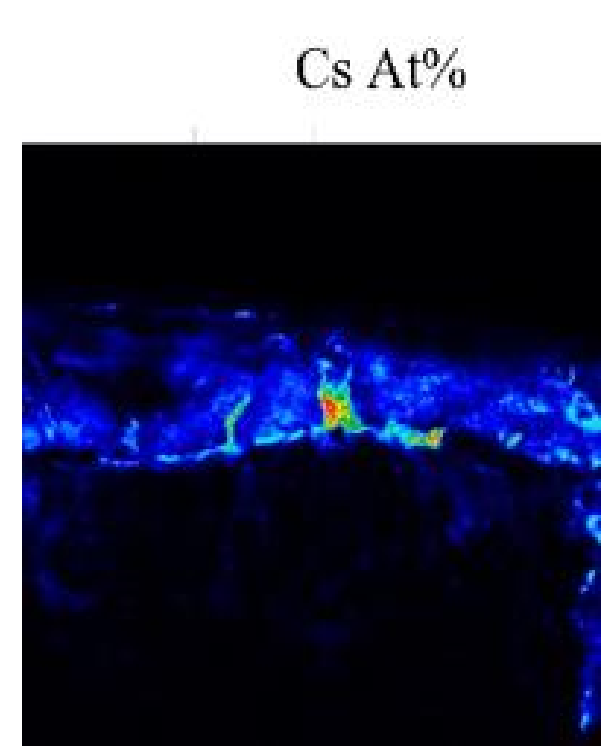

(i)

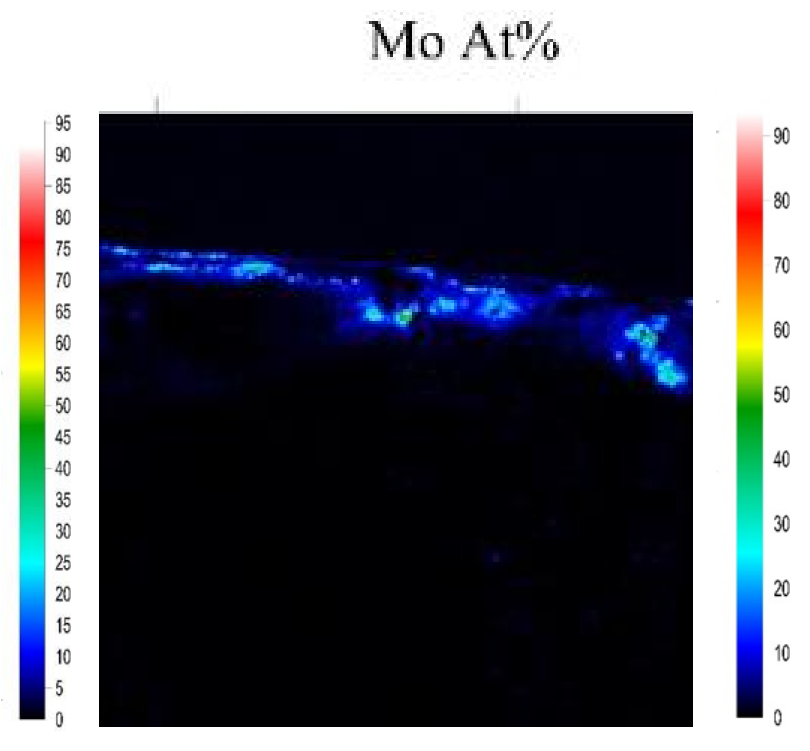

(1)

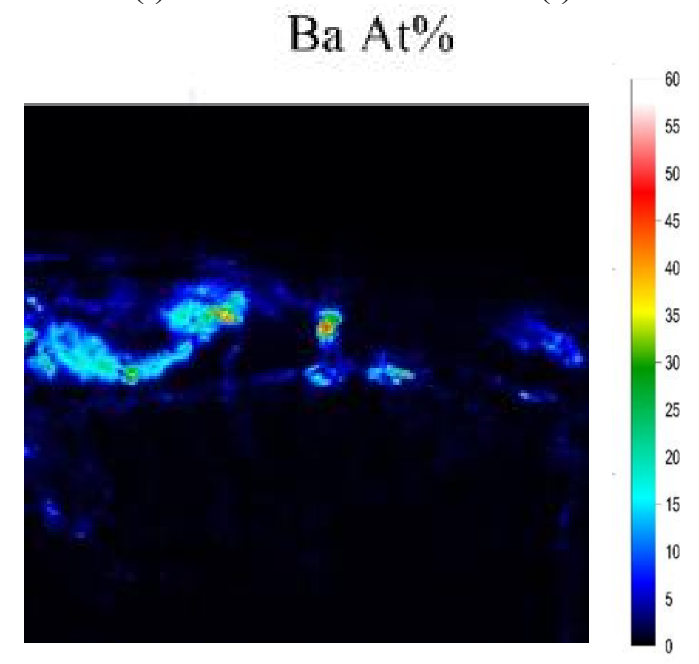

(m)

Figure 68. (a) BSE image of the FCCI from area 3 in Figure 51. (b)-(m) Atomic maps obtained with the EPMA. 


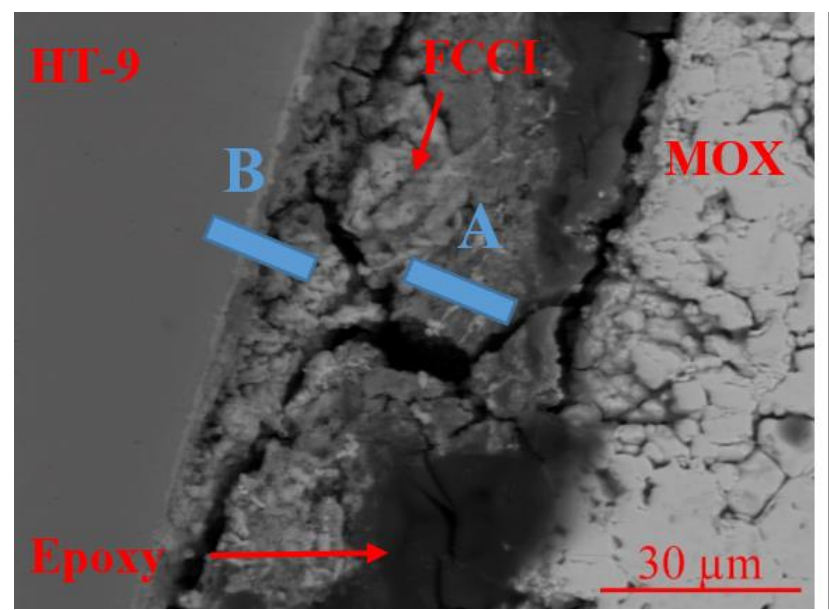

(a)

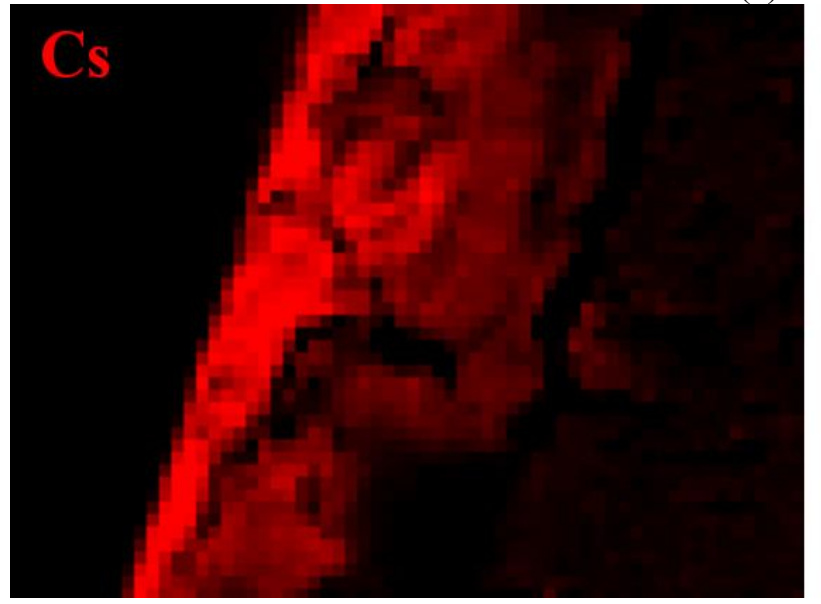

(c)

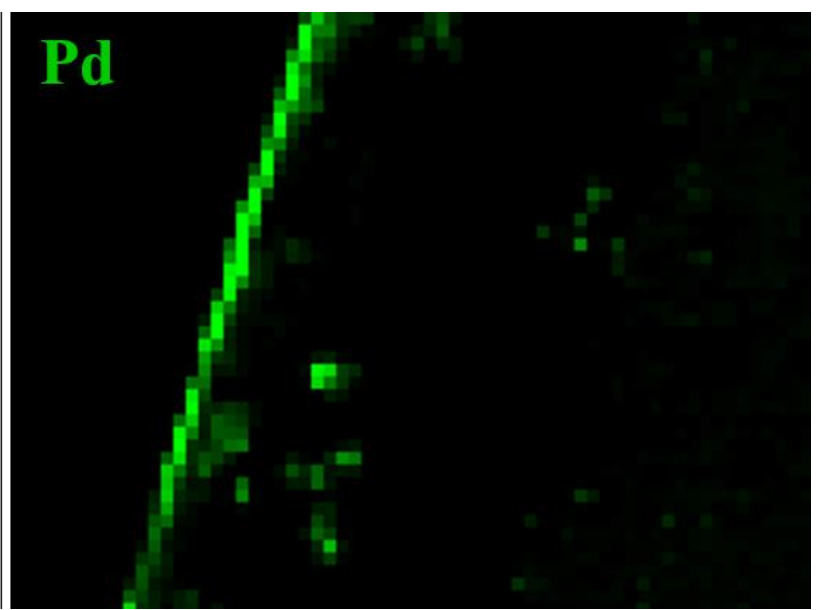

(b)

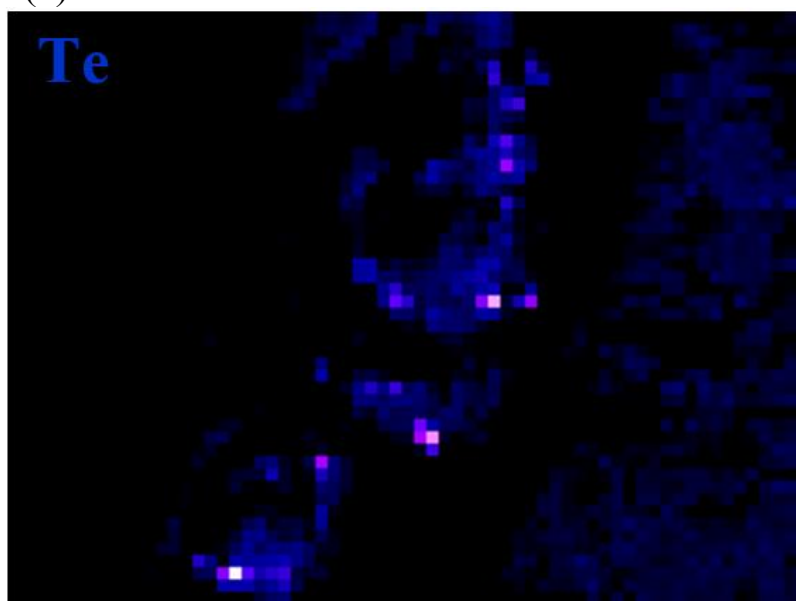

(d)

Figure 69. Higher magnification image of a thick portion of the FCCI in area 3. (a) BSE image with lamellae locations, (b)-(d) Most significant fission products (qualitative intensity maps).

The overview of lamella A is reported in Figure 70. Most of the lamella is formed by the epoxy used to prepare the sample for optical microscopy, which embedded nanocrystalline agglomerates. An enlarged view is shown in the next figure, Figure 71. The composition of the crystallites was different from spot to spot, comprising more than five fission products in traces, but, overall, three main fission products were predominant: Cs, Ba and Te. Maps showing their atomic distribution are reported in Figure 72. Each of the three major components segregates, forming agglomerates rich in one of the components. By comparing the maps in Figure $72 \mathrm{~b}$ and $\mathrm{c}$, it is clear that the Cs- and Ba-rich compounds are in the form of oxides, while the Te-rich nanoparticles contain Cs, but no oxygen. 


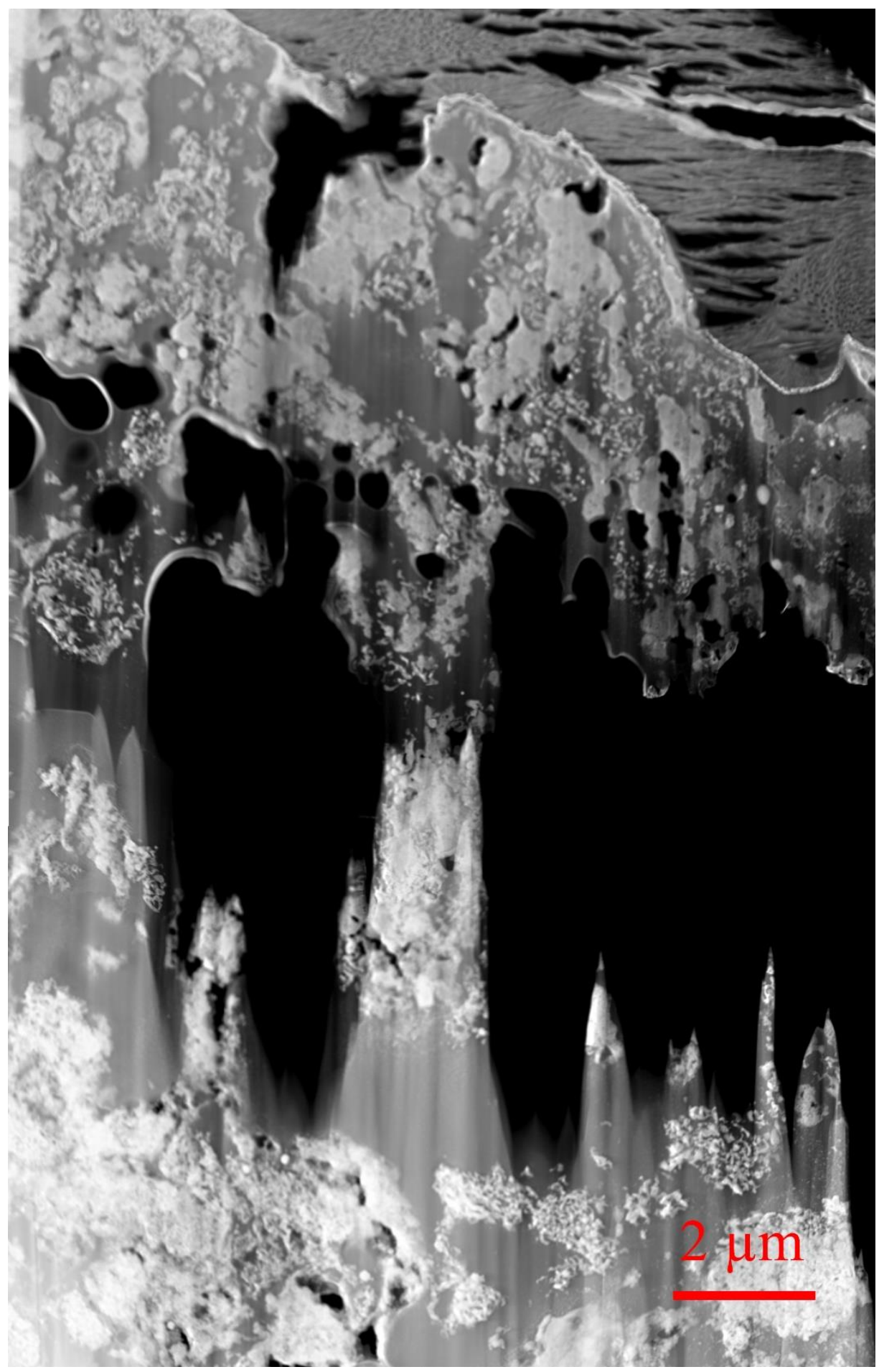

Figure 70. Overview of lamella A.

Quantification analyses were focused on each of the three main phases, which are summarized in Table 8. In addition to these three main phases, a spot rich in iodine was observed, enclosed in the black rectangle in Figure $72 \mathrm{~b}$. The composition showed a 36.08at $\% \pm 4.65$ at $\%$ of cesium, 34.62 at $\% \pm 2.98$ at $\%$ of iodine, 21.5 at $\% \pm 0.16$ at $\%$ of oxygen and 2.8 at $\% \pm 0.43$ at $\%$ of barium. 
The heterogeneous and nanocrystalline nature of the lamella made the diffraction analyses challenging. In the majority of the areas, multiple phases were simultaneously contributing to the collected SAED, resulting in patterns like the one shown in Figure 73b. Better patterns could be collected from isolated particles like the one shown in Figure 74a. The particle was tilted across multiple orientations, and the overlapping of two structures was obvious, as indicated by the circles in Figure $74 \mathrm{~b}$ and c. If the composition in Table 8 is compared to the Cs-Te phase diagram [32], Te and $\mathrm{CsTe}_{4}$ are predicted as the phases forming. Indeed, some diffraction patterns agreed extremely well with the trigonal structure of Te (e.g., Figure 74b), while, in another orientation, the monoclinic symmetry of $\mathrm{CsTe}_{4}$ gave the best fit to the pattern (Figure 74c). As for the Ba-rich phase, the region shown in Figure 75a was probed for crystallographic information. The ring pattern was checked against the structure of $\mathrm{CsBaO}_{3}$ (cubic, $\mathrm{Pm} \overline{3} \mathrm{~m}, \mathrm{n}^{\circ} 221$ ), $\mathrm{Cs}_{2} \mathrm{Ba}_{2} \mathrm{O}_{3}$ (monoclinic, $\mathrm{P} 2_{1} / \mathrm{c}, \mathrm{n}^{\circ} 14$ ) and a $\mathrm{BaUO}_{3}$ (orthorhombic, Pnma, $\mathrm{n}^{\circ} 62$ ). The high symmetric cubic system of $\mathrm{CsBaO}_{3}$ did not match any of the rings. The lower symmetric structure of $\mathrm{Cs}_{2} \mathrm{Ba}_{2} \mathrm{O}_{3}$ and $\mathrm{BaUO}_{3}$ did have signatures in the ring pattern (see Figure 75b). No significant crystallographic data could be collected from the Cs-rich phases.

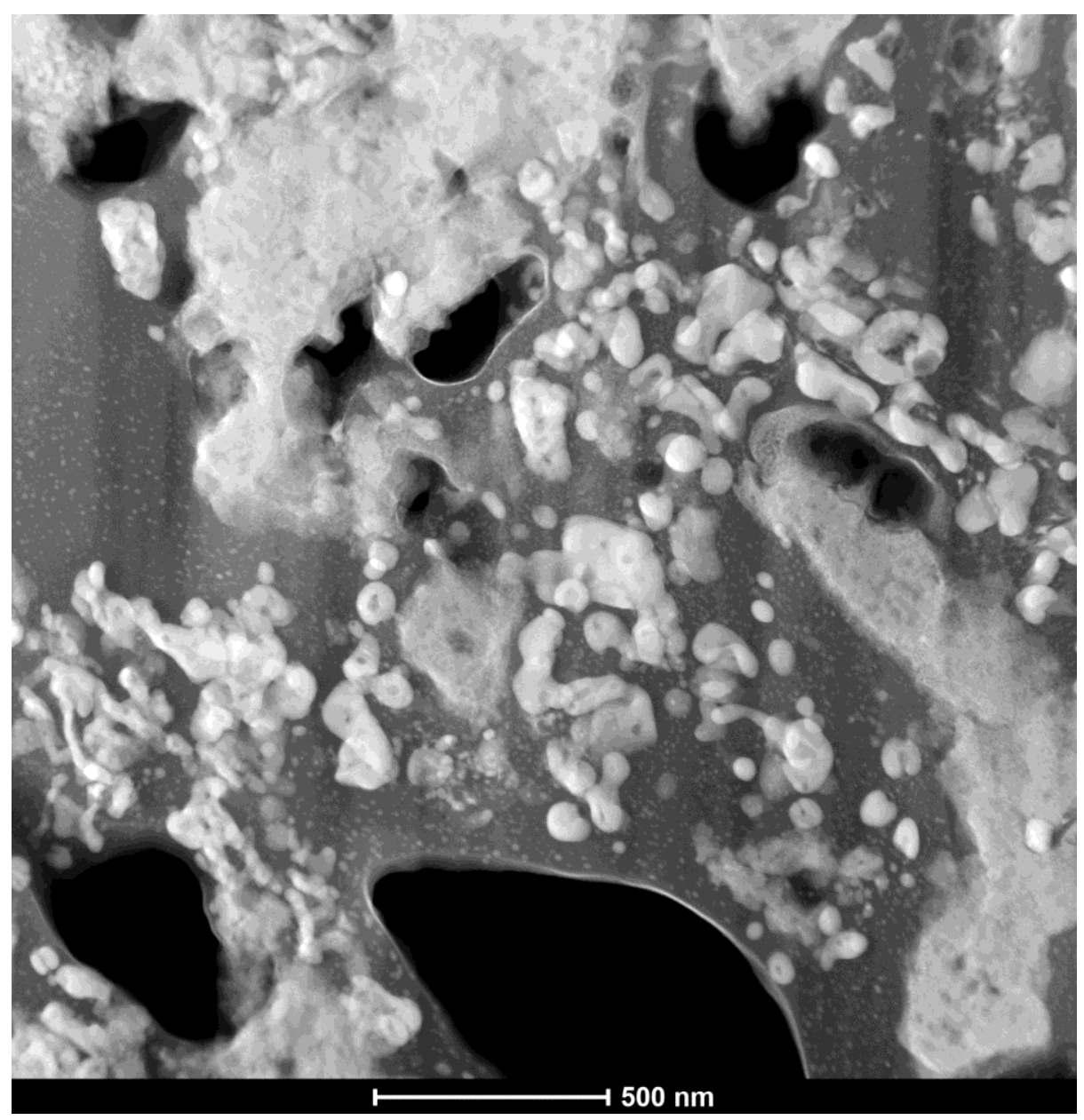

Figure 71. Enlarged view of the upper part of the lamella, highlighting the nano crystallites (white agglomerates) embedded in the epoxy matrix (mid-grey matrix). 


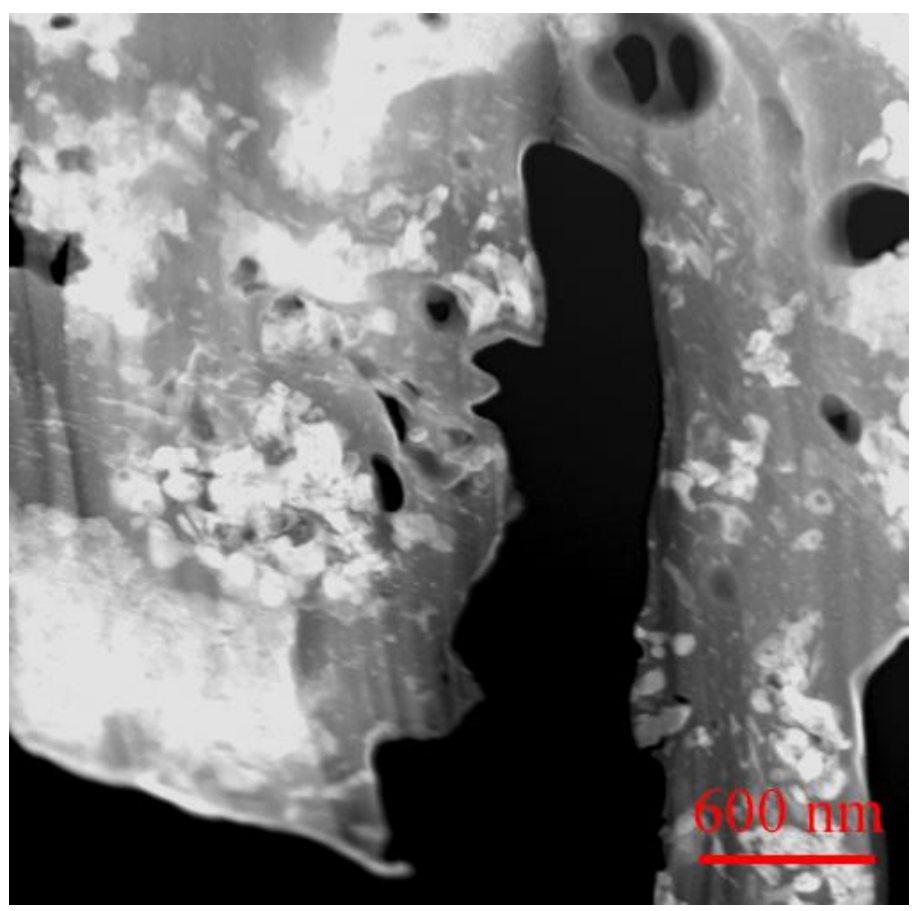

(a)

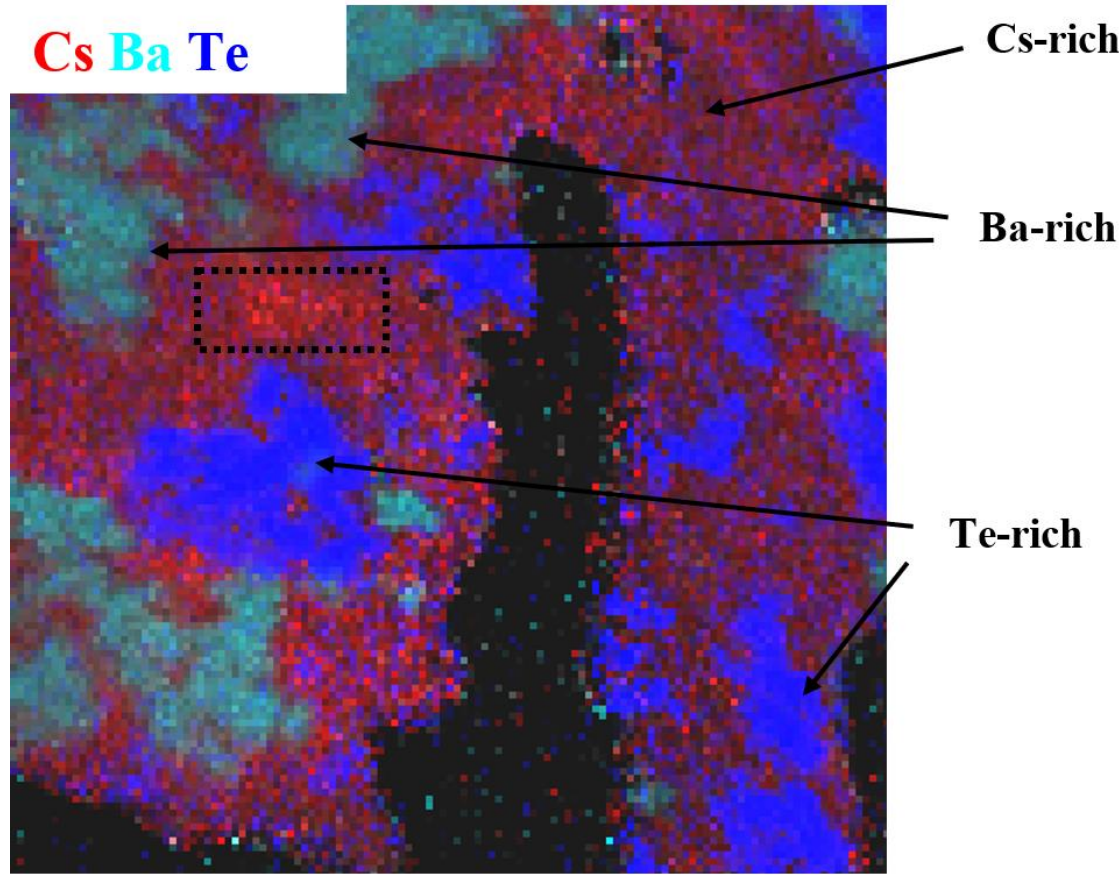

(b) 


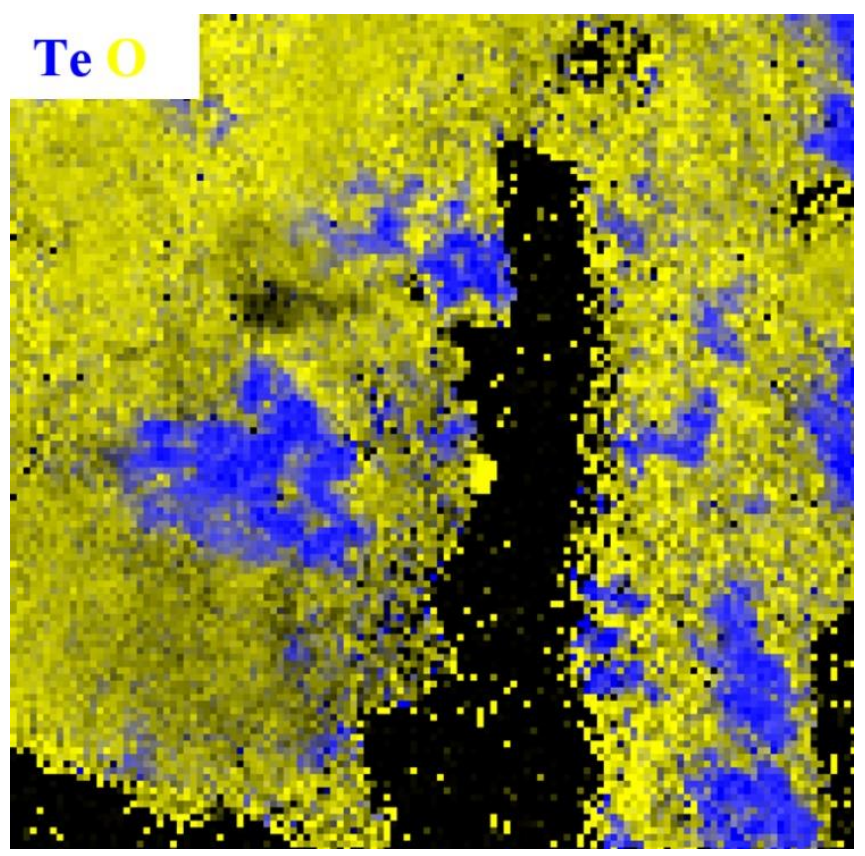

(c)

Figure 72. Distribution of the lamella's main components. (a) HAADF image, (b) EDS maps showing the at $\%$ of $\mathrm{Cs}, \mathrm{Ba}$ and Te. (c) Te and $\mathrm{O}$.

Table 8. Main components in the various phases in Figure $72 \mathrm{~b}$.

\begin{tabular}{lccc}
\hline & $\begin{array}{c}\text { Te-rich areas } \\
\mathbf{( a t \% )}\end{array}$ & $\begin{array}{c}\text { Cs-rich areas } \\
(\mathbf{a t \% )}\end{array}$ & $\begin{array}{c}\text { Ba-rich areas } \\
\text { (at\%) }\end{array}$ \\
\hline $\mathrm{Te}$ & $89.90 \pm 3.56$ & $13.74 \pm 1.90$ & $0.66 \pm 0.16$ \\
$\mathrm{Cs}$ & $6.26 \pm 0.69$ & $18.98 \pm 3.90$ & $17.48 \pm 2.6$ \\
$\mathrm{Ba}$ & $1.06 \pm 0.15$ & $1.75 \pm 0.42$ & $22.27 \pm 3.42$ \\
$\mathrm{O}$ & n.a. & $54.84 \pm 0.45$ & $42.41 \pm 0.25$ \\
$\mathrm{U}$ & n.a. & $0.43 \pm 0.35$ & $7.65 \pm 2.05$ \\
$\mathrm{Pu}$ & n.a. & $0.43 \pm 0.36$ & $0.55 \pm 0.18$ \\
$\mathrm{Mo}$ & $0.75 \pm 0.09$ & $4.80 \pm 0.41$ & $3.75 \pm 0.18$ \\
$\mathrm{Zr}$ & $1.50 \pm 0.11$ & $1.33 \pm 0.17$ & $1.77 \pm 0.10$ \\
$\mathrm{I}$ & n.a. & $1.73 \pm 0.68$ & $1.73 \pm 0.27$ \\
others & 0.53 & 3.7 & 1.73 \\
\hline
\end{tabular}



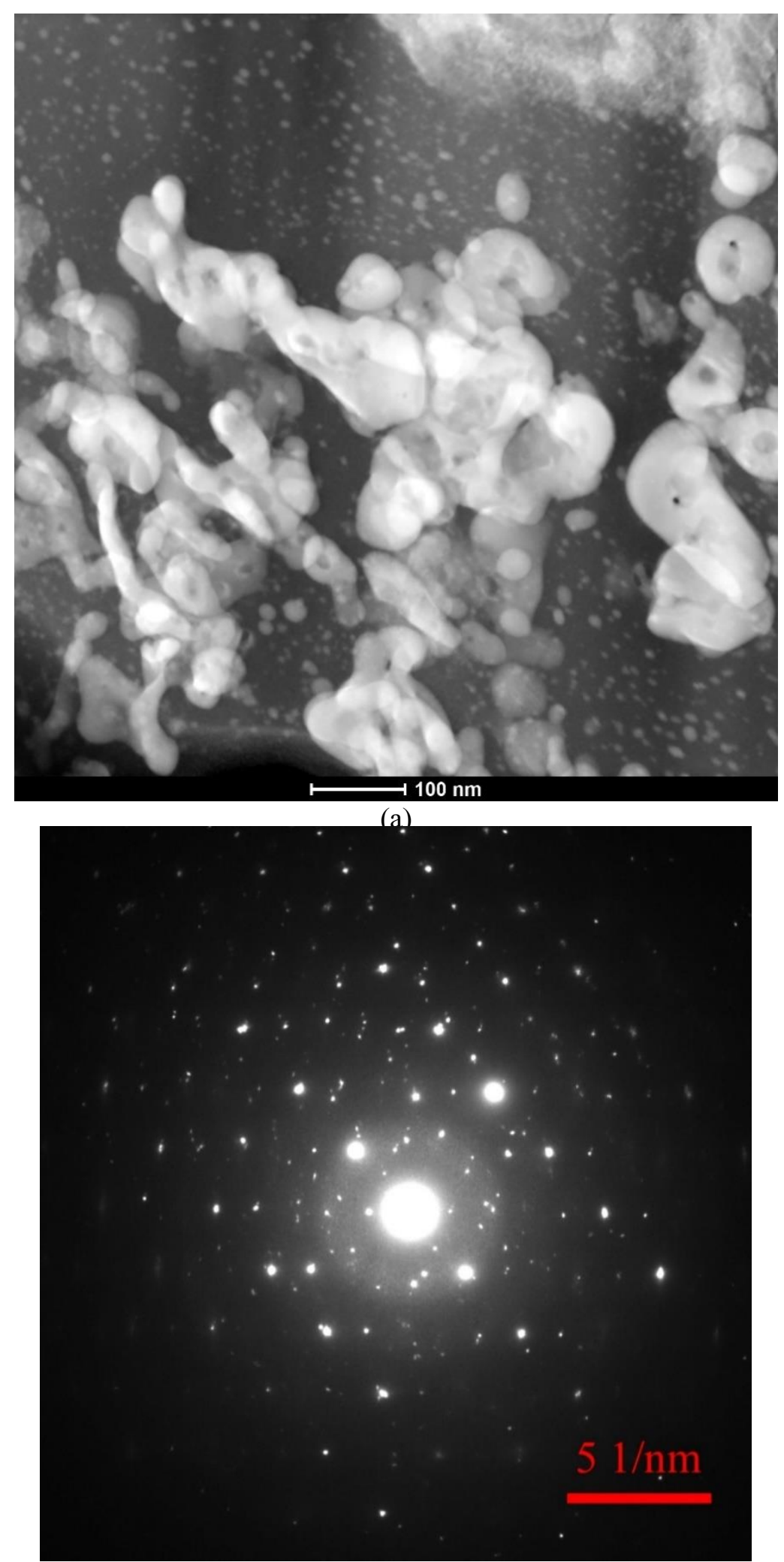

(b)

Figure 73. Typical morphology and diffraction pattern collected from the Te-rich phase. (a) HAADF image. (b) Corresponding SAED pattern. 


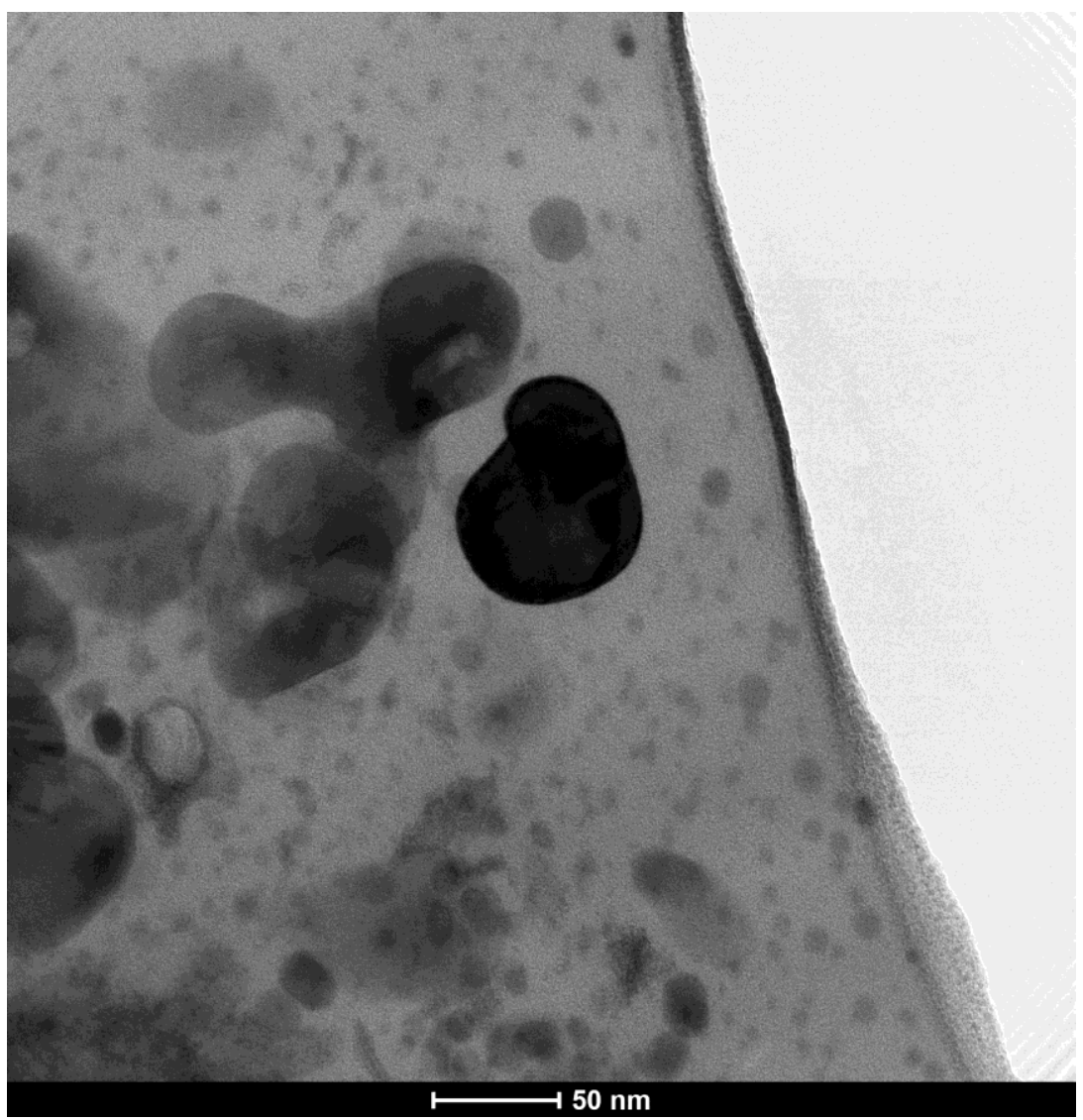

(a)

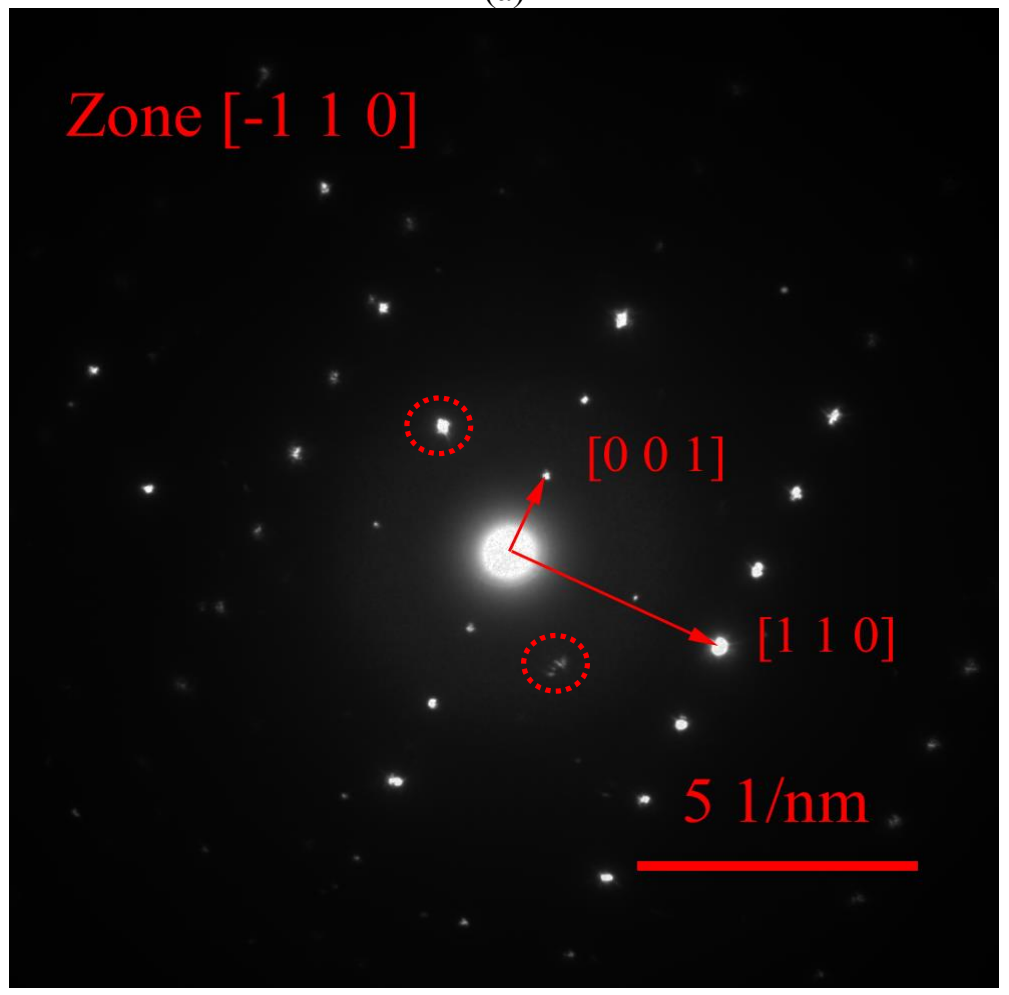

(b) 


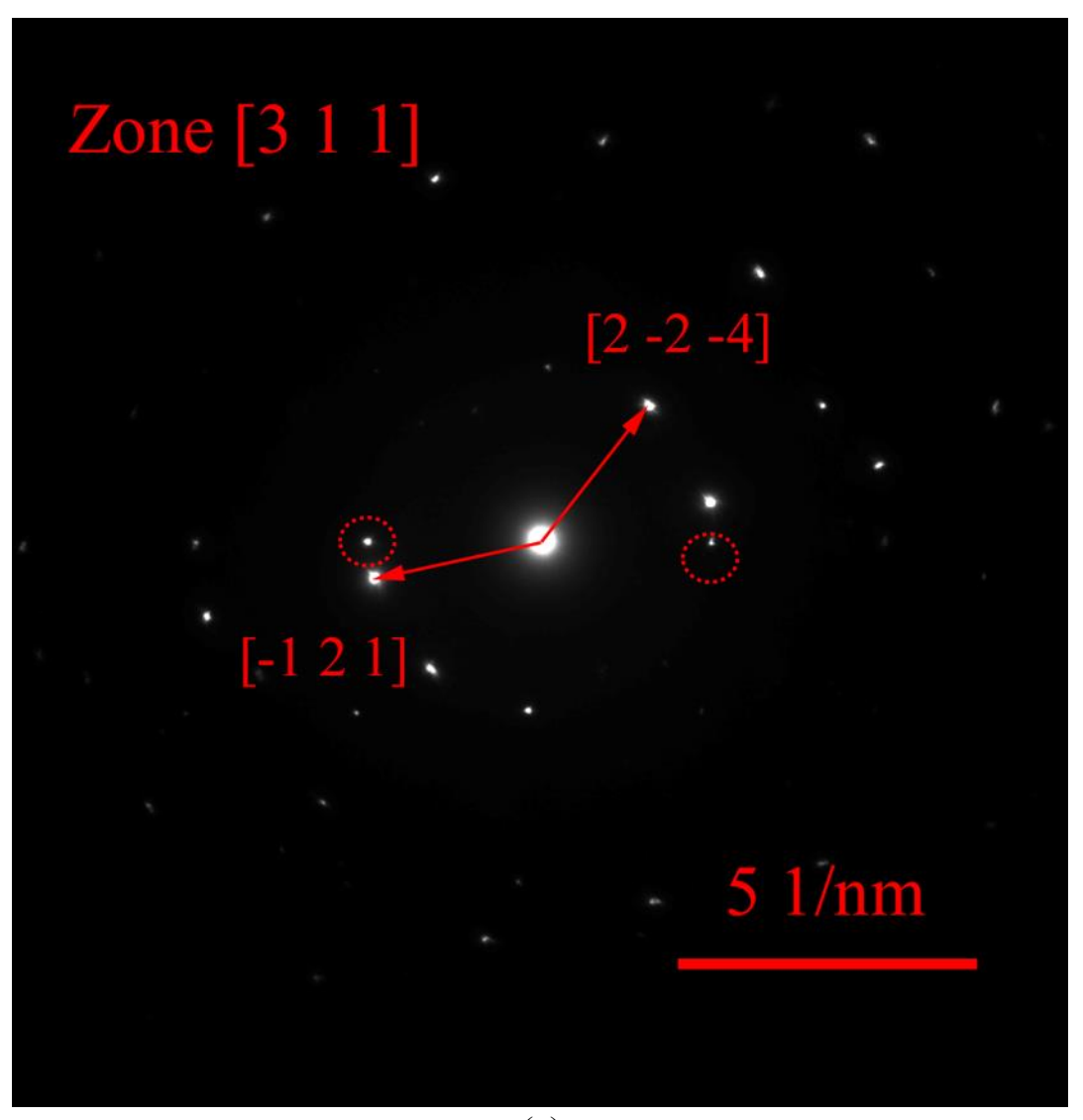

(c)

Figure 74. (a) Te-rich particle composed of two crystalline domains. (b) SAED pattern indexed by the pure Te structure. (c) SAED pattern indexed with the CsTe4 structure. In each of the patterns, reflections from a different crystallite are highlighted by the circles. 


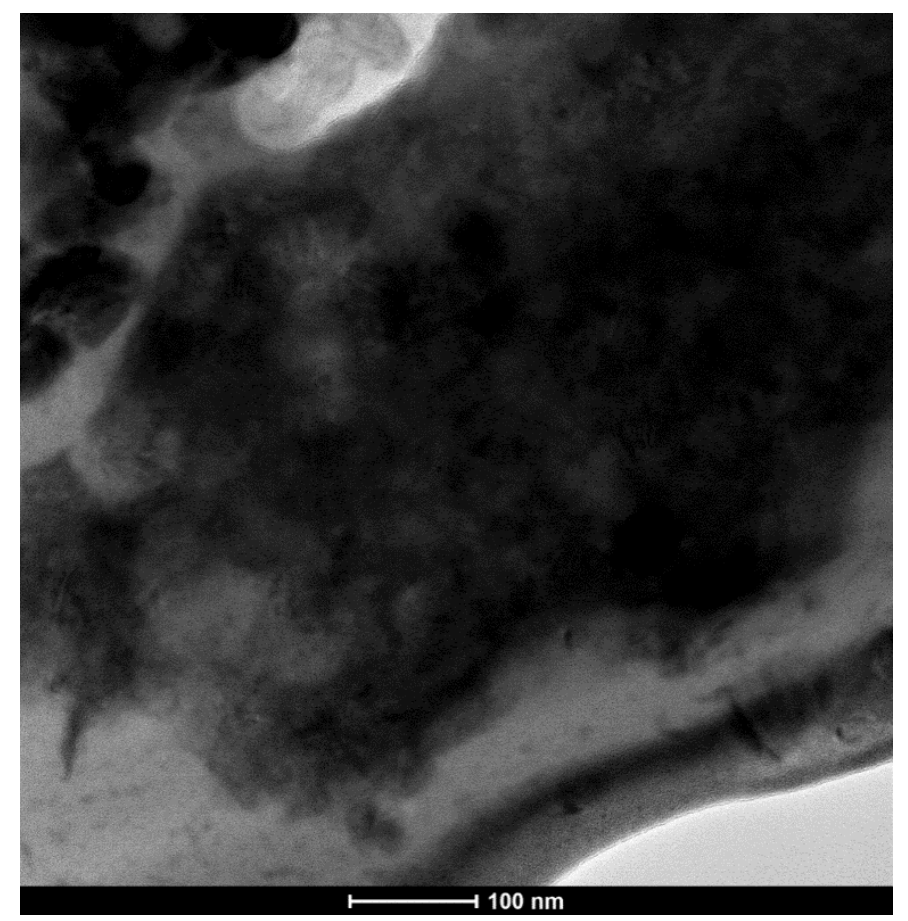

(a)

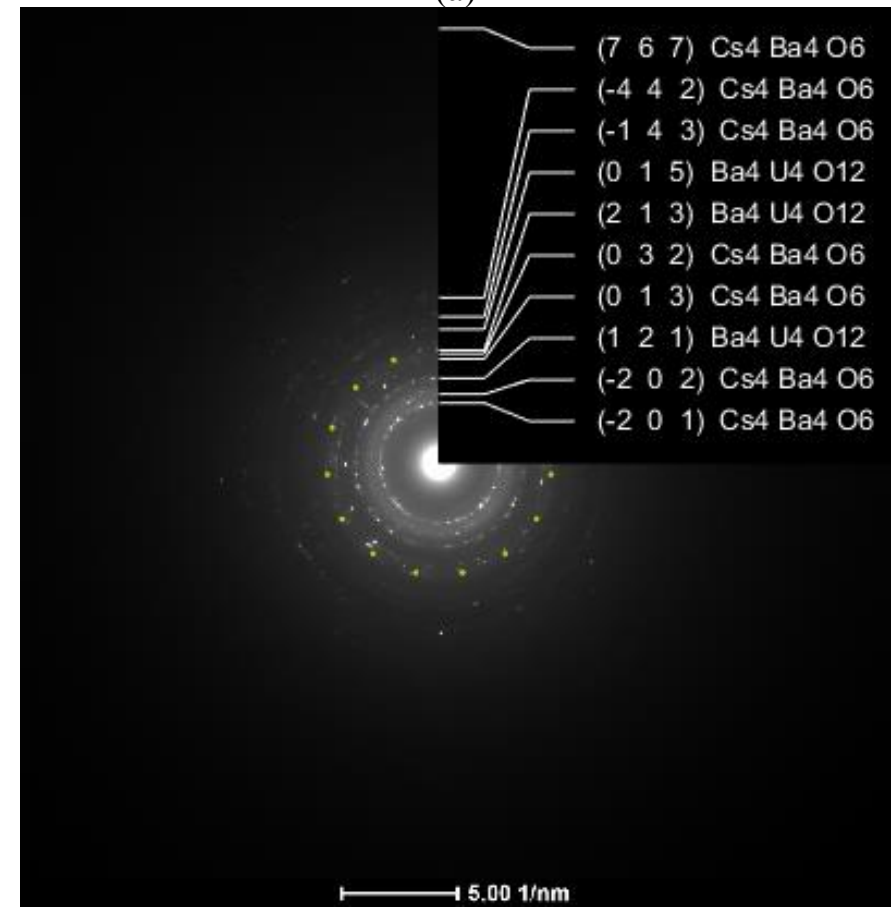

(b)

Figure 75. (a) Ba-rich region probed for crystallographic information. (b) Corresponding ring pattern identified with a $\mathrm{Cs}_{2} \mathrm{Ba}_{2} \mathrm{O}_{3}$-type of structure. 
Lamella B was extracted to evaluate the interaction of the cladding components with the fission products. The interface was extremely fragile, and most of the lamella was lost during thinning. Two sides could be savaged, the part of the lamella closer to lamella A and the cladding side, which are displayed in Figure 76a and b, respectively. An enlarged image of the side toward the fuel is shown in Figure 77a. The oxygen map in Figure 77b shows that both an oxide phase and metallic phases are present, consistent with the scattered O signal already collected with EPMA in the area. The composition was heterogeneous across the area, but the main components are Cs and Fe outside the metallic phases, which are composed of $\mathrm{Fe}$ and $\mathrm{Pd}$ instead. In this case, $\mathrm{Fe}$ is the cladding element that has reacted with $\mathrm{Cs}$ and Pd. In the other part of the lamella, Fe has reacted with Pd and layered zones are present (see Figure 78b). A small section containing mainly $\mathrm{Cs}$ and $\mathrm{O}$, is deposited next to the most Pd-enriched layer. 


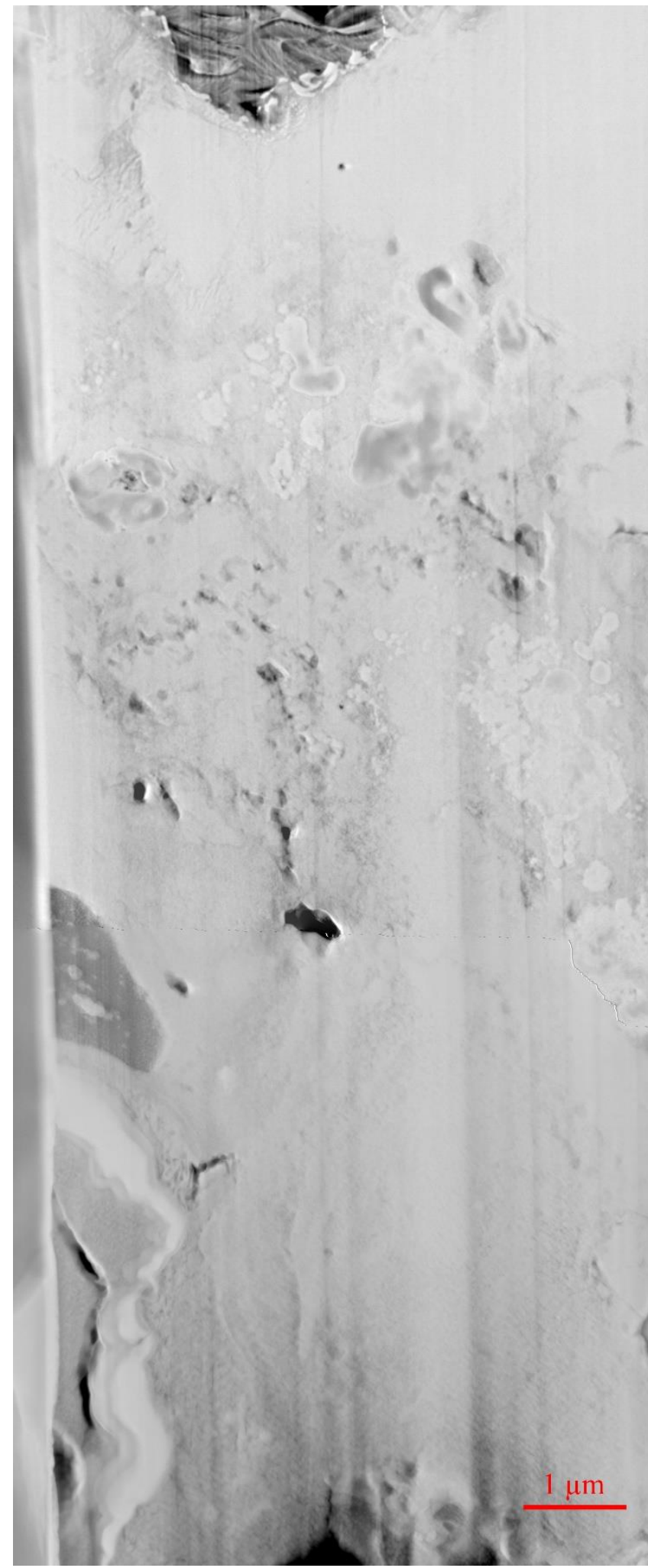

(a)

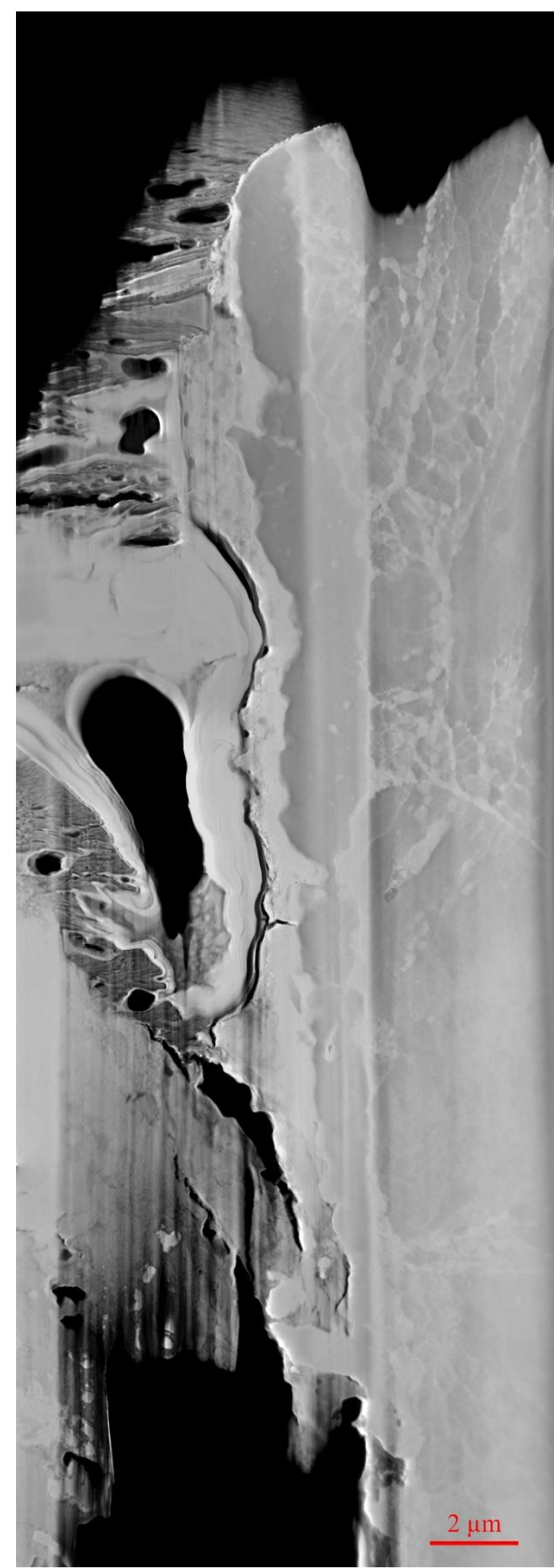

(b)

Figure 76. Overview of lamella B. (a) Side close to lamella A. (b) side capturing the interface with the cladding. 


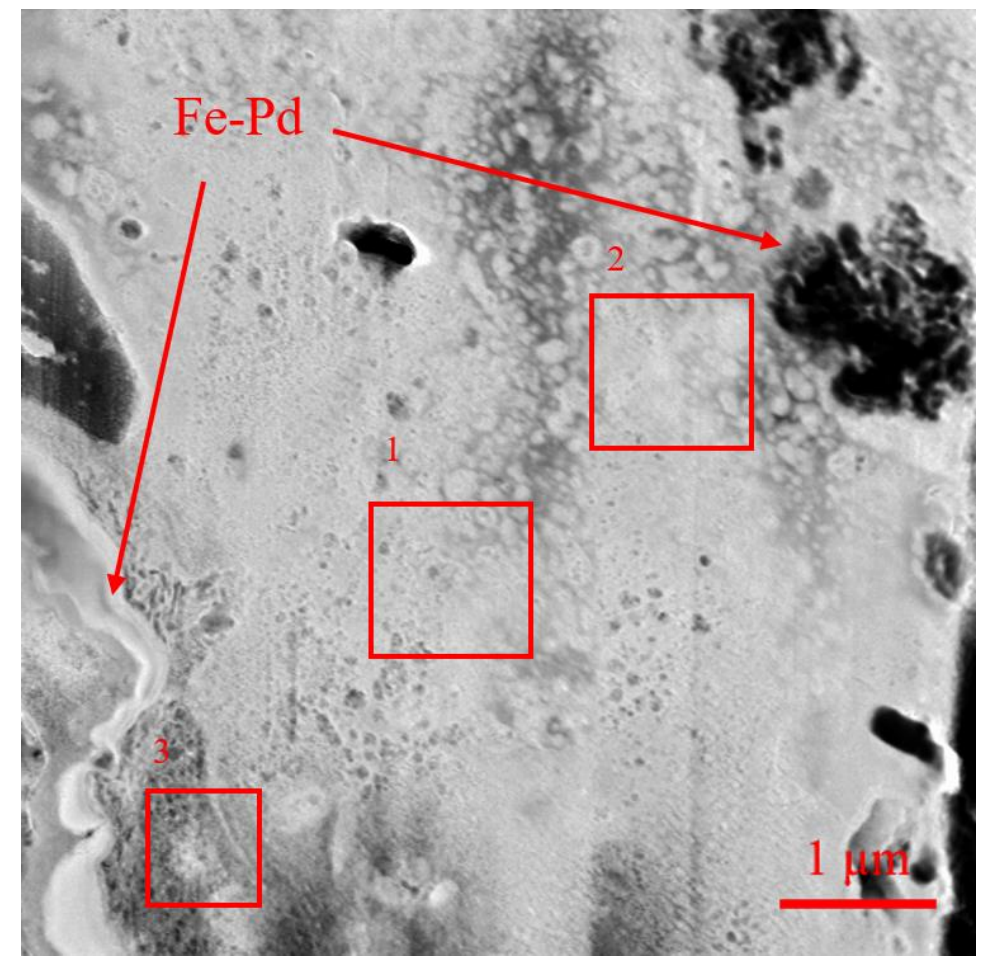

(a)

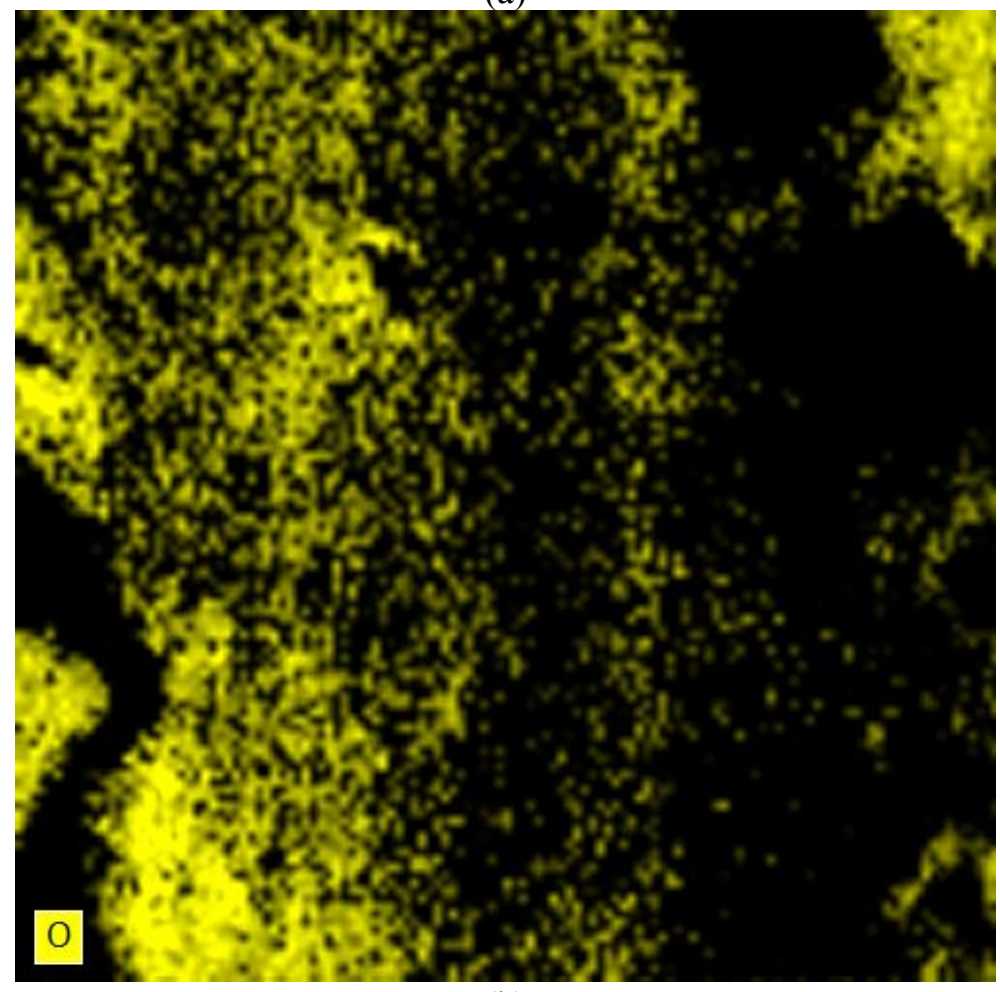

(b)

Figure 77. (a) HAADF image of the chemical mapping of the side of the lamella closes to the previous. (b) O qualitative map. 
Table 9. Composition of the three areas indicated in Figure 77a.

\begin{tabular}{lccc} 
& Area 1 & Area 2 & Area 3 \\
\hline $\mathrm{O}$ & 9.16 & 0.00 & 50.53 \\
$\mathrm{Fe}$ & 17.99 & 20.09 & 14.77 \\
$\mathrm{Pd}$ & 4.29 & 3.57 & 2.69 \\
$\mathrm{Te}$ & 3.78 & 5.83 & 1.28 \\
$\mathrm{I}$ & 3.25 & 2.66 & 3.01 \\
$\mathrm{Cs}$ & 54.28 & 60.12 & 20.33 \\
$\mathrm{Ba}$ & 1.68 & 0.00 & 0.31 \\
$\mathrm{U}$ & 5.57 & 7.73 & 0.06 \\
Other minor & & & \\
components & n.a & n.a & 7.03 \\
\hline
\end{tabular}



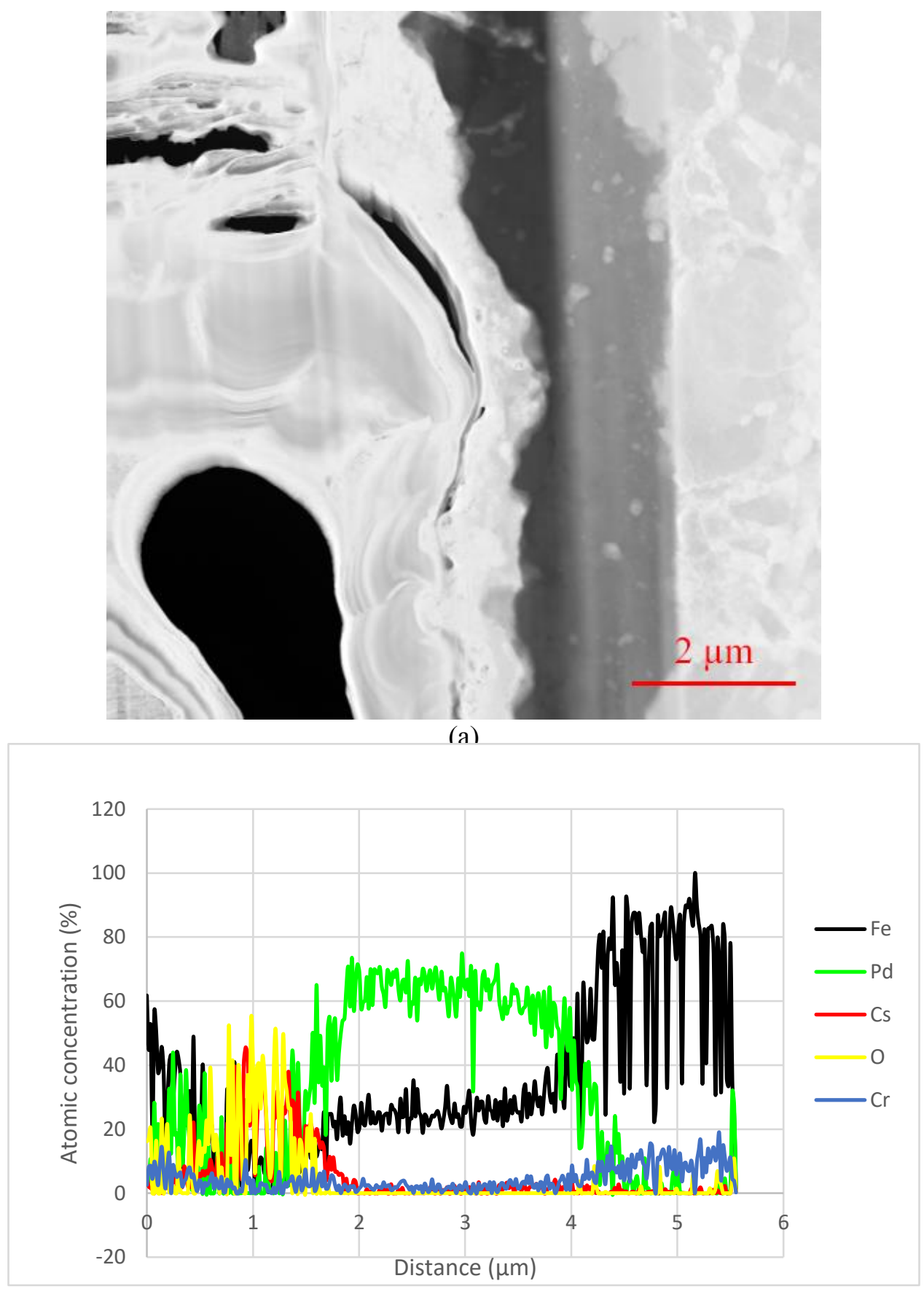

(b)

Figure 78. (a) Area closest to the cladding. (b) line scan of the major components.

\subsubsection{Actinides and fission products radial redistribution measured by EPMA}

In addition to the analyses of the FCCI, EPMA was used to determine quantitative radial distribution of actinide species and major fission products to evaluate their radial distribution due to vapor and solid thermo-migration. Figure 79 shows the radial distribution of $\mathrm{U}$ and $\mathrm{Pu}$, as well as the $\mathrm{Am}$ and $\mathrm{Np}$ produced by capture. The enrichment of Pu towards the central void is consistent with the Soret effect predicted for SFR MOX fuels and readily observed in literature [33-35]. The signal becomes scattered 
toward the fuel periphery. Some variability could be due to the imperfect status of the sample surface, which was very cracked particularly towards the periphery. However, both the radial quantitative profile and some of the atomic maps shown previously (see for example Figure 67b and Figure 68c) highlight Pu enrichment toward the periphery. In fast reactor, the mean free path of neutrons is large compared to the pin diameter, and it is difficult to justify the enrichment as a consequence of enhanced neutron capture by U-238 as it occurs in LWR fuel. More likely, the local enrichment could be due to fabrication inhomogeneities between the blended $\mathrm{UO}_{2}-\mathrm{PUO}_{2}$ starting powders, which have been reported in fabrication reports [36]. Figure 80a shows the volatile Cs, which has accumulated in the periphery as already shown by the SEM and TEM analyses in previous paragraphs. Figure $80 \mathrm{~b}$ reports $\mathrm{Zr}$ and $\mathrm{Ba}$, while Figure $80 \mathrm{c}$ shows the profile of $\mathrm{Nd}$, which was similar to other two lanthanides, $\mathrm{La}$ and $\mathrm{Pr}$. $\mathrm{Ba}$ accumulation toward the equiaxed region could be attributed to thermal migration in accordance with the results reported in [37]. However, the interpretation of Ba profile in our case is complicated by the decay of Cs-137 occurred in the $>20$ years of storage of this fuel. The behavior of $\mathrm{Zr}$ is also interesting, showing clear accumulation at the interface between columnar and equiaxed region. A similar behavior was observed by Sato et al. [38] in higher burnup MOX fuel, but the causes are not understood at the moment. $\mathrm{Zr}$ stable istotopes have volatile precursors that could influence the final location of $\mathrm{Zr}$ along the radius. $\mathrm{Zr}$ and $\mathrm{Ba}$ influence their respective solubility in the MOX matrix [37], and their precipitation will depend on the relative concentration of each at the specific radial location. More studies targeted to these two fission products would be needed to fully unravel the causes that determine this radial profile. Finally, the $\mathrm{Nd}$ profile is depicted in Figure 80c, as representative of immobile lanthanides used as burnup monitor. The Nd increase towards the central void and at the periphery is consistent with Pu enrichment, and caused local fluctuations in burnup.

The radial burnup profile derived from Nd concentration and assuming the fission split determined via wet chemistry methods (see Ref. [1]) is shown in Figure 81. The EPMA-based burnup calculations would predict a slightly lower pellet average burnup (5.65\% FIMA vs 6.32\% FIMA), but the burnup quantitative determination via EPMA is more sensitive to errors in the determination of the fission split; hence, it would be more advisable to use the EPMA data only for a radial qualitative estimation. The trend highlights the important consequences in $\mathrm{Pu}$ redistribution in terms of fission rate along the pellet radius. 


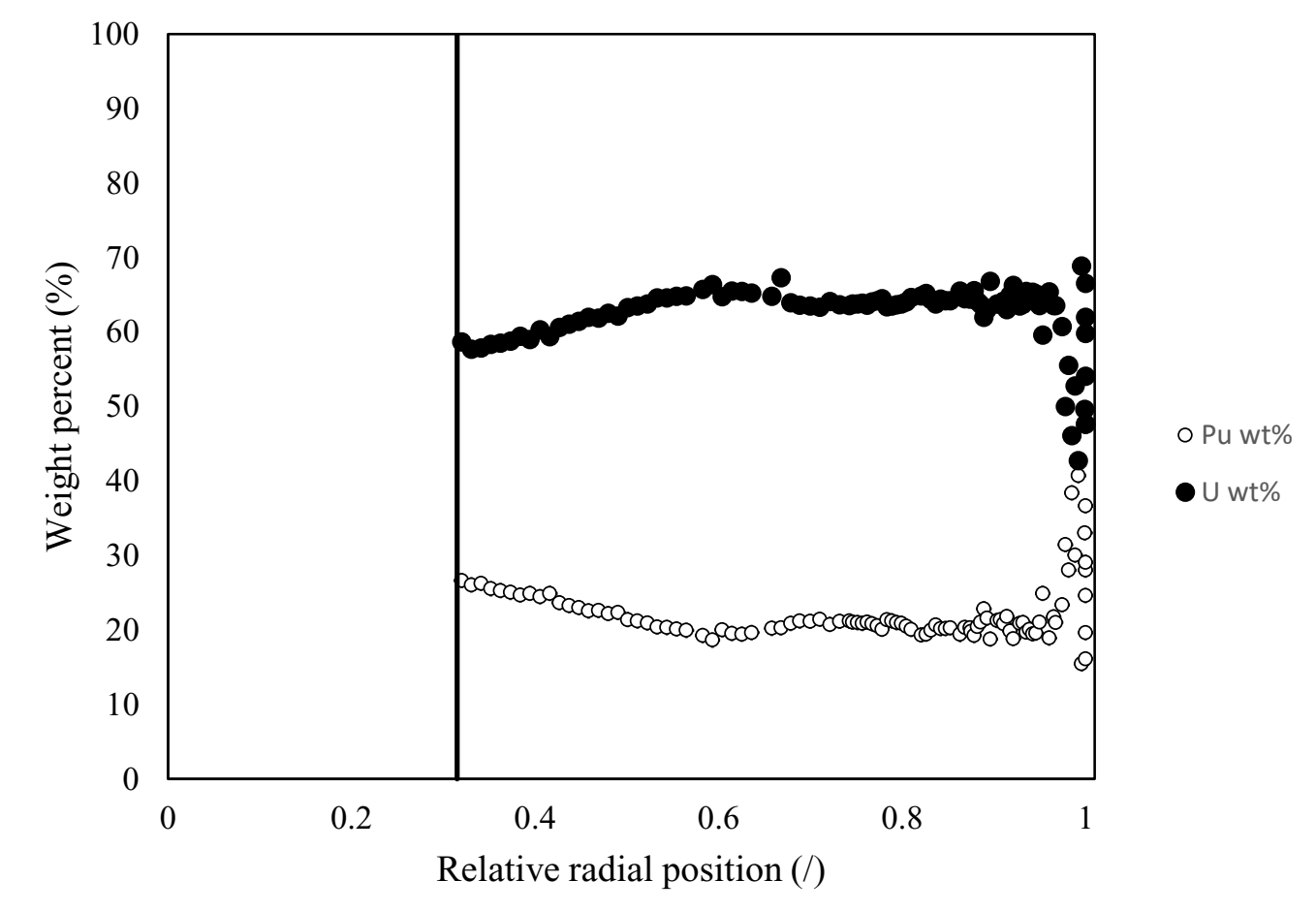

(a)

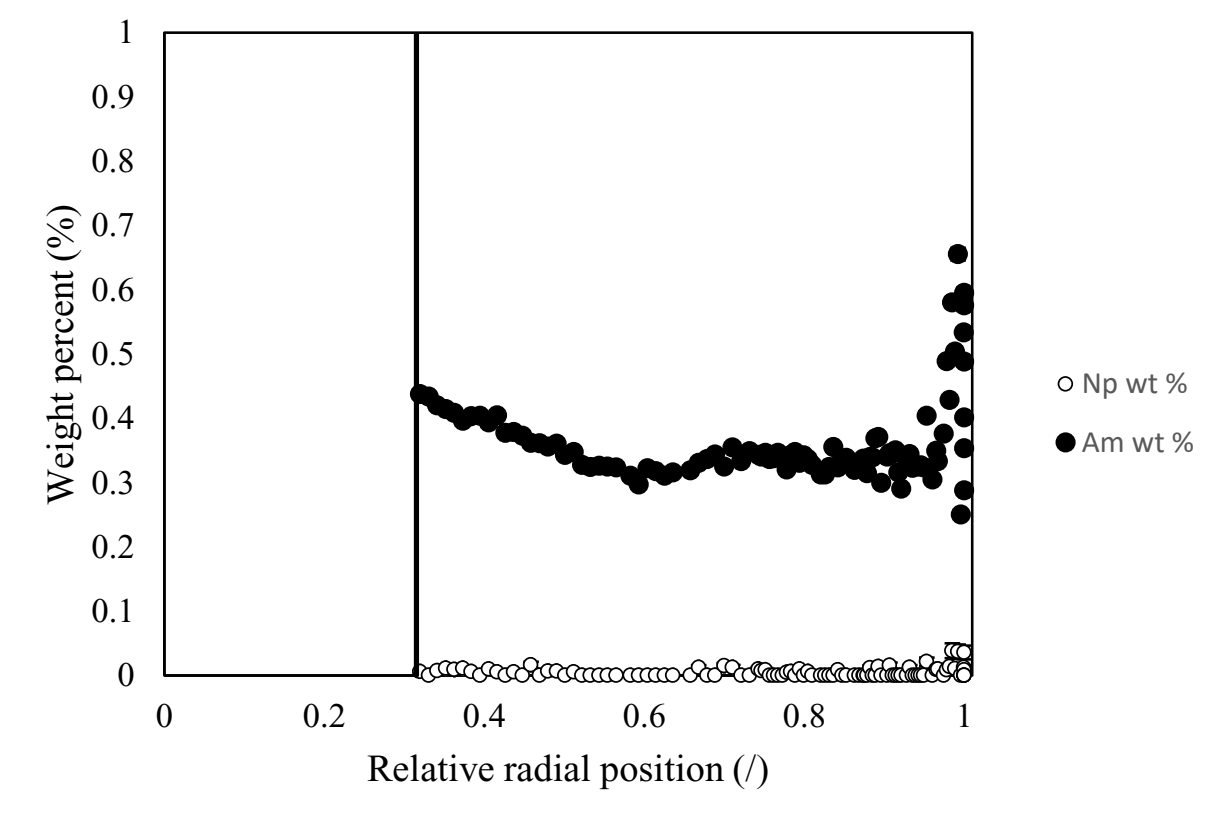

(b)

Figure 79. (a) Radial quantitative profile of (a) $\mathrm{U}$ and $\mathrm{Pu}$, showing actinide thermo-migration. (b) Am and $\mathrm{Np}$. 


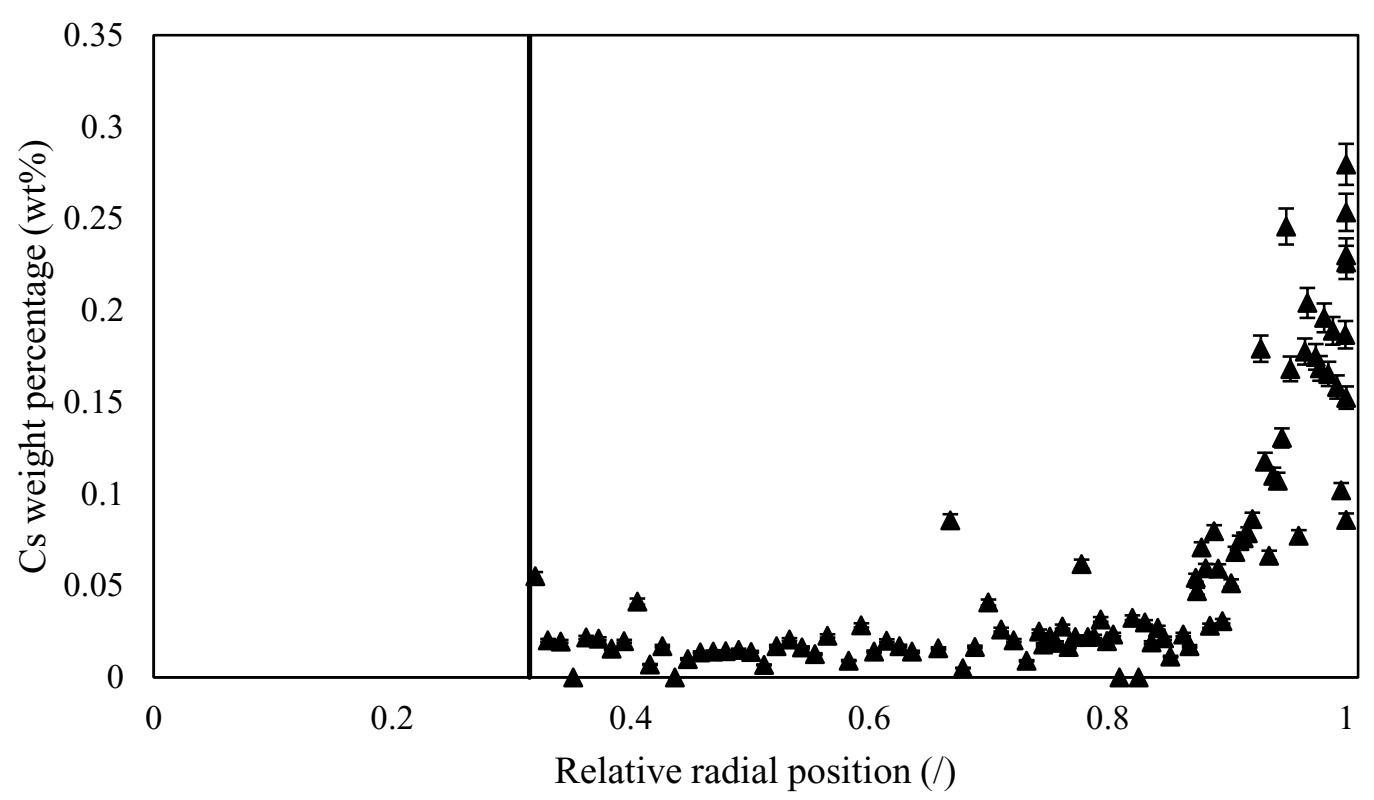

(a)

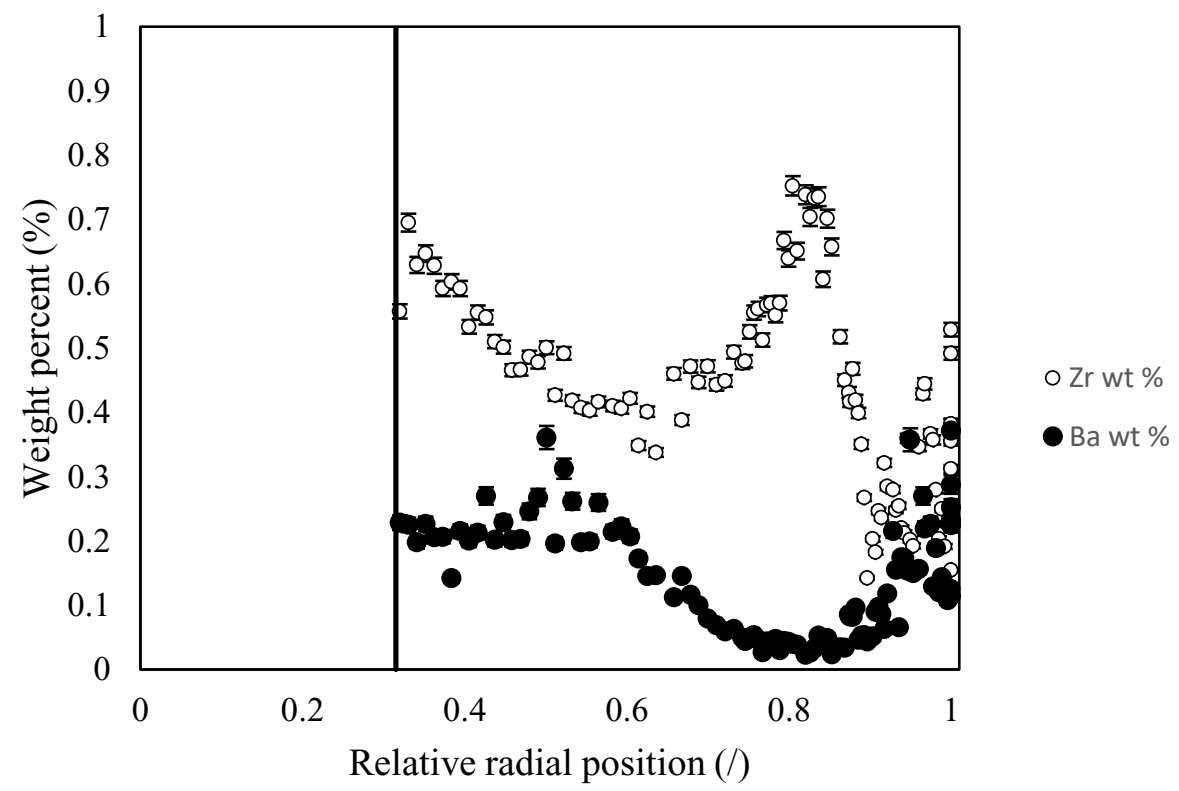

(b) 


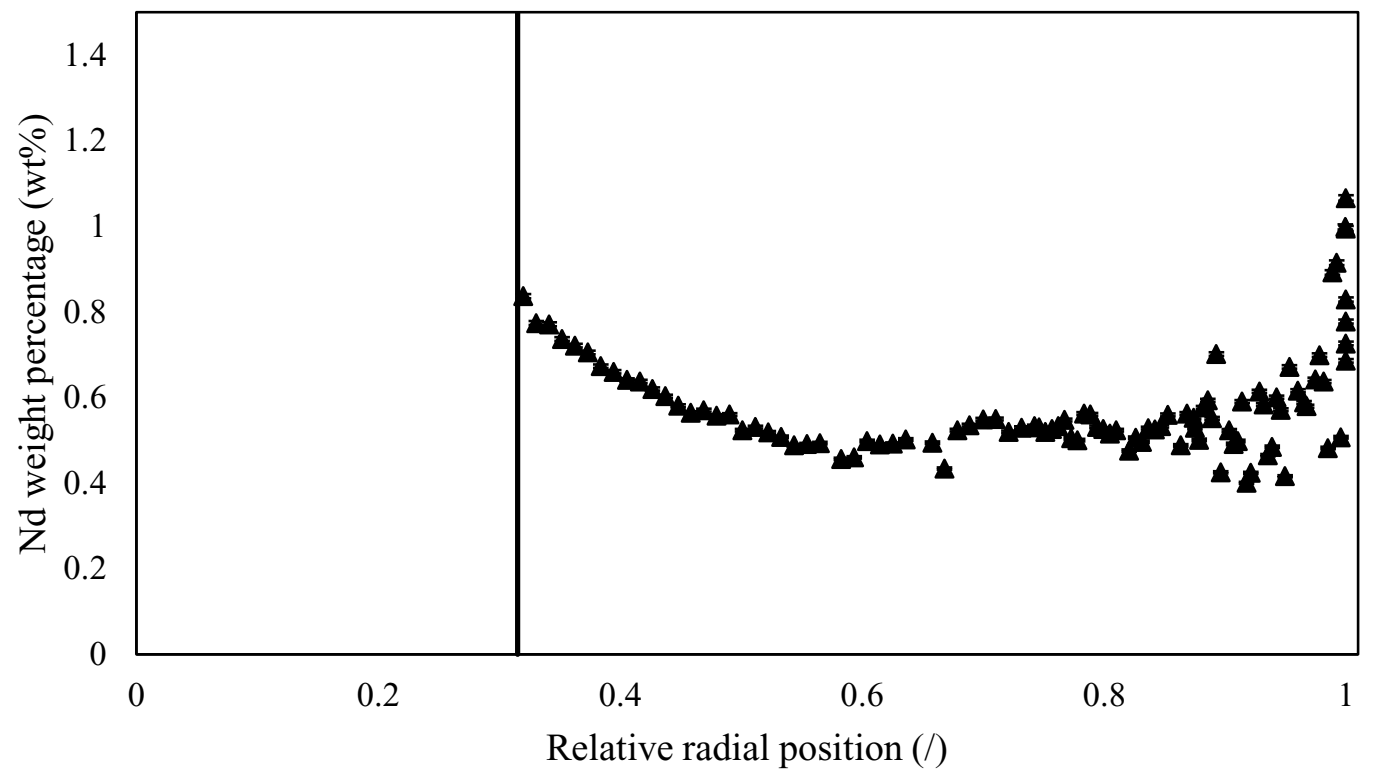

(c)

Figure 80. Radial quantitative profile of (a) $\mathrm{Cs}$, (b) $\mathrm{Zr}$ and $\mathrm{Ba}$, and (c) $\mathrm{Nd}$.

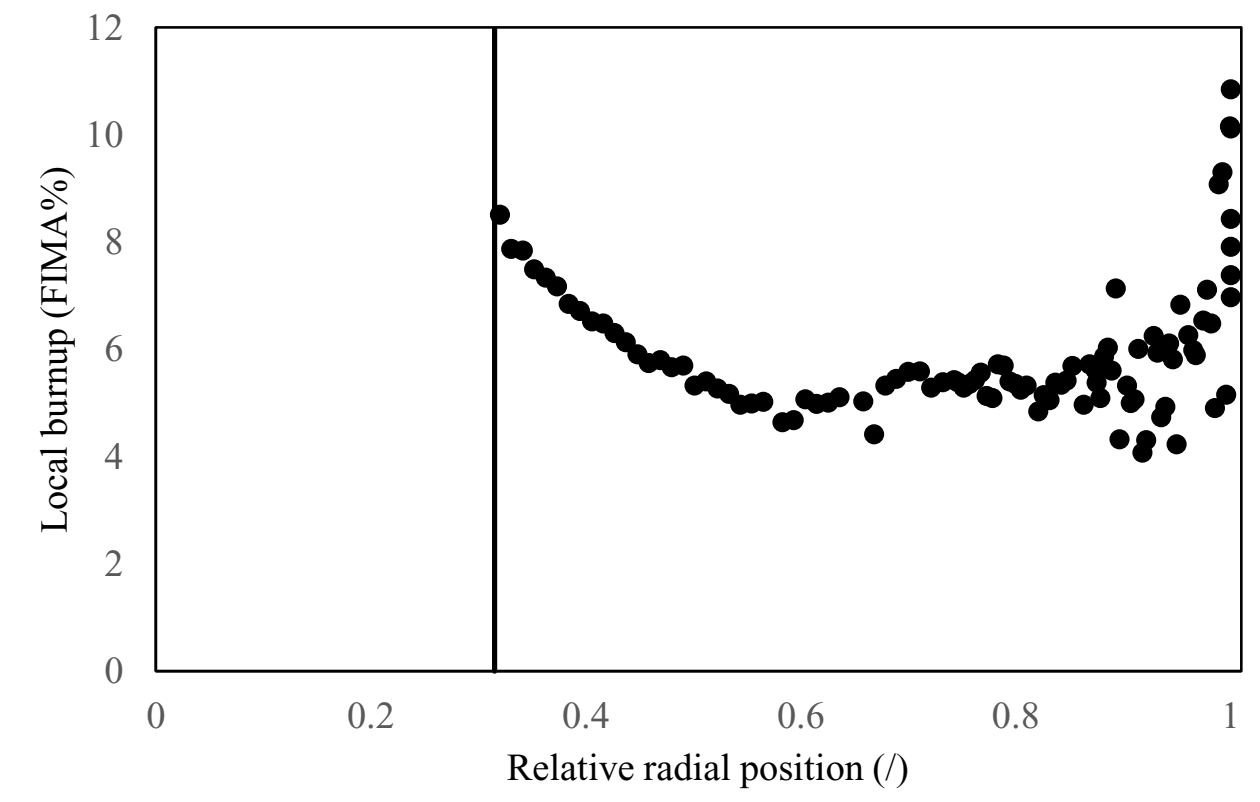

Figure 81. Radial burnup profile derived from $\mathrm{Nd}$ measurements. 


\subsubsection{Sample 4}

\subsubsection{Fuel microstructure and fission products}

The overview of the last sample is shown in Figure 82. The observations made on this sample largely repeat what was reported for the previous samples. As can be noticed from the optical overview, a large portion of the structure pulled out, leaving areas of "fresh fracture" open to investigation. As seen for the other sample from K06, the grain surface of the grains close to the central hole is decorated with large metallic precipitate inclusions, often associated with porosity (Figure 83). Large tunnels have formed along the grain boundaries, consistently with the large fission gas release measured during the baseline PIE campaign. The secondary phases encountered were mainly five metal precipitates. Also in this case, the $\mathrm{Mo} / \mathrm{Ru}$ ratio remined close to one, confirming that negligible portion of Mo have oxidized. The observation of grey phases was occasional. A large agglomerate, whose approximate composition is given in Table 10, was seen at the interface of the columnar and equiaxed region (see Figure 84), in agreement with the trend reported by Kurosaki et al. [39] for a sample with higher burnup, showing accumulation of $\mathrm{Ba}$ and $\mathrm{Zr}$ between the two regions.

At the outermost periphery of the fuel pellet, localized grain subdivision was present as for sample 1 and 2. Representative examples are shown in Figure 85 and Figure 86. The subdivision does not extend more than $100 \mu \mathrm{m}$ inside the fuel, and, in some cases, it was observed where the FCCI thickness was the larger, with considerable portion of the fuel which remained attached to the cladding (Figure 87).

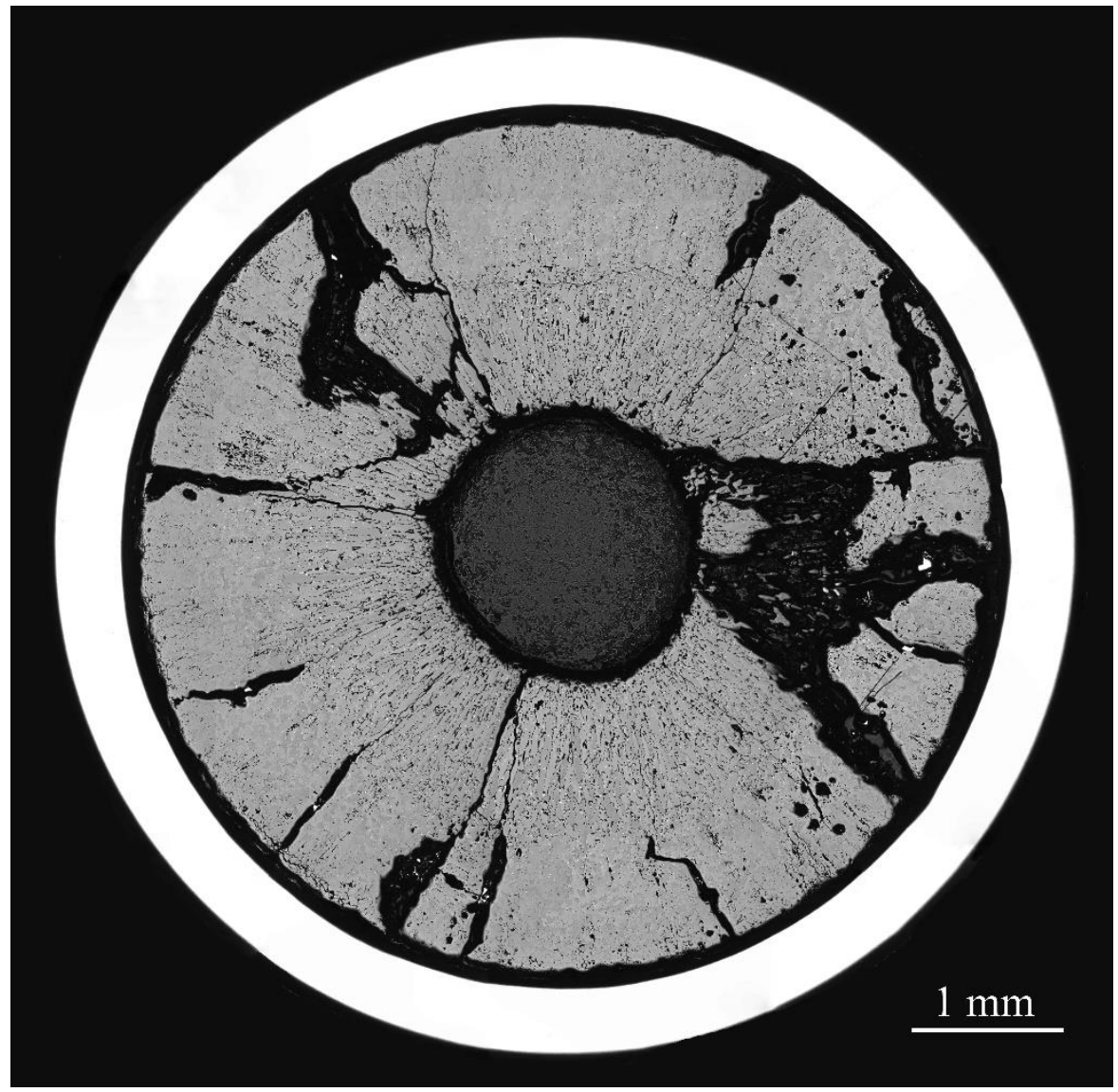

Figure 82. Overview of sample 4. 


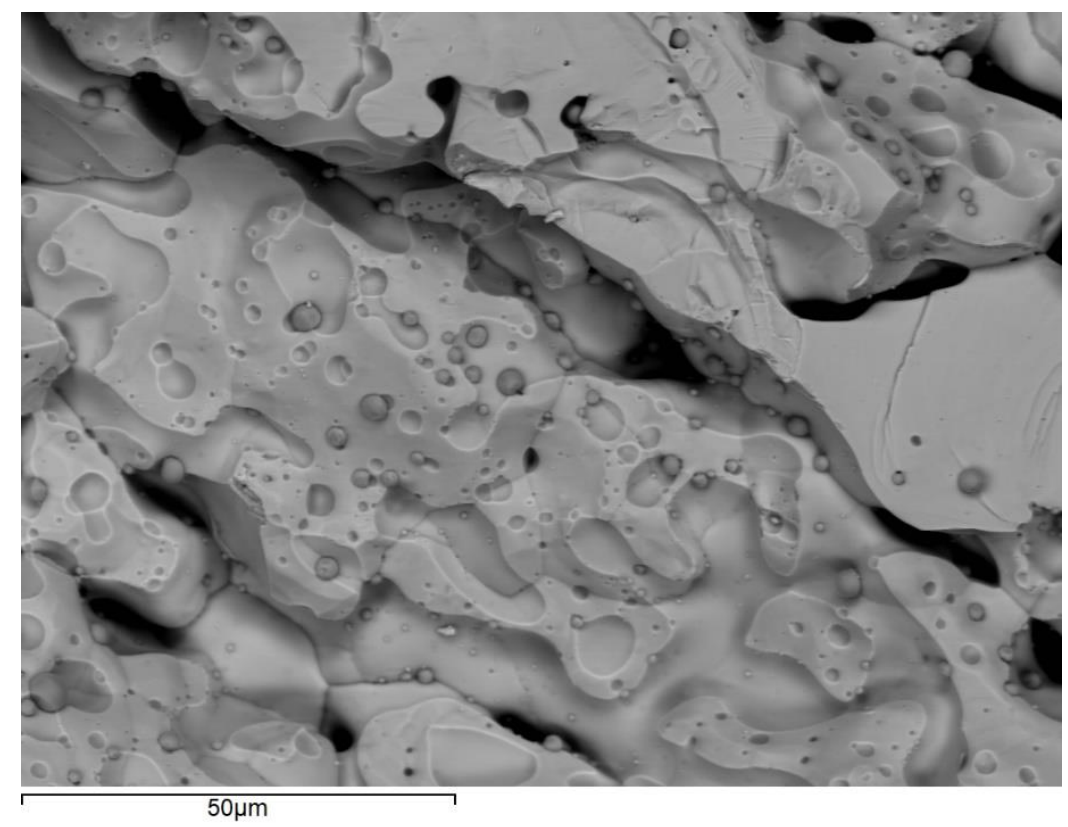

Figure 83. Fresh fracture image of the columnar grains close to the central hole.

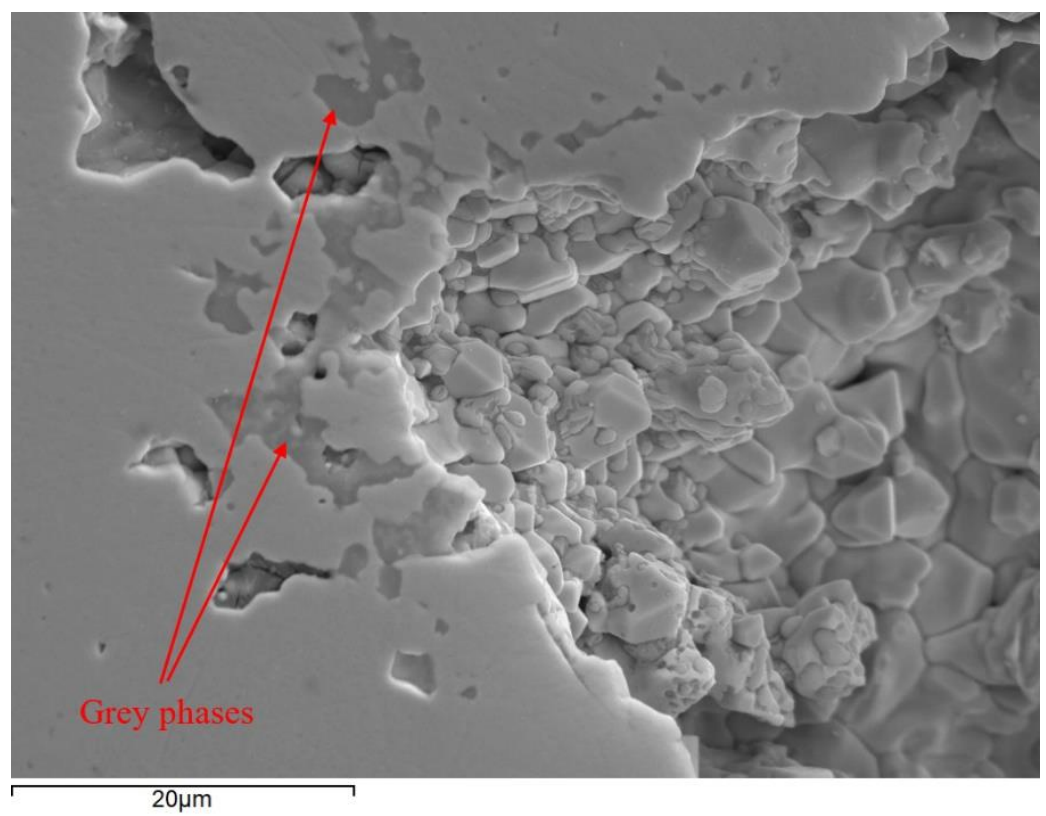

Figure 84. Grey phases observed between the columnar and equiaxed grain region. 
Table 10. Semi-quantitative composition of the grey phases measured by EDS.

\begin{tabular}{|c|c|c|}
\hline Element & Weight $\%$ & Error (wt \%) \\
\hline $\mathrm{O}$ & 29.24 & 0.21 \\
\hline $\mathrm{Zr}$ & 18.05 & 0.13 \\
\hline $\mathrm{Ba}$ & 34.29 & 0.18 \\
\hline $\mathrm{Ce}$ (from Ba decay) & 0.12 & 0.14 \\
\hline $\mathrm{U}$ & 17.81 & 0.17 \\
\hline $\mathrm{Pu}$ & 0.5 & 0.18 \\
\hline
\end{tabular}

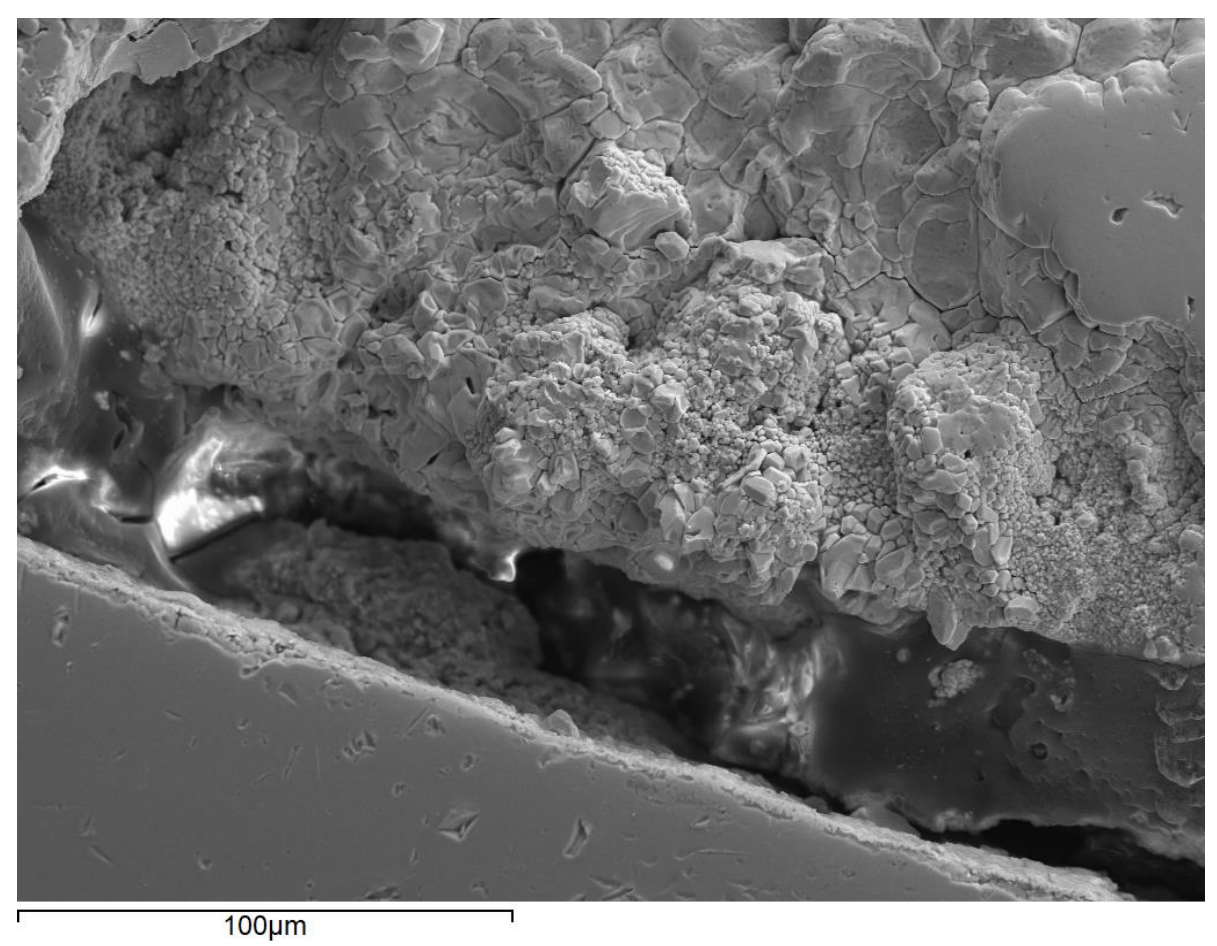

Figure 85 . grain morphology at the fuel periphery. 


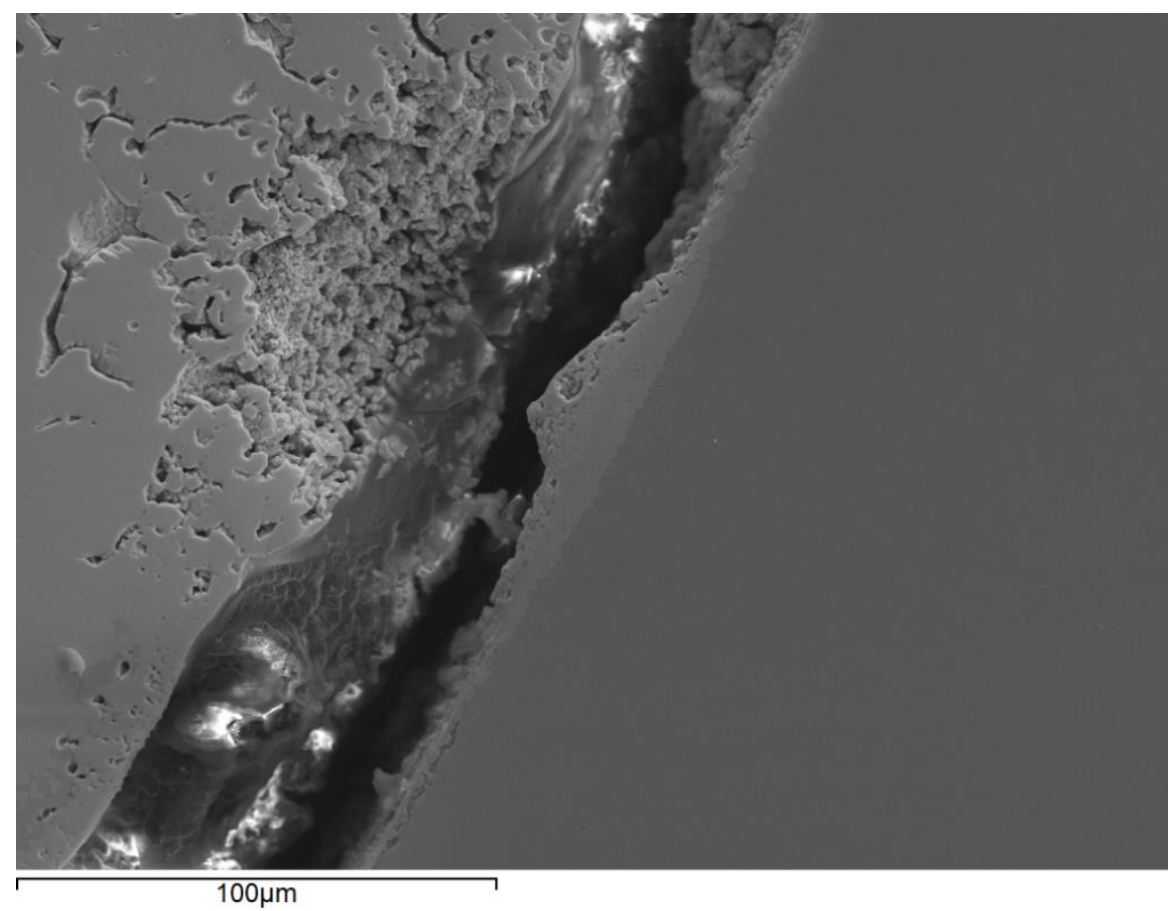

Figure 86. Other location where grain subdivision was observed. On the right side of the image, it can be seen that part of the fuel has remained attached to the FCCI.

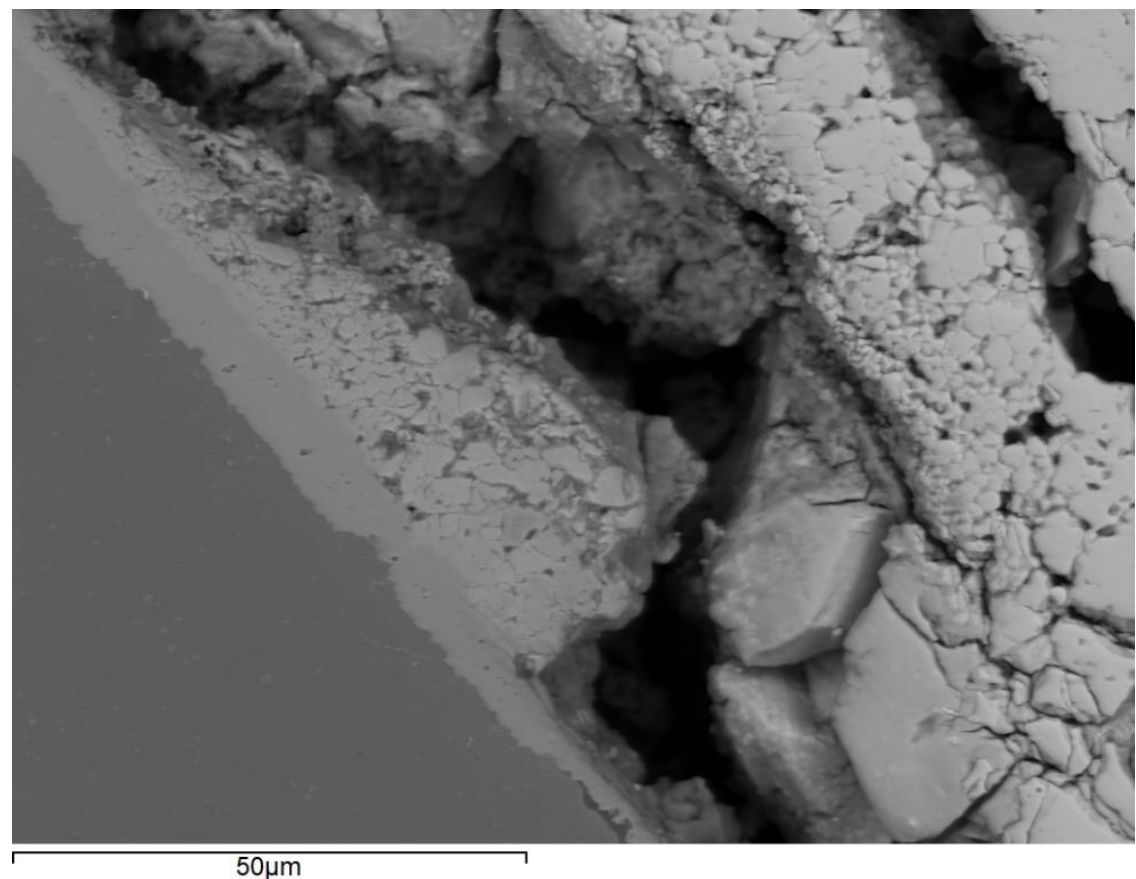

Figure 87. Region where a thick part of the fuel remained attached to the cladding. 


\subsubsection{Fuel-cladding chemical interaction}

Regarding the FCCI observed in this sample, the observations were similar to the other samples. Typical appearance of the FCCI has been shown in the previous images (Figure 85 to Figure 87). The front of the interaction toward the cladding was characterized by the Pd liner, with a more porous region extending toward the fuel, which contained in addition to the fuel components, mainly the volatile Cs, Te and I.

The primary purpose of these analyses was the determination of the FCCI between MOX and HT-9 cladding, which has not been extensively evaluated to date. Hence, the discussion is primarily focused on this phenomenon. For instance, determination of the five metals precipitate composition in the various samples and at different radial locations was mainly focused on the assessment of $\mathrm{Mo} / \mathrm{Ru}$ content. The interest in determining whether Mo is still contained in the metallic precipitates comes from the need to determine the fuel oxygen potential, which is a primary parameter in driving the cladding corrosion. The various examinations of the metallic inclusions presented in the previous section prove that, for all the samples, Mo is still in the metallic phase. Hence, the oxygen potential in the fuel must have always been below the $\mathrm{Mo} / \mathrm{MoO}_{2}$ value.

The nature of the fuel-cladding interaction zone between the two fuels presents similarities at the "meso-scale" level, but notable differences at the microscopic level as highlighted by the TEM analyses. The SEM investigations showed how the cladding interaction areas contained mainly $\mathrm{Cs}$, Te, and a local presence of I. Pd was also largely present, mainly in form of a liner attached to the cladding. The cladding components and the local distribution of the fission products at microscopic level, however, presented several differences both between the samples belonging to the same rod taken at different axial locations (meaning, with different cladding temperatures) and between samples at the same axial location but from the two rods.

When comparing the results from sample 1 and 2, or 3 and 4 the amount of diffusion of cladding component into the fuel clearly increases in the sample in the upper half of the fuel stack despite the lower burnup, confirming that the cladding temperature is a stronger parameter than burnup in determining the extent of interaction for FCCI. The cladding component that diffused most was $\mathrm{Cr}$, creating zones enriched with up to 80 at\% next to areas containing $\mathrm{Cs}$, Te and, in some cases I. As discussed previously, the fact that $\mathrm{Cr}$ is not oxidized, in addition to the shape and morphology of the fission product deposits, suggests a diffusion aided by liquid fission products, mainly Cs. FCCI-type of corrosion is not excluded though, as a case of $\mathrm{Cr}$ oxide was seen (Figure $37 \mathrm{~b}$ and d). In addition, at the interface with the nanocrystalline ponds containing $\mathrm{Cs}$, co-presence of $\mathrm{Cr}$ and $\mathrm{Cs}$ might suggest initial oxidation of the $\mathrm{Cr}$ to form $\mathrm{Cs}_{2} \mathrm{CrO}_{4}$.

In the sample from the other rod what rod?, Fe has diffused towards the fuel instead. Fe transport outside the cladding has been associated with I transport, thanks to the increase I partial pressure from radiation-induced CsI decomposition [40,41]. The present TEM data does not show considerable presence of I, rather Cs, and further closer to the fuel Te are again the major fission product detected. Certainly the lower yield of I with respect to Cs and Te plays a role in the probability of detection, but it has to be noted that I is particularly volatile and we cannot exclude that part of it has been lost during sample preparation. An alternative transport mechanism of $\mathrm{Fe}$ has been proposed by Fitts [8], which includes transport of iron oxides through a molten layer of $\mathrm{Cs}_{2} \mathrm{O}$. Such mechanism would be more compatible with the chemical composition of the interaction layer measured in the sample. At present, no final explanation of the different nature of the FCCI of the sample from L08 is available. Some hypothesis could be made. The higher burnup of this sample, highest among the ones analyzed, could be sufficient to determine an oxygen potential compatible with Fe oxidation that would result in Fe transport by the aforementioned mechanism. However, Cr oxides would be expected to have formed first, which was not observed, making this explanation doubtful. 


\section{CONCLUSIONS}

The microstructural analyses conducted brought two major observations and conclusions, one related to the microstructural modifications of the fuel and the second related to the nature of the fuel to cladding interaction. Regarding the first one, the periphery of the fuel has experienced grain subdivision, which has been observed either on pre-existing open surfaces or where considerable accumulation of fission products, mainly Cs, was present. The restructured grains are similar to the recrystallized grains of the HBS in LWR, but here the micrometric pores are not present.

With respect to the FCCI, the areas of interactions were not homogeneous, rather concentrated in islands with different thickness and composition. In all the samples, the fission products $\mathrm{Pd}, \mathrm{Cs}$ and $\mathrm{Te}$ accumulated in the fuel-to-cladding gap region. Pd formed a thin liner layer of various thickness alloyed with Fe. The volatiles have been found mostly in nanocrystalline pockets, probably as a result of solidification upon reactor shut down. In the samples from $\mathrm{K} 06, \mathrm{Cr}$, still in metallic form, was the cladding component that has migrated into the fuel, enriching the outermost periphery of the pellet in contact with the HT-9 cladding. The morphology and composition of the deposits suggest a FPLME-induced corrosion mechanism. In the sample from pin L08, both $\mathrm{Fe}$ and $\mathrm{Cr}$ have started diffusion towards the fuel. Coexistence of metallic and oxidized regions were observed, but the results are not conclusive to determine the underlying corrosion mechanism. 


\section{REFERENCES}

[1] F. Cappia, K. Tanaka, M. Kato, K. McClellan, J. Harp, "Post-irradiation examinations of annular mixed oxide fuels with average burnup 4 and 5\% FIMA", J. Nucl. Mater. 533 (2020) p. 152076. https://doi.org/10.1016/j.jnucmat.2020.152076.

[2] R.J. Parrish, K.E. Wright, A.J. Winston, C. McKinney, J.M. Harp, A. Aitkaliyeva, "Characterization of solid fission products in 13.7\% FIMA MOX fuel using electron microscopy techniques", J. Nucl. Mater. (2019). https://doi.org/10.1016/j.jnucmat.2019.06.042.

[3] R.J. Parrish, X. Liu, A. Winston, J.M. Harp, A. Aitkaliyeva, "Radial microstructural evolution in low burnup fast reactor MOX fuel", J. Nucl. Mater. 523 (2019) p. 182-188. https://doi.org/10.1016/j.jnucmat.2019.06.009.

[4] M. Klinger, "More features, more tools, more CrysTBox", J. Appl. Crystallogr. 50 (2017). $10.1107 /$ S1600576717006793.

[5] H. Kleykamp, "Die radiale sauerstoff-verteilung im brennstoff bestrahlter mischoxidbrennstäbe unterschiedlicher ausgangsstöchiometrie", J. Nucl. Mater. 66 (1977) p. 292-300. https://doi.org/10.1016/0022-3115(77)90118-0.

[6] M. Tourasse, M. Boidron, B. Pasquet, "Fission product behaviour in Phenix fuel pins at high burnup", J. Nucl. Mater. (1992). 10.1016/0022-3115(92)90453-R.

[7] I. Sato, H. Furuya, T. Arima, K. Idemitsu, K. Yamamoto, "Behavior of metallic fission products in uranium-plutonium mixed oxide fuel", J. Nucl. Mater. 273 (1999) p. 239-247. https://doi.org/10.1016/S0022-3115(99)00071-9.

[8] R.B. Fitts, E.L. Long, J.M. Leitnaker, "Observations of fuel-cladding chemical interactions as applied to GCBR fuel rods", in: Fast Reactor Fuel Element Technology, pp. 431-458.

[9] M.H. Rand, P.E. Potter, "Thermodynamics and phase diagrams of Mo-Pd-Ru and related systems", Phys. B+C. 103 (1981) p. 21-30. https://doi.org/10.1016/0378-4363(81)90999-2.

[10] H. Okamoto, "Mo-Pd (molybdenum-palladium)", J. Phase Equilibria. 13 (1992) p. 332-334. 10.1007/BF02667570.

[11] S.C. Middleburgh, D.M. King, G.R. Lumpkin, "Atomic scale modelling of hexagonal structured metallic fission product alloys", R. Soc. Open Sci. 2 (2020) p. 140292. 10.1098/rsos. 140292.

[12] M.G. Adamson, E.A. Aitken, T.B. Lindemer, "Chemical thermodynamics of Cs and Te fission product interactions in irradiated LMFBR mixed-oxide fuel pins", J. Nucl. Mater. 130 (1985) p. 375-392. https://doi.org/10.1016/0022-3115(85)90325-3.

[13] M.G. Adamson, E.A. Aitken, "On the Cs,Te fission product-induced attack and embrittlement of stainless steel cladding in oxide fuel pins", J. Nucl. Mater. 132 (1985) p. 160-166. https://doi.org/10.1016/0022-3115(85)90410-6.

[14] J.E. Antill, J.B. Warburton, "Influence of tellurium on caesium-enhanced corrosion of stainless steel", J. Nucl. Mater. 71 (1977) p. 134-139. https://doi.org/10.1016/0022-3115(77)90197-0.

[15] IAEA, "Technical Committee Meeting on Fuel and Cladding Interaction - Summary Report", (1977).

[16] J.-F. Vigier, P.M. Martin, L. Martel, D. Prieur, A.C. Scheinost, J. Somers, "Structural Investigation of (U0.7Pu0.3)O2-x Mixed Oxides", Inorg. Chem. 54 (2015) p. 5358-5365. 10.1021/acs.inorgchem.5b00392.

[17] I.L.F. Ray, H. Matzke, H.A. Thiele, M. Kinoshita, "An electron microscopy study of the RIM 
structure of a UO2 fuel with a high burnup of 7.9\% FIMA", J. Nucl. Mater. 245 (1997) p. 115123. https://doi.org/10.1016/S0022-3115(97)00015-9.

[18] V. V Rondinella, T. Wiss, "The high burn-up structure in nuclear fuel", Mater. Today. 13 (2010) p. 24-32. https://doi.org/10.1016/S1369-7021(10)70221-2.

[19] J. Spino, D. Papaioannou, I. Ray, "Rim formation and fission gas behaviour: some structure remarks", in: Fission Gas Behaviour in Light Water Reactor Fuels, Cadarache, France, pp. 247268.

[20] J. Spino, K. Vennix, M. Coquerelle, "Detailed characterisation of the rim microstructure in PWR fuels in the burn-up range 40-67 GWd/tM", J. Nucl. Mater. 231 (1996) p. 179-190. https://doi.org/10.1016/0022-3115(96)00374-1.

[21] J. Noirot, L. Desgranges, J. Lamontagne, "Detailed characterisations of high burn-up structures in oxide fuels", J. Nucl. Mater. 372 (2008) p. 318-339. https://doi.org/10.1016/j.jnucmat.2007.04.037.

[22] Y. Guerin, "Fuel Performance of Fast Spectrum Oxide Fuel", in: Comprehensive Nuclear Materials, R.J.M. Konings (Ed.), Elsevier, pp. 547-578. 10.1016/B978-0-08-056033-5.00043-4.

[23] K. Maeda, K. Tanaka, T. Asaga, H. Furuya, "Distributions of volatile fission products in or near the fuel-cladding gap of the FBR MOX fuel pins irradiated to high burn-up", J. Nucl. Mater. 344 (2005) p. 274-280. https://doi.org/10.1016/j.jnucmat.2005.04.054.

[24] J.P. Berton, D. Baron, M. Cocquerelle, "Chemical stability and physical properties of cesium uranates", in: Advances in Fuel Pellet Technology for Improved Performance at High Burnup, p. 363.

[25] R.G.J. Ball, W.G. Burns, J. Henshaw, M.A. Mignanelli, P.E. Potter, "The chemical constitution of the fuel-clad gap in oxide fuel pins for nuclear reactors", J. Nucl. Mater. 167 (1989) p. 191-204. https://doi.org/10.1016/0022-3115(89)90442-X.

[26] O. Götzmann, P. Hofmann, F. Thümmler, "Attack upon the cladding of oxide fuel pins by fuel and fission products", J. Nucl. Mater. 52 (1974) p. 33-50. https://doi.org/10.1016/00223115(74)90023-3.

[27] D.E. Rasmussen, W.R. Jentzen, R.B. McCord, "Alternative oxide fuel pellet fabrication for irradiation testing", in: Pacific Coast Meeting of American Ceramic Society, HEDL-SA--1604-FP, San Diego, United States, . https://www.osti.gov/servlets/purl/6396299.

[28] G. Bergman, D.P. Shoemaker, "The determination of the crystal structure of the $\sigma$ phase in the iron-chromium and iron-molybdenum systems", Acta Crystallogr. 7 (1954) p. 857-865. $10.1107 / \mathrm{S} 0365110 \times 54002605$.

[29] E.H. Lee, P.J. Maziasz, A.F. Rowcliffe, "The structure and composition of phases occurring in austenitic stainless steels in thermal and irradiation environments", in: Symposium on Irradiation Phase Stability, Pittsburgh, PA, .

[30] H. Kleykamp, R. Pejsa, "X-ray diffraction studies on irradiated nuclear fuels", J. Nucl. Mater. 124 (1984) p. 56-63. https://doi.org/10.1016/0022-3115(84)90009-6.

[31] A.E. Dwight, D.R. O’Boyle, "Phase studies on fission product alloys", J. Nucl. Mater. 136 (1985) p. 280-283. https://doi.org/10.1016/0022-3115(85)90015-7.

[32] J. Sangster, A.D. Pelton, "The Cs-Te (cesium-tellurium) system", J. Phase Equilibria. 14 (1993) p. 246-249. 10.1007/BF02667821.

[33] D.R. Olander, "Fuel chemistry", in: Fundamental Aspects of Nuclear Reactor Fuel Elements, pp. 
$145-171$.

[34] K. Tanaka, S. Miwa, S. Sekine, H. Yoshimochi, H. Obayashi, S. Koyama, "Restructuring and redistribution of actinides in Am-MOX fuel during the first 24h of irradiation", J. Nucl. Mater. 440 (2013) p. 480-488. https://doi.org/10.1016/j.jnucmat.2013.01.351.

[35] C.T. Walker, G. Nicolaou, "Transmutation of neptunium and americium in a fast neutron flux: EPMA results and KORIGEN predictions for the superfact fuels", J. Nucl. Mater. 218 (1995) p. 129-138. https://doi.org/10.1016/0022-3115(94)00649-0.

[36] D.E. Rasmussen, E.. Gerber, R.B. McCord, "Microstructure characterization of advanced oxide fuel", in: 79th Annual Meeting and Exposition of the American Ceramic Society, Chicago, Illinois,

[37] C. Sari, C.T. Walker, G. Schumacher, "Solubility and migration of fission product barium in oxide fuel", J. Nucl. Mater. 79 (1979) p. 255-259. https://doi.org/10.1016/0022-3115(79)90455-0.

[38] I. Sato, H. Furuya, T. Arima, K. Idemitsu, K. Yamamoto, "Behavior of fission products zirconium and barium in fastreactor fuel irradiated to high burnup", J. Nucl. Sci. Technol. 36 (1999) p. $775-$ 780 .

[39] K. Kurosaki, K. Tanaka, O. Masahiko, Y. Ohishi, H. Muta, M. Uno, S. Yamanaka, "Chemical state of fission products and actinides in irradiated Oxide fuels analyzed by thermodynamic calculation and post-irradaition examiantions", Prog. Nucl. Scicence Technol. 2 (2011) p. 5-8.

[40] K. Maeda, "5.24 - Ceramic Fuel-Cladding Interaction", in: Comprehensive Nuclear Materials, R.J.M. Konings, R.E.B.T. Stoller (Eds.), 2nd ed., Elsevier, Oxford, pp. 775-810. 10.1016/B978-008-056033-5.00068-9.

[41] K. Konashi, T. Yato, H. Kaneko, "Radiation effect on partial pressure of fission product iodine", $J$. Nucl. Mater. 116 (1983) p. 86-93. https://doi.org/10.1016/0022-3115(83)90296-9. 